\title{
Molecular Adaptations of Mammalian Hypoxia Tolerance: Regulation of Oxidative Damage, Neuroprotection, and MicroRNA
}

\author{
Hanane Hadj-Moussa
}

B.Sc. Hon. Carleton University, 2015

M.Sc. Carleton University, 2017

A thesis submitted to the Faculty of Graduate Studies and Research in partial fulfilment of the requirements for the degree of

Doctor of Philosophy

Department of Biology

Carleton University, Ottawa, Ontario, Canada

(C) Copyright 2021

Hanane Hadj-Moussa 


\section{Abstract}

Prolonged exposure to limited oxygen can be lethal. Investigating the biological consequences of oxygen-deprivation in a hypoxia tolerant mammalian model can provide us with novel insights that could be applied to alleviate the ischemic insults experienced during stroke, or to better tolerate the hypoxia of high-altitude. Naked mole-rats (Heterocephalus glaber) represent nature's solution to the problem of both acute and chronic oxygen limitation among mammals, solutions that have developed over evolutionary time. In this thesis I investigate their unique adaptations. The data I collected paints a picture of intricate signalling mechanisms in place to facilitate metabolic reorganization and protection during hypoxia. I determine that naked mole-rats are not as vulnerable to hypoxia-induced oxidative damage, as compared to hypoxia intolerant animals, and that brains appear to be the most resilient. The cell-survival proteins I profile implicate the induction of mechanisms responsible for conserving energy and maintaining neural integrity under low oxygen levels. Next, I perform the first microRNA-sequencing analysis in naked mole-rats, focusing on the hypoxic brain. Hypoxia-induced microRNAs suppress ATP-expensive processes, activate central signalling pathways, and coordinate a shift to non-fructose based anaerobic glycolysis. I then examine global metabolic reorganization and characterize a microRNAmediated, AMPK-driven shift to carbohydrate metabolism in hypoxic skeletal muscles that may support tissue-specific prioritization of energy for more essential organs. Taken together, these findings advance our understanding of mammalian hypoxia tolerance and highlight the molecular mechanisms and complex layered regulatory controls required to endure frequent hypoxia exposures, as well as provide directions for future studies. 


\section{Statement of contribution}

This integrated $\mathrm{PhD}$ thesis is composed of four main research papers. Three of which have been published in peer-reviewed academic journals, and a fourth that is awaiting journal submission. Except where specified, I designed and performed all experiments, analyzed the data, performed all bioinformatic analyses, created figures, and wrote all chapters. Dr. Kenneth B. Storey supported all chapters through funding, guided this research program, performed editorial review, and final approval of all chapters. Janet M. Storey provided editorial review.

\section{Chapter 2:}

This study is awaiting submission to a peer-reviewed journal:

Hadj-Moussa H, Eaton L, Cheng H, Pamenter ME, \& Storey KB. Naked mole-rats resist the accumulation of hypoxia-induced oxidative damage.

\section{Chapter 3:}

This study is published in the Journal of Experimental Zoology Part A: Ecological and Integrative Physiology:

Hawkins LJ*, Hadj-Moussa H*, Nguyen VC, Pamenter ME, \& Storey KB. (2019). Naked molerats activate neuroprotective proteins during hypoxia. Journal of Experimental Zoology Part A: Ecological and Integrative Physiology, 331(10), 571-576.

Liam J. Hawkins assisted with the protein assays and manuscript writing. Vu Chau Nguyen and Matthew E. Pamenter performed the animal experiments. The publisher, Wiley, allows for inclusion of this article, in part or in full, in a thesis or dissertation as part of the rights of the author for personal use. 


\section{Chapter 4:}

This study is published in the Journal of Cellular Physiology:

Hadj-Moussa H, Pamenter ME, \& Storey KB. (2021). Hypoxic naked mole-rat brains use microRNA to coordinate hypometabolic fuels and neuroprotective defenses. Journal of Cellular Physiology, 236(7), 5080-5097.

Matthew E. Pamenter performed the animal experiments. The publisher, Wiley, allows for inclusion of this article, in part or in full, in a thesis or dissertation as part of the rights of the author for personal use.

\section{Chapter 5:}

This study is published in the Journal of Experimental Biology:

Hadj-Moussa H, Chiasson S, Cheng H, Eaton L, Storey KB, \& Pamenter ME. (2021) MicroRNAmediated inhibition of AMPK coordinates tissue-specific downregulation of skeletal muscle metabolism in hypoxic naked mole-rats. Journal of Experimental Biology. 1;224(15): jeb242968.

Sarah Chiasson performed the whole animal respirometry experiments. Hang Cheng and Liam Eaton performed in vivo hypoxia experiments for tissue collection, and dissections. Matthew E. Pamenter contributed reagents and analysis. The publisher, The company of Biologists, allows for inclusion of this article, in part or in full, in a thesis or dissertation as part of the rights of the author for personal use. 


\section{Acknowledgements}

My first thanks go to my supervisor Dr. Kenneth Storey. I am grateful for all your support, both life and science advice, and for your encouraging words interspersed with your hilarious comebacks and tough love. Your completely random stories and unlimited supply of caffeine in all shapes and forms are what have kept me going all these years. I appreciate how you threw me into the deep end of 'crocodile-infested' waters whenever the opportunity presented itself and for occasionally fishing me back out. I would also like to thank Jan Storey for everything she has done to make me into the scientist I am today. Thanks for helping me with my writing and for always being available for a chat. I will miss you both and I look forward to annoying you in the future.

I want to thank everyone that I have worked with in the Storey lab. Special thanks go to Liam and all the crazy ideas we explored together, it was a blast! Thanks for keeping me in line. Rasha and Ranim, thanks for all the fun times, the gossip, the food, the workouts, and the constant jokes. Kama and Christie, thanks for being down for a coffee break at a moment's notice, you guys are great conversation and great friends. Bryan, Sanoji, and Alex, thanks for keeping things interesting with the breakfasts and hikes. Sam and Stuart, you two have been with me since undergrad, I'm happy that we all pulled through! And a special thanks goes to Saumya, you have been the best of friends and have always been there to keep it light, vent, and most importantly, entertain!

My biggest thanks goes to my parents for raising me to be the nerd that I am today. You have both been incredibly supportive, I love you and cannot thank you enough for everything that you have done for me. I also need to thank my siblings. I say need because these acknowledgements would be incomplete without them, and because I have been peer-pressured into adding them all in... Ayoub, you have always been my biggest of brothers and bosses, I hope I can one day be as successful as you. Dalal, thanks for all your support, for always being there, the late-night pick ups, and delicious foods. Nabil, my brether, thanks for continually asking me if I found the cure. Every. Single. Day... Firdous, you are my partner in crime, thanks for constantly taking advantage of how gullible I am, we both know “I'm not an idiot!”. Feyrouz and Afou, I love you guys. I also want to thanks my little monkeys, Hakim, Hamza, and Maher, you guys are hilarious. Hakim, I hope you grow up to be the great scientist I know you are, go change the world my little dude! And my last thanks go to Bachir, I am looking forward to all the good times we will have. 


\section{Table of contents}

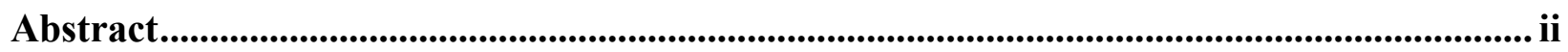

Statement of contribution............................................................................................................ iii

Acknowledgements .................................................................................................................... v

Table of contents .......................................................................................................................... vi

List of abbreviations .................................................................................................................

List of figures................................................................................................................................... $\mathrm{xV}$

List of appendices............................................................................................................................... $\mathrm{xx}$

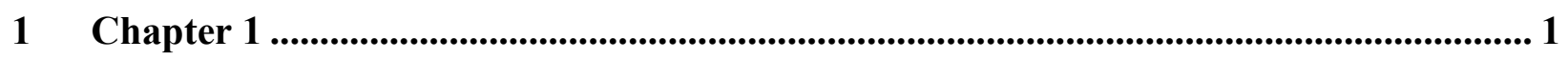

$1.1 \quad$ Naked mole-rats, unusual champions of hypoxia tolerance............................................ 2

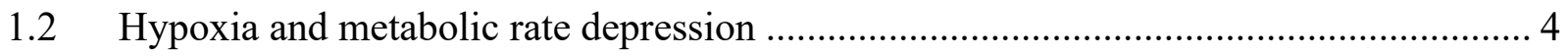

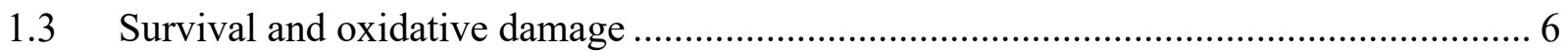

1.4 MicroRNA gene silencing during metabolic rate depression ...................................... 8

$1.5 \quad$ Hypothesis and discovery-driven objectives ............................................................. 10

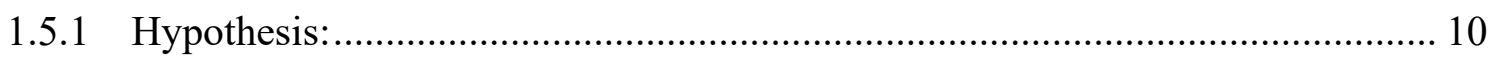

1.5.2 Objective 1: Multi-tissue profiling of oxidative damage markers and neutralization repair mechanisms in hypoxic H. glaber .................................................. 10

1.5.3 Objective 2: Identify the neuronal pro-survival mechanisms in place to mitigate

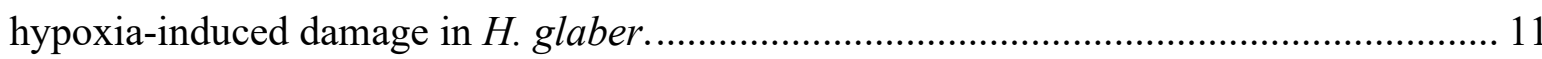

1.5.4 Objective 3: Regulation of microRNA-mediated neuroprotective and fuel switching in hypoxic $H$. glaber brains.

1.5.5 Objective 4: Explore AMPK-driven metabolic reorganization and reprioritization in hypoxic H. glaber skeletal muscles. ........................................................................ 12

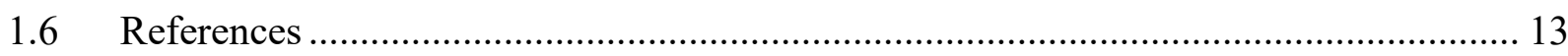

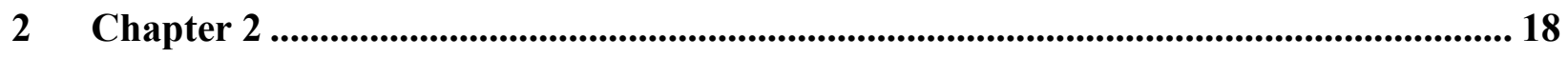

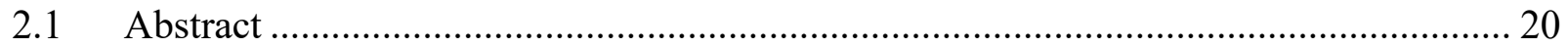

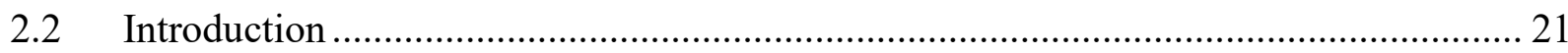

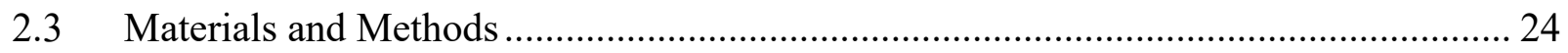

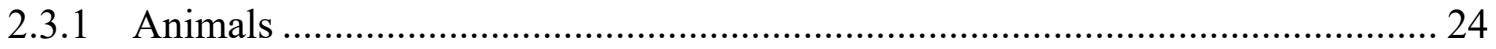




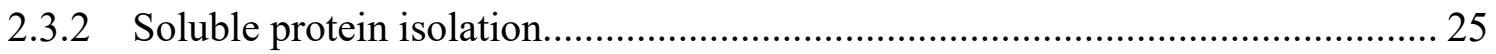

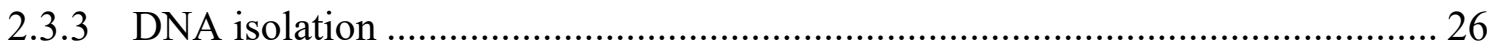

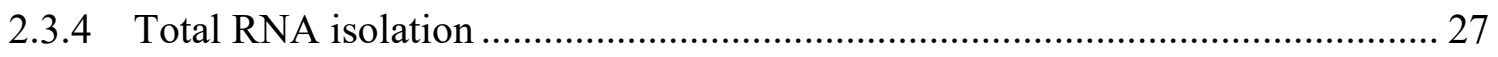

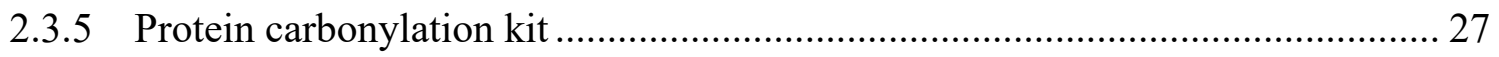

2.3.6 DNA and RNA oxidative damage assays ....................................................... 28

2.3.7 Thiobarbituric Acid Reactive Substances (TBARS) assay ……………….......... 29

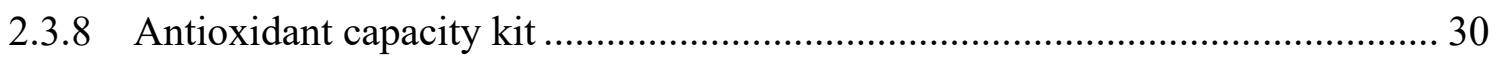

2.3.9 Relative abundance of DNA damage repair proteins......................................... 31

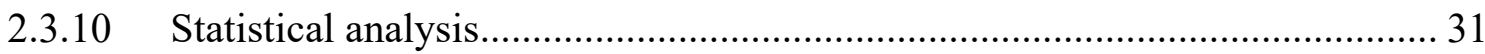

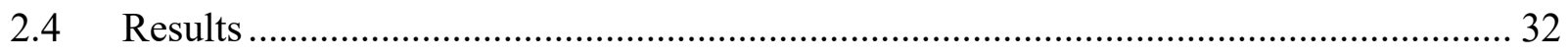

2.4.1 Hypoxia has no effect on protein carbonylation levels ....................................... 32

2.4.2 DNA oxidative damage is minimal during hypoxia........................................... 32

2.4.3 RNA oxidative damage only observed in kidney ............................................. 32

2.4.4 All tissues except brain undergo lipid peroxidation during hypoxia .................. 33

2.4.5 Brains and hearts display the most antioxidant defense capacity ……................ 33

2.4.6 Dynamic tissue-specific regulation of DNA damage repair during hypoxia...... 34

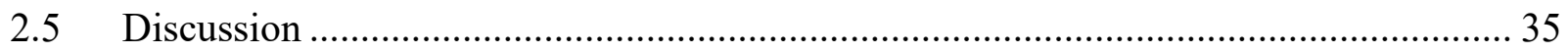

2.5.1 No protein, little nucleic acid, and increased lipid oxidative damage during hypoxia 36

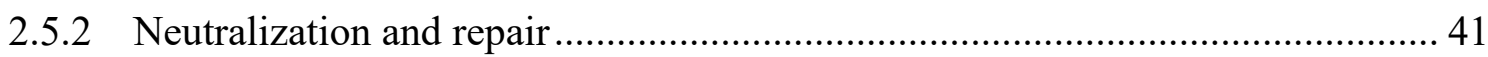

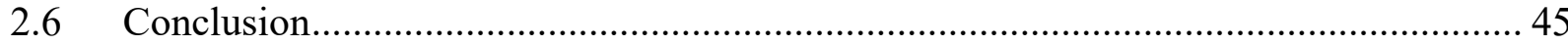

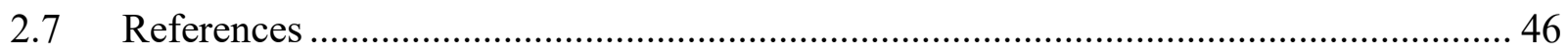

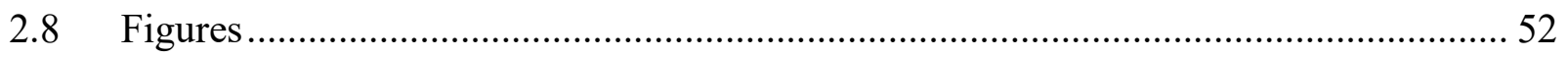

3 Chapter 3 ............................................................................................................................. 58

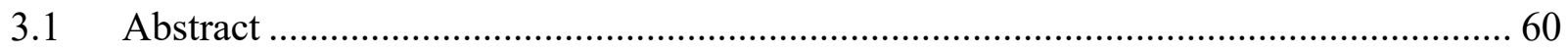

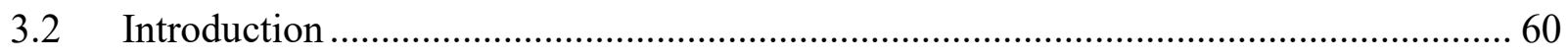

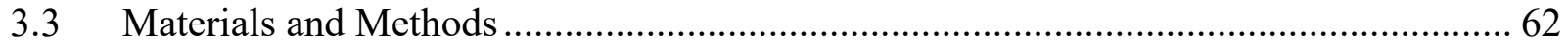

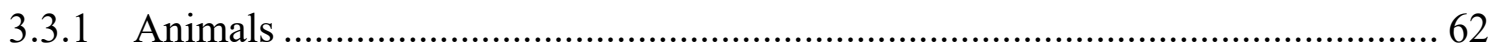

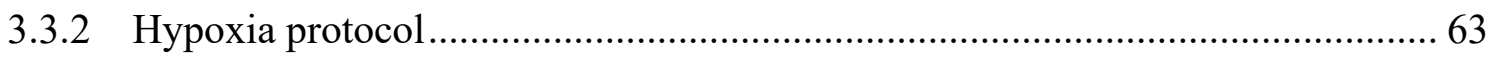

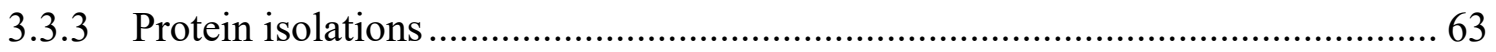

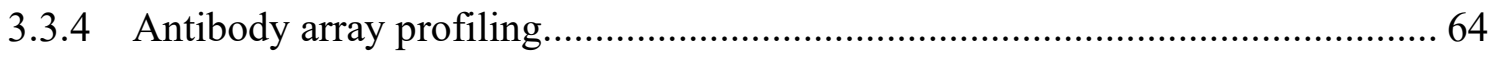




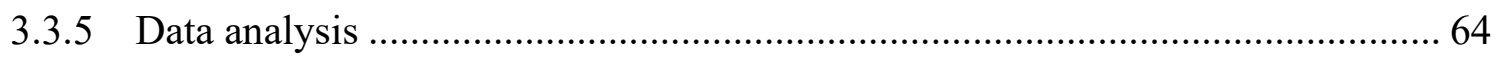

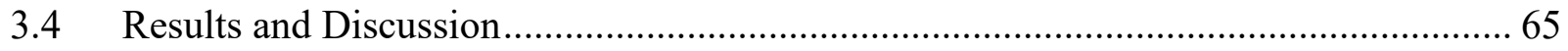

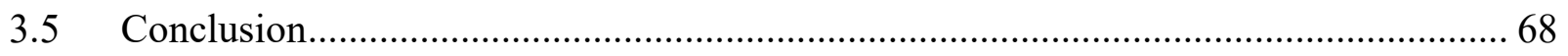

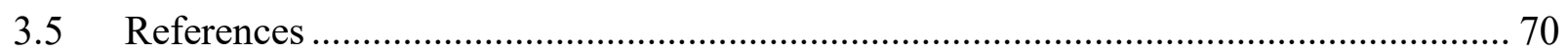

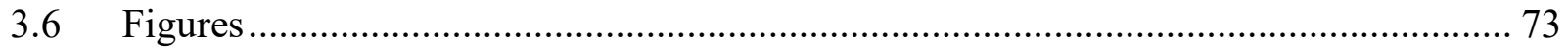

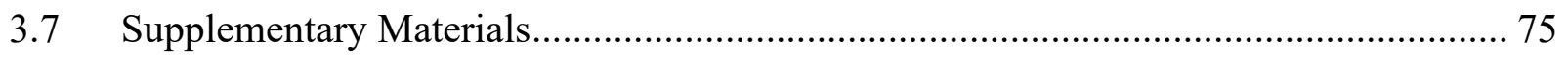

4 Chapter 4 ......................................................................................................................................... 76

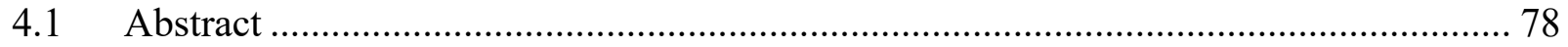

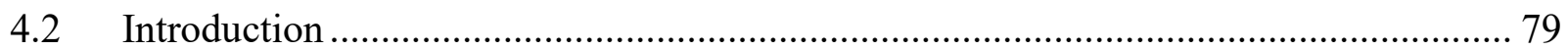

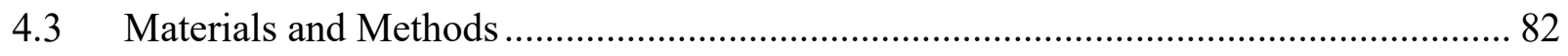

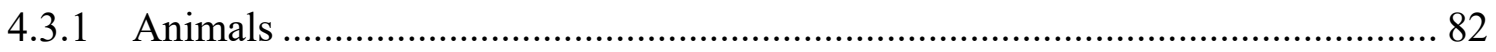

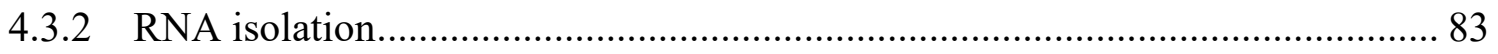

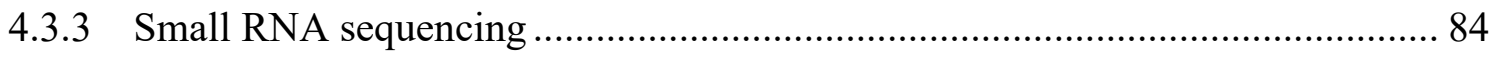

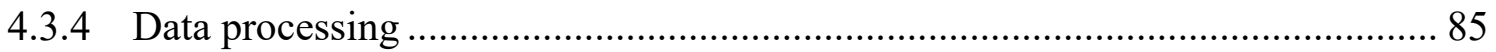

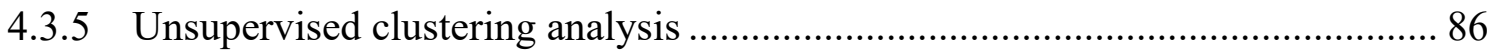

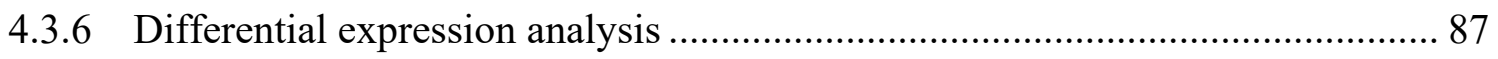

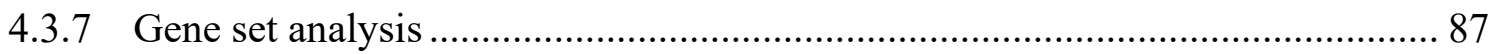

4.3.8 RNA polyadenylation and cDNA synthesis ..................................................... 88

4.3.9 RT-qPCR amplification of miRNA and mRNA ……………........................... 89

4.3.10 Quantification of RT-qPCR and statistics ..................................................... 90

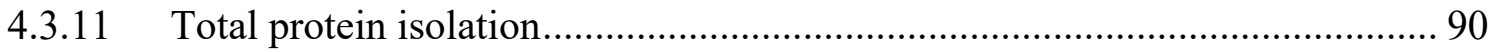

4.3.12 Western blotting, protein quantification, and analysis ................................. 91

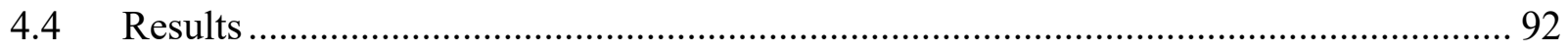

4.4.1 Small RNA-seq analysis and identification of conserved miRNAs.................... 92

4.4.2 MiRNA differential expression analysis .......................................................... 92

4.4.3 RT-qPCR validation of RNA-seq analysis........................................................ 93

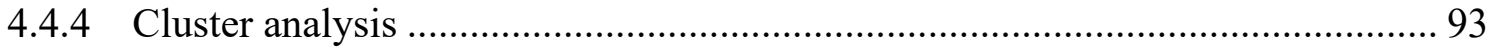

4.4.5 Gene set analysis ......................................................................................... 94

4.4.6 Transcript mRNA levels of downstream targets ................................................ 95

4.4.7 Protein expression levels of downstream miRNA targets.................................... 96

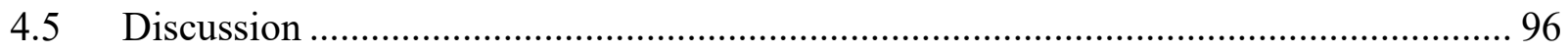


4.5.1 Overall miRNA trends during hypoxia ..................................................... 96

4.5.2 Hypoxia-mediated miRNA inhibition of non-essential energy expensive processes 97

4.5.3 Switching fuel sources during hypoxia .................................................. 99

4.5.4 Neuroprotection or neuro-vulnerability?................................................... 103

4.5.5 Signal transduction regulated by hypoxia-induced miRNAs ......................... 105

4.5.6 A comparison of hypoxia-responsive miRNAs .......................................... 107

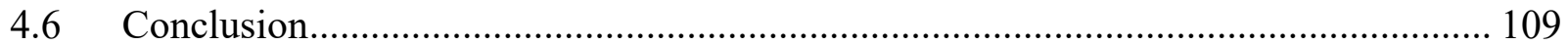

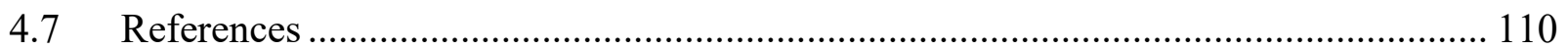

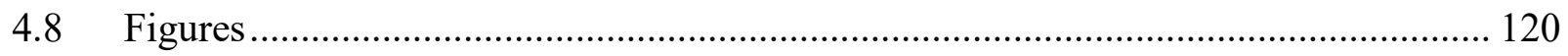

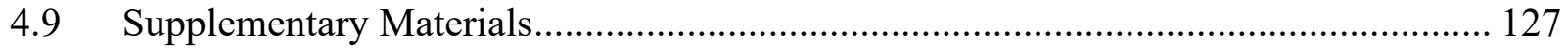

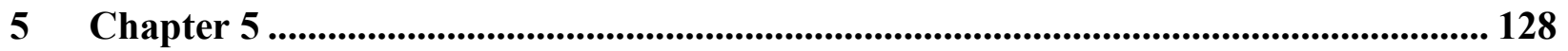

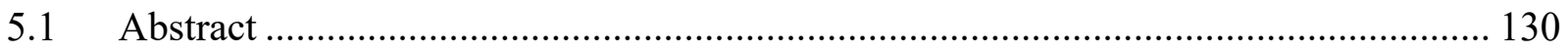

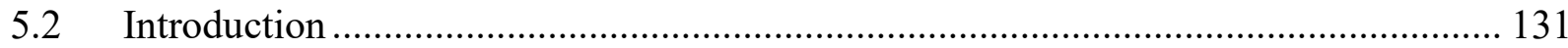

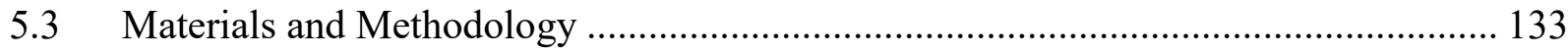

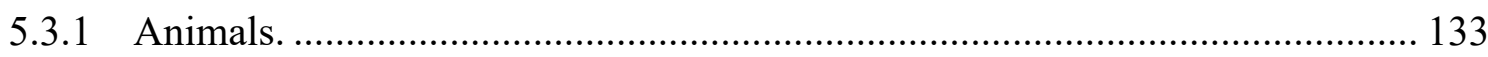

5.3.2 Whole Animal Respirometry. .......................................................... 133

5.3.3 Experimental Design for Tissue Collection. ................................................ 135

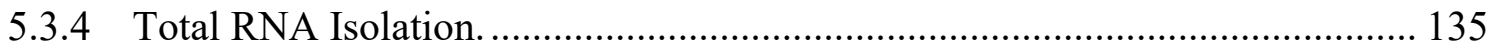

5.3.5 MiRNA Quantification and Data Analysis. ............................................. 136

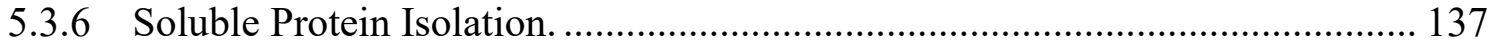

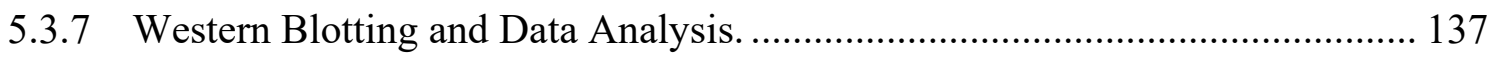

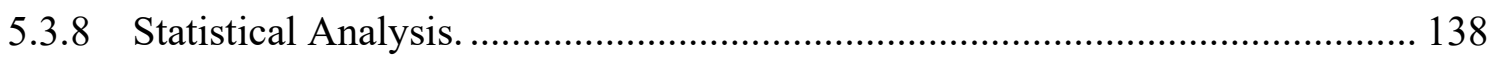

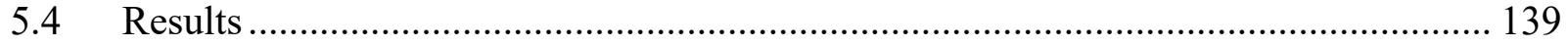

5.4.1 Naked mole-rats exhibit robust metabolic rate suppression and a fuel switch from mixed lipids to carbohydrates in acute hypoxia......................................................... 139

5.4.2 AMPK enzyme subunit expression and phosphorylation are altered during acute hypoxia. 139

5.4.3 Carbohydrate and lipid catabolic enzymes are reduced during hypoxia........... 140

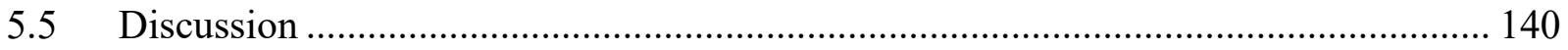


5.5.1 Hypoxia downregulates AMPK and downstream regulators of carbohydrate metabolism.

5.5.2 AMPK-associated miRNAs are hypoxia-responsive in skeletal muscle. ......... 143

5.5.3 A mechanism for tissue-specific energy prioritization in hypoxia?.................. 144

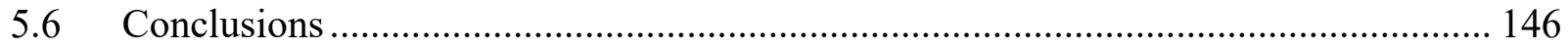

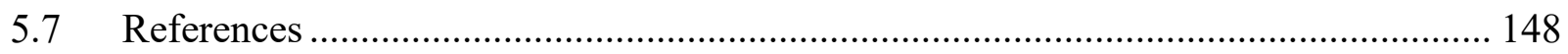

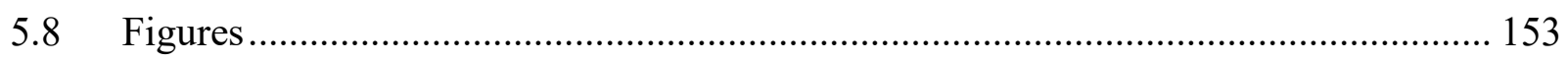

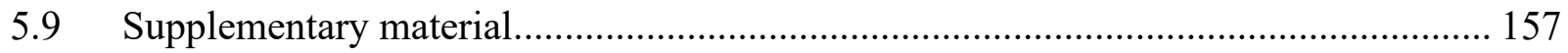

6 Chapter 6 ..................................................................................................................... 162

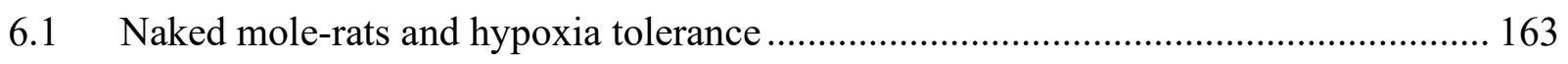

6.2 Minimal oxidative damage and enhanced protection during hypoxia ........................ 164

6.3 MicroRNA drives fuel conservation and reprioritization during hypoxia ................... 166

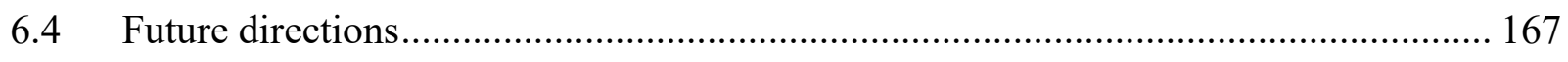

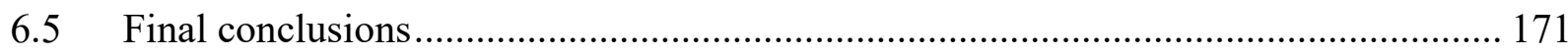

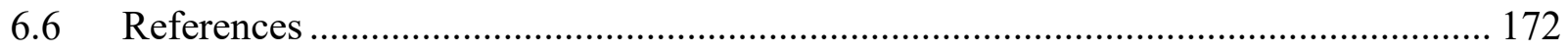

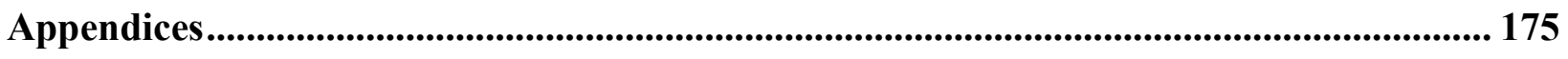

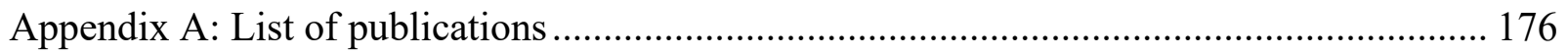

Appendix B: Communications at scientific meetings ....................................................... 180

Appendix C: Representative western blot images............................................................. 183

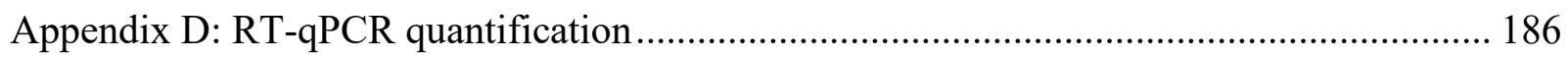

Appendix E: RT-qPCR primers used for mRNA and microRNA analysis ............................ 189 


\section{List of abbreviations}

8-OHG 8-hydroxyguanosine

8-OHdG 8-oxo-2'deoxyguanosine

ABTS 2,2'-azino-di-(3-ethylbenzthiazoline sulfonate)

ACC1 Acetyl-CoA carboxylase

ADAMTS1 Thrombospondin motifs 1

Akt Protein kinase B

AMP Adenosine Monophosphate

AMPK 5'-AMP-activated protein kinase

ANOVA Analysis of variance

APRIL A proliferating-inducing ligand

ATM ATM serine/threonine kinase

ATP Adenosine triphosphate

ATR Ataxia telangiectasia and $\operatorname{Rad} 3$ related protein

BCL2 B-cell CLL/lymphoma 2

BP Biological Processes

CA9 Carbonic anhydrase 9

CC Cellular component

CDK Cyclin-dependent kinase

CITED2 CBP/P300-interacting transactivator 2

Chk1 Checkpoint kinase 1

Chk2 Checkpoint kinase 2

COX2 Cytochrome c oxidase 2

Cq Cycle threshold

CREB cAMP response element-binding protein

CYC Cytochrome c

DE Differential expression

DKK4 Dickkopf WNT signaling pathway inhibitor 4

DNA Deoxyribonucleic acid

DNPH 2,4-dinitrophenylhydrazine 


\begin{tabular}{|c|c|}
\hline dsRNA & Double stranded RNA \\
\hline eBayes & Empirical Bayseian \\
\hline ELISA & Enzyme-linked immunosorbent assay \\
\hline FABP1 & Fatty-acid binding protein 1 \\
\hline FOXA1 & Forkhead Box A1 \\
\hline FOXO3A & Forkhead box O3 \\
\hline FOXP2 & Forkhead box protein $\mathrm{P} 2$ \\
\hline GAPDH & Glyceraldehyde-3-phosphate dehydrogenase \\
\hline GLUT & Glucose transporters \\
\hline GO & Gene Ontology \\
\hline GS & Gene set analysis \\
\hline GS & Glycogen synthase \\
\hline GSH & Glutathione \\
\hline GSSG & Glutathione disulfide \\
\hline H2A.X & Histone 2A.X \\
\hline HIF & Hypoxia-inducible factor \\
\hline HK2 & Hexokinase 2 \\
\hline HRP & Horse radish peroxidase \\
\hline HSP & Heat shock protein \\
\hline IDO & Indoleamine 2,3-dioxygenase \\
\hline JNK & JUN N-terminal kinase \\
\hline KEGG & Kyoto Encyclopdia of Genes and Genomes \\
\hline KHK & Ketohexokinase \\
\hline LDH & Lactate dehydrogenase \\
\hline МАPK & Mitogen-activated protein kinase \\
\hline MDA & Malondialdehyde \\
\hline MDM2 & Mouse double minute 2 homolog \\
\hline MIQE & Minimum information for publication of quantitative real-time PCR \\
\hline miRNA/ miR & MicroRNA \\
\hline MF & Molecular Function \\
\hline mRNA & Messenger RNA \\
\hline
\end{tabular}




\begin{tabular}{|c|c|}
\hline mTOR & Mechanistic target of rapamycin \\
\hline NFкB & Nuclear factor kappa-light-chain-enhancer of activated B cells \\
\hline Nrf2 & Nuclear factor erythroid 2-related factor 2 \\
\hline OxymiR & Oxygen responsive microRNAs \\
\hline PAX6 & Paired box protein Pax- 6 \\
\hline $\mathbf{P C}$ & Principal component \\
\hline PCA & Principal component analysis \\
\hline p21/CIP1 & CDK-interacting protein 1 \\
\hline p27/Kip1 & Cyclin-dependent kinase inhibitor 1B \\
\hline P-bodies & Processing-bodies \\
\hline PI3K & Phosphatidylinositol-3-kinase-like \\
\hline PON & Paraoxonase \\
\hline PUFA & Polyunsaturated fatty acids \\
\hline RER & Respiratory exchange ratio \\
\hline RISC & RNA-induced silencing complex \\
\hline RNA & Ribonucleic acid \\
\hline RNAi & RNA interference \\
\hline RNS & Reactive nitrogen species \\
\hline ROS & Reactive oxygen species \\
\hline RT-qPCR & Reverse transcription quantitative Polymerase chain reaction \\
\hline SEM & Standard error of the mean \\
\hline SIRT2 & Sirtuin 2 \\
\hline SDS-PAGE & Sodium Dodecyl Sulphate-Polyacrylamide Gel Electrophoresis \\
\hline Seq & Sequencing \\
\hline Snord & Small nucleolar RNA \\
\hline SOD & Superoxide dismutase \\
\hline Sp1 & Specificity protein 1 \\
\hline SREBP2/ SREBF2 & Sterol regulatory element binding transcription factor 2 \\
\hline STAT3 & Signal transducer and activator of transcription 3 \\
\hline STPD & Standard temperature, pressure, dry \\
\hline TBA & Thiobarbituric acid \\
\hline
\end{tabular}


TBARS

TBST

TCA

TGF $\beta$

TIGAR

TRIM27

Trolox

tRNA

TXN

ULK1

UTR

$\dot{\mathrm{V}} \mathrm{CO}_{2}$

VHL

$\dot{\mathrm{VO}}_{2}$

WAT

YY1
Thiobarbituric Acid Reactive Substances

Tris-Buffered Saline and Tween 20

Trichloroacetic acid

Transforming growth factor- $\beta$

TP53-inducible glycolysis and apoptosis regulator

Tripartite motif 27 protein

6-hydroxy-2,5,7,8-tetramethylchoman-2-carboxylic acid

Transfer RNA

Thioredoxin-1

Unc-51 like autophagy activating kinase

Untranslated region

Carbon dioxide production

Von Hippel-Lindau tumor suppressor

Oxygen consumption

White adipose tissue

Yin yang1 


\section{List of figures}

Figure 1.1. Consequences of microRNA targeting for gene and protein expression. In red, perfect miRNA complementarity to target mRNA transcripts induces permanent mRNA degradation. In yellow, imperfect miRNA complementarity with mRNA transcripts temporarily suppresses translation and directs mRNA transcripts into temporary storage in stress granules and processing bodies. In green, the reduced expression of miRNAs during stress conditions can act to promote the translation of mRNAs that encode protective, prosurvival proteins, by removing miRNA-induced inhibition (adapted from Hadj-Moussa et al., 2021).

Figure 2.1. Protein carbonylation levels in hypoxic naked mole-rat tissues. Histogram comparing relative protein carbonylation $(\mathrm{nmol} / \mathrm{mL})$ in normoxic (control), $4 \mathrm{~h}$ hypoxic, and $24 \mathrm{~h}$ hypoxic naked mole-rat tissues (brain, heart, liver, skeletal muscle, kidney, WAT). Data are means $\pm \operatorname{SEM}(n=4$ independent biological replicates from different animals), with normoxic mean values standardized to 1 . Statistical analysis was preformed for each tissue using a one-way ANOVA with a Dunnett's post-hoc test $(p \leq 0.05)$ and significant changes are indicated with an asterisk. 52

Figure 2.2. DNA oxidative damage levels in hypoxic naked mole-rat tissues. Histogram comparing relative DNA oxidative damage levels of $4 \mathrm{~h}$ hypoxic and $24 \mathrm{~h}$ hypoxic exposures, relative to normoxic (control) in six naked mole-rat tissues (brain, heart, liver, muscle, kidney, WAT). Other information as per Figure 2.1.... 53

Figure 2.3. Relative RNA oxidative damage in hypoxic naked mole-rat tissues. Histogram comparing relative RNA oxidative damage levels of $4 \mathrm{~h}$ hypoxic and $24 \mathrm{~h}$ hypoxic exposures, relative to normoxic (control) in six naked mole-rat tissues (brain, heart, liver, muscle, kidney, WAT). Other information as per Figure 2.1. 54

Figure 2.4. Lipid peroxidation as measured by TBARS concentrations in hypoxic naked mole-rat tissues. Histogram comparing relative TBARS concentration $(\mu \mathrm{M})$ of normoxic (control), $4 \mathrm{~h}$ hypoxic, and $24 \mathrm{~h}$ hypoxic naked mole-rat tissues (brain, liver, muscle, kidney, WAT). Other information as per Figure 2.1....................................................... 55 
Figure 2.5. Total antioxidant capacity in hypoxic naked mole-rat tissues. Histogram comparing relative antioxidant capacity of normoxic (control), $4 \mathrm{~h}$ hypoxic, and $24 \mathrm{~h}$ hypoxic naked mole-rat tissues (brain, heart, liver, muscle, kidney, WAT). Other information as per

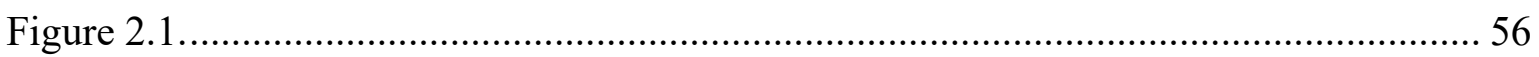

Figure 2.6. Regulation of DNA damage and repair proteins in hypoxic naked mole-rat tissues. Histograms compare relative protein levels of phosphorylated Chk1 (Ser345), Chk2 (Thr68), H2A.X (Ser139) and p53 (Ser15), as well as total protein levels of ATR, MDM2 and p21. (A) Brain, (B) Heart, (C) Liver, (D) Skeletal muscle, (E) Kidney, and (F) WAT. Other information as per Figure 2.1.

Figure 3.1. Summary of the central pro-survival protein changes observed in hypoxic naked mole-rat brains.

Figure 3.2. Cell-stress related protein levels in response to hypoxia in the brain of naked mole-rats. [A] Overview of measured protein level changes. Of the proteins measured, 42\% $(11 / 26)$ were upregulated in the hypoxic condition, $46 \%(12 / 26)$ did not change statistically, and 12\% (3/26) proteins were downregulated. [B] Hypoxia to normoxia protein level ratios for 26 cell-stress related proteins. Data are mean $\pm \mathrm{SEM}$ * indicates statistically significant differences between normoxic and hypoxic animals $(p<0.05)$ as determined by Student's $t$ test with FDR correction. Eight animals were used in total, four in the normoxic group and 4 in the hypoxic group. 74

Figure 4.1. Differential expression analysis. Volcano plot for DE results, with $p$-value and fold changes. Log2 transformed fold change and $\log 10 p$-values are plotted. Blue represents statistically significant upregulation greater than 1.5-fold. Orange represents statistically significant downregulations greater than 1.5-fold. Top 18 (based on FDR-corrected $p$-values $<0.05)$ differentially expressed miRNAs are marked. 120 
Figure 4.2. RT-qPCR validation of a representative set of significantly differentially expressed miRNAs. Four miRNAs identified to be significantly expressed in miRNA-Seq analysis (miR-132-3p, miR-184-3p, miR-383-5p, and miR-301a-3p) were validate with RTqPCR. Data were analyzed as means of $n=3-4 \pm$ SEM using a Student's t-test, ${ }^{*} p<0.05$.

Figure 4.3. Unsupervised clustering analysis of miRNA expression levels. (A) Heatmap for hierarchical clustering, dendrograms for miRNAs and samples. Light and dark blue bars represent the two clusters that samples were divided into based on their global miRNA expression patterns. (B) Principal component analysis (PCA) on PC1, PC2, and PC3, with density plots on the diagonal.

Figure 4.4. Volcano plot for logistic regression based KEGG and GO term gene set enrichment. Blue dots represent significantly enriched gene sets that lack miRNA inhibition during hypoxia based on the miRNA expression profiles. Orange dots represent significantly enriched gene sets that are miRNA-inhibited during hypoxia. Based on FDR corrected $p$ values $<0.05$ and fold change $>1.5$. (A) KEGG Pathway enrichment, (B) GO Term Molecular Function enrichment, (C) GO Term Cellular Component enrichment, and (D) GO Term Biological Process enrichment. The complete results for KEGG and GO GS analysis can be viewed in (Supp. Table S4-S7 found online). 123

Figure 4.5. Enriched GO Biological Process semantic network in hypoxic naked mole-rat brains. The 200 significantly enriched GO Biological Processes were summarized by REVIGO and the resulting sematic relation network of summary terms was visualized using Cytoscape. (A) The first network was related to RNA metabolism, external responses to stress stimuli, and tissue development. (B) The second largest network was related to signal transduction and transcription factor pathways, neuronal processes, and regulation of posttranscriptional silencing and post-translational modification contained the most sematic relationships. (C) The third network was related to small ion and amino acid transport and localization. (D) The fourth network comprised of only 3 members was related to ribosomal biogenesis and histone ubiquitination, (E) and the fifth network, also with 3 members was related to lipid biosynthesis and remodelling. Node colour represents FDR corrected $p$ - 
values, node size is proportional to the number of genes each term contains, and grey edges represent sematic relationships. The complete results for GO Biological Process analysis can be viewed in (Supp. Table S7 found online).

Figure 4.6. Transcript mRNA and protein levels of miRNA-downstream metabolic glycolytic targets. (A) Relative mRNA abundance levels of glut $5, k h k$, ldh, and srebp2. Data were analyzed as means of $n=3-4 \pm$ SEM using a Student's t-test, ${ }^{*} p<0.05$, relative to normalized normoxic values. (B) Protein levels of GLUT5, KHK, LDH, and SREBP2. Data were analyzed as means of $n=3-4 \pm$ SEM using a Student's t-test, $* p<0.05$, relative to normalized normoxic values. (C) Schematic of hypoxic miRNA targeting on KHK, LDH, SREBP2, and GLUT5. 125

Figure 4.7. Schematic of miRNA-mediated targeting of fructose and glucose anaerobic glycolysis pathways. Blue miRNAs are upregulated during hypoxia while orange miRNAs are downregulated in hypoxic naked mole-rat brains.

Figure 5.1. Naked mole-rats exhibit metabolic rate suppression and a hypoxic fuel shift from lipids to carbohydrates in acute hypoxia. Summaries of oxygen consumption rate $\left(\mathbf{V}_{\mathrm{O} 2}\right.$; A), carbon dioxide production rate $\left(\mathbf{V}_{\mathrm{CO} 2} ; \mathrm{B}\right)$, and respiratory exchange ratios (RER; $\left.\mathbf{V}_{\mathrm{CO} 2} / \mathbf{V}_{\mathrm{O} 2} ; \mathrm{C}\right)$ from 6 naked mole-rats exposed to $1 \mathrm{hr}$ of normoxia $\left(21 \% \mathrm{O}_{2}\right.$; white circles) and then 4 hrs of hypoxia ( $3 \% \mathrm{O}_{2}$; black squares). Data are presented as mean $\pm \mathrm{SEM}$. Significant differences are indicated by different letters. $p<0.05$, 1-way ANOVA with Tukey's post-test.

Figure 5.2. 5'-AMP-Kinase (AMPK) function is downregulated in skeletal muscle after acute hypoxia. Relative AMPK protein expression levels in normoxic and hypoxia naked molerat temporalis muscle. (A) Summary of AMPK subunits $(\alpha 1, \alpha 2, \beta)$ and phosphorylated form of p-AMPK (Thr172) protein expression. (B) Images of immunoblot protein expression from (A). Data are presented as mean \pm SEM from $n=5-6$ independent biological replicates for each condition. Significant differences from normoxic controls are indicated by asterisks $\left(^{*}\right)$. $p<0.05$, unpaired student's t-test. 154 
Figure 5.3. Glucose metabolism is downregulated in skeletal muscle after acute hypoxia. Relative protein expression levels in normoxic and hypoxic naked mole-rat temporalis muscle of glucose transporters (GLUT1 and GLUT4), glycogen synthase (GS) and its phosphorylated form p-GS (Ser641/645), and acetyl-CoA carboxylase1 (ACC1) and its phosphorylated form p-ACC1 (Ser79). (A) Summary of protein expression. (B) Images of immunoblot protein expression from (A). Data are presented as mean \pm SEM from $n=6$ independent biological replicates for each condition. Significant differences from normoxic controls are indicated by asterisks $(*) . p<0.05$, unpaired student's t-test. 155

Figure 5.4. 5'-AMP-Kinase (AMPK)-regulating miRNAs are altered in acute hypoxia. (A) Heatmap of the relative expression levels of 55 AMPK-associated miRNAs in naked molerat temporalis muscle tissue. MiRNA expression was measured in $4 \mathrm{hr}$ hypoxia relative to normoxic conditions using quantitative PCR (qPCR). (B) MiRNA expression of significantly changed hypoxia-induced miRNAs in temporalis muscles of naked mole-rats $(p<0.05)$. MiRNA expression was measured in $4 \mathrm{hrs}$ hypoxia relative to normoxic conditions using quantitative PCR (qPCR). Data are means \pm SEM from $n=$ of $3-4$ independent biological replicates for each condition. Significant differences from normoxic controls are indicated by asterisks $\left(^{*}\right) . p<0.05$, unpaired student's t-test. Refer to Table S2 for the relative expression $\pm \mathrm{SEM}$ values of all 55 miRNAs. 156 


\section{List of appendices}

Appendix A: List of publications ......................................... 172

Appendix B: Communications at scientific meetings ........................... 176

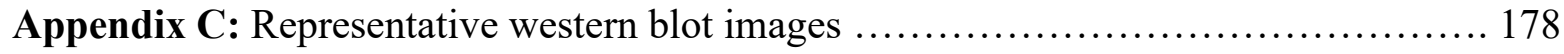

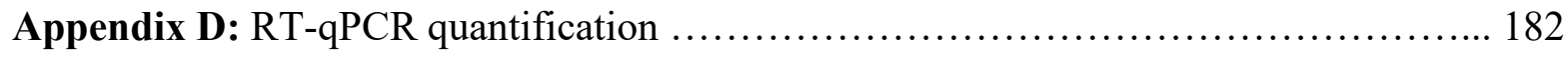

Appendix E: RT-qPCR primers used for mRNA and microRNA analyses .............. 185 
Chapter 1

\section{General Introduction}




\subsection{Naked mole-rats, unusual champions of hypoxia tolerance}

Naked mole-rats (Heterocephalus glaber) are strange creatures. These small rodents display many unique physiological, biochemical, ageing, and behavioural traits that have allowed them to adapt to a subterranean lifestyle. But before discussing their ability to withstand periods of oxygen limitation, the main focus of this thesis, I will highlight a few unique traits that have increased scientific interest in naked mole-rats over recent years (Braude et al., 2021).

Whereas their name implies that they are fully naked, these rodents have tactile hairs distributed all over their body, face, and tail that upon tactile stimulation allow for accurate spatial orientation in their dark, narrow subterranean tunnels (Crish et al., 2003; Jarvis and Sherman, 2002). These tunnels are mainly located underground in East Africa, where these eusocial mammals live in colonies that exhibit distinct reproductive division of labor. Each colony typically has one breeding queen, with the remaining non-breeding animals performing tasks such as pup care, colony defence, and dispersal-like tasks, all of which require specialized adaptations (Mooney et al., 2015; Toor et al., 2020). For example, their extremely poor eyesight is limited to the ability to detect the presence or absence of light (Hetling et al., 2005). As for their auditory system, their hearing appears to have deteriorated and in doing so has shifted to low frequency sounds like other subterranean mammals, making them better suited to the demands imposed by living underground (Braude et al., 2021; Heffner and Heffner, 1993; Okanoya et al., 2018). These adaptations are advantageous for a life underground. Indeed, there are limited observations of naked mole-rats above ground, with their fine, permeable skin also suggesting that they spend minimal time above ground (Brett, 1986; Faulkes and Bennet, 2000).

From a biomedical standpoint, naked mole-rats appear to be immune to typical age-related diseases, but are susceptible to viral and parasitic infections, likely due to their relatively low 
proportion of lymphocytes and lack of natural killer cells (Hilton et al., 2019; Shebzukhov et al., 2019). Curiously, naked mole-rats are insensitive to acid, but their sensory network of neurons respond to capsaicin and histamine (Park et al., 2008). Added to this is the fact that naked molerats are cancer-resistant compared to similar-sized lab mice (Lipman et al., 2004). They have also been shown to be resistant to declines in cognitive function from neurodegenerative accumulation of tau plaques (Edrey et al., 2014, 2013). While it was originally proposed that the naked molerat's exceptional longevity stems from their low experience of oxidative stress and damage, studies have since shown that naked mole-rats accumulate high levels of oxidative damage (Andziak et al., 2006; Andziak and Buffenstein, 2006; De Waal et al., 2013; Mitchell et al., 2007; Pérez et al., 2009). This is offset by cytoprotective mechanisms that have proven a high resiliency to numerous forms of damage, examples of which are discussed below in Section 1.3.

In their underground burrows, these small rodents live together in tunnels that are mainly hypoxic and hypercapnic as compared to the above-ground atmosphere (Holtze et al., 2018). Their tunnel systems are not uniformly occupied, as some areas are always populated (such as nursery chambers) whereas others remain sparsely inhabited. Therefore, as these rodents travel through their burrows, they likely experience highly variable partial pressures of both $\mathrm{O}_{2}$ and $\mathrm{CO}_{2}$ that can lead to intermittent bouts of acute hypoxia and hypercapnia interspersed with normoxic and normocapnic periods. The extent to which naked mole-rats experience hypoxia in their natural environment is still not definitively known, but studies of captive naked mole-rats have demonstrated that these animals can readily survive both acute and chronic hypoxia exposures. Compared to the atmospheric levels of oxygen (21\%) that humans breathe, mole-rats can survive hypoxia exposures at $8 \% \mathrm{O}_{2}$ for days to weeks, as well as several hours at $3 \% \mathrm{O}_{2}$, and up to 18 mins of complete anoxia (Chung et al., 2016; Pamenter et al., 2018; Park et al., 2017). 
To survive in an oxygen-poor environment, naked mole-rats appear to have been shaped by evolutionary pressures to develop numerous physiological and molecular mechanisms that allow them to stay active at oxygen thresholds well below what most mammals can tolerate (Pamenter et al., 2020). For example, their basal metabolic rate is low relative to their body size (McNab, 1979), they have reduced cardiac function (Grimes et al., 2014), and combined with smaller brain volumes (Orr et al., 2016) this allows them to operate while expending less energy when oxygen is limited. During hypoxia, they can further conserve energy by globally reducing metabolic rate and suppressing both thermogenesis (Kirby et al., 2018) and heart rate (Pamenter et al., 2019).

During hypoxia-induced metabolic rate depression mole-rats also suppress energy expensive processes such as de novo protein translation (Al-attar et al., 2020). This is performed alongside a shift to carbohydrate centered metabolism, where for example, anoxic brains were shown to switch to anaerobic glycolysis and fructose catabolism (Park et al., 2017). Additionally, like other animals undergoing global reductions in metabolic rate, naked mole-rats also activate various pro-survival pathways, examples of which are discussed in Section 1.3. While naked mole-rats are equipped with mechanisms that facilitate hypoxia survival, key questions about these adaptations remain unanswered, and this thesis aims to investigate them.

\subsection{Hypoxia and metabolic rate depression}

When animals do not possess the adaptations necessary to overcome environmental stresses that threaten the source of one or more metabolic inputs (e.g., biomolecules, oxygen, water), they quickly deplete their internal energy reserves such that prolonged stress exposure can prove lethal. Yet despite oxygen being the basis for life on Earth, many animals can survive acute and chronic oxygen deprivation, on both short and long timescales (Larson et al., 2014). When oxygen is 
limited, animals react in one of two ways; they can elicit an immediate response to an acute oxygen-limited bout, or can elicit a preparatory response, where they have evolved complex mechanisms to anticipate and endure prolonged hypoxic/anoxic periods (Giraud-Billoud et al., 2019; Larson et al., 2014). Regardless of the response taken, the metabolic consequences and anaerobic end-products resulting from partial or total restriction of oxygen must be dealt with immediately. To do so, hypoxia and anoxia tolerant animals have developed adaptations that allow them to protect and defend against these insults, often by employing a strategy of metabolic rate depression.

Retreating into a hypometabolic state allows animals to establish a new balance between limited resource availability and resource consumption, the transition to a new lower metabolic rate is achieved by suppressing non-vital metabolic pathways. By reducing ATP demand to a level that can be met by less-efficient anaerobic ATP-generating pathways (typically anaerobic glycolysis), many vertebrates (mainly poikilotherms) and invertebrates can deal with hypoxia and/or anoxia for prolonged periods of time (Krivoruchko and Storey, 2015). This requires the coordination of numerous pathways to suppress non-essential processes and reprioritize ATP for vital cellular functions such as transcription and translation of stress-induced proteins, suppressing rates of transmembrane active transport, along with sweeping changes to enzyme/protein posttranslational modifications, among other adjustments (Storey and Storey 1990). This occurs simultaneously with the activation of select pathways that support pro-survival mechanisms (Krivoruchko and Storey, 2010). Such reprogramming has been shown to occur at all cellular regulatory levels, ranging from epigenetic modifications on DNA and histones, modulation of transcription factor activity, post-transcriptional regulation via non-coding RNAs, and differential post-translational modifications, among others (Storey, 2015). 
A common strategy for hypoxia tolerance is metabolic depression, as this allows animals to survive on limited oxygen supplies. Naked mole-rats are no exception. Respiratory exchange ratio (RER) calculations of naked mole-rats indirectly support a whole-animal metabolic fuel switch from lipid-based metabolism during normoxia to more carbohydrate-based metabolism during hypoxia (Pamenter et al., 2019). This requires intricate coordination of many cellular controls, but the roles played by many cellular mechanisms to regulate this metabolic fuel switch are still unknown. In this thesis, I investigated non-coding RNA controls, discussed below in Section 1.4, and investigated the master energy sensor, the 5'-AMP-activated protein kinase (AMPK).

AMPK-driven metabolic fuel reprioritization plays a central role in regulating energy balance when ATP availability is limited. AMPK activates catabolic processes to improve ATP production, while simultaneously inhibiting ATP-expensive anabolic processes. It is activated by changes in the intracellular AMP:ATP ratio, where the breakdown of ATP to AMP favours activation of AMPK, in turn inducing the remodelling of cellular energetics and metabolic fuel usage (Hardie et al., 2012). The post-translational modification of AMPK and its downstream targets involved in fatty acid metabolism, glucose uptake, and glycogen synthesis can give a strong indication of the state of metabolism in a tissue and possible tissue-specific energy prioritization during hypoxia (Kahn et al., 2005; Rider, 2016; Winder et al., 2003). This is one area that was explored in this thesis with respect to naked mole-rat hypoxia tolerance.

\subsection{Survival and oxidative damage}

Genetic, biochemical, and molecular studies indicate an evolutionarily conserved 'biochemical unity' to the underlying mechanisms of stress-induced adaptation to extreme environments. Discussed above is the common theme of metabolic suppression and shifts in fuel 
preference, with the other common strategy being the enhancement of protective measures. This is needed as cycling between normoxic and hypoxic conditions can lead to elevation of reactive oxygen species (ROS) typically experienced during rapid oxygen reperfusion, when bursts of ROS are generated because of increases in respiration and oxygen consumption (Hochachka et al., 1996).

To assess oxidative stress, studies monitor the ROS-induced damage that affects macromolecule structure and function, including peroxidation of fatty acids, modification of protein carbonyls, and the formation of double bond adducts on nucleic acids resulting in 8-oxo2'deoxyguanosine (8-OHdG) on DNA and the formation of 8-hydroxyguanosine (8-OHG) on RNA (Lewis et al., 2013). Curiously, when compared to mice, naked mole-rats have higher levels of irreversible oxidative damage including lipid peroxidation (Andziak and Buffenstein, 2006; Edrey et al., 2014), protein carbonylation (Andziak et al., 2006; Pérez et al., 2009), and oxidatively modified DNA (Andziak et al., 2006). Despite this, naked mole-rat mitochondria have a greater capacity for ROS consumption when compared to mice (Munro et al., 2019). But what is different is that the inherently elevated levels of oxidative damage products that naked mole-rats exhibit remain relatively constant throughout their lives, whereas in other species these levels are typically found to increase as organisms age, and have formed the basis for the oxidative theory of ageing.

Additionally, the cellular responses to oxidative damage can be as important in determining stress resiliency and the detrimental effect of that damage as the damage moieties themselves. Examples of protective measures include cytoprotective proteins induced by action of the nuclear factor erythroid 2-related factor 2 (Nrf2) transcription factor (Lewis et al., 2015, 2010), protein chaperone functions (De Waal et al., 2013; Nguyen et al., 2019; Pérez et al., 2009), and the hypoxia-inducible factor pathway (Hawkins et al., 2019; Kim et al., 2011; Pamenter et al., 2020). 
This is in combination with the upregulation of protectants such as antioxidant molecules and repair pathways. Many of these defenses have been found to be enhanced in naked mole-rats, as compared to mice, but they have yet to be examined with respect to hypoxia exposure in naked mole-rats.

\subsection{MicroRNA gene silencing during metabolic rate depression}

MicroRNAs (miRNAs, miR) are principal post-transcriptional regulators of gene expression. These short non-coding RNAs ( $22 \mathrm{nt})$ are a large group of highly conserved transcripts that can target more than $60 \%$ of protein-coding genes in humans and influence almost every aspect of biological function (Bartel, 2009; Ebert and Sharp, 2012). The biogenesis of miRNA is a multistep process that begins with the synthesis of long imperfect dsRNA hairpins in the nucleus and that ends with a single-stranded mature RNA guide strand in the cytoplasm. This mature miRNA guide strand is then incorporated into the RNA-induced silencing complex (RISC) along with Argonaute endonucleases and various other proteins that facilitate the negative regulation and targeting of mRNA via complementary base-pairing (Bartel, 2009).

Each miRNA species can bind to and influence the translation of multiple mRNA transcripts and each mRNA transcript may be targeted by multiple miRNA species, resulting in a multifactorial combination of responses that fine-tune mRNA fate (Figure 1.1.). MicroRNAs exert their function by binding to the $3^{\prime}$ - untranslated region (UTR) of mRNA transcripts. Partial sequence complementarity results in temporary translational suppression and sequestration of the mRNA transcript into cytoplasmic stress granules and processing-bodies (P-bodies); by contrast, perfect complementarity triggers mRNA degradation by Argonaute endonucleases (Figure 1.1.) (Bartel, 2004; He and Hannon, 2004). This interaction occurs at the 5' seed region (nucleotides 
two to eight) of the miRNA guide strand, directing RISC activity towards target mRNAs with complementary motifs in their 3'UTR (Huang et al., 2011). The importance of miRNA regulation is evident in the conservation that miRNA sequences display between distant vertebrate species, where target mRNA 3'UTR binding motifs were found to be $90-100 \%$ conserved, despite the overall low conservation generally observed in 3'UTR regions (Friedman et al., 2008).

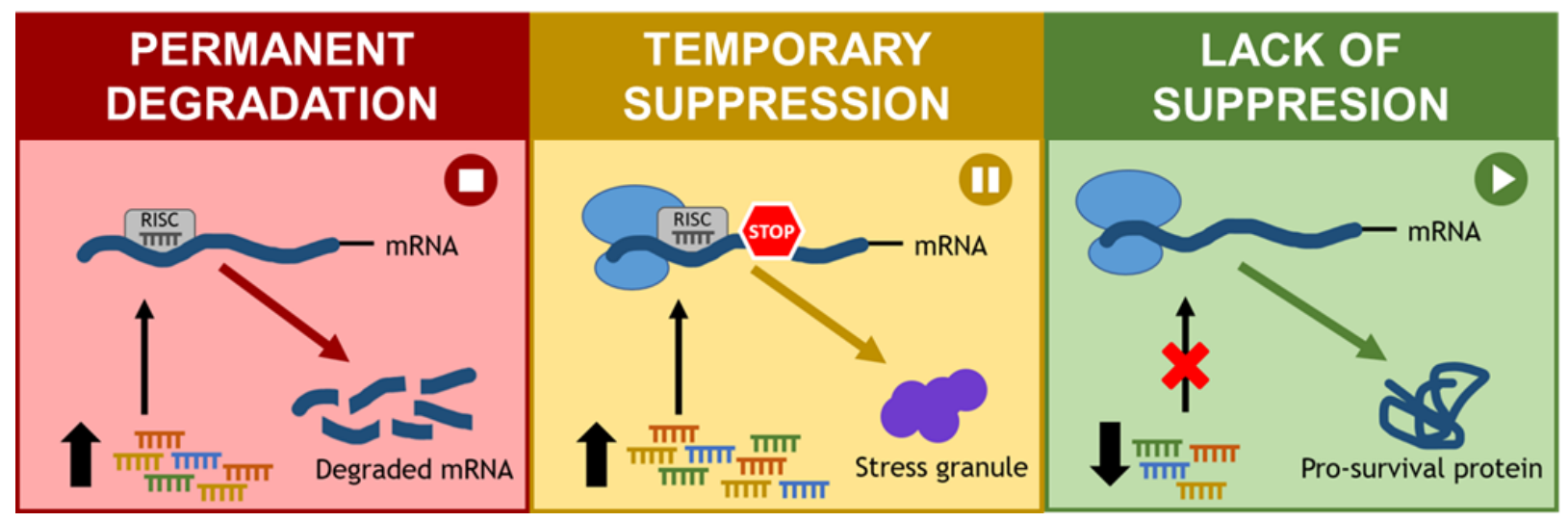

Figure 1.1. Consequences of microRNA targeting for gene and protein expression. In red, perfect miRNA complementarity to target mRNA transcripts induces permanent mRNA degradation. In yellow, imperfect miRNA complementarity with mRNA transcripts temporarily suppresses translation and directs mRNA transcripts into temporary storage in stress granules and processing bodies. In green, the reduced expression of miRNAs during stress conditions can act to promote the translation of mRNAs that encode protective, pro-survival proteins, by removing miRNA-induced inhibition (adapted from Hadj-Moussa et al., 2021).

The importance of miRNAs in facilitating metabolic reprogramming during extreme environmental insults, appears to be centered around five key inherent properties: (1) reversible regulation, (2) rapid targeting, (3) broad control over practically all cellular processes, (4) miRNAs are energetically inexpensive to synthesize and (5) miRNA sequences are highly conserved among different species (Hadj-Moussa and Storey, 2020; Storey, 2015). MicroRNAs have been 
implicated in natural models of hypoxia and anoxia tolerance, with many of these oxygenresponsive miRNAs (OxymiRs) shown to coordinate the metabolic rate depression that allows animals to tolerate reduced oxygen levels. It is interesting to note that when OxymiR responses by evolutionarily disparate animals from diverse environments were compared, that there was a lack of a single unified miRNA response to oxygen limitation, but rather that many of the same processes were being targeted, albeit via different miRNAs, each displaying tissue and stressspecific differential expression patterns (Hadj-Moussa and Storey, 2020).

\subsection{Hypothesis and discovery-driven objectives}

\subsubsection{Hypothesis:}

Molecular responses to hypoxia tolerance in the naked mole-rat (Heterocephalus glaber) require the induction of select master proteins and microRNAs to facilitate pro-survival defenses and global metabolic rate reduction in a temporal and tissue-dependant manner.

This thesis aimed to increase our understanding of hypoxia tolerance in naked mole-rats. This entailed the determination of the state of oxidative damage, the study of pro-survival transcription factors, the involvement of miRNA-mediated mechanisms, and regulation of fuel reprioritization. All of these are outlined in the following objectives:

\subsubsection{Objective 1: Multi-tissue profiling of oxidative damage markers and neutralization} repair mechanisms in hypoxic H. glaber.

As with most hypoxia tolerant animals, naked mole-rats must be able to withstand cycling between normoxic and hypoxic environmental conditions, a feat that brings with it fluctuating 
ROS generation. Enhanced rates of ROS production can lead to the accumulation of irreversible oxidative damage to macromolecules, which can affect the induction of damage neutralization mechanisms. This objective, addressed in Chapter $\mathbf{2}$ of this thesis, is to quantify the DNA, RNA, protein, and lipid oxidative damage accrued during hypoxia, along with the activation of antioxidant and DNA repair mechanisms. The results of these experiments can elucidate whether naked mole-rats are susceptible to oxidative damage and the efficiency of their repair mechanisms during hypoxia.

\subsubsection{Objective 2: Identify the neuronal pro-survival mechanisms in place to mitigate} hypoxia-induced damage in H. glaber.

Brains are highly susceptible to hypoxia-induced damage. Studies in other hypoxia and anoxia tolerant organisms have implicated various transcriptional networks in the coordination of global defenses. Metabolic rate depression is one way to lessen damage and entails the coordination and crosstalk with many of the key signal transduction pathways. This objective, examined in Chapter $\mathbf{3}$ of this thesis, is aimed at investigating how the cell-stress-related protein landscape changes in response to hypoxia and, more specifically, how the regulation of transcriptional networks in hypoxic brains can provide a snapshot of the cell stress factors in place to mitigate damage.

\subsubsection{Objective 3: Regulation of microRNA-mediated neuroprotective and fuel switching in} hypoxic H. glaber brains.

One of the master regulators of metabolic rate depression are miRNAs. These rapid and reversible short non-coding RNAs can broadly target all aspects of a cell's response to changing environmental conditions. This is critical during metabolic rate depression, when global reprogramming of gene expression is required to both suppress non-essential energy expensive processes as well as to maintain and activate processes needed for basic survival and protection. 
Therefore, is it likely that miRNAs are also involved in naked mole-rat hypoxia tolerance. This objective, explored in Chapter 4 of this thesis, aims to use next generation small RNA sequencing to comprehensively characterize the differential regulation of miRNAs in hypoxic brains. This is followed up with a combination of bioinformatic analyses to predict the downstream genes and pathways that these miRNAs target, and both RT-qPCR and western blotting will be used to validate these interactions during hypoxia.

\subsubsection{Objective 4: Explore AMPK-driven metabolic reorganization and reprioritization in}

\section{hypoxic H. glaber skeletal muscles.}

To cope with hypoxia, naked mole-rats reduce their metabolic requirements, a strategy that all hypometabolic organisms have evolved. Currently, the mechanism that naked mole-rats use to facilitate this response has yet to be determined. A possible candidate for this regulation is AMPK, a central energy sensor that can modulate a cell's primary energy source and mediate a switch between carbohydrate and lipid metabolism as needed. To that end, this objective, investigated in Chapter 5 of this thesis, uses RT-qPCR to examine the differential expression of AMPK targeting of miRNAs during hypoxia. Western blotting is also used to determine AMPK regulation and posttranslational modification, as well as differential abundance of glucose transporters, glycogen metabolism, and lipid metabolism proteins in the muscles of naked mole-rats to elucidate energy reprioritization between tissues when oxygen is limited. 


\subsection{References}

Andziak, B., Buffenstein, R., 2006. Disparate patterns of age-related changes in lipid peroxidation in long-lived naked mole-rats and shorter-lived mice. Aging Cell 5, 525-532.

Andziak, B., O’Connor, T.P., Qi, W., Dewaal, E.M., Pierce, A., Chaudhuri, A.R., Van Remmen, H., Buffenstein, R., 2006. High oxidative damage levels in the longest-living rodent, the naked mole-rat. Aging Cell 5, 463-471.

Bartel, D., 2004. MicroRNAs genomics, biogenesis, mechanism, and function. Cell 116, 281-297.

Bartel, D.P., 2009. MicroRNAs: target recognition and regulatory functions. Cell 136, 215-33.

Braude, S., Holtze, S., Begall, S., Brenmoehl, J., Burda, H., Dammann, P., del Marmol, D., Gorshkova, E., Henning, Y., Hoeflich, A., Höhn, A., Jung, T., Hamo, D., Sahm, A., Shebzukhov, Y., Šumbera, R., Miwa, S., Vyssokikh, M.Y., von Zglinicki, T., Averina, O., Hildebrandt, T.B., 2021. Surprisingly long survival of premature conclusions about naked mole-rat biology. Biol. Rev. 96, 376-393.

Brett, R.A., 1986. The ecology and behaviour of the naked mole-rat, Heterocephalus glaber Ruppell (Rodenti:Bathyergidae). University College London (University of London).

Chung, D., Dzal, Y.A., Seow, A., Milsom, W.K., Pamenter, M.E., 2016. Naked mole-rats exhibit metabolic but not ventilatory plasticity following chronic sustained hypoxia. Proc. R. Soc. B Biol. Sci. 283, 20160216.

Crish, S.D., Rice, F.L., Park, T.J., Comer, C.M., 2003. Somatosensory organization and behavior in naked mole-rats I: Vibrissa-like body hairs comprise a sensory array that mediates orientation to tactile stimuli. Brain. Behav. Evol. 62, 141-151.

De Waal, E.M., Liang, H., Pierce, A., Hamilton, R.T., Buffenstein, R., Chaudhuri, A.R., 2013. Elevated protein carbonylation and oxidative stress do not affect protein structure and function in the long-living naked-mole-rat: A proteomic approach. Biochem. Biophys. Res. Commun. 434, 815-819.

Ebert, M.S., Sharp, P.A., 2012. Roles for microRNAs in conferring robustness to biological processes. Cell 149, 515-24.

Edrey, Y.H., Medina, D.X., Gaczynska, M., Osmulski, P.A., Oddo, S., Caccamo, A., Buffenstein, R., 2013. Amyloid beta and the longest-lived rodent: The naked mole-rat as a model for natural protection from alzheimer's disease. Neurobiol. Aging 34, 2352-2360.

Edrey, Y.H., Oddo, S., Cornelius, C., Caccamo, A., Calabrese, V., Buffenstein, R., 2014. Oxidative damage and amyloid- $\beta$ metabolism in brain regions of the longest-lived rodents. J. Neurosci. Res. 92, 195-205.

Faulkes, C., Bennet, N., 2000. African Mole-Rats: Ecology and Eusociality. Ecology. 
Friedman, R.C., Farh, K.K.-H., Burge, C.B., Bartel, D.P., 2008. Most mammalian mRNAs are conserved targets of microRNAs. Genome Res. 19, 92-105.

Giraud-Billoud, M., Rivera-Ingraham, G.A., Moreira, D.C., Burmester, T., Castro-Vazquez, A., Carvajalino-Fernández, J.M., Dafre, A., Niu, C., Tremblay, N., Paital, B., Rosa, R., Storey, J.M., Vega, I.A., Zhang, W., Yepiz-Plascencia, G., Zenteno-Savin, T., Storey, K.B., HermesLima, M., 2019. Twenty years of the 'Preparation for Oxidative Stress' (POS) theory: Ecophysiological advantages and molecular strategies. Comp. Biochem. Physiol. Part A Mol. Integr. Physiol. 234, 36-49.

Grimes, K.M., Voorhees, A., Chiao, Y.A., Han, H.C., Lindsey, M.L., Buffenstein, R., 2014. Cardiac function of the naked mole-rat: Ecophysiological responses to working underground. Am. J. Physiol. - Hear. Circ. Physiol. 306.

Hadj-Moussa, H., Storey, K.B., 2020. The OxymiR response to oxygen limitation: A comparative microRNA perspective. J. Exp. Biol.

Hadj-Moussa H, Hawkins LJ, \& Storey KB. 2021. Role of MicroRNAs in Extreme Animal Survival Strategies. MiRNomics II: MicroRNA Biology and Computational Analysis. Springer Science. Book Chapter. In press.

Hardie, D.G., Ross, F.A., Hawley, S.A., 2012. AMPK: A nutrient and energy sensor that maintains energy homeostasis. Nat. Rev. Mol. Cell Biol.

Hawkins, L.J., Hadj-Moussa, H., Nguyen, V.C., Pamenter, M.E., Storey, K.B., 2019. Naked molerats activate neuroprotective proteins during hypoxia. J. Exp. Zool. Part A Ecol. Integr. Physiol. 331, 571-576.

He, L., Hannon, G.J., 2004. MicroRNAs: Small RNAs with a big role in gene regulation. Nat. Rev. Genet. 5, 522-531.

Heffner, R.S., Heffner, H.E., 1993. Degenerate hearing and sound localization in naked mole-rats (Heterocephalus glaber), with an overview of central auditory structures. J. Comp. Neurol. 331, 418-433.

Hetling, J.R., Baig-Silva, M.S., Comer, C.M., Pardue, M.T., Samaan, D.Y., Qtaishat, N.M., Pepperberg, D.R., Park, T.J., 2005. Features of visual function in the naked mole-rat Heterocephalus glaber. J. Comp. Physiol. A Neuroethol. Sensory, Neural, Behav. Physiol. 191, 317-330.

Hilton, H.G., Rubinstein, N.D., Janki, P., Ireland, A.T., Bernstein, N., Fong, N.L., Wright, K.M., Smith, M., Finkle, D., Martin-McNulty, B., Roy, M., Imai, D.M., Jojic, V., Buffenstein, R., 2019. Single-cell transcriptomics of the naked molerat reveals unexpected features of mammalian immunity. PLoS Biol. 17.

Hochachka, P.W., Buck, L.T., Doll, C.J., Land, S.C., 1996. Unifying theory of hypoxia tolerance: molecular/metabolic defense and rescue mechanisms for surviving oxygen lack. Proc. Natl. Acad. Sci. U. S. A. 93, 9493-8. 
Holtze, S., Braude, S., Lemma, A., Koch, R., Morhart, M., Szafranski, K., Platzer, M., Alemayehu, F., Goeritz, F., Hildebrandt, T.B., 2018. The microenvironment of naked mole-rat burrows in East Africa. Afr. J. Ecol. 56, 279-289.

Huang, Y., Shen, X.J., Zou, Q., Wang, S.P., Tang, S.M., Zhang, G.Z., 2011. Biological functions of microRNAs: a review. J. Physiol. Biochem. 67, 129-39.

Jarvis, J.U.M., Sherman, P.W., 2002. Heterocephalus glaber. Mamm. Species 3, 1-9.

Kahn, B.B., Alquier, T., Carling, D., Hardie, D.G., 2005. AMP-activated protein kinase: Ancient energy gauge provides clues to modern understanding of metabolism. Cell Metab.

Kim, E.B., Fang, X., Fushan, A.A., Huang, Z., Lobanov, A. V., Han, L., Marino, S.M., Sun, X., Turanov, A.A., Yang, P., Yim, S.H., Zhao, X., Kasaikina, M. V., Stoletzki, N., Peng, C., Polak, P., Xiong, Z., Kiezun, A., Zhu, Y., Chen, Y., Kryukov, G. V., Zhang, Q., Peshkin, L., Yang, L., Bronson, R.T., Buffenstein, R., Wang, B., Han, C., Li, Q., Chen, L., Zhao, W., Sunyaev, S.R., Park, T.J., Zhang, G., Wang, J., Gladyshev, V.N., 2011. Genome sequencing reveals insights into physiology and longevity of the naked mole-rat. Nature 479, 223-227.

Kirby, A.M., Fairman, G.D., Pamenter, M.E., 2018. Atypical behavioural, metabolic and thermoregulatory responses to hypoxia in the naked mole-rat (Heterocephalus glaber). J. Zool. 305, 106-115.

Krivoruchko, A., Storey, K.B., 2015. Turtle anoxia tolerance: Biochemistry and gene regulation. Biochim. Biophys. Acta - Gen. Subj. 1850, 1188-1196.

Krivoruchko, A., Storey, K.B., 2010. Forever young: mechanisms of natural anoxia tolerance and potential links to longevity. Oxid. Med. Cell. Longev. 3, 186-98.

Larson, J., Drew, K.L., Folkow, L.P., Milton, S.L., Park, T.J., 2014. No oxygen? No problem! intrinsic brain tolerance to hypoxia in vertebrates. J. Exp. Biol.

Lewis, K.N., Andziak, B., Yang, T., Buffenstein, R., 2013. The naked mole-rat response to oxidative stress: Just deal with it. Antioxidants Redox Signal. 19, 1388-1399.

Lewis, K.N., Mele, J., Hayes, J.D., Buffenstein, R., 2010. Nrf2, a guardian of healthspan and gatekeeper of species longevity, in: Integrative and Comparative Biology. Integr Comp Biol, pp. 829-843.

Lewis, K.N., Wason, E., Edrey, Y.H., Kristan, D.M., Nevo, E., Buffenstein, R., 2015. Regulation of Nrf2 signaling and longevity in naturally long-lived rodents. Proc. Natl. Acad. Sci. U. S. A. $112,3722-3727$.

Lipman, R., Galecki, A., Burke, D.T., Miller, R.A., 2004. Genetic loci that influence cause of death in a heterogeneous mouse stock. Journals Gerontol. - Ser. A Biol. Sci. Med. Sci. 59, 977983.

McNab, B.K., 1979. The Influence of Body Size on the Energetics and Distribution of Fossorial 
and Burrowing Mammals. Ecology 60, 1010-1021.

Mitchell, T.W., Buffenstein, R., Hulbert, A.J., 2007. Membrane phospholipid composition may contribute to exceptional longevity of the naked mole-rat (Heterocephalus glaber): A comparative study using shotgun lipidomics. Exp. Gerontol. 42, 1053-1062.

Mooney, S.J., Coen, C.W., Holmes, M.M., Beery, A.K., 2015. Region-specific associations between sex, social status, and oxytocin receptor density in the brains of eusocial rodents. Neuroscience 303, 261-269.

Munro, D., Baldy, C., Pamenter, M.E., Treberg, J.R., 2019. The exceptional longevity of the naked mole-rat may be explained by mitochondrial antioxidant defenses. Aging Cell 18, e12916.

Nguyen, V.C., Deck, C.A., Pamenter, M.E., 2019. Naked mole-rats reduce the expression of ATPdependent but not ATP-independent heat shock proteins in acute hypoxia. J. Exp. Biol. 222.

Okanoya, K., Yosida, S., Barone, C.M., Applegate, D.T., Brittan-Powell, E.F., Dooling, R.J., Park, T.J., 2018. Auditory-vocal coupling in the naked mole-rat, a mammal with poor auditory thresholds. J. Comp. Physiol. A Neuroethol. Sensory, Neural, Behav. Physiol. 204, 905-914.

Orr, M.E., Garbarino, V.R., Salinas, A., Buffenstein, R., 2016. Extended postnatal brain development in the longest-lived rodent: Prolonged maintenance of neotenous traits in the naked mole-rat brain. Front. Neurosci. 10.

Pamenter, M.E., Dzal, Y.A., Thompson, W.A., Milsom, W.K., 2019. Do naked mole-rats accumulate a metabolic acidosis or an oxygen debt in severe hypoxia? J. Exp. Biol. 222.

Pamenter, M.E., Hall, J.E., Tanabe, Y., Simonson, T.S., 2020. Cross-Species Insights Into Genomic Adaptations to Hypoxia. Front. Genet.

Pamenter, M.E., Lau, G.Y., Richards, J.G., Milsom, W.K., 2018. Naked mole-rat brain mitochondria electron transport system flux and $\mathrm{H}+$ leak are reduced during acute hypoxia. J. Exp. Biol. 221.

Park, T.J., Lu, Y., Jüttner, R., Smith, E.S.J., Hu, J., Brand, A., Wetzel, C., Milenkovic, N., Erdmann, B., Heppenstall, P.A., Laurito, C.E., Wilson, S.P., Lewin, G.R., 2008. Selective inflammatory pain insensitivity in the African naked mole-rat (Heterocephalus glaber). PLoS Biol. 6, 0156-0170.

Park, T.J., Reznick, J., Peterson, B.L., Blass, G., Omerbašić, D., Bennett, N.C., Kuich, P.H.J.L., Zasada, C., Browe, B.M., Hamann, W., Applegate, D.T., Radke, M.H., Kosten, T., Lutermann, H., Gavaghan, V., Eigenbrod, O., Bégay, V., Amoroso, V.G., Govind, V., Minshall, R.D., Smith, E.S.J., Larson, J., Gotthardt, M., Kempa, S., Lewin, G.R., 2017. Fructose-driven glycolysis supports anoxia resistance in the naked mole-rat. Science 356, 307-311.

Pérez, V.I., Buffenstein, R., Masamsetti, V., Leonard, S., Salmon, A.B., Mele, J., Andziak, B., Yang, T., Edrey, Y., Friguet, B., Ward, W., Richardson, A., Chaudhuri, A., 2009. Protein 
stability and resistance to oxidative stress are determinants of longevity in the longest-living rodent, the naked mole-rat. Proc. Natl. Acad. Sci. U. S. A. 106, 3059-3064.

Rider, M.H., 2016. Role of AMP-activated protein kinase in metabolic depression in animals. J. Comp. Physiol. B Biochem. Syst. Environ. Physiol. https://doi.org/10.1007/s00360-0150920-x

Shebzukhov, Y., Holtze, S., Hirseland, H., Schäfer, H., Radbruch, A., Hildebrandt, T., Grützkau, A., 2019. Identification of cross-reactive antibodies for the detection of lymphocytes, myeloid cells and haematopoietic precursors in the naked mole-rat. Eur. J. Immunol. 49, 2103-2110.

Storey, K.B., 2015. Regulation of hypometabolism: insights into epigenetic controls. J. Exp. Biol. $218,150-159$.

Toor, I., Edwards, P.D., Kaka, N., Whitney, R., Ziolkowski, J., Monks, D.A., Holmes, M.M., 2020. Aggression and motivation to disperse in eusocial naked mole-rats, Heterocephalus glaber. Anim. Behav. 168, 45-58.

Winder, W.W., Hardie, D.G., Mustard, K.J., Greenwood, L.J., Paxton, B.E., Park, S.H., Rubink, D.S., Taylor, E.B., 2003. Long-term regulation of AMP-activated protein kinase and acetylCoA carboxylase in skeletal muscle, in: Biochemical Society Transactions. Portland Press Ltd, pp. 182-185. 


\section{Chapter 2}

\section{Oxidative Damage: Mutli-tissue profile}




\section{Naked mole-rats resist the accumulation of hypoxia-induced oxidative damage}

Hanane Hadj-Moussa ${ }^{1}$, Liam Eaton ${ }^{2, \#}$, Hang Cheng ${ }^{2, \#}$, Matthew E. Pamenter ${ }^{2,3}$, and Kenneth B. Storey ${ }^{1}$

${ }^{1}$ Department of Biology and Institute of Biochemistry, Carleton University, Ottawa, ON, Canada

2 Biology Department, University of Ottawa, Ottawa, ON, Canada

${ }^{3}$ Brain and Mind Research Institute, University of Ottawa, Ottawa, ON, Canada

\# Equal contribution 


\subsection{Abstract}

Naked mole-rats are among the few mammals with the ability to endure severe hypoxia. These unique rodents use metabolic rate depression along with various molecular mechanisms to successfully overcome the challenges of oxygen-limitation, which they experience in their underground borrows. While studies have reported that naked mole-rats exhibit inherently higher levels of oxidative damage across their lifespan as compared to mice, it has yet to be determined whether naked mole-rats are vulnerable to oxidative damage during periods of low oxygen exposure. To investigate this phenomenon, we examined cellular oxidative damage markers of macromolecules: DNA oxidation determined as 8-oxo-2'deoxyguanosine (8-OHdG) levels, RNA oxidation as 8 -hydroxyguanosine $(8-\mathrm{OHG})$, protein carbonylation, and lipid peroxidation in normoxic (control), or acute $\left(4 \mathrm{hr}\right.$ at $\left.7 \% \mathrm{O}_{2}\right)$ or chronic $\left(24 \mathrm{hr}\right.$ at $\left.7 \% \mathrm{O}_{2}\right)$ hypoxia-exposed naked mole-rats. Brain appears to be the most resilient organ to hypoxia-induced oxidative damage, with both brain and heart exhibiting enhanced antioxidant capacity during hypoxia. Levels of DNA and RNA oxidation were minimally changed in all tissues and no changes were observed in protein carbonylation. Most tissues experienced lipid peroxidation, with liver displaying a 9.6-fold increase during hypoxia. Concomitantly, levels of DNA damage repair proteins were dynamically regulated in a tissue-specific manner, with white adipose displaying a significant reduction during hypoxia. Our findings show that naked mole-rats largely avoid hypoxia-induced oxidative damage, possibly due to their high tolerance to redox stress, or to reduced oxidative requirements made possible during their hypometabolic response when oxygen supply is limited. 


\subsection{Introduction}

Naked mole-rats (Heterocephalus glaber) are unique mammals that show high tolerances for oxygen-deprivation (Chung et al., 2016) and chemical pain (Browe et al., 2020), as well as high resistance to both cancer (Shepard and Kissil, 2020), and neurodegeneration (Orr et al., 2015). Added to this is their apparent lack of ageing-associated physiological decline, with mortality rates remaining stable with age, rendering them the longest-lived rodents (Edrey et al., 2011; Smith et al., 2015). The exceptional longevity of naked mole-rats is surprising, up to 32 years, especially when considering that not only do both older and age-matched naked mole-rats accumulate higher levels of oxidative damage compared to mice, but that they also have less efficient cellular repair mechanisms (Lewis et al., 2013). Other long-lived species from birds to bats also exhibit elevated levels of oxidative damage, starting at a young age (Rodriguez et al., 2011). These animals contradict the oxidative theory of ageing, which postulates that ageing is associated with macromolecular damage caused by deleterious products of oxidative metabolism (Zuo et al., 2020). To date, work on oxidative stress responses in naked mole-rats has only examined oxidative damage and repair in the context of ageing which led to our main question. Do episodes of oxygen deprivation impact oxidative damage accumulation in naked mole-rats?

Cycling between normoxic and hypoxic conditions can lead to elevation of reactive oxygen species (ROS) and reactive nitrogen species (RNS) production. Low concentrations of ROS serve as second messengers that activate redox-sensitive signalling pathways and can regulate a variety of cellular processes (Saldmann et al., 2019). However, accumulation of by-products of aerobic metabolism can have detrimental effects as they irreversibly oxidize macromolecules, injure cells, and render them dysfunctional. Naked mole-rats can tolerate repeated bouts of hypoxiareoxygenation, and one of the central adaptations they use to mitigate oxidative stress is a global 
reduction in metabolic rate as this facilitates a decrease in ATP expenditure and improvement in energy efficiency (Pamenter et al., 2019). For example, naked mole-rat brains were found to maintain nitric oxide homeostasis during hypoxia and ischemia (oxygen and glucose deprivation) and avoid bursts of nitric oxide production during reoxygenation (Wang et al., 2020). Oxidative stress is typically assessed by monitoring damage markers since ROS are very short-lived but cause structural damage to macromolecules, including peroxidation of fatty acids, modification of protein carbonyl groups, and the formation of double bond adducts on nucleic acids resulting in 8oxo-2'deoxyguanosine (8-OHdG) on DNA (leading to deleterious gene mutations) and the formation of 8-hydroxyguanosine (8-OHG) on RNA (Lewis et al., 2013). Elevated levels of oxidative damage have been partly attributed to the mole-rat's high intracellular iron content that can ravage cells with redox reactions (Buffenstein, 2005). This phenomenon has also been observed in other long-lived animals including bats and birds that show higher levels of DNA oxidative damage, as compared with their short-lived counterparts (Lewis et al., 2013). Yet curiously, when naked mole-rat livers were examined, they showed significantly reduced activities of antioxidant enzymes including CuZnSOD, catalase, glutathione peroxidase, glutathione reductase as well as lower levels of thioredoxin and glutathione, as compared to mice (Lewis et al., 2013). Only MnSOD was found to display a slight increase over the activity found in mouse liver, this is in combination with naked mole-rat heart and muscle exhibiting higher mitochondrial ROS scavenging capacity, relative to mice (Lewis et al., 2013; Munro et al., 2019).

In crowded underground colonies, eusocial naked mole-rats putatively experience both hypoxia and hypercapnia within their nest chambers (Holtze et al., 2018). However, whereas the degree of oxygen-deprivation experienced in their natural habitat is disputed, laboratory studies have demonstrated that naked mole-rats can withstand hypoxia exposures for days to weeks at $8 \%$ 
$\mathrm{O}_{2}$ levels, hours at $3 \% \mathrm{O}_{2}$, and up to 18 mins in complete anoxia $\left(0 \% \mathrm{O}_{2}\right)($ Chung et al., 2016; Pamenter et al., 2018; Park et al., 2017). Despite their inherently lower resting metabolic rate, as compared to mice of the same size, naked mole-rats further depress their metabolic rate and physiological parameters including breathing frequency, heart rate, activity, and body temperature to match reduced oxygen availability (Pamenter et al., 2019; Zuo et al., 2020). This is facilitated by a shift to carbohydrate fuel sources and anaerobic pathways, a strategy common to hypoxia and anoxia tolerant organisms that combine global metabolic rate depression of processes that are nonessential for basic survival such as cell growth and de novo protein synthesis (Al-attar et al., 2020; Krivoruchko and Storey, 2010), coupled with the activation of redundant pro-survival and antioxidant defense mechanisms to protect against the cellular challenges associated with oxygendeprivation and reoxygenation (Hochachka et al., 1996). Examples include the activation of prosurvival transcription factors (HIF-1 $\alpha$ and NFkB) (Hawkins et al., 2019; Xiao et al., 2017b), antiapoptotic mechanisms (Xiao et al., 2017a), heat shock proteins for proteome stabilization (Nguyen et al., 2019), neuroprotective factors (Hawkins et al., 2019), and switching to anaerobic fuel sources (Hadj-Moussa et al., 2021; Park et al., 2017), much of this metabolic reorganization being facilitated by epigenetic, post-transcriptional, and post-translational mechanisms.

The question we sought to answer was, does hypoxia exposure impact levels of oxidative damage and repair in naked mole-rats? To explore this, we examined the levels of irreversibly oxidized macromolecules, 8-OHdG DNA, 8-OHG RNA, protein carbonyls, and lipids peroxides under normoxia $\left(21 \% \mathrm{O}_{2}\right)$, short-term/acute hypoxia $\left(4 \mathrm{hr}\right.$ at $\left.7 \% \mathrm{O}_{2}\right)$, and long-term/chronic hypoxia ( $24 \mathrm{hr}$ at $7 \% \mathrm{O}_{2}$ ) exposures in six central naked mole-rat organs (whole brain, heart, liver, skeletal muscle, white adipose tissue (WAT) and kidney). We also explored the underlying mechanism of putative redox damage in each tissue by assessing total antioxidant capacities in 
normoxic and hypoxic naked mole-rats and central DNA damage repair proteins. With naked mole-rats emerging as a unique and natural animal model for hypoxia, ageing, cancer, and neurodegenerative research, dissecting their oxidative stress profiles and ability to combat or circumvent the challenges associated with short and prolonged oxygen limitation will provide insights that further our understanding of their physiology and explore the relevance of naked mole-rats for use in biomedical research into ischemia-caused human damage and disease.

\subsection{Materials and Methods}

\subsection{1 $\quad \underline{\text { Animals }}$}

Twelve subordinate, non-breeding naked mole-rat adult males were obtained from a grouphoused colony at the University of Ottawa. Animals lived in interconnected multi-cage systems at $30^{\circ} \mathrm{C}$ and $21 \% \mathrm{O}_{2}$ in $50 \%$ humidity with a $12 \mathrm{~L}: 12 \mathrm{D}$ light cycle and were fed fresh tubers, vegetables, fruit and Pronutro cereal supplement ad libitum. For additional information on animal feeding, monitoring, and behaviour, refer to Houlahan et al. (2018). Naked mole-rats were randomly divided into three groups $(n=4)$ and placed into three separate experimental chambers, each with a thin layer of bedding on the floor. Chambers were sealed and ventilated with the desired fractional gas composition by calibrated rotameters (Praxair, Mississauga, ON, CA). Inflowing gas was provided at a flow rate of $0.5 \mathrm{~L} / \mathrm{min}$, as assessed by a calibrated mass flow meter (Alicat Scientific, Tucson, AZ, USA). Normoxic control treatments were as followed $\left(20.95 \% \mathrm{O}_{2}, 0.05 \% \mathrm{CO}_{2}\right.$, balance $\mathrm{N}_{2}$ ). Oxygen levels were continuously monitored throughout the experiment using a FC-10 $\mathrm{O}_{2}$ analyzer (Sable Systems International, Las Vegas, NV, USA). 
Hypoxia exposure experiments were performed as follows. For $4 \mathrm{~h}$ acute hypoxia exposures, incurrent gas was switched to $7 \% \mathrm{O}_{2}$ (with $0.05 \% \mathrm{CO}_{2}$, balance $\mathrm{N}_{2}$ ) and flow rates were increased to $1 \mathrm{~L} / \mathrm{min}$ for $10 \mathrm{~min}$ to rapidly reduce the $\mathrm{O}_{2}$ content of the chamber to $7 \% \mathrm{O}_{2}$ and then returned to $0.5 \mathrm{~L} / \mathrm{min}$ for the remaining $4 \mathrm{~h}$ of exposure. Animals in the chronic $24 \mathrm{~h}$ hypoxia exposure group were treated as above but left in the $7 \% \mathrm{O}_{2}$ chambers for 24 hours.

Animals in all experimental groups were euthanized by conscious cervical dislocation, immediately followed by decapitation. Tissues (whole brain, heart, liver, temporalis muscle, kidneys, and WAT were rapidly dissected within $30 \mathrm{sec}$, immediately flash frozen in liquid nitrogen, and stored at $-80^{\circ} \mathrm{C}$ until analysis.

All naked mole-rat experiments were conducted in compliance with the requirements of the Canadian Council on Animal Care and were approved by the University of Ottawa Animal Care Committee (protocol \#2535).

\subsubsection{Soluble protein isolation}

For the antioxidant capacity and protein carbonylation assays, the following protein extraction was performed as per the manufacturer's instructions in the test kit (EMD Millipore; 48630MAG). Briefly, $75 \mathrm{mg}$ of frozen tissue was homogenized 1:5 w/v in 1X lysis buffer (EMD Millipore, 43-045). Lysis buffer was pre-chilled and combined with $1 \mathrm{mM} \mathrm{Na}_{3} \mathrm{VO}_{4}, 10 \mathrm{mM} \mathrm{NaF}$, $10 \mathrm{mM} \beta$-glycerophosphate, and $10 \mu \mathrm{L} / \mathrm{mL}$ of protease inhibitor cocktail containing $104 \mathrm{mM}$ AEBSF, $80 \mu \mathrm{M}$ aprotinin, $4 \mathrm{mM}$ bestatin, $1.4 \mathrm{mM}$ E-64 protease inhibitor, $2 \mathrm{mM}$ leupeptin, 1.5 mM pepstatin A (Bioshop, PIC001.1). Homogenates were chilled on ice for 30 min with intermittent vortexing every $10 \mathrm{~min}$. Next, samples were centrifuged at $14,000 \mathrm{x}$ g for $20 \mathrm{~min}$ at 4 
${ }^{\circ} \mathrm{C}$. Supernatants containing soluble protein were collected, aliquoted, and stored at $-80{ }^{\circ} \mathrm{C}$ until later use.

The thiobarbituric acid reactive substances (TBARS) assay kit required the following specialized protein extraction protocol. PBS buffer was prepared with $25 \mathrm{~g} \mathrm{KCl}$ and $2.8 \mathrm{~g} \mathrm{Na}_{2} \mathrm{HPO}_{4}$ in $0.313 \mathrm{~L}$ of distilled water to obtain a $0.01 \mathrm{mM}$ PBS solution [pH 7.4]. Approximately $100 \mathrm{mg}$ of frozen tissue was thawed on ice and then combined with $900 \mu \mathrm{L}$ mL PBS buffer mixed (1:10) with heparin (Upjohn; Cat\# 009-0268-01). The purpose of heparin was to reduce haemoglobin interference in subsequent steps of the assay. Samples were briefly homogenized and then centrifuged at $10,000 \mathrm{xg}$ for $10 \mathrm{~min}$ at $4{ }^{\circ} \mathrm{C}$ to collect the protein containing supernatant. Supernatants were the stored at $-80{ }^{\circ} \mathrm{C}$ for until later use.

Protein concentrations were measured using the Bradford assay with a BSA standard (BioRad; 500-0006) and adjusted to the appropriate concentrations for each assay as described below.

\subsubsection{DNA isolation}

DNA was extracted from all normoxic and 4 and 24 h hypoxic naked mole-rat tissues $(n=4)$ using a Quick-DNA Plus Kit (Zymo Research, Cat\# D4068) as per the manufacturer's instructions. Frozen tissue samples ( $\sim 25 \mathrm{mg}$ ) were briefly homogenized in proteinase K solution (47.5\% solid tissue buffer, $5 \%$ proteinase K) using a glass pestle and digested overnight. Then $400 \mu \mathrm{L}$ of genomic binding buffer was mixed with each sample and vortexed for $15 \mathrm{sec}$. Each sample was then transferred to a Zymo-Spin column and centrifuged at 12,000 g for $1 \mathrm{~min}$. Flow through was discarded and the column was washed with $400 \mu \mathrm{L}$ of DNA pre-wash buffer. Columns were centrifuged again and washed with $900 \mu \mathrm{L}$ of g-DNA wash buffer. DNA was then eluted from each column after a 5 min incubation in $50 \mu \mathrm{L}$ of DNA elution buffer. 


\subsection{4 $\quad \underline{\text { Total RNA isolation }}$}

RNA isolation was performed on approximately $50 \mathrm{mg}$ of tissue ( $n=4$ individual animals) for normoxic, $4 \mathrm{~h}$ hypoxic, and $24 \mathrm{~h}$ hypoxic naked mole-rat groups. Total RNA was isolated using the Trizol-chloroform (Invitrogen; Cat\# 15596-018) method as per the manufacturers instruction and as described in Hadj-Moussa et al. (2020). Extracted total RNA pellets were resuspended in $50 \mu \mathrm{L}$ RNase-free water and both RNA $260 / 280$ purity ratio and concentration were assessed using a Take3 micro-volume plate and spectrophotometer (BioTek, Winooski, VT, USA). Integrity of the RNA samples was examined by verifying the presence of sharp bands for $28 \mathrm{~S}$ and $18 \mathrm{~S}$ rRNA using gel electrophoresis on a 1\% agarose gel with SYBR Green (Invitrogen; Cat\# S7563).

\subsubsection{Protein carbonylation kit}

Proteins isolated with the protocol stated above in Section 2.4. were treated with streptomycin sulfate (Cayman Chemical; Cat\# 21211) to remove nucleic acids as they erroneously contribute to a higher estimation of carbonyls. Samples were incubated at room temperature for 15 min with a $1 \%$ final concentration of streptomycin sulfate from the following stock solution (10\% streptomycin sulfate, $50 \mathrm{mM} \mathrm{KH}_{2} \mathrm{PO}_{4}, \mathrm{pH}$ 7.2). Samples were then centrifuged at 6,000 $\mathrm{g}$ for 10 min at $4{ }^{\circ} \mathrm{C}$. This supernatant was used to assess protein carbonyl content. Samples were adjusted to a final concentration of $4 \mu \mathrm{g} / \mu \mathrm{L}$ using $1 \mathrm{X}$ lysis buffer.

The Cayman Chemical Protein carbonylation assay kit was used (Cat\# 10005020) as per the manufacturer's instructions. This kit measures the amount of protein-hydrazone produced from the reaction of 2,4-dinitrophenylhydrazine (DNPH) with protein carbonyls in tissue homogenates which is then quantified spectrophotometrically as a measure of protein carbonyl content. Each standardized naked mole-rat sample was divided into two $100 \mu \mathrm{L}$ aliquots, where one was treated with $400 \mu \mathrm{L}$ of DNPH (experimental) and the other was treated with $400 \mu \mathrm{L}$ of $2.5 \mathrm{M} \mathrm{HCl}$ 
(control). Both experimental and control samples were incubated at room temperature for 1 hour in the dark, with vortexing every $15 \mathrm{~min}$. Samples were then mixed with $500 \mu \mathrm{L}$ of $20 \%$ trichloroacetic acid (TCA), vortexed, incubated for $5 \mathrm{~min}$ on ice, and centrifuged at $10,000 \mathrm{~g}$ for 10 min at $4{ }^{\circ} \mathrm{C}$. Supernatants were discarded and pellets were resuspended in $500 \mu \mathrm{L}$ of $10 \% \mathrm{TCA}$, vortexed, incubated on ice for $5 \mathrm{~min}$, and centrifuged again. Supernatants were discarded, pellets were resuspended in $500 \mu \mathrm{L}$ 1:1 v:v ethanol/ethyl acetate, vortexed, incubated on ice, and centrifuged. This step was repeated twice more. Pellets were then resuspended in $250 \mu \mathrm{L}$ of $\mathrm{HCl}$, and centrifuged. For the assay, $220 \mu \mathrm{L}$ of supernatant from each sample (experimental and control) were transferred into separate wells in a 96-well microplate and absorbance was measured at 370 $\mathrm{nm}$. The optical density values of experimental samples were adjusted by subtracting the corresponding value of the control sample. Protein carbonyl concentrations $(\mathrm{nmol} / \mathrm{mL})$ were then calculated as per the manufacturer's instructions.

\subsubsection{DNA and RNA oxidative damage assays}

Isolated RNA ( $4 \mu \mathrm{g})$ and DNA $(\sim 1 \mu \mathrm{g})$ from normoxic, $4 \mathrm{~h}$ hypoxic, and $24 \mathrm{~h}$ hypoxic naked mole-rat tissues were incubated at $55^{\circ} \mathrm{C}$ for $1 \mathrm{~h}$ with $10 \mathrm{U}$ of nuclease P1 (Millipore-Sigma, Cat\# N8630) per sample. To digest nucleotides to nucleosides, $1 \mathrm{U}$ of alkaline phosphatase (NEB, Cat\# M0290S) was added to each sample and incubated at $37^{\circ} \mathrm{C}$ for $30 \mathrm{~min}$, then boiled for $10 \mathrm{~min}$, and placed on ice until use.

The DNA/RNA oxidative damage ELISA (Cayman Chemical, Cat\# 589320) was performed as per the manufacturer's instructions. A DNA/RNA oxidative damage standard curve was generated using the reagents supplied in the kit with concentrations ranging from 10.3 to 3000 $\mathrm{pg} / \mathrm{mL}$. RNA samples were each diluted in 1 X ELISA buffer (supplied with the kit) to $12 \mathrm{ng} / \mu \mathrm{L}$. DNA samples were each diluted in $1 \mathrm{X}$ ELISA buffer to the following: Brain $=15 \mathrm{ng} / \mu \mathrm{L}$, Heart $=$ 
$15 \mathrm{ng} / \mu \mathrm{L}, \mathrm{WAT}=10 \mathrm{ng} / \mu \mathrm{L}$, Kidney $=15 \mathrm{ng} / \mu \mathrm{L}$, Skeletal muscle $=15 \mathrm{ng} / \mu \mathrm{L}$, and Liver $=12$ $\mathrm{ng} / \mu \mathrm{L}$.

Blanks were prepared as follows: A non-specific binding blank (100 $\mu$ L 1X ELISA buffer, 50 $\mu \mathrm{L}$ tracer dye), a maximum binding blank (50 $\mu \mathrm{L} 1 \mathrm{X}$ ELISA buffer, $50 \mu \mathrm{L}$ tracer and $50 \mu \mathrm{L}$ of monoclonal antibody) and a background absorbance blank (Ellman's reagent) were used. For all three conditions and naked mole-rat samples, wells were prepared as follows: $50 \mu \mathrm{L}$ of each DNA/RNA sample, AChE tracer, and monoclonal antibody specific DNA/RNA damage markers were combined into each well of a 96-well microplate. The microplate was then covered and incubated for $18 \mathrm{~h}$ in the dark on a shaker at $4{ }^{\circ} \mathrm{C}$. Wells were then washed five times with $1 \mathrm{X}$ wash buffer. Then, $200 \mu \mathrm{L}$ of Ellman's reagent was added to each well, the plate was covered and incubated for 90 min on a shaker at room temperature (for the RNA kit, muscle was incubated for $120 \mathrm{~min})$. Absorbance was measured spectrophotometrically at $410 \mathrm{~nm}$. All OD values were subtracted by the blank.

\subsubsection{Thiobarbituric Acid Reactive Substances (TBARS) assay}

To measure lipid peroxidation levels in naked mole-rat tissues, the R\&D Systems TBARS Parameter Assay Kit (Cat\# KGE013) was used as per the manufacturer's instructions. The measure of TBARS is a common method of determining relative lipid peroxide content. In the presence of heat and acid, malondialdehyde (MDA) reacts with thiobarbituric acid (TBA) to produce colored end-product that absorbs light at $532 \mathrm{~nm}$ that corresponds with the level of lipid peroxidation in the sample.

Naked mole-rat samples were prepared as described above. All samples required an acid treatment prior to assay: $300 \mu \mathrm{L}$ of sample were mixed with $300 \mu \mathrm{L}$ of TBARS Acid Reagent in a 
microcentrifuge tube. Samples were incubated for $15 \mathrm{~min}$ at room temperature and centrifuged at 12,000 g for $4 \mathrm{~min}$. Supernatants were transferred to clean microcentrifuge tubes and assayed immediately. For the assay, $150 \mu \mathrm{L}$ of samples was added to wells of a 96-well microplate and combined with $75 \mu \mathrm{L}$ of TBA reagent. Optical density of each well was then measured at $532 \mathrm{~nm}$. The microplate was covered and incubated for 3 hours at $45-50{ }^{\circ} \mathrm{C}$, except for liver samples that were incubated for 2.5 hours. Optical density was then measured again at $532 \mathrm{~nm}$ and pre-read optical densities were subtracted from the final reading to correct for the sample's contribution to the final absorption at $532 \mathrm{~nm}$. A standard curve of the acidified TBARS standard was generated with concentrations ranging from $167 \mu \mathrm{M}$ to $0.26 \mu \mathrm{M}$.

\subsubsection{Antioxidant capacity kit}

Antioxidant capacity was assayed using the Cayman Chemical ELISA kit (Cat\# 709001). This kit utilizes the ability of antioxidants in the sample to inhibit the oxidation of ABTS $\left[2,2^{\prime}\right.$-azinodi-(3-ethylbenzthiazoline sulfonate)] to $\mathrm{ABTS}^{+}$. Protein samples for this assay were standardized to $2 \mu \mathrm{g} / \mu \mathrm{L}$ by adding appropriate amounts of $1 \mathrm{X}$ antioxidant assay buffer provided with the kit. For this analysis, a standard curve was prepared by combining increasing amounts of Trolox (6hydroxy-2,5,7,8-tetramethylchoman-2-carboxylic acid), a water-soluble tocopherol analogue, diluted in antioxidant assay buffer, with concentrations ranging from 0 to $0.330 \mathrm{mM}$ Trolox.

For the assay, $10 \mu \mathrm{L}$ of Trolox standard or $2 \mu \mathrm{g} / \mu \mathrm{L}$ of protein samples was mixed with $10 \mu \mathrm{L}$ of metmyoglobin and $150 \mu \mathrm{L}$ of chromogen in a 96-well plate. Reactions were initiated with the addition of $40 \mu \mathrm{L}$ of $\mathrm{H}_{2} \mathrm{O}_{2}(441 \mu \mathrm{M})$. The reaction plate was then incubated on a shaker in the dark at room temperature. Absorbance was read at $750 \mathrm{~nm}$ and quantified as relative Trolox equivalents $(\mathrm{mM})$. 


\subsubsection{Relative abundance of DNA damage repair proteins}

A DNA damage/genotoxicity magnetic bead kit (EMD Millipore; 48-621MAG) was used to examine relative phosphorylation changes in Chk1 (Ser345), Chk2 (Thr68), H2A.X (Ser139) and p53 (Ser15) proteins as well as total protein levels of ATR, MDM2 and p21. For this kit, proteins isolated with the protocol stated in Section 2.4 were used for all six naked mole-rat tissues under normoxic, $4 \mathrm{~h}$ hypoxic, and $24 \mathrm{~h}$ hypoxic conditions ( $n=4 /$ independent animals per experimental condition). The Luminex assay was performed as per manufacturer's instructions.

HeLa Cell Lysate: Lambda phosphatase (Millipore; 47-229) was used as an unstimulated positive control. Jurkat cell lysate treated with Anisomycin (Millipore; 47-207) and A549 cell lysate treated with Camptothecin (Millipore; 47-218) were used as the stimulated controls. The following concentrations of tissue extracts were used for quantification; brain $=20 \mathrm{ng} / \mu \mathrm{L}$, heart $=$ $85 \mathrm{ng} / \mu \mathrm{L}, \mathrm{WAT}=20 \mathrm{ng} / \mu \mathrm{L}$, kidney $=40 \mathrm{ng} / \mu \mathrm{L}$, skeletal muscle $=40 \mathrm{ng} / \mu \mathrm{L}$, and liver $=85 \mathrm{ng} / \mu \mathrm{L}$.

Data acquisition was done using a Luminex 200 instrument (Luminex, Austin, TX) combined with Milliplex Analyst software (Millipore, Billerica, MA). The Luminex 200 settings were as follows: 50 events per bead, $100 \mu \mathrm{L}$ sample size, $8000-15,000$ gate settings, default (low PMT) reporter gains, and 60 seconds time out. Bead regions were: 28 for Chk1 (Ser345), 35 for Chk2 (Thr68), 39 for H2A.X (Ser139), 53 for p53 (Ser15), 21 for ATR, 67 for MDM2, and 75 for p21.

\subsubsection{0 $\quad \underline{\text { Statistical analysis }}$}

All assays were analyzed using a one-way ANOVA and a Dunnett's post-hoc test to compare normoxic, $4 \mathrm{~h}$ hypoxic, and $24 \mathrm{~h}$ hypoxic mole-rat conditions for each tissue, independently of one another ( $n=3$ - 4 independent biological replicates from different animals). RBioPlot was used to 
perform statistical analyses and to generate figures (Zhang and Storey, 2016), where statistical significance was defined as $p \leq 0.05$.

For each ELISA, the means \pm SEM of each condition (normoxic, $4 \mathrm{~h}$ hypoxic, and $24 \mathrm{~h}$ hypoxic) were calculated as per the manufacturer's instructions. Normoxic controls were then standardized to 1 and both means for each of the hypoxic conditions was expressed relative to the normoxic control mean.

\subsection{Results}

\subsubsection{Hypoxia has no effect on protein carbonylation levels}

Levels of protein carbonyls represent the extent of oxidative damage accrued by proteins. Protein carbonylation was not different between treatment conditions in any of the six naked molerat tissues examined (Figure 2.1).

\subsubsection{DNA oxidative damage is minimal during hypoxia}

Most naked mole-rat tissues and hypoxia exposures examined exhibited no changes in DNA oxidative damage (Figure 2.2). DNA oxidative damage did not occur in any condition or tissue, except in kidney, where DNA oxidative damage was increased by 1.54-fold and 1.92-fold, respectively (Figure 2.2).

\subsection{3 $\quad$ RNA oxidative damage only observed in kidney}

RNA oxidative damage decreased in three tissues during hypoxia. Specifically, during $24 \mathrm{~h}$ hypoxic exposures, RNA oxidative damage was reduced in brain and skeletal muscle by $44.17 \pm$ 12.53\% and $42.11 \pm 7.91 \%$, respectively, as compared with normoxic levels (Figure 2.3). RNA 
oxidative damage was also decreased in kidney, but this occurred after the 4 h hypoxic insult, where levels were reduced by $58.89 \pm 12.04 \%$, from normoxic values, making kidney the only tissue with a significant change in RNA damage after 4 h hypoxia (Figure 2.3). The only tissue to show elevated levels of RNA oxidative damage was heart, with a 1.71-fold increase after a $24 \mathrm{~h}$ hypoxic exposure (Figure 2.3). In contrast, RNA oxidative damage did not change in either liver or WAT during hypoxic exposures (Figure 2.3).

\subsection{4 $\quad$ All tissues except brain undergo lipid peroxidation during hypoxia}

TBARS levels are often used an indirect measurement of lipid peroxidation. While $4 \mathrm{~h}$ hypoxic brain was unaffected, TBARS levels in brain decreased by $54.59 \pm 4.89 \%$ during $24 \mathrm{~h}$ hypoxia exposure, relative to normoxic levels (Figure 2.4). Hypoxic liver displayed the largest increase in TBARS during both $4 \mathrm{~h}$ and $24 \mathrm{~h}$ hypoxia exposures, with 9.86 -fold and 5.09-fold increases, respectively (Figure 2.4). Muscle and kidney exhibited similar patterns, in which values after $4 \mathrm{~h}$ hypoxia did not significantly change; however, during $24 \mathrm{~h}$ hypoxia exposures, TBARS

levels were elevated by 1.52-fold and 1.86-fold, respectively (Figure 2.4). As for WAT, levels of TBARS increased by 2.48 -fold during the $4 \mathrm{~h}$ hypoxia exposure but remained unchanged during the 24 h hypoxic exposure (Figure 2.4). Naked mole-rat hearts did not cross-react with the TBARS assay. A possible explanation for this could be blood contamination, despite the heparin treatment that was performed prior to the TBARS assay to minimize such contamination.

\subsubsection{Brains and hearts display the most antioxidant defense capacity}

The comparison of each tissue's innate antioxidant capacity during acute $(4 \mathrm{~h})$ and chronic (24 h) hypoxia revealed that antioxidant capacity increases during hypoxia in naked mole-rat brain and heart (Figure 2.5). In brain, 4 hours of hypoxia did not have an effect, but 24 h hypoxia resulted in a 2.53-fold increase in antioxidant capacity, as compared to normoxic values (Figure 
2.5). In hearts, both acute and chronic hypoxia exposures led to significant increases in antioxidant capacity: 2.81-fold and 2.49-fold increases, respectively (Figure 2.5). The only tissue to exhibit a significant reduction in antioxidant capacity was liver $(22.55 \%$ decrease after $24 \mathrm{~h}$ hypoxia) (Figure 2.5). In contrast, antioxidant capacity was unchanged by hypoxia in skeletal muscle, kidney, and WAT, relative to normoxic controls (Figure 2.5).

\subsubsection{Dynamic tissue-specific regulation of DNA damage repair during hypoxia}

Relative phosphorylated levels of four proteins (phospho-Chk1, phospho-Chk2, phosphoH2A.X, and phospho-p53) and total levels of three proteins (ATR, MDM2, and p21) from the DNA damage repair pathway were examined under acute and chronic hypoxia in naked mole-rat tissues (Figure 2.6). Each tissue displayed a distinct expression profile, with liver being the only tissue where protein levels were unaffected by hypoxia (Figure 2.6C).

In brain, we found reduced levels of Chk2 during $4 \mathrm{~h}$ hypoxia and of MDM2 during $24 \mathrm{~h}$ hypoxia (Figure 2.6A). By contrast, levels of H2A.X in brain were upregulated after $4 \mathrm{~h}$ hypoxia and levels of p21 were upregulated after $24 \mathrm{~h}$ hypoxia (Figure 2.6A). All changes observed in both cardiac and skeletal muscle were hypoxia-downregulated (Figure 2.6B and 2.D). Levels of Chk1, MDM2, and p21 were downregulated in both $4 \mathrm{~h}$ and $24 \mathrm{~h}$ hypoxic hearts (Figure 2.6B). While in skeletal muscle, levels Chk1 and H2A.X were downregulated in both $4 \mathrm{~h}$ and $24 \mathrm{~h}$ hypoxic exposures (Figure 2.6D).

In kidneys, hypoxia-responsive changes were only observed during 4 h hypoxia exposure, where levels of ATR, Chk1, Chk2, H2A.X, MDM2, and p21 were upregulated, indicating that all but P53 were upregulated during $4 \mathrm{~h}$ exposures in kidneys (Figure 2.6E). In WAT, all seven proteins were significantly altered during hypoxia, and WAT was the only tissue in which a 
significant change in p53 occurred (a $56 \pm 3.9 \%$ decrease during $4 \mathrm{~h}$ hypoxia (Figure 2.6F)). WAT exhibited upregulated levels of ATR during $24 \mathrm{~h}$ hypoxia while the remaining changes were all hypoxia-induced downregulations (Figure 2.6F). Chk1, Chk2, p53, MDM2, and p21 were all downregulated during 4 h hypoxia exposures (Figure 2.6F). While only H2A.X and p21 were downregulated during 24 hypoxia exposures (Figure 2.6F).

\subsection{Discussion}

Naked mole-rats are inherently more resilient to oxidative damage as compared to mice, and older naked mole-rats are just as resistant as younger animals (Braude et al., 2021; Buffenstein et al., 2008) but, to date, the effects of low oxygen on naked mole-rat oxidative stress responses has not been probed. Herein we show that whereas levels of hypoxia-induced oxidative damage are tissue-specific, it appears that most tissues are resistant to hypoxia-induced oxidative damage. It is possible that the previously reported reduction in electron transport flux and mitochondrial $\mathrm{H}^{+}$ gradient in hypoxic naked mole-rats reduces aerobic metabolism, thereby limiting ROS production since mitochondria are major sources of oxygen radicals (Pamenter et al., 2018; Sanders and Greenamyren, 2013). This is combination with previous reports that naked mole-rat heart and

muscle have heightened ROS buffering systems (Munro et al., 2019). Before we discuss the main hypoxic trends observed in each tissue, oxidative damage accumulation, and repair system activation, it is important to first place our findings in the appropriate biological context. The oxidative damage measurements we report for each tissue represent the net levels of oxidative damage, or rather the 'steady-state' level of oxidized products. This means that each measurement of an oxidative stress marker is a result of several interacting processes associated with ROS 
generation, neutralization of ROS by antioxidants, damage repair, and removal of damaged products (Rodriguez et al., 2011).

An overall look at the hypoxia-induced damage accrued by naked mole-rats reveals that brain appears to be one of the more resilient tissues to hypoxic insults (Figures 2.1-2.5). Work on brains from hypoxic naked mole-rats have shown that they express many cell survival proteins as well as a subset of microRNAs that facilitate various neuroprotective roles (Hadj-Moussa et al., 2021; Hawkins et al., 2019; Logan et al., 2020; Nguyen et al., 2019). Conversely, the increased vulnerability to hypoxia measured in liver was in the form of elevated levels of TBARS, reduced antioxidant capacity, and minimal activation of the DNA repair pathway (Figure 2.1-2.6). Another global observation was that tissues displayed more significant changes in levels of oxidative damage proteins and antioxidant and repair mechanisms during the chronic $24 \mathrm{~h}$ hypoxic exposure than during the acute $4 \mathrm{~h}$ bouts of hypoxia. Below we discuss the hypoxia-induced response of each of the oxidative markers as well as the antioxidant neutralization and DNA repair system that combats oxidative stress in the hypometabolic state.

\subsubsection{No protein, little nucleic acid, and increased lipid oxidative damage during hypoxia}

\subsubsection{Protein carbonylation}

The most general marker of protein oxidation is protein carbonyl content, which measures the abundance of side-chain groups on amino acids that have been transformed into carbonyls. Despite proteins being prime targets for oxidative damage, the only marker that appeared to be impervious to hypoxia-induced oxidative damage in naked mole-rats was protein carbonylation, for which there is no change in any of the tissues after both $4 \mathrm{~h}$ and $24 \mathrm{~h}$ hypoxic exposures (Figure 2.1). This corroborates work performed on multiple mole-rat species with varying degrees of hypoxia tolerance, including naked mole-rats, in which protein carbonylation levels of whole brains are 
unchanged during $4 \mathrm{~h}$ hypoxic exposures (Logan et al., 2020). Naked mole-rats are known to have inherently elevated levels of protein carbonylation and these increased levels of carbonylation would be detrimental in most organisms, but do not appear to affect protein structure or function in mole-rats (Lewis et al., 2013). Indeed, analysis of oxidized naked mole-rat proteins suggests that they have structural resistance to misfolding, a lack of functional impairment, and a reduced sensitivity to form non-reducible oligomers and aggregates (De Waal et al., 2013; Pérez et al., 2009). As such, the naturally high levels of protein carbonylation may have masked any changes that would have typically been induced during hypoxia exposures.

\subsubsection{DNA oxidative damage}

Similarly, oxidation of DNA, which typically occurs at guanine bases, converting them into 8-Oxo-2'-deoxyguanosine (8-OHdG), are not increased by hypoxia in any tissues tested except kidney, in which DNA oxidative damage increases during both short and long hypoxic exposures (Figure 2.2). Why kidneys would be more susceptible to hypoxia-induced DNA oxidation is not known, particularly as kidneys display one of the more robust DNA damage repair responses, wherein phosphorylated levels of Chk1 and total protein levels of ATR, MDM2, and p21 are all elevated after $4 \mathrm{~h}$ hypoxic exposure (Figure 2.6E). In the following sections we discuss the DNA damage repair pathway in more detail, but it should be noted that this pathway is responsible for more than just the repair of oxidized DNA, as it is also activated for strand break repair, cell cycle regulation, and autophagy (Chatterjee and Walker, 2017; Gomes et al., 2017; Murray and Carr, 2018). It would be of interest for future research to investigate the enhanced vulnerability of kidney to DNA oxidation. Additionally, DNA oxidative damage is also inherently higher in naked molerat kidneys, as compared to mice (Andziak et al., 2006). 


\subsubsection{RNA oxidative damage}

While RNA is more abundant than DNA and accounts for approximately $80-90 \%$ of total cellular nucleic acids, there is a paucity of studies on RNA damage (Li et al., 2014). Recent work suggests not only that RNA is more vulnerable to ROS and RNS damage than its DNA counterpart, but that dysfunctional oxidized RNA is implicated in various diseases, including neurodegeneration, cancer, and metabolic diseases (Li et al., 2020, 2014; Wurtmann and Wolin, 2009). The common misconception that the rapid turnover and degradation of RNA should ameliorate the oxidization of RNA, is not accurate. While oxidation takes only minutes to occur, the half-life of mRNA is typically on the order of hours. Hence, there is still opportunity for deleterious outcomes ( $\mathrm{Li}$ et al., 2014). While the molecular mechanisms responsible for RNA oxidation remain largely unknown, as is the case for RNA modifications in general, the most abundant RNA oxidation marker is 8-hydroxyguanosine (8-OHG) (Li et al., 2006). This mutagenic modification is associated with strand breakage and base removal and is a by-product of hydroxyl radical attack (Castellani et al., 2008; Jacobs et al., 2010; Nunomura et al., 2009).

Curiously, when levels of RNA oxidative damage are examined in naked mole-rat tissues, levels decrease in $24 \mathrm{~h}$ hypoxic brain and muscle, and $4 \mathrm{~h}$ hypoxic kidney, whereas $24 \mathrm{~h}$ hypoxic hearts were the only tissue in which increased levels of RNA oxidative damage are observed (Figure 2.3). It should be noted that since we only measured levels of $8-\mathrm{OHG}$, actual levels of oxidized RNA are likely higher, as RNA can be oxidized in many forms (Li et al., 2014). Indeed, although natural degradation of oxidized RNA likely contributes to the lower levels of RNA oxidation in hypoxic brains, skeletal muscle, and kidney, the reduction rate of oxidized RNA is faster than what can be expected by normal RNA turnover, suggesting a selective mechanism that targets oxidized RNA for efficient degradation (Liu et al., 2012). A study on E. coli found that 
under oxidative stress conditions, selective degradation accounts for the elimination of most oxidized RNA and, unlike with DNA damage, most cells should be able to afford degradation of low amounts of oxidized RNA to maintain a functional RNA repertoire (Barregard et al., 2013; Li et al., 2014). As such, it is possible that the selective degradation of these aberrant oxidized RNA molecules during hypoxia occurs in naked mole-rat tissues with decreased levels of RNA oxidation. Currently, it is speculated that DNA and RNA share some of the same oxidative damage repair pathways, and that the RNA degradation enzymes involved in mRNA decay, degradation of stable RNAs, and RNA quality control are also involved, but this is still under investigation (Deutscher, 2006; Li et al., 2020; Mikolaskova et al., 2018).

\subsubsection{Lipid peroxidation}

Similar to the other oxidative damage markers examined, the level of lipid peroxidation products depends on both the duration and severity of hypoxia (Møller et al., 2008). Peroxidation results in lipid degeneration and cell membrane damage that alters membrane fluidity and the structure and function of membrane-associated proteins, potentially leading to apoptosis (Behn et al., 2007; Rauchová et al., 2012). Herein, lipid peroxidation was assessed by measuring malondialdehyde production, a naturally occurring product of lipid peroxidation, using the TBARS assay (Rael et al., 2004). TBARS levels increase in most tissues under at least one of the hypoxia exposures, except for brain, in which TBARS decreases during $24 \mathrm{~h}$ hypoxia, relative to normoxia

(Figure 2.4). While controlling lipid peroxidation allows hypoxia-tolerant animals to cope with increased ROS production more efficiently, studies on naked mole-rats demonstrate their ability to non-detrimentally accumulate large amounts of oxidative damage (Behn et al., 2007).

TBARS levels increase in $24 \mathrm{~h}$ hypoxic in skeletal muscle and kidney, and $4 \mathrm{~h}$ hypoxic WAT by 2.48-fold, and robust 9.9-fold and 5.1-fold in kidney during 4 and 24 h hypoxia (Figure 2.4). 
The large accumulation of TBARS in hypoxic liver could be attributed to the myriad of reactions that the liver, the body's metabolic hub, coordinates. Indeed, despite the depressed metabolism that naked mole-rats exhibit during hypoxia, low oxygen availability during these periods means that reducing equivalents accumulate in the respiratory chain, inevitably leading to ROS formation (Rauchová et al., 2012). Studies have shown that levels of lipid peroxidation are elevated in naked mole-rats as compared to mice, and that lipid damage levels do not change with age in naked molerats (Andziak et al., 2006). It is interesting to note that naked mole-rat alter the composition of their lipid membranes during hypoxia and that their membranes contain less easily oxidized n-3 polyunsaturated fatty acids (PUFA) and a higher proportion of more oxidation resistant n-6 PUFAs and monounsaturated fatty acids, providing a possible mechanism for mitigating accrued oxidative damage (Farhat et al., 2020; Hulbert et al., 2006).

The observed reduction of TBARS levels in hypoxic brain could act to protect this critical fatty tissue from damage despite brain being typically very susceptible to ROS damage and lipid peroxidation due to their high rate of oxidative metabolic activity, high concentrations of polyunsaturated fatty acids in membranes, presence of iron ions, and low antioxidant enzyme activities (Rauchová et al., 2012). However, this is not observed in hypoxic naked mole-rat brain, which appear to limit and reduce the accumulation of TBARS during hypoxia. Similar to the increase in liver lipid peroxidation levels, naked mole-rat brain accumulates higher levels of neuronal lipid peroxidation than mice (Edrey et al., 2014). Indeed, naked mole-rat brain from animals of all ages accumulate neuronal granules of various degradation products, suggestive of lipofuscinosis, caused by lipid peroxidation that results from cellular metabolism and accumulates with age (Ward et al., 2021). In young naked mole-rats, this is due to a low antioxidant potential, 
associated with low induction levels of Hsp70 and heme oxygenase, in addition to low ubiquitinproteosome activity (Edrey et al., 2014).

\subsubsection{Neutralization and repair}

Eliminating and neutralizing ROS generated as by-products of aerobic metabolism and from conditions that exacerbate oxidative stress, such as hypoxia, require a complex suite of mechanisms that work to limit and alleviate the potential for accruing oxidative damage.

\subsubsection{Antioxidant capacity}

Defense against free-radical-induced damage via coordinated enzymatic and non-enzymatic antioxidant molecules prevents auto-catalytic chain reactions that draw electrons from cellular macromolecules (Lewis et al., 2013; Rauchová et al., 2012). A look at the antioxidant capacity indicates that naked mole-rat brain and heart have increased antioxidant capacities during $24 \mathrm{~h}$ hypoxic exposures, with hearts also having significant protection during $4 \mathrm{~h}$ hypoxic exposures (Figure 2.5). Elevated antioxidant capacities are a common factor that predispose species for hypoxia and anoxia tolerance (Lutz and Milton, 2004). This enhanced level of protection in organs that are particularly sensitive to ROS damage during hypoxia suggests a well-developed strategy for protection against further increases in oxidative damage. It is possible that the increased antioxidant capacity observed in hypoxic brain and heart is part of the phenomenon known as "preparation for oxidative stress". This theory states that many animals that undergo seasonal hypoxic and hypometabolic episodes sometimes display elevated antioxidant potentials as a means of preemptively increasing their antioxidant defenses and protective mechanisms in anticipation for increased ROS production during hypoxia and reoxygenation (Giraud-Billoud et al., 2019). 
Despite the presence of multiple antioxidants within tissues and multiple back-up systems to compensate for depleted antioxidants, a cell's total antioxidant capacity can never fully prevent oxidative damage. Total antioxidant capacity in muscle, kidney, and WAT of mole-rats are unaffected by hypoxia, whereas there is a significant downregulation in liver after a $24 \mathrm{~h}$ hypoxic exposure (Figure 2.5). It is possible that the liver's role as the body's metabolic hub could explain the observed reduced antioxidant capacity. Future work could more deeply characterize key antioxidant enzyme activities and glutathione levels during hypoxic exposures. Interestingly, a low GSH/GSSG ratio in naked mole-rats, as compared to mice, indicated a poor antioxidant capacity, since the ratio of reduced GSH to glutathione disulfide is considered a reliable indicator of cellular oxidative stress (Andziak et al., 2006). This is in contrast to GSH-dependency for enhanced mitochondrial ROS scavenging capacity in naked mole-rats, relative to mice (Munro et al., 2019).

\subsubsection{DNA damage repair pathway}

Although significant DNA oxidative damage is only detected in hypoxic kidneys (Figure 2.2), we decided to investigate the central DNA repair proteins in all tissues to determine if tissues that were resistant to oxidative DNA damage have stronger repair systems, and vice versa (Figure 2.6). The accumulation of unrepaired damage that results from endogenous ROS formation and other mutagenic stressors triggers the DNA damage response and repair systems (Santos et al., 2018). Many DNA repair mechanisms including base excision repair, mismatch repair, nucleotide excision repair, and double-strand-break repair, are constitutively expressed to maintain and protect cells (Ciccia and Elledge, 2010; Lindahl and Barnes, 2000).

The DNA damage response is a complex and highly choreographed signal transduction pathway. Briefly, the activation of the phosphatidylinositol-3-kinase-like (PI3K) kinases, ATR and ATM, act as key sensors of DNA damage. This leads to the phosphorylation of checkpoint kinases 
(Chk1 at Ser345 and Chk2 at Thr68) and H2A.X at Ser139, a hallmark of DNA damage that opens chromatin for repair complex formation (Cazzalini et al., 2010). This phosphorylation has been reported in hypoxic conditions as a means to halt the cell cycle and allow the repair of DNA damaged by ROS. Under hypoxia, ATR also induces the phosphorylation of p53 at Ser15 and this is critical for releasing p53 from its negative regulator MDM2 and for facilitating p53 nuclear retention (Shinozaki et al., 2003). Subsequently, p53 is then responsible for orchestrating cell cycle arrest, apoptosis, and the induction of downstream DNA repair proteins (Hammond et al., 2003; Sun et al., 2017).

A look at the DNA repair response of hypoxic kidney, the only tissue to display an increase in DNA oxidative damage, revealed an increase in DNA repair proteins, possibly to mitigate hypoxia-induced DNA oxidation (Figure 2.2 and 2.6E). While no changes in repair proteins are observed in $24 \mathrm{~h}$ hypoxic kidneys, total protein levels of ATR, MDM2, and p21 are upregulated, along with an increase in phosphorylated Chk1 (Ser345) during the shorter $4 \mathrm{~h}$ hypoxic episode (Figure 2.6E). Although ATM and ATR perform redundant functions, they also respond to specific types of damage, where the phosphorylation of ATM-Chk2 is associated with double strand breaks and ATR-Chk1 mediates single and double strand breaks and various other types (Sun et al., 2017). The induction of the ATR-Chk1 axis in hypoxic kidneys suggests the activation of DNA single-stranded break repair during hypoxia (Smith et al., 2010). Increased levels of MDM2, a negative regulator of $\mathrm{p} 53$, suggest the suppression of downstream p53 transcriptional activity. The role for hypoxia-elevated p21 proteins is likely also linked to cell cycle controls, as p21 is a principal mediator of the cell cycle that is rapidly recruited to the site of DNA damage and responds by inactivating cyclins and CDK complexes, as well as through the inhibition of DNA replication and transcription, and the regulation of apoptosis (Perucca et al., 2006). It is intriguing 
that levels of all the DNA response proteins that are activated during hypoxia return to normoxic levels during chronic hypoxia, a possible explanation for this attenuated response during prolonged hypoxia could be to conserve energy, given that naked mole-rats are well adapted to overcome elevated levels of oxidative DNA damage. A finding that is consistent with a study that demonstrated that ATP-independent heat shock protein levels were maintained during hypoxia while ATP-dependant chaperones were downregulated (Nguyen et al., 2019).

In the other tissues examined, most hypoxia-induced proteins are reduced, either through reductions in expression or phosphoprotein content (Figure 2.6). Globally, this could be a way for naked mole-rats to conserve energy as they do not need to actively maintain DNA damage responses during hypoxia. For example, in naked mole-rat brain, the downregulation of ATR (after $24 \mathrm{~h}$ of hypoxia) and reduced phosphorylation of Chk2 illustrate the lack of activation of the DNA damage response signal transduction pathway, which contrasts with the increased abundance of phospho-H2A.X (after $4 \mathrm{~h}$ of hypoxia) (Figure 6A). Curiously, despite both liver and WAT exhibiting no oxidative damage, liver DNA repair proteins are largely unaffected by hypoxia, with only p21 downregulated during $24 \mathrm{~h}$ hypoxic exposure. In contrast, WAT has the most dynamic response, with most of the proteins and phosphorylated forms examined being downregulated, except for increased levels of total ATR protein during $24 \mathrm{~h}$ hypoxia, suggesting a reduced capacity for DNA damage repair in hypoxic adipose tissue (Figures 2.2, 2.6A, and 2.6F).

Although this was not the focus of our study, it should be noted that many of the proteins involved in DNA damage repair are also regulators of the cell cycle. The cell cycle is controlled by many inputs including both DNA damage and hypoxia, and many animals that employ metabolic rate depression strategies rely on cell cycle arrest as a mechanism by which to limit unnecessary energy expenditure (Biggar and Storey, 2012; Wu and Storey, 2012; Zhang and 
Storey, 2012). It is possible that the changes to cell cycle regulators seen in this study could be coordinating an energy-saving mechanism that regulates/suppresses cell cycle activity during hypoxia-induced hypometabolic exposures.

\subsection{Conclusion}

This multi-tissue examination of oxidative damage markers revealed that naked mole-rats do not accrue drastically elevated levels of oxidized macromolecules during hypoxia and that most tissues were only susceptible to minimal damage. For example, no tissue displayed evidence of hypoxia-induced changes to protein carbonylation levels. This could be because naked mole-rats have inherently higher levels of oxidative damage when compared to mice, and that these levels remain constant regardless of the oxidation levels in their environments. Alternatively, it could be due to enhanced buffering capacities of certain tissues. Each tissue displayed a unique damage fingerprint, where brain and heart exhibited a strong antioxidant capacity, liver exhibited elevated lipid peroxidation, kidneys had high levels of DNA damage, and WAT had a reduced activation of the DNA damage and repair pathway. Taken together, these findings suggest that naked molerats appear to be largely unaffected by hypoxia-induced oxidative damage. 


\subsection{References}

Al-attar, R., Childers, C.L., Nguyen, V.C., Pamenter, M.E., Storey, K.B., 2020. Differential protein phosphorylation is responsible for hypoxia-induced regulation of the Akt/mTOR pathway in naked mole-rats. Comp. Biochem. Physiol. Part A Mol. Integr. Physiol. 242, 110653.

Andziak, B., O’Connor, T.P., Qi, W., Dewaal, E.M., Pierce, A., Chaudhuri, A.R., Van Remmen, H., Buffenstein, R., 2006. High oxidative damage levels in the longest-living rodent, the naked mole-rat. Aging Cell 5, 463-471.

Barregard, L., Møller, P., Henriksen, T., Mistry, V., Koppen, G., Rossner, P., Sram, R.J., Weimann, A., Poulsen, H.E., Nataf, R., Andreoli, R., Manini, P., Marczylo, T., Lam, P., Evans, M.D., Kasai, H., Kawai, K., Li, Y.S., Sakai, K., Singh, R., Teichert, F., Farmer, P.B., Rozalski, R., Gackowski, D., Siomek, A., Saez, G.T., Cerda, C., Broberg, K., Lindh, C., Hossain, M.B., Haghdoost, S., Hu, C.W., Chao, M.R., Wu, K.Y., Orhan, H., Senduran, N., Smith, R.J., Santella, R.M., Su, Y., Cortez, C., Yeh, S., Olinski, R., Loft, S., Cooke, M.S., 2013. Human and methodological sources of variability in the measurement of urinary 8-oxo7,8-dihydro-2'-deoxyguanosine. Antioxidants Redox Signal. 18, 2377-2391.

Behn, C., Araneda, O.F., Llanos, A.J., Celedón, G., González, G., 2007. Hypoxia-related lipid peroxidation: Evidences, implications and approaches. Respir. Physiol. Neurobiol. 158, 143150.

Biggar, K.K., Storey, K.B., 2012. Evidence for cell cycle suppression and microRNA regulation of cyclin D1 during anoxia exposure in turtles. Cell Cycle 11, 1705-1713.

Braude, S., Holtze, S., Begall, S., Brenmoehl, J., Burda, H., Dammann, P., del Marmol, D., Gorshkova, E., Henning, Y., Hoeflich, A., Höhn, A., Jung, T., Hamo, D., Sahm, A., Shebzukhov, Y., Šumbera, R., Miwa, S., Vyssokikh, M.Y., von Zglinicki, T., Averina, O., Hildebrandt, T.B., 2021. Surprisingly long survival of premature conclusions about naked mole-rat biology. Biol. Rev. 96, 376-393.

Browe, B.M., Vice, E.N., Park, T.J., 2020. Naked Mole-Rats: Blind, Naked, and Feeling No Pain. Anat. Rec. 303, 77-88.

Buffenstein, R., 2005. The naked mole-rat: A new long-living model for human aging research. Journals Gerontol. - Ser. A Biol. Sci. Med. Sci.

Buffenstein, R., Edrey, Y.H., Yang, T., Mele, J., 2008. The oxidative stress theory of aging: Embattled or invincible? Insights from non-traditional model organisms. Age (Omaha).

Castellani, R.J., Nunomura, A., Rolston, R.K., Moreira, P.I., Takeda, A., Perry, G., Smith, M.A., 2008. Sublethal RNA oxidation as a mechanism for neurodegenerative disease. Int. J. Mol. Sci.

Cazzalini, O., Scovassi, A.I., Savio, M., Stivala, L.A., Prosperi, E., 2010. Multiple roles of the cell cycle inhibitor p21CDKN1A in the DNA damage response. Mutat. Res. - Rev. Mutat. Res. 
Chatterjee, N., Walker, G.C., 2017. Mechanisms of DNA damage, repair, and mutagenesis. Environ. Mol. Mutagen.

Chung, D., Dzal, Y.A., Seow, A., Milsom, W.K., Pamenter, M.E., 2016. Naked mole-rats exhibit metabolic but not ventilatory plasticity following chronic sustained hypoxia. Proc. R. Soc. B Biol. Sci. 283, 20160216.

Ciccia, A., Elledge, S.J., 2010. The DNA Damage Response: Making It Safe to Play with Knives. Mol. Cell.

De Waal, E.M., Liang, H., Pierce, A., Hamilton, R.T., Buffenstein, R., Chaudhuri, A.R., 2013. Elevated protein carbonylation and oxidative stress do not affect protein structure and function in the long-living naked-mole-rat: A proteomic approach. Biochem. Biophys. Res. Commun. 434, 815-819.

Deutscher, M.P., 2006. Degradation of RNA in bacteria: Comparison of mRNA and stable RNA. Nucleic Acids Res. 34, 659-666.

Edrey, Y.H., Hanes, M., Pinto, M., Mele, J., Buffenstein, R., 2011. Successful aging and sustained good health in the naked mole-rat: a long-lived mammalian model for biogerontology and biomedical research. ILAR J. 52, 41-53.

Edrey, Y.H., Oddo, S., Cornelius, C., Caccamo, A., Calabrese, V., Buffenstein, R., 2014. Oxidative damage and amyloid- $\beta$ metabolism in brain regions of the longest-lived rodents. J. Neurosci. Res. 92, 195-205.

Farhat, E., Devereaux, M.E.M., Pamenter, M.E., Weber, J.M., 2020. Naked mole-rats suppress energy metabolism and modulate membrane cholesterol in chronic hypoxia. Am. J. Physiol. - Regul. Integr. Comp. Physiol. 319, R148-R155.

Giraud-Billoud, M., Rivera-Ingraham, G.A., Moreira, D.C., Burmester, T., Castro-Vazquez, A., Carvajalino-Fernández, J.M., Dafre, A., Niu, C., Tremblay, N., Paital, B., Rosa, R., Storey, J.M., Vega, I.A., Zhang, W., Yepiz-Plascencia, G., Zenteno-Savin, T., Storey, K.B., HermesLima, M., 2019. Twenty years of the 'Preparation for Oxidative Stress' (POS) theory: Ecophysiological advantages and molecular strategies. Comp. Biochem. Physiol. Part A Mol. Integr. Physiol. 234, 36-49.

Gomes, L.R., Menck, C.F.M., Leandro, G.S., 2017. Autophagy roles in the modulation of DNA repair pathways. Int. J. Mol. Sci.

Hadj-Moussa, H., Zhang, J., Pifferi, F., Perret, M., Storey, K.B., 2020. Profiling torpor-responsive microRNAs in muscles of the hibernating primate Microcebus murinus. Biochim. Biophys. Acta - Gene Regul. Mech. 1863, 194473.

Hadj-Moussa, H., Pamenter, M.E., Storey, K.B., 2021. Hypoxic naked mole-rat brains use microRNA to coordinate hypometabolic fuels and neuroprotective defenses. J. Cell. Physiol. 236, 5080-5097. 
Hammond, E.M., Dorie, M.J., Giaccia, A.J., 2003. ATR/ATM targets are phosphorylated by ATR in response to hypoxia and ATM in response to reoxygenation. J. Biol. Chem. 278, 1220712213.

Hawkins, L.J., Hadj-Moussa, H., Nguyen, V.C., Pamenter, M.E., Storey, K.B., 2019. Naked molerats activate neuroprotective proteins during hypoxia. J. Exp. Zool. Part A Ecol. Integr. Physiol. 331, 571-576.

Hochachka, P.W., Buck, L.T., Doll, C.J., Land, S.C., 1996. Unifying theory of hypoxia tolerance: molecular/metabolic defense and rescue mechanisms for surviving oxygen lack. Proc. Natl. Acad. Sci. U. S. A. 93, 9493-8.

Holtze, S., Braude, S., Lemma, A., Koch, R., Morhart, M., Szafranski, K., Platzer, M., Alemayehu, F., Goeritz, F., Hildebrandt, T.B., 2018. The microenvironment of naked mole-rat burrows in East Africa. Afr. J. Ecol. 56, 279-289.

Houlahan, C.R., Kirby, A.M., Dzal, Y.A., Fairman, G.D., Pamenter, M.E., 2018. Divergent behavioural responses to acute hypoxia between individuals and groups of naked mole-rats. Comp. Biochem. Physiol. Part B Biochem. Mol. Biol. 224, 38-44.

Hulbert, A.J., Faulks, S.C., Buffenstein, R., 2006. Oxidation-Resistant Membrane Phospholipids Can Explain Longevity Differences Among the Longest-Living Rodents and Similarly-Sized Mice. Journals Gerontol. Ser. A Biol. Sci. Med. Sci. 61, 1009-1018.

Jacobs, A.C., Resendlz, M.J.E., Greenberg, M.M., 2010. Direct strand scission from a nucleobase radical in RNA. J. Am. Chem. Soc. 132, 3668-3669.

Krivoruchko, A., Storey, K.B., 2010. Forever young: mechanisms of natural anoxia tolerance and potential links to longevity. Oxid. Med. Cell. Longev. 3, 186-98.

Lewis, K.N., Andziak, B., Yang, T., Buffenstein, R., 2013. The naked mole-rat response to oxidative stress: Just deal with it. Antioxidants Redox Signal. 19, 1388-1399.

Li, Z., Chen, X., Liu, Z., Ye, W., Li, L., Qian, L., Ding, H., Li, P., Aung, L.H.H., 2020. Recent Advances: Molecular Mechanism of RNA Oxidation and Its Role in Various Diseases. Front. Mol. Biosci. 7, 184.

Li, Z., Malla, S., Shin, B., Li, J.M., 2014. Battle against RNA oxidation: Molecular mechanisms for reducing oxidized RNA to protect cells. Wiley Interdiscip. Rev. RNA.

Li, Z., Wu, J., DeLeo, C.J., 2006. RNA damage and surveillance under oxidative stress. IUBMB Life.

Lindahl, T., Barnes, D.E., 2000. Repair of endogenous DNA damage, in: Cold Spring Harbor Symposia on Quantitative Biology. Cold Spring Harbor Laboratory Press, pp. 127-133.

Liu, M., Gong, X., Alluri, R.K., Wu, J., Sablo, T., Li, Z., 2012. Characterization of RNA damage under oxidative stress in Escherichia coli. Biol. Chem. 393, 123-132. 
Logan, S.M., Szereszewski, K.E., Bennet, N.C., Hart, D.W., van Jaarsveld, B., Pamenter, M.E., Storey, K.B., 2020. The brains of six African mole-rat species show divergent responses to hypoxia. J. Exp. Biol.

Lutz, P.L., Milton, S.L., 2004. Negotiating brain anoxia survival in the turtle. J. Exp. Biol.

Mikolaskova, B., Jurcik, M., Cipakova, I., Kretova, M., Chovanec, M., Cipak, L., 2018. Maintenance of genome stability: the unifying role of interconnections between the DNA damage response and RNA-processing pathways. Curr. Genet.

Møller, P., Risom, L., Lundby, C., Mikkelsen, L., Loft, S., 2008. Hypoxia and oxidation levels of DNA and lipids in humans and animal experimental models. IUBMB Life 60, 707-723.

Munro, D., Baldy, C., Pamenter, M.E., Treberg, J.R., 2019. The exceptional longevity of the naked mole-rat may be explained by mitochondrial antioxidant defenses. Aging Cell 18, e12916.

Murray, J.M., Carr, A.M., 2018. Integrating DNA damage repair with the cell cycle. Curr. Opin. Cell Biol.

Nguyen, V.C., Deck, C.A., Pamenter, M.E., 2019. Naked mole-rats reduce the expression of ATPdependent but not ATP-independent heat shock proteins in acute hypoxia. J. Exp. Biol. 222.

Nunomura, A., Hofer, T., Moreira, P.I., Castellani, R.J., Smith, M.A., Perry, G., 2009. RNA oxidation in Alzheimer disease and related neurodegenerative disorders. Acta Neuropathol.

Orr, M.E., Garbarino, V.R., Salinas, A., Buffenstein, R., 2015. Sustained high levels of neuroprotective, high molecular weight, phosphorylated tau in the longest-lived rodent. Neurobiol. Aging 36, 1496-1504.

Pamenter, M.E., Dzal, Y.A., Thompson, W.A., Milsom, W.K., 2019. Do naked mole-rats accumulate a metabolic acidosis or an oxygen debt in severe hypoxia? J. Exp. Biol. 222, jeb.191197.

Pamenter, M.E., Lau, G.Y., Richards, J.G., Milsom, W.K., 2018. Naked mole-rat brain mitochondria electron transport system flux and $\mathrm{H}+$ leak are reduced during acute hypoxia. J. Exp. Biol. 221.

Park, T.J., Reznick, J., Peterson, B.L., Blass, G., Omerbašić, D., Bennett, N.C., Kuich, P.H.J.L., Zasada, C., Browe, B.M., Hamann, W., Applegate, D.T., Radke, M.H., Kosten, T., Lutermann, H., Gavaghan, V., Eigenbrod, O., Bégay, V., Amoroso, V.G., Govind, V., Minshall, R.D., Smith, E.S.J., Larson, J., Gotthardt, M., Kempa, S., Lewin, G.R., 2017. Fructose-driven glycolysis supports anoxia resistance in the naked mole-rat. Science 356, $307-311$.

Pérez, V.I., Buffenstein, R., Masamsetti, V., Leonard, S., Salmon, A.B., Mele, J., Andziak, B., Yang, T., Edrey, Y., Friguet, B., Ward, W., Richardson, A., Chaudhuri, A., 2009. Protein stability and resistance to oxidative stress are determinants of longevity in the longest-living rodent, the naked mole-rat. Proc. Natl. Acad. Sci. U. S. A. 106, 3059-3064. 
Perucca, P., Cazzalini, O., Mortusewicz, O., Necchi, D., Savio, M., Nardo, T., Stivala, L.A., Leonhardt, H., Cardoso, M.C., Prosperi, E., 2006. Spatiotemporal dynamics of p21CDKN1A protein recruitment to DNA-damage sites and interaction with proliferating cell nuclear antigen. J. Cell Sci. 119, 1517-1527.

Rael, L.T., Thomas, G.W., Craun, M.L., Curtis, C.G., Bar-Or, R., Bar-Or, D., 2004. Lipid peroxidation and the thiobarbituric acid assay: Standardization of the assay when using saturated and unsaturated fatty acids. J. Biochem. Mol. Biol. 37, 749-752.

Rauchová, H., Vokurková, M., Koudelová, J., 2012. Hypoxia-induced lipid peroxidation in the brain during postnatal ontogenesis. Physiol. Res.

Rodriguez, K.A., Wywial, E., Perez, V.I., Lambert, A.J., Edrey, Y.H., Lewis, K.N., Grimes, K., Lindsey, M.L., Brand, M.D., Buffenstein, R., 2011. Walking the Oxidative Stress Tightrope: A Perspective from the Naked Mole-Rat, the Longest-Living Rodent. Curr. Pharm. Des. 17, 2290-2307.

Saldmann, F., Viltard, M., Leroy, C., Friedlander, G., 2019. The naked mole-rat: A unique example of positive oxidative stress. Oxid. Med. Cell. Longev. 2019.

Sanders, L.H., Greenamyren, J.T., 2013. Oxidative damage to macromolecules in human Parkinson disease and the rotenone model. Free Radic. Biol. Med.

Santos, A.L., Sinha, S., Lindner, A.B., 2018. The good, the bad, and the ugly of ROS: New insights on aging and aging-related diseases from eukaryotic and prokaryotic model organisms. Oxid. Med. Cell. Longev.

Shepard, A., Kissil, J.L., 2020. The use of non-traditional models in the study of cancer resistance - the case of the naked mole-rat. Oncogene.

Shinozaki, T., Nota, A., Taya, Y., Okamoto, K., 2003. Functional role of Mdm2 phosphorylation by ATR in attenuation of p53 nuclear export. Oncogene 22, 8870-8880.

Smith, E.S.J., Schuhmacher, L.-N., Husson, Z., 2015. The naked mole-rat as an animal model in biomedical research: current perspectives. Open Access Anim. Physiol. 7, 137.

Smith, J., Mun Tho, L., Xu, N., A. Gillespie, D., 2010. The ATM-Chk2 and ATR-Chk1 pathways in DNA damage signaling and cancer, in: Advances in Cancer Research. Academic Press Inc., pp. 73-112.

Sun, B., Ross, S.M., Rowley, S., Adeleye, Y., Clewell, R.A., 2017. Contribution of ATM and ATR kinase pathways to p53-mediated response in etoposide and methyl methanesulfonate induced DNA damage. Environ. Mol. Mutagen. 58, 72-83.

Wang, T.H., Eaton, L., Pamenter, M.E., 2020. Nitric oxide homeostasis is maintained during acute in vitro hypoxia and following reoxygenation in naked mole-rat but not mouse cortical neurons. Comp. Biochem. Physiol. -Part A Mol. Integr. Physiol. 250. 
Ward, J.M., Cartoceti, A.N., Delaney, M.A., 2021. Brain Lesions in Aging Zoo-Housed Naked Mole-Rats (Heterocephalus glaber). Vet. Pathol. 58, 142-146.

Wu, C.W., Storey, K.B., 2012. Pattern of cellular quiescence over the hibernation cycle in liver of thirteen-lined ground squirrels. Cell Cycle 11, 1714-1726.

Wurtmann, E.J., Wolin, S.L., 2009. RNA under attack: Cellular handling of RNA damage RNA under attack: Cellular handling of RNA damage E. J. Wurtmann et.al. Crit. Rev. Biochem. Mol. Biol.

Xiao, B., Li, L., Xu, C., Zhao, S., Lin, L., Cheng, J., Yang, W., Cong, W., Kan, G., Cui, S., 2017a. Transcriptome sequencing of the naked mole-rat (Heterocephalus glaber) and identification of hypoxia tolerance genes. Biol. Open 6, 1904-1912.

Xiao, B., Wang, S., Yang, G., Sun, X., Zhao, S., Lin, L., Cheng, J., Yang, W., Cong, W., Sun, W., Kan, G., Cui, S., 2017b. HIF-1a contributes to hypoxia adaptation of the naked mole-rat. Oncotarget 8, 109941-109951.

Zhang, J., Storey, K.B., 2016. RBioplot: an easy-to-use R pipeline for automated statistical analysis and data visualization in molecular biology and biochemistry. PeerJ 4, e2436.

Zhang, J., Storey, K.B., 2012. Cell cycle regulation in the freeze-tolerant wood frog, Rana sylvatica. Cell Cycle 11, 1727-1742.

Zuo, W., Tang, X., Hou, C., 2020. Why Naked Mole-Rats Have High Oxidative Damage but Live a Long Life: A Simple Explanation Based on the Oxidative Stress Theory of Aging. Adv. Geriatr. Med. Res. 1-18. 


\section{$2.8 \quad$ Figures}

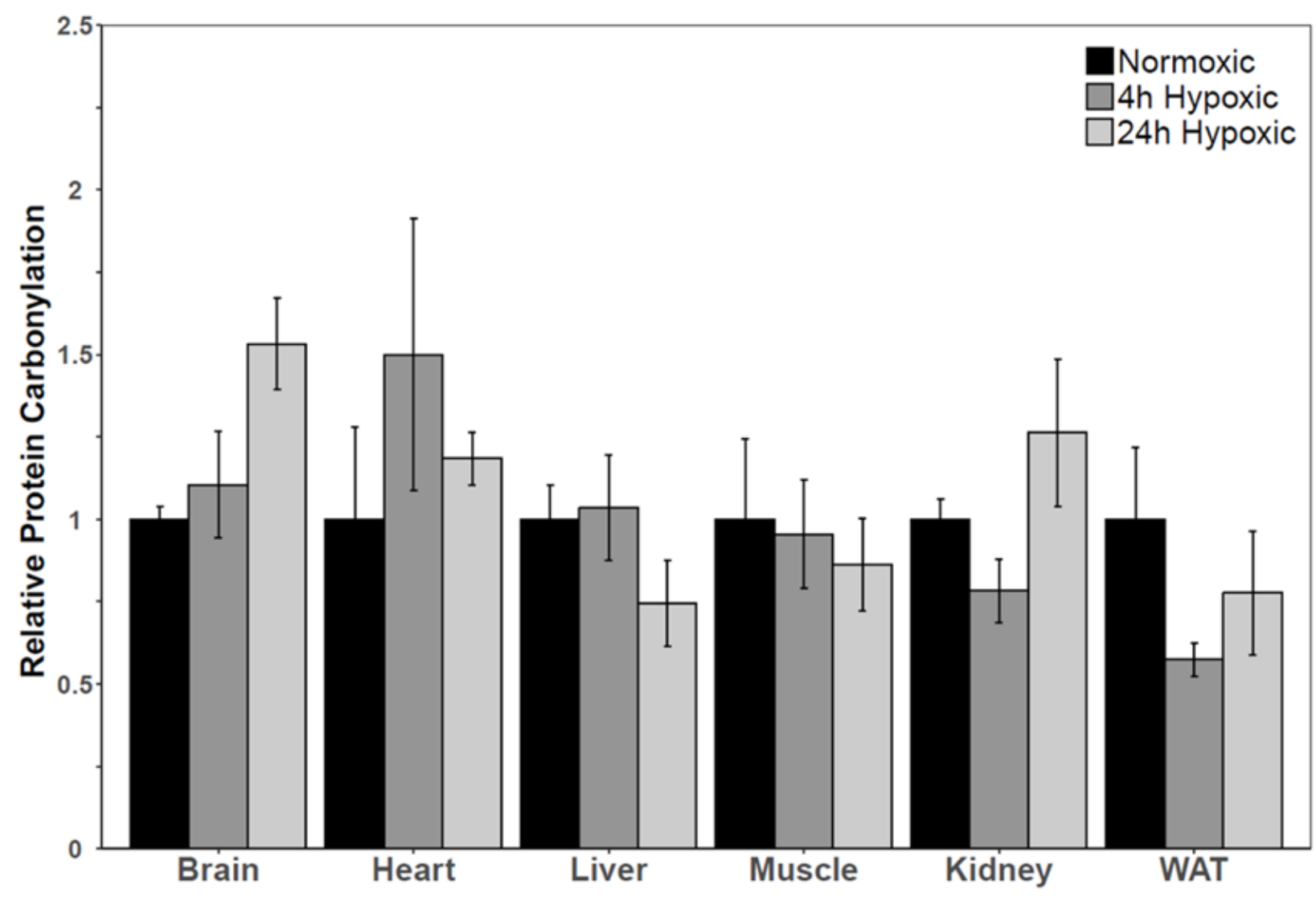

Figure 2.1. Protein carbonylation levels in hypoxic naked mole-rat tissues. Histogram comparing relative protein carbonylation $(\mathrm{nmol} / \mathrm{mL})$ in normoxic (control), $4 \mathrm{~h}$ hypoxic, and $24 \mathrm{~h}$ hypoxic naked mole-rat tissues (brain, heart, liver, skeletal muscle, kidney, WAT). Data are means \pm SEM ( $n=4$ independent biological replicates from different animals), with normoxic mean values standardized to 1 . Statistical analysis was preformed for each tissue using a one-way ANOVA with a Dunnett's post-hoc test $(p \leq 0.05)$ and significant changes are indicated with an asterisk. 


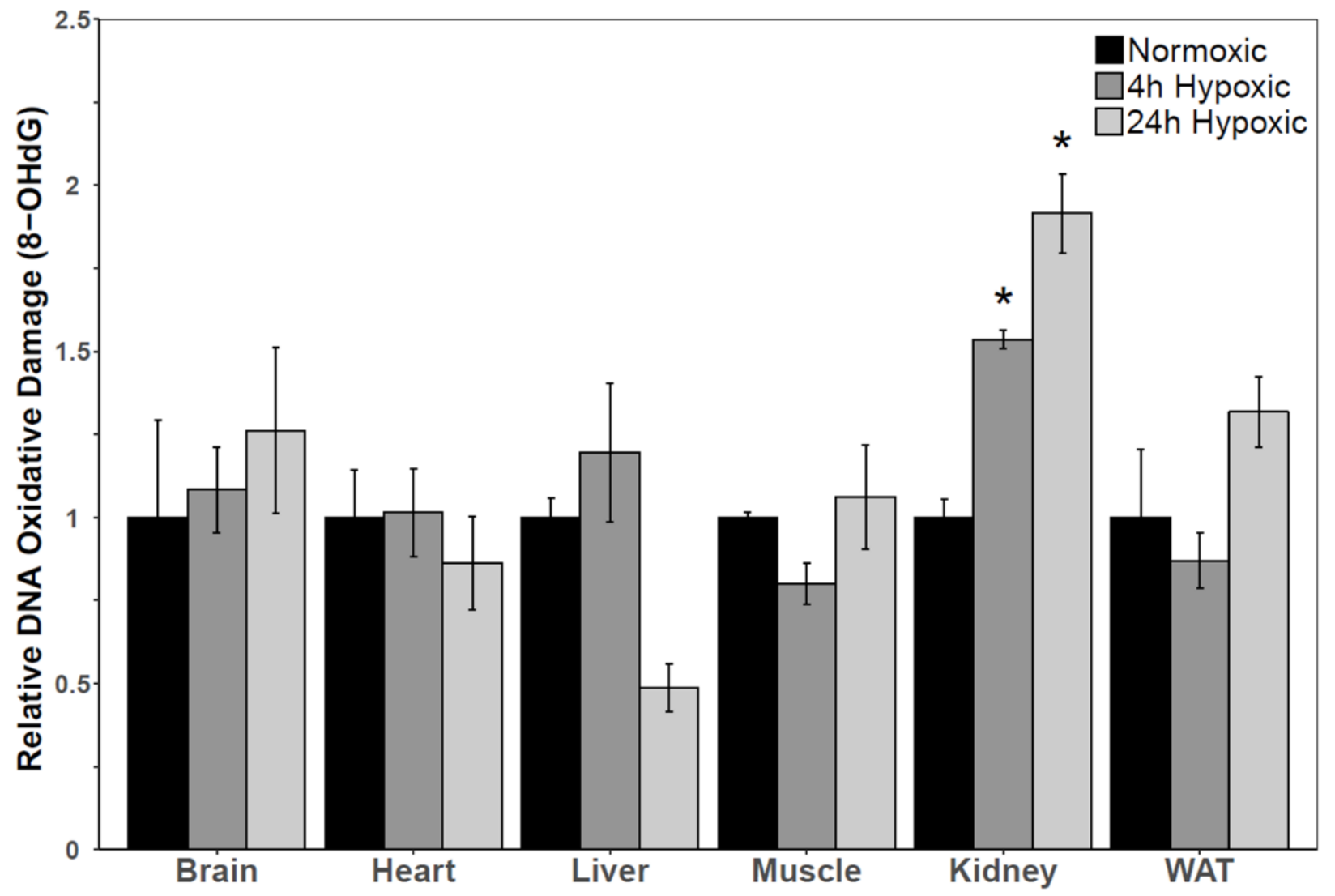

Figure 2.2. DNA oxidative damage levels in hypoxic naked mole-rat tissues. Histogram comparing relative DNA oxidative damage levels of $4 \mathrm{~h}$ hypoxic and $24 \mathrm{~h}$ hypoxic exposures, relative to normoxic (control) in six naked mole-rat tissues (brain, heart, liver, muscle, kidney, WAT). Other information as per Figure 2.1. 


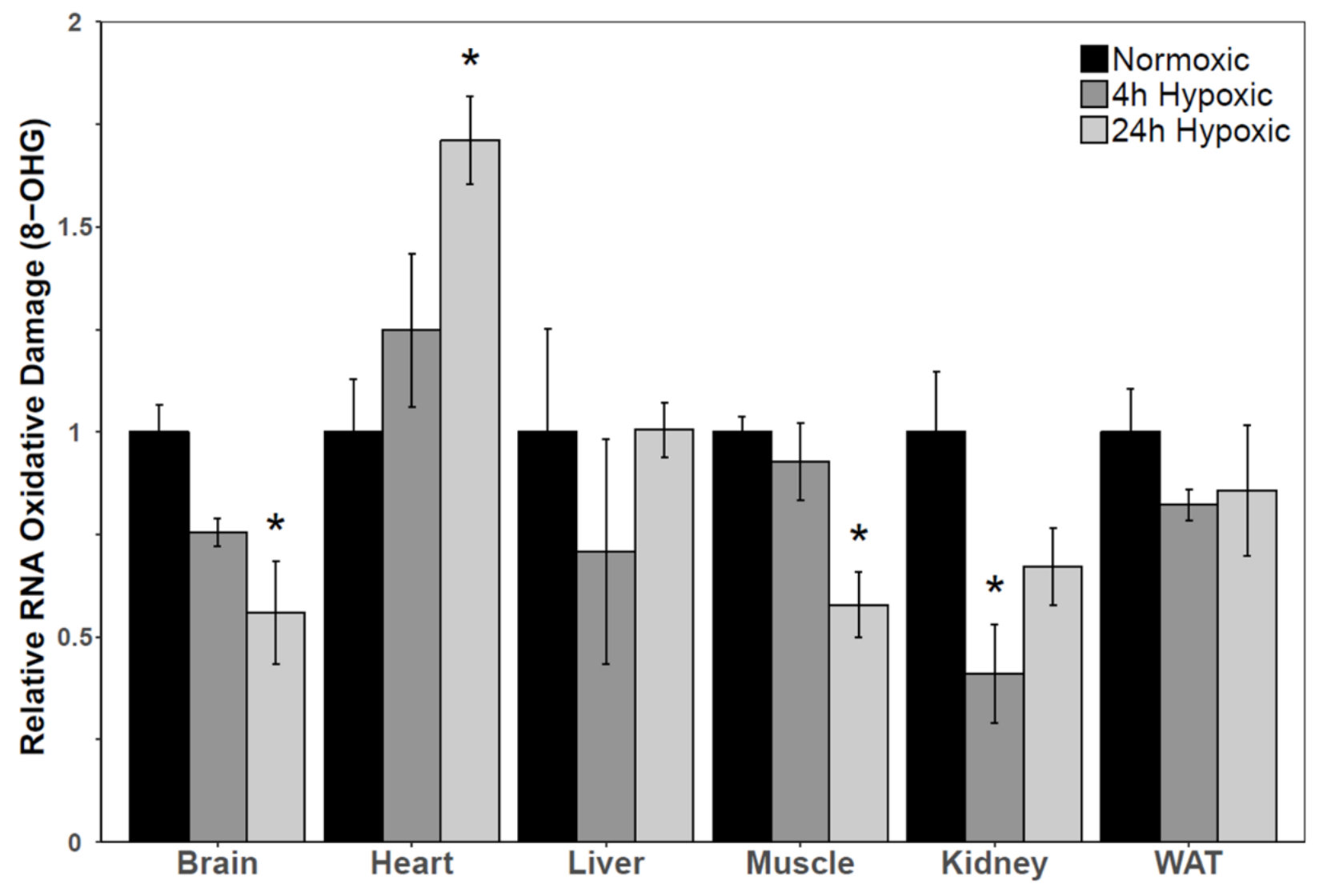

Figure 2.3. Relative RNA oxidative damage in hypoxic naked mole-rat tissues. Histogram comparing relative RNA oxidative damage levels of $4 \mathrm{~h}$ hypoxic and $24 \mathrm{~h}$ hypoxic exposures, relative to normoxic (control) in six naked mole-rat tissues (brain, heart, liver, muscle, kidney, WAT). Other information as per Figure 2.1. 


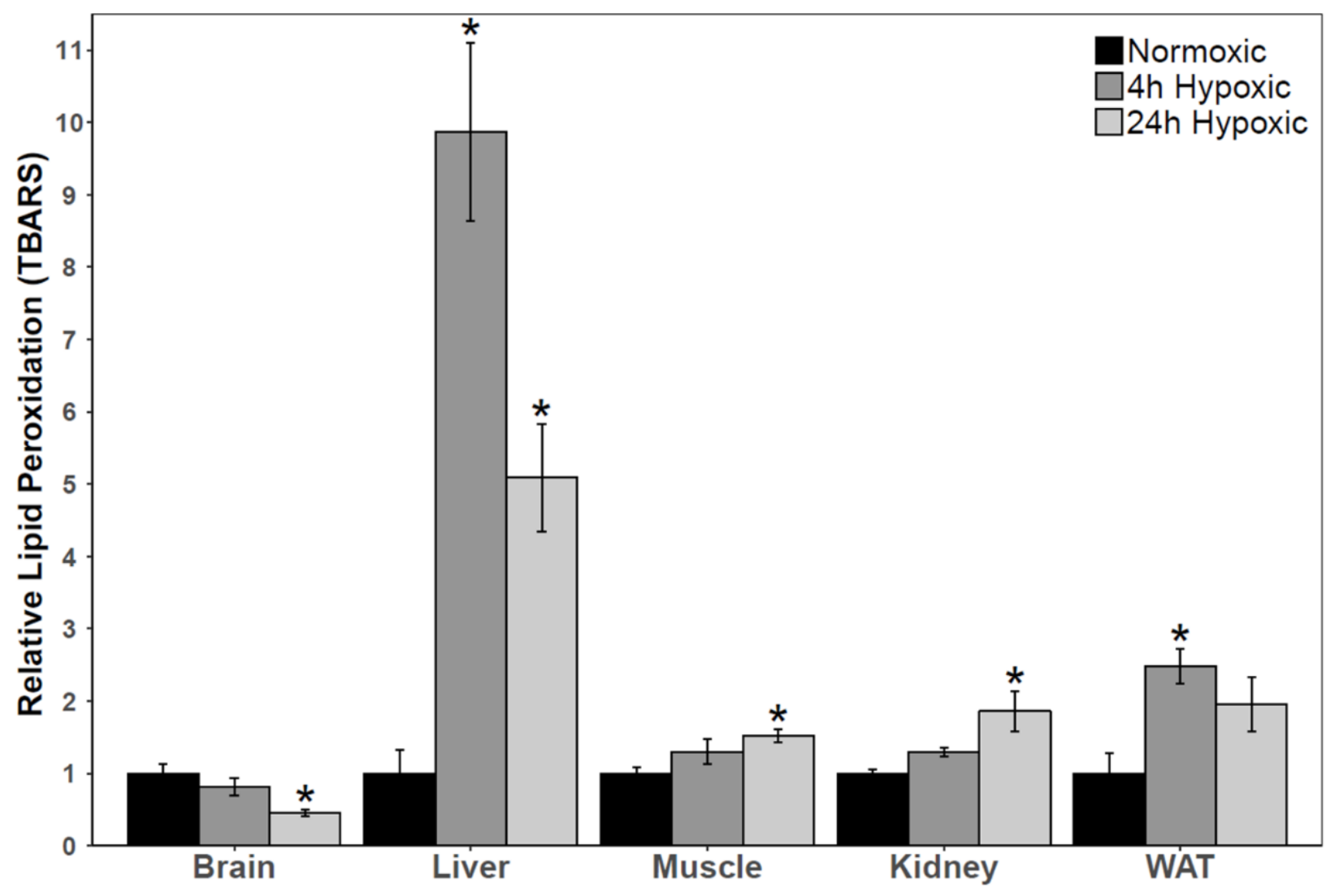

Figure 2.4. Lipid peroxidation as measured by TBARS concentrations in hypoxic naked mole-rat tissues. Histogram comparing relative TBARS concentration $(\mu \mathrm{M})$ of normoxic (control), $4 \mathrm{~h}$ hypoxic, and $24 \mathrm{~h}$ hypoxic naked mole-rat tissues (brain, liver, muscle, kidney, WAT). Other information as per Figure 2.1. 


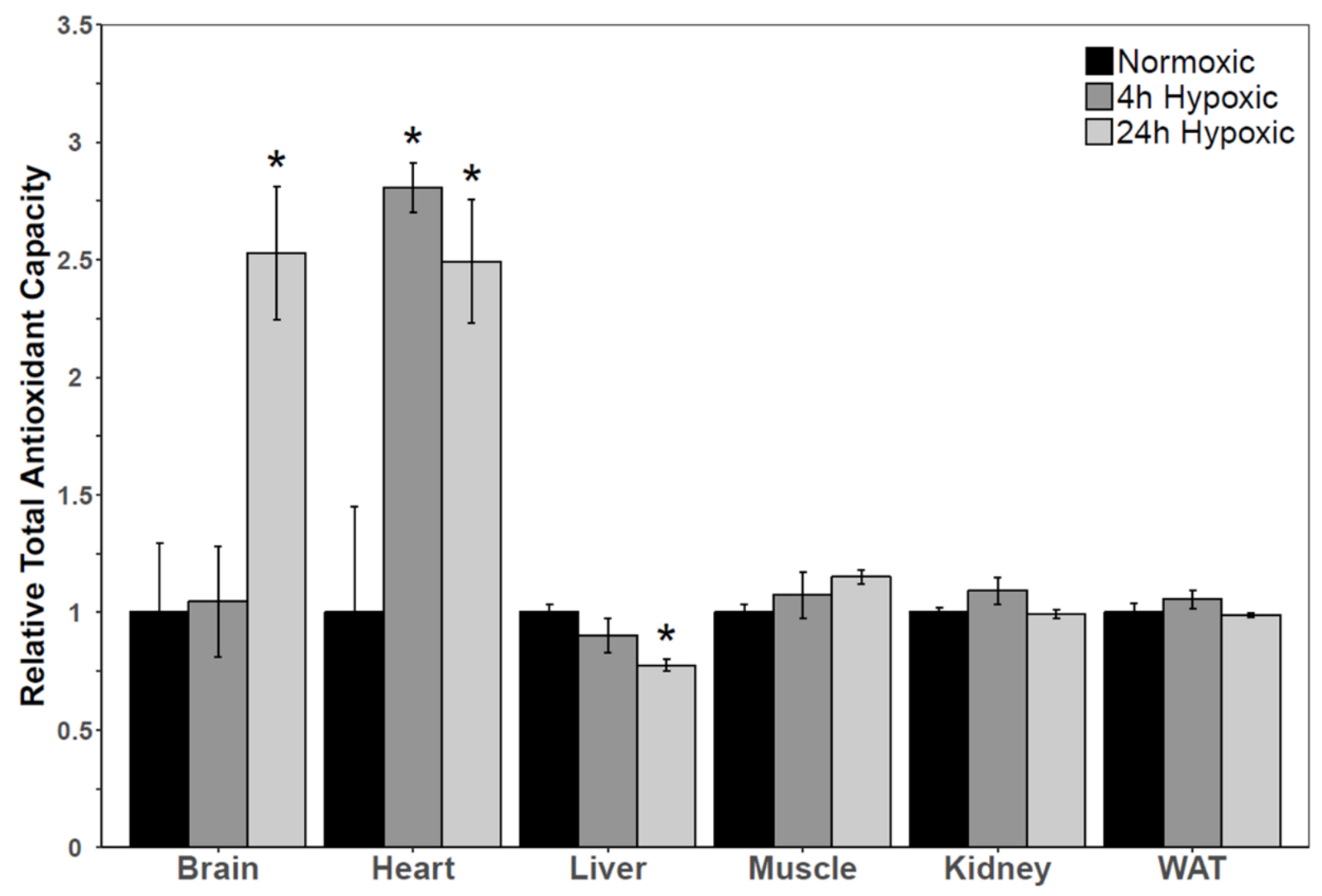

Figure 2.5. Total antioxidant capacity in hypoxic naked mole-rat tissues. Histogram comparing relative antioxidant capacity of normoxic (control), $4 \mathrm{~h}$ hypoxic, and $24 \mathrm{~h}$ hypoxic naked mole-rat tissues (brain, heart, liver, muscle, kidney, WAT). Other information as per Figure 2.1. 
A

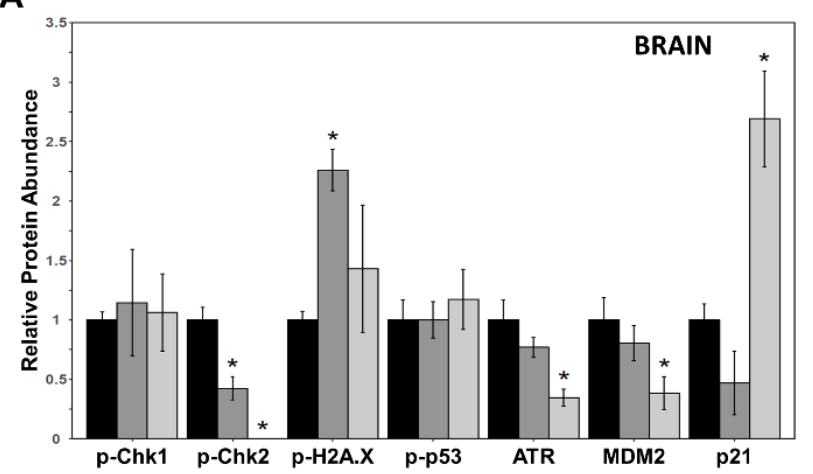

C

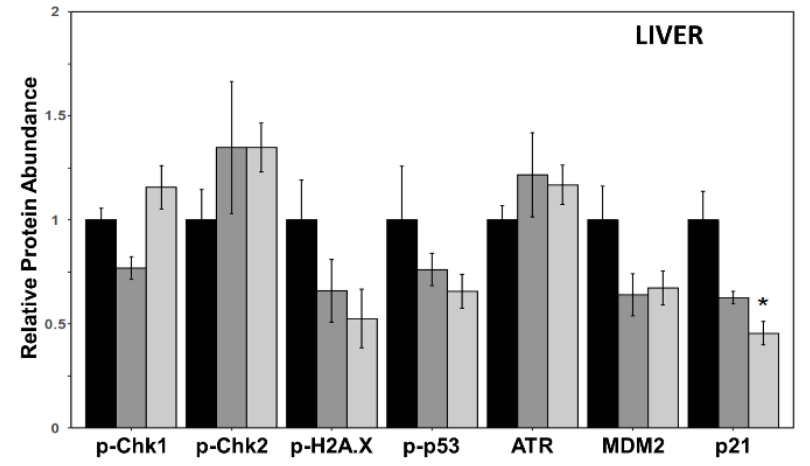

E

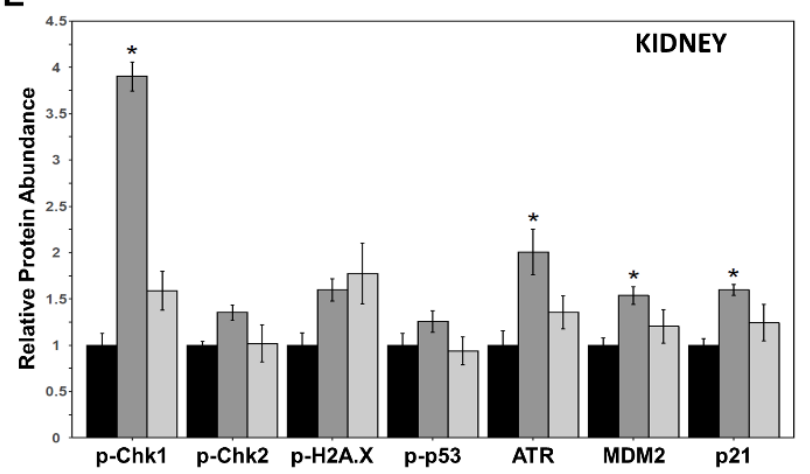

B

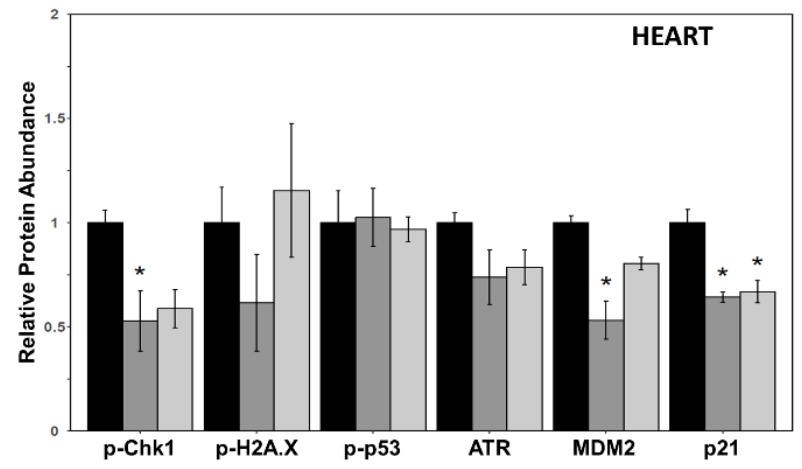

D

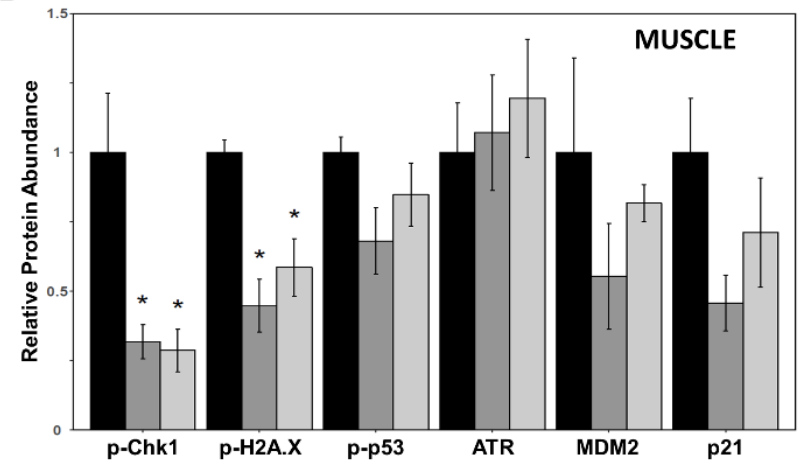

$\mathbf{F}$

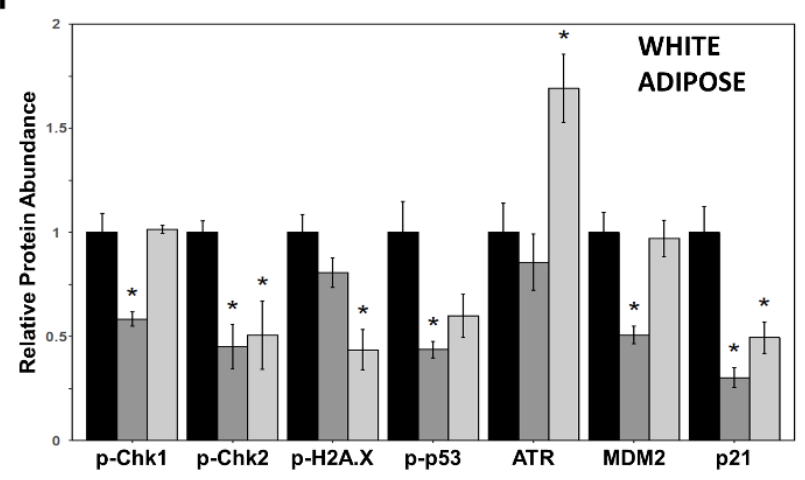

Normoxic $\square$ 4h Hypoxic $\square$ 24h Hypoxic

Figure 2.6. Regulation of DNA damage and repair proteins in hypoxic naked mole-rat tissues. Histograms compare relative protein levels of phosphorylated Chk1 (Ser345), Chk2 (Thr68), H2A.X (Ser139) and p53 (Ser15), as well as total protein levels of ATR, MDM2 and p21. (A) Brain, (B) Heart, (C) Liver, (D) Skeletal muscle, (E) Kidney, and (F) WAT. Other information as per Figure 2.1. 


\section{Chapter 3}

Neuroprotective cell survival proteins 


\section{Naked mole-rats activate neuroprotective proteins during hypoxia}

Liam J. Hawkins ${ }^{\#, 1}$, Hanane Hadj-Moussa ${ }^{\#, 1}$, Vu Chau Nguyen ${ }^{2}$, Mathew E. Pamenter ${ }^{2,3}$, Kenneth B. Storey, 1

${ }^{1}$ Department of Biology, Carleton University, 1125 Colonel By Drive, Ottawa, ON, Canada

${ }^{2}$ Biology Department, University of Ottawa, Marie-Curie Pvt, Ottawa, ON, Canada

${ }^{3}$ Brain and Mind Research Institute, University of Ottawa, Ottawa, ON, Canada

\# Indicates equal contribution

This study has been published in Journal of Experimental Zoology Part A: Ecological and Integrative Physiology:

Hawkins LJ, Hadj-Moussa H, Nguyen VC, Pamenter ME, \& Storey KB. (2019). Naked molerats activate neuroprotective proteins during hypoxia. Journal of Experimental Zoology Part A: Ecological and Integrative Physiology, 331(10), 571-576. 


\subsection{Abstract}

Naked mole-rats are a long-lived animal model that age much like humans, but that can also withstand oxidative damage, cancer, neurodegenerative diseases, and severe hypoxic conditions, which is of particular interest to this study. The conditions of their underground burrows result in competition for oxygen consumption, yet despite this oxygen deprivation they emerge unscathed. To understand the mechanisms in place to facilitate neuronal preservation during hypoxia, we investigated the protein levels of well-known cell-stress factors. We found that under hypoxic conditions, nearly half of the proteins measured increased expression in brain, while only a few decreased. Under hypoxic conditions there appeared to be a HIF1 $\alpha$-centered response, where HIF $1 \alpha$ and its interactors carbonic anhydrase 9, CITED2, p21/CIP1, and NFkB1, among others, were upregulated. Concurrently, a hypoxia-induced decrease of cytochrome $\mathrm{c}$ was consistent with decreased mitochondrial function and protection from apoptosis. The picture that emerges is one of neuroprotection, cell-cycle arrest, and the promotion of anti-apoptotic functions, all of which are consistent with conserving energy and maintaining neural integrity under low oxygen levels. These results suggest how this species may be poised to face hypoxia and contribute to its remarkable ability to deal with myriad other damaging factors and sets the stage for future work on the neuroprotective facilitators we identified.

\subsection{Introduction}

Naked mole-rats are remarkably long-lived mammals and have evolved a suite of adaptations that have led to their cancer resistance, resistance to neurodegeneration, and survival of prolonged oxygen deprivation (Liang et al., 2010; Edrey et al., 2013; Park et al., 2017; Pamenter et al., 2018). Native to East Africa where they live in underground tunnel systems, naked mole-rats are one of 
two eusocial mammalian species and studies have shown to fluctuate body temperature with ambient temperatures (Buffenstein and Yahav, 1991; Ilacqua et al., 2017; Kirby et al., 2018). It is possible that a combination of their heterothermic capacity, along with other molecular adaptations such as their enhanced hemoglobin oxygen binding (Johansen et al., 1976) and their ability to switch to fructose-driven metabolism allow them to endure periods of reduced oxygen availability (Park et al., 2017). The nature of their tunnel colonies make this mammal no stranger to hypoxia such that they can survive for days to weeks at $8 \% \mathrm{O}_{2}$ levels, for hours at 3\%, and up to 18 minutes of complete anoxia (Chung et al., 2016; Park et al., 2017; Pamenter et al., 2018), although how often the animals are exposed to hypoxia in their colonies is controversial (Holtze et al., 2018).

When oxygen is limited, the already low metabolic rates of naked mole-rats are further depressed as they retreat into a hypometabolic state characterized by lowered heart rate, reduced mobility, alternate fuel usage, and lowered body temperature which itself would reinforce altered oxygen affinity and usage (Pamenter et al., 2014, 2019; Chung et al., 2016; Ilacqua et al., 2017; Kirby et al., 2018). While it was originally believed that naked mole-rats avoid oxidative damage as a part of their natural aging process, studies have since shown that naked mole-rats accrue high levels of oxidative damage (Andziak and Buffenstein, 2006; Andziak et al., 2006; Mitchell et al., 2007; Pérez et al., 2009; De Waal et al., 2013). However, this damage is offset by a biochemical organization that is resistant to these harmful effects and that prevents an otherwise unavoidable decline in cognitive function thereby leaving them virtually unscathed (Edrey et al., 2013, 2014). Collectively, these findings imply that extensive networks of neuroplasticity and protective mechanisms could serve a dual use and be in place to protect naked mole-rat brains during hypoxia as well and cell-stress proteins such as HSP70 have been specifically implicated (Edrey et al., 
2014) leading us to ask how the cell-stress related protein landscape changes in response to hypoxia.

Given the evidence of significant neuroprotective mechanisms in this species, the primary objective of this study was to determine which cell-stress related proteins could potentially be involved in protecting naked mole-rat brain function during hypoxia. We therefore exposed naked mole-rats to hypoxia and investigated changes in the protein expression of 26 cell-stress markers between brains of normoxic and hypoxic naked mole-rats. Our results revealed a strong induction of hypoxia-inducible factor $1 \alpha(\mathrm{HIF} 1 \alpha)$, nuclear factor kappa-light-chain-enhancer of activated B cells $(\mathrm{NF \kappa B})$, and multiple pro-survival proteins that appear to promote neuroprotective functions during hypoxic exposures (Figure 3.1). These findings corroborate the established HIF1 $\alpha$-centered mechanism present in hypoxic naked mole-rat brains, as well as implicated novel neuroprotective targets in this system. These results demonstrate yet another layer of biochemical and molecular adaptations that naked mole-rats have putatively developed in response to hypoxia-associated challenges, from which we may gather insight and emulate in the treatment of human conditions related to hypoxia. It should be noted that while this study implicated several pro-survival proteins during hypoxia, since our study focused entirely on relative protein abundance levels, these findings should be further investigated to examine whether these candidate hypoxic neuroprotectors actually afford protection in these animals.

\subsection{Materials and Methods}

\subsection{1 $\quad$ Animals}

Adult male naked mole-rats (Heterocephalus glaber) weighing approximately $40 \mathrm{~g}$ were obtained from a group-housed colony at the University of Ottawa. Animal feeding, monitoring, 
behaviour, and conditions of hypoxic exposures were as described previously (Houlahan et al., 2018).

\subsubsection{Hypoxia protocol}

Eight subordinate and non-breeding males were placed into an experimental chamber. Animals were provided with a thin layer of bedding on the floor of the experimental chambers. The animal chamber was sealed and constantly ventilated with gas mixtures, set to the desired fractional gas composition by calibrated rotameters (Praxair, Mississauga, ON, CA). Inflowing gas was provided at a flow rate of $0.5 \mathrm{~L} / \mathrm{min}$, as assessed by a calibrated mass flow meter (Alicat Scientific, Tucson, AZ, USA). Four normoxic (control; 20.95\% $\mathrm{O}_{2}, 0.05 \% \mathrm{CO}_{2}$, balance $\mathrm{N}_{2}$ ) animals were euthanized by conscious cervical dislocation followed immediately by decapitation. The incurrent gas was switched to $7 \% \mathrm{O}_{2}\left(0.05 \% \mathrm{CO}_{2}\right.$, balance $\left.\mathrm{N}_{2}\right)$ and the flow rate was increased to $1 \mathrm{~L} / \mathrm{min}$ for $10 \mathrm{~min}$ to rapidly reduce the $\mathrm{O}_{2}$ content of the chamber to $7 \% \mathrm{O}_{2}$, and were then reset to $0.5 \mathrm{~L} / \mathrm{min}$ for the remainder of the exposure. Oxygen levels were continuously monitored throughout the experiment using a FC-10 $\mathrm{O}_{2}$ analyzer (Sable Systems International, Las Vegas, $\mathrm{NV}$, USA). Four hypoxic animals remained in the chamber for $4 \mathrm{~h}$, followed by cervical dislocation and decapitation. Animals were rapidly dissected, whole brains, excluding the brainstem, were excised within $30 \mathrm{sec}$, and were then flash frozen in liquid nitrogen, and stored at $-80{ }^{\circ} \mathrm{C}$ until analysis. All naked mole-rat experiments were in compliance with the requirements proposed by the Canadian Council on Animal Care and were approved by the University of Ottawa Animal Care Committee. Animal care protocol [\#2535].

\subsubsection{Protein isolations}

Protein extractions were performed as per the manufacturer's instructions (R\&D Systems; ARY018). Samples of frozen brain $(\sim 100 \mathrm{mg})$ were homogenized in phosphate-buffered saline 
with protease inhibitors and then $7 \%$ Triton X-100 was added. Homogenized samples were then frozen at $-80^{\circ} \mathrm{C}$, thawed, and centrifuged at $10,000 \times \mathrm{g}$ for $5 \mathrm{~min}$. Supernatants were collected and total protein concentrations were measured using the Bradford assay (Bio-Rad; 500-0006) (Bradford, 1976). Protein lysates were assayed immediately to avoid freeze-thaw cycles.

\subsubsection{Antibody array profiling}

The membrane-based R\&D Systems Sandwich Immunoassay Human Cell Stress Proteome Profiler Array Kit was used to profile the relative abundance of 26 cell-stress related proteins. The assay was performed according to the manufacturers directions (R\&D Systems; ARY018). The protein targets analyzed were: a disintegrin and metalloproteinase with thrombospondin motifs 1 (ADAMTS1), B-cell CLL/lymphoma 2 (BCL2), carbonic anhydrase 9 (CA9), CBP/P300interacting transactivator 2 (CITED2), cytochrome c oxidase 2 (COX2), Cytochrome c (CYC), Dickkopf WNT signaling pathway inhibitor 4 (DKK4), fatty-acid binding protein 1 (FABP1), hypoxia-inducible factor $1 \alpha$ (HIF1 $\alpha$ ), hypoxia-inducible factor $2 \alpha$ (HIF2 $\alpha$ ), phosphorylated heat shock protein 27 (phospho-HSP27 [S78/S82]), heat shock protein 60 (HSP60), heat shock protein 70 (HSP70), indoleamine 2,3-dioxygenase (IDO), phosphorylated JUN N-terminal kinase (phospho-JNK [T183/Y185]), nuclear factor kappa B subunit 1 (NFKB1), CDK-interacting protein 1 (p21/CIP1), p27/Kip1, phospho-p38a [T180/Y182], phospho-p53 [S46], paraoxonase 1 (PON1),

paraoxonase 2 (PON2), paraoxonase 3 (PON3), sirtuin 2 (SIRT2), superoxide dismutase 2 (SOD2), and thioredoxin-1 (TXN).

\subsubsection{Data analysis}

Membranes were visualized with the provided enhanced chemiluminescence solution using a Chemi-Genius Syngene Bio-Imaging System (Syngene, Frederick, MD, USA). Protein spot densities were quantified using Gene Tools software. Protein target densities for each spot were 
subtracted from an averaged background signal and normalized to controls on each membrane. Data are expressed as mean change $\pm \operatorname{SEM}(n=4$ each), relative to controls. Data were analyzed using a Student's $t$-test and FDR corrected $\left({ }^{*} p<0.05\right)$ using the p.adjust R-package (https://stat.ethz.ch/R-manual/R-devel/library/stats/html/p.adjust.html) which implements the Benjamini-Hochberg procedure (Benjamini and Hochberg, 1995) to compare proteins levels from normoxic and hypoxic animals. Statistical analysis and figure generation was performed using the SigmaPlot 12.5 statistics and graphing software. Figure 3.1 was generated using illustrations from BioRender (https://biorender.com).

\subsection{Results and Discussion}

To be able to accumulate profound oxidative damage and yet remain unscathed has rendered naked mole-rats an excellent mammalian model of ageing and we believe these characteristics also make them excellent candidate models for mammalian hypoxia tolerance. When humans are faced with oxygen deprivation, dealing with the damage that ensues in a naked mole-rat like manner is something we can strive for in the development of therapeutics. The 26 cell-stress related proteins we examined span a broad range of protective biological functions including cell cycle regulation, apoptotic controls, proteome stabilization, and antioxidant defences. Of these, 14 proteins were differentially regulated in response to hypoxia (Figure 3.2A). The 11 hypoxia-upregulated proteins (PON3, HIF1a, DKK4, CITED2, SIRT2, CA9, p21, IDO, NFKB1, p-HSP27, and FABP1) appeared to be promoting an anti-apoptotic and cytoprotective response to attend to the hypoxiainduced oxidative stress that is likely present (Figure 3.2B; Supp. Table S1). This functional trend was also evident in the three hypoxia-downregulated proteins (HSP70, p-JNK, and CYC), where the reduced expression of p-JNK and CYC in particular have been linked to enhanced 
neuroprotection (Figure 3.2B; Supp. Table S1) (Cai et al., 1998; Okami et al., 2013). It is interesting to note that while all of the 26 proteins examined in this study play known roles in cell stress, the fact that not all were differentially regulated in hypoxia is likely due to the metabolic rate depression naked mole-rats are experiencing. It is possible that this hypometabolic state forces them to preferentially rely on a subset of proteins and mechanisms to limit unnecessary energetic expenditures as many of these proteins display redundant protective functions.

From the proteins examined herein, the $3.39 \pm 0.31$-fold increase of the transcription factor HIF $1 \alpha$ in hypoxic naked mole-rat brains stands out (Figure 3.2B). It is well-established that while constitutively expressed and degraded under normoxic conditions, during hypoxia HIF1 $\alpha$ stability and activity increase to induce the transcription of over 100 downstream genes involved in angiogenesis, cell cycle regulation, and glucose metabolism, thus providing a mechanism for cells to rapidly adjust to low oxygen availability (Masoud and Li, 2015; Slemc and Kunej, 2016; Cimmino et al., 2019). Previous work has characterized the role of HIF1 $\alpha$ in hypoxic naked molerats and the fact that our broad cell survival screen also strongly implicated HIF1 $\alpha$ further corroborates its importance in this system. naked mole-rats have evolved a HIF1 $\alpha$ protein with a unique mutation that relieves the constant state of HIF $\alpha$ protein degradation and that is specifically adapted to chronic low oxygen availability (Kim et al., 2011). While the upregulation of HIF1 $\alpha$ observed herein did not match the downregulation of HIF1 $\alpha$ in a similar timeframe for $5 \% \mathrm{O}_{2}$ exposed naked mole-rats in a previous study, it did match HIF $1 \alpha$ levels when the animals were exposed to $5 \% \mathrm{O}_{2}$ for $9 \mathrm{~h}$ and longer (Xiao et al., 2017). Thus, increased HIF1 $\alpha$ may be a robust naked mole-rat response to hypoxia with temporal and oxygen specific nuances. Both of these results signal that exposure for less than $4 \mathrm{~h}$ to hypoxic conditions may show interesting HIF $1 \alpha$ dynamics, after which HIF1 $\alpha$ shows consistent upregulation. An interesting naked mole- 
rat specific adaptation reported by Xiao and colleagues was that HIF1 $\alpha$ was expressed at a higher level in naked mole-rat brains as compared to mouse brains, possibly contributing to their elevated hypoxia tolerance and likely due to their unique HIF $1 \alpha$ protein sequence (Xiao et al., 2017). This activation of HIF $1 \alpha$ has also been observed in other animal models of metabolic rate depression and anoxia/hypoxia tolerance, an observation that has been made at the protein, mRNA, and noncoding RNA levels (Gorr, 2004; Morin et al., 2005; Maistrovski et al., 2012; English et al., 2018).

Many of the proteins evaluated in this study have previously been shown to be under the control of HIF $1 \alpha$ and to exhibit similar increases to that of HIF $1 \alpha$ under hypoxia, suggesting a HIF $1 \alpha$-centric response (Figure 3.2B) (Masoud and Li, 2015; Cimmino et al., 2019). For example, there is a HIF $1 \alpha$ response element in the promoter of CA9 (Wykoff et al., 2000); the enzyme that balances cellular $\mathrm{pH}$ in response to the acidifying effect of hypoxia (Pamenter et al., 2019). As seen in our results, CA9 increases $>2.5$-fold in response to hypoxia, which appears consistent with the hypoxia tolerant phenotype of naked mole-rats (Figure 3.2B). Another downstream target that was likely subject to HIF1 $\alpha$ transcriptional activation under hypoxia was CITED2. This transcription factor is responsible for the reduction of pro-apoptotic genes downstream of HIF $1 \alpha$ and the activation of pro-survival genes, thereby promoting a cytoprotective role for HIF $1 \alpha$ (Bakker et al., 2007). P21/CIP1, a protein that was increased 2.52-fold \pm 0.15 under hypoxia (Figure 3.2B), is also a HIF1 $\alpha$ target gene and has previously been shown to be transcribed in response to hypoxia in neurons (Mergenthaler et al., 2013). This cyclin-dependant kinase inhibitor has also been shown to contribute to cell cycle arrest in response to stress in a p53-dependant manner (Lewis et al., 2012). Inhibiting the progression of the neuronal cell cycles partially through the upregulation of $\mathrm{p} 21 / \mathrm{CIP} 1$ under hypoxia could help naked mole-rats limit ATP-expensive processes while metabolic and aerobic capabilities are suppressed. 
Also activated in hypoxic naked mole-rat brains was the redox-sensitive transcription factor $\mathrm{NF \kappa B}$, which increased $2.43 \pm 0.18$-fold (Figure 3.2B). It would be beneficial for $\mathrm{NF \kappa B}$ to be upregulated in hypoxic naked mole-rat brains as this transcription factor is considered one of the first molecular responders to oxidative stress, where its activation results in decreased apoptosis and increased angiogenesis (D'Ignazio and Rocha, 2016). This NFKB activation is the center of antioxidant defenses, inflammatory responses, and DNA damage repair and is likely contributing to the cytoprotection that could be happening in naked mole-rat brains to avoid or minimize injury when oxygen availability is limited (Hayden and Ghosh, 2012). Although the exact mechanism for NFKB induction under hypoxia is still not clear (D'Ignazio and Rocha, 2016), it has been shown to cross-talk with both HIF $1 \alpha$ and with many HIF $1 \alpha$ regulating proteins, providing additional support to the observed HFI1 $\alpha$-centric response (D'Ignazio and Rocha, 2016).

While most of the examined proteins either increased or showed no statistical difference, three were found to significantly decrease in hypoxic brains (Figure 3.2A), with cytochrome C (CYC) displaying the greatest reduction in protein level (Figure 3.2B). CYC is a necessary component of the mitochondrial electron transport chain, and its reduction is consistent with the reduced flux through the ETC in hypoxic naked mole-rat brains (Pamenter et al., 2018). Moreover, CYC also serves a role in signalling apoptosis when released from the mitochondria into the cytosol (Cai et al., 1998); therefore, besides matching lowered oxidative phosphorylation capacity, the reduction of CYC may also reduce the apoptotic potential of naked mole-rat brain cells during hypoxia.

\subsection{Conclusion}

The overall trend that emerges from this study is a picture of robust neuroprotection and an anti-apoptotic response to hypoxia in naked mole-rat brain. The functions of many of the observed differentially expressed proteins span multiple protective pathways and suggest that the 
neuroprotective phenotype is intimately related to the global hypoxia tolerance. Perhaps unsurprisingly, our examination of a broad screen of known pro-survival proteins implicated, once again, HIF $1 \alpha$ regulation in hypoxic naked mole-rats, but for the first time, we are able to examine this previously reported result in the broader context of other survival networks. While this study provides us with a snapshot of the neuroprotective regulatory mechanisms in place to facilitate hypoxic brain exposures, the relatively small number of animals used and the lack of functional assays performed limits the functional global conclusions that can be drawn and rather sets the stage for future studies to follow up on the neuroprotective targets identified. Indeed, understanding how this species is poised to mount such a rapid response to hypoxia may elucidate mechanisms useful for application to various biomedical problems. 


\subsection{References}

Andziak B, Buffenstein R. 2006. Disparate patterns of age-related changes in lipid peroxidation in long-lived naked mole-rats and shorter-lived mice. Aging Cell. 5:525-32.

Andziak B, O'Connor TP, Qi W, DeWaal EM, Pierce A, Chaudhuri AR, Van Remmen H, Buffenstein R. 2006. High oxidative damage levels in the longest-living rodent, the naked mole-rat. Aging Cell. 5:463-71.

Bakker WJ, Harris IS, Mak TW. 2007. FOXO3a is activated in response to hypoxic stress and inhibits HIF1-induced apoptosis via regulation of CITED2. Mol Cell. 28:941-53.

Benjamini Y, Hochberg Y. 1995. Controlling the False Discovery Rate : A Practical and Powerful Approach to Multiple Testing. J R Stat Soc Ser B. 57:289-300.

Bradford MM. 1976. A rapid and sensitive method for the quantitation of microgram quantities of protein utilizing the principle of protein-dye binding. Anal Biochem. 72:248-54.

Buffenstein R, Yahav S. 1991. Is the naked mole-rat Hererocephalus glaber an endothermic yet poikilothermic mammal? J Therm Biol. 16:227-232.

Cai J, Yang J, Jones D. 1998. Mitochondrial control of apoptosis: the role of cytochrome c. Biochim Biophys Acta - Bioenerg. 1366:139-149.

Chung D, Dzal YA, Seow A, Milsom WK, Pamenter ME. 2016. Naked mole-rats exhibit metabolic but not ventilatory plasticity following chronic sustained hypoxia. Proceedings Biol Sci. 283:20160216.

Cimmino F, Avitabile M, Lasorsa VA, Montella A, Pezone L, Cantalupo S, Visconte F, Corrias MV, Iolascon A, Capasso M. 2019. HIF-1 transcription activity: HIF1A driven response in normoxia and in hypoxia. BMC Med Genet. 20:1-15.

D’Ignazio L, Rocha S. 2016. Hypoxia induced NF-кB. Cells. 5:10.

Edrey YH, Medina DX, Gaczynska M, Osmulski PA, Oddo S, Caccamo A, Buffenstein R. 2013. Amyloid beta and the longest-lived rodent: the naked mole-rat as a model for natural protection from Alzheimer's disease. Neurobiol Aging. 34:2352-60.

Edrey YH, Oddo S, Cornelius C, Caccamo A, Calabrese V, Buffenstein R. 2014. Oxidative damage and amyloid- $\beta$ metabolism in brain regions of the longest-lived rodents. J Neurosci Res. 92:195-205.

English SG, Hadj-Moussa H, Storey KB. 2018. MicroRNAs regulate survival in oxygen-deprived environments. J Exp Biol. 221:jeb.190579.

Gorr TA. 2004. Daphnia and Drosophila: two invertebrate models for O2 responsive and HIFmediated regulation of genes and genomes. Int Congr Ser. 1275:55-62. 
Hayden MS, Ghosh S. 2012. NF- $\kappa B$, the first quarter-century: remarkable progress and outstanding questions. Genes Dev. 26:203-34.

Houlahan CR, Kirby AM, Dzal YA, Fairman GD, Pamenter ME. 2018. Divergent behavioural responses to acute hypoxia between individuals and groups of naked mole-rats. Comp Biochem Physiol B Biochem Mol Biol. 224:38-44.

Ilacqua AN, Kirby AM, Pamenter ME. 2017. Behavioural responses of naked mole-rats to acute hypoxia and anoxia. Biol Lett. 13:1-4.

Johansen K, Lykkeboe G, Weber R, Maloiy G. 1976. Blood respiratory properties in the naked mole-rat Heterocephalus glaber, a mammal of low body temperature. Respir Physiol 28:303314.

Kim EB, Fang X, Fushan AA, Huang Z, Lobanov A V., Han L, Marino SM, Sun X, Turanov AA, Yang P, Yim SH, Zhao X, Kasaikina M V., Stoletzki N, Peng C, Polak P, Xiong Z, Kiezun A, Zhu Y, Chen Y, Kryukov G V., Zhang Q, Peshkin L, Yang L, Bronson RT, Buffenstein R, Wang B, Han C, Li Q, Chen L, Zhao W, Sunyaev SR, Park TJ, Zhang G, Wang J, Gladyshev VN. 2011. Genome sequencing reveals insights into physiology and longevity of the naked mole-rat. Nature. 479:223-227.

Kirby AM, Fairman GD, Pamenter ME. 2018. Atypical behavioural, metabolic and thermoregulatory responses to hypoxia in the naked mole-rat (Heterocephalus glaber). J Zool. 305:106-115.

Lewis KN, Mele J, Hornsby PJ, Buffenstein R. 2012. Stress resistance in the naked mole-rat: the bare essentials - a mini-review. Gerontology. 58:453-62.

Liang S, Mele J, Wu Y, Buffenstein R, Hornsby PJ. 2010. Resistance to experimental tumorigenesis in cells of a long-lived mammal, the naked mole-rat (Heterocephalus glaber). Aging Cell. 9:626-35.

Maistrovski Y, Biggar KK, Storey KB. 2012. HIF-1 $\alpha$ regulation in mammalian hibernators: role of non-coding RNA in HIF-1 $\alpha$ control during torpor in ground squirrels and bats. J Comp Physiol B. 182:849-59.

Masoud GN, Li W. 2015. HIF-1 $\alpha$ pathway: role, regulation and intervention for cancer therapy. Acta Pharm Sin B. 5:378-89.

Mergenthaler P, Muselmann C, Sünwoldt J, Isaev NK, Wieloch T, Dirnagl U, Meisel A, Ruscher K. 2013. A functional role of the cyclin-dependent kinase inhibitor 1 (p21(WAF1/CIP1)) for neuronal preconditioning. J Cereb Blood Flow Metab. 33:351-5.

Mitchell TW, Buffenstein R, Hulbert AJ. 2007. Membrane phospholipid composition may contribute to exceptional longevity of the naked mole-rat (Heterocephalus glaber): a comparative study using shotgun lipidomics. Exp Gerontol. 42:1053-62.

Morin P, McMullen DC, Storey KB. 2005. HIF-1 $\alpha$ involvement in low temperature and anoxia 
survival by a freeze tolerant insect. Mol Cell Biochem. 280:99-106.

Okami N, Narasimhan P, Yoshioka H, Sakata H, Kim GS, Jung JE, Maier CM, Chan PH. 2013. Prevention of JNK phosphorylation as a mechanism for rosiglitazone in neuroprotection after transient cerebral ischemia: activation of dual specificity phosphatase. J Cereb Blood Flow Metab. 33:106-14.

Pamenter ME, Dzal YA, Milsom WK. 2014. Adenosine receptors mediate the hypoxic ventilatory response but not the hypoxic metabolic response in the naked mole-rat during acute hypoxia. Proc R Soc B Biol Sci. 282:20141722-20141722.

Pamenter ME, Dzal YA, Thompson WA, Milsom WK. 2019. Do naked mole-rats accumulate a metabolic acidosis or an oxygen debt in severe hypoxia? J Exp Biol. 222.

Pamenter ME, Lau GY, Richards JG, Milsom WK. 2018. Naked mole-rat brain mitochondria electron transport system flux and $\mathrm{H}+$ leak are reduced during acute hypoxia. J Exp Biol. 221:jeb.171397.

Park TJ, Reznick J, Peterson BL, Blass G, Omerbašić D, Bennett NC, Kuich PHJL, Zasada C, Browe BM, Hamann W, Applegate DT, Radke MH, Kosten T, Lutermann H, Gavaghan V, Eigenbrod O, Bégay V, Amoroso VG, Govind V, Minshall RD, Smith ESJ, Larson J, Gotthardt M, Kempa S, Lewin GR. 2017. Fructose-driven glycolysis supports anoxia resistance in the naked mole-rat. Science. 356:307-311.

Pérez VI, Buffenstein R, Masamsetti V, Leonard S, Salmon AB, Mele J, Andziak B, Yang T, Edrey Y, Friguet B, Ward W, Richardson A, Chaudhuri A. 2009. Protein stability and resistance to oxidative stress are determinants of longevity in the longest-living rodent, the naked mole-rat. Proc Natl Acad. Sci U S A 106:3059-64.

De Waal EM, Liang H, Pierce A, Hamilton RT, Buffenstein R, Chaudhuri AR. 2013. Elevated protein carbonylation and oxidative stress do not affect protein structure and function in the long-living naked-mole-rat: A proteomic approach. Biochem Biophys Res Commun. 434:815-819.

Wykoff CC, Beasley NJ, Watson PH, Turner KJ, Pastorek J, Sibtain A, Wilson GD, Turley H, Talks KL, Maxwell PH, Pugh CW, Ratcliffe PJ, Harris AL. 2000. Hypoxia-inducible expression of tumor-associated carbonic anhydrases. Cancer Res. 60:7075-83.

Xiao B, Wang S, Yang G, Sun X, Zhao S, Lin L, Cheng J, Yang W, Cong W, Sun W, Kan G, Cui S. 2017. HIF-1 $\alpha$ contributes to hypoxia adaptation of the naked mole-rat. Oncotarget. 8:109941-109951. 


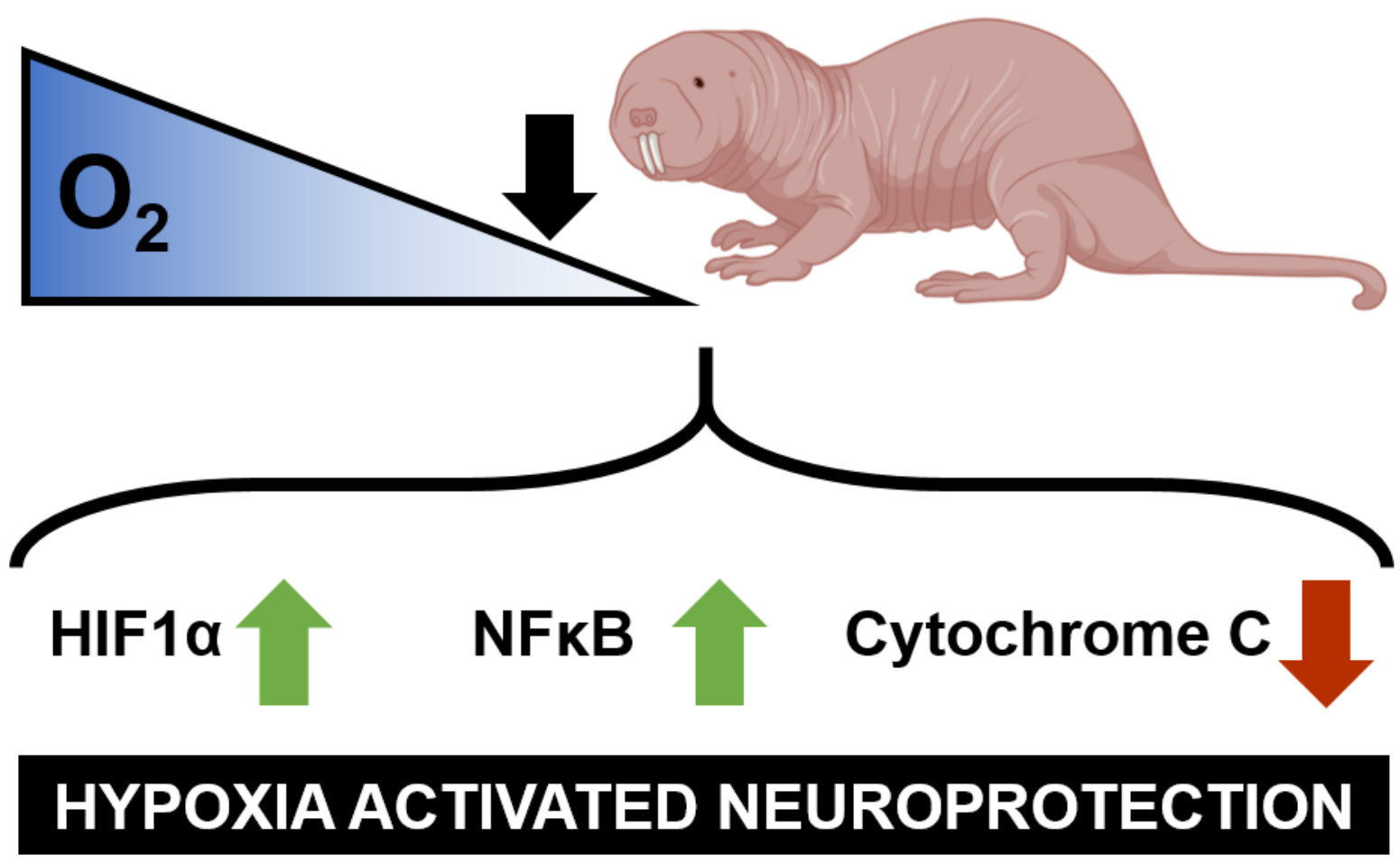

Figure 3.1. Summary of the central pro-survival protein changes observed in hypoxic naked mole-rat brains. 
A

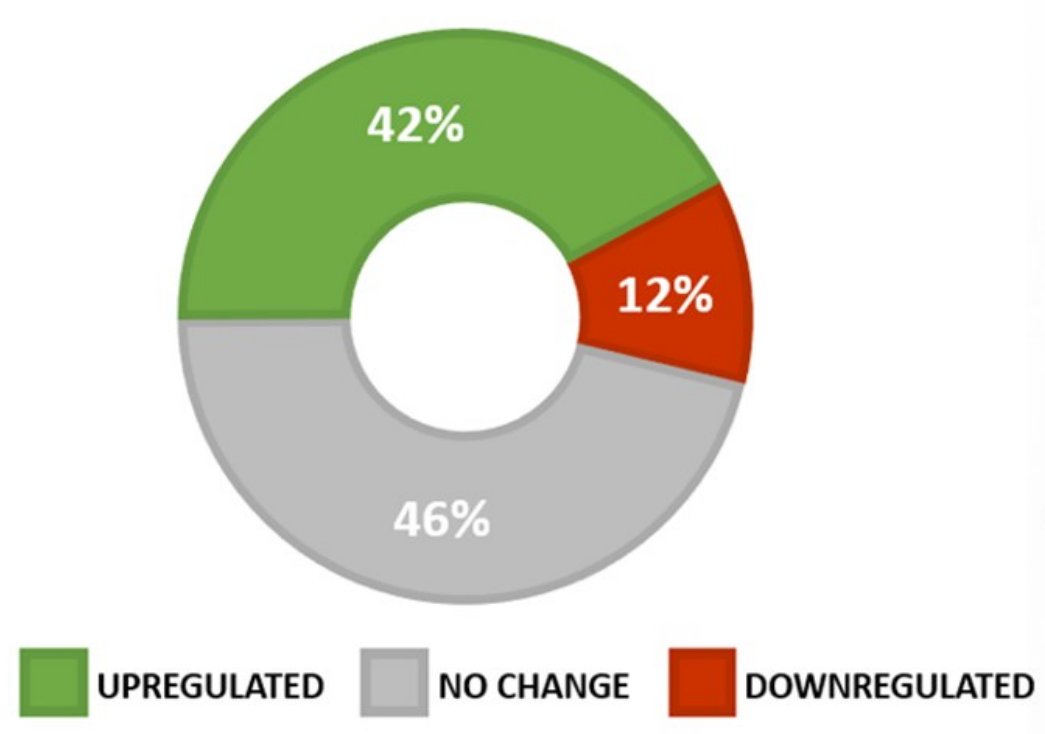

B

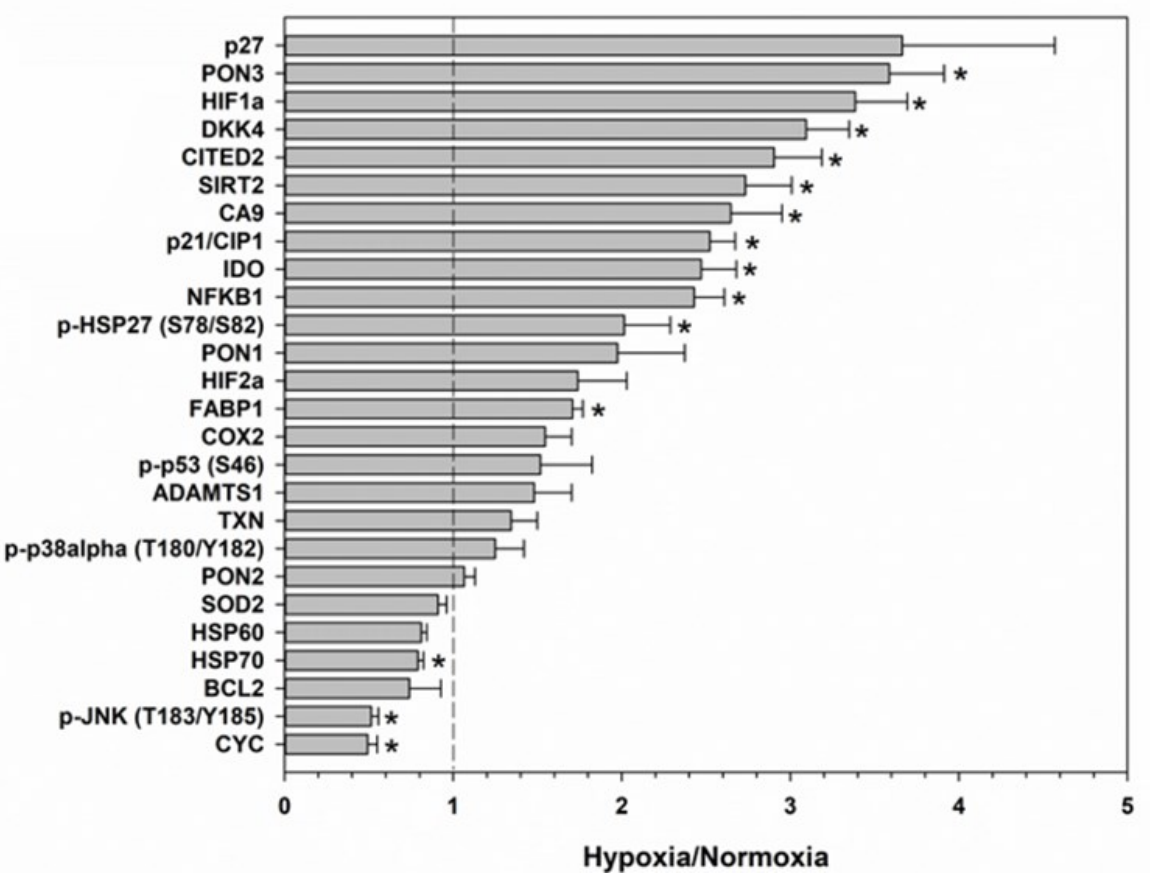

Figure 3.2. Cell-stress related protein levels in response to hypoxia in the brain of naked mole-rats. [A] Overview of measured protein level changes. Of the proteins measured, 42\% (11/26) were upregulated in the hypoxic condition, 46\% (12/26) did not change statistically, and 12\% (3/26) proteins were downregulated. [B] Hypoxia to normoxia protein level ratios for 26 cell-stress related proteins. Data are mean $\pm \mathrm{SEM}, *$ indicates statistically significant differences between normoxic and hypoxic animals $(p$ $<0.05)$ as determined by Student's $t$-test with FDR correction. Eight animals were used in total, four in the normoxic group and 4 in the hypoxic group. 


\subsection{Supplementary Materials}

The data supporting this article is present in the figures and has been uploaded in full to Supplementary Table S1 which can be found here:

Hawkins LJ*, Hadj-Moussa H*, Nguyen VC, Pamenter ME, \& Storey KB. (2019). Naked mole-rats activate neuroprotective proteins during hypoxia. Journal of Experimental Zoology Part A: Ecological and Integrative Physiology, 331(10), 571-576.

Supplementary Table S1. Relative abundance of cell survival proteins in normoxic (control) and hypoxic naked mole-rat brains. 
Chapter 4

\section{Hypoxic miRNAs switch fuels and protect brains}




\section{Hypoxic naked mole-rat brains use microRNA to coordinate hypometabolic fuels and neuroprotective defenses}

Hanane Hadj-Moussa ${ }^{1}$, Matthew E. Pamenter ${ }^{2,3}$, and Kenneth B. Storey ${ }^{1}$

${ }^{1}$ Department of Biology, Carleton University, 1125 Colonel By Drive, Ottawa, ON, Canada

${ }^{2}$ Biology Department, University of Ottawa, Marie-Curie Pvt, Ottawa, ON, Canada

${ }^{3}$ Brain and Mind Research Institute, University of Ottawa, Ottawa, ON, Canada

This study is published in the Journal of Cellular Physiology:

Hadj-Moussa H, Pamenter ME, \& Storey KB. (2021). Hypoxic naked mole-rat brains use microRNA to coordinate hypometabolic fuels and neuroprotective defenses. Journal of Cellular Physiology, 236(7), 5080-5097. 


\subsection{Abstract}

Naked mole-rats are among the mammalian champions of hypoxia tolerance. They evolved adaptations centered around reducing metabolic rate to overcome the challenges experienced in their underground burrows. In this study, we used next-generation sequencing to investigate one of the factors likely supporting hypoxia tolerance in naked mole-rat brains, post-transcriptional microRNAs. Of the 212 conserved microRNAs identified using small RNA sequencing, 18 displayed significant differential expression during hypoxia. Bioinformatic enrichment revealed that hypoxia-mediated microRNAs were suppressing energy expensive processes including de novo protein translation and cellular proliferation. This suppression occurred alongside the activation of neuroprotective and neuroinflammatory pathways, and the induction of central signal transduction pathways including HIF-1 $\alpha$ and NFkB via miR-335, miR-101, and miR-155. MicroRNAs also coordinated anaerobic glycolytic fuel sources, where hypoxia-upregulated miR365 likely suppressed protein levels of ketohexokinase, the enzyme responsible for catalyzing the first committed step of fructose catabolism. This was further supported by a hypoxia-mediated reduction in glucose transporter 5 proteins that import fructose into the cell. Yet, mRNA and protein levels of lactate dehydrogenase, which converts pyruvate to lactate in the absence of oxygen, were elevated during hypoxia. Together, this demonstrated the induction of anaerobic glycolysis despite a lack of reliance on fructose as the primary fuel source, suggesting that hypoxic brains are metabolically different than anoxic naked mole-rat brains that were previously found to shift to fructose-based glycolysis. Our findings contribute to the growing body of oxygenresponsive miRNAs 'OxymiRs' that facilitate natural microRNA-mediated mechanisms for successful hypoxic exposures. 


\subsection{Introduction}

While humans are surprisingly adept at coping with low oxygen levels for a short time, prolonged oxygen deprivation leads to numerous maladies and can lead to death. This is in stark contrast to the naked mole-rat's (Heterocephalus glaber) ability to overcome the challenges of prolonged hypoxic exposures. Not only are naked mole-rats one of the most hypoxia tolerant mammals, they are emerging as an important model for research in longevity, cancer resistance, and pain management (Edrey, Hanes, Pinto, Mele, \& Buffenstein, 2011; Smith, Schuhmacher, \& Husson, 2015), all of which add to the impressive arsenal of unique and unusual adaptation they have evolved. Our study focuses on the responses mounted by naked mole-rats to overcome oxygen limitations in the brain.

Native to underground colonies in East Africa, these eusocial mammals live in crowded tunnel systems that experience hypoxia and hypercapnia within their nest chambers (Holtze et al., 2018). While the degree of hypoxia that naked mole-rats are naturally exposed to is a disputed topic, lab studies have shown that they are capable of surviving prolonged hypoxic exposures for days to weeks at $8 \% \mathrm{O}_{2}$ levels and for hours at $3 \% \mathrm{O}_{2}$ (Chung, Dzal, Seow, Milsom, \& Pamenter, 2016; Pamenter, Lau, Richards, \& Milsom, 2018). In vitro studies have shown that isolated naked molerat brain tissues are both hypoxia and anoxia tolerant (Larson \& Park, 2009). Even more phenomenally, naked mole-rats have recently been reported to survive 18 minutes of complete anoxia, where despite losing consciousness after $\sim 30$ seconds of complete anoxia they fully recover after the re-introduction of normoxic conditions. In contrast, mice were killed $\sim 45$ seconds into the anoxic exposure (Larson \& Park, 2009; Park et al., 2017). Unlike mice, naked mole-rats are equipped with various mechanisms that facilitate hypoxic survival, yet key questions regarding these adaptations remain unanswered. 
Many hypoxia and anoxia tolerant species overcome prolonged oxygen-limitation by dropping their metabolic demand to match reduced oxygen availability, allowing energy demands to be met by less efficient anaerobic pathways. This is linked with the simultaneous induction of various antioxidant and pro-survival mechanisms to mitigate ensuing oxidative damage (Hochachka, Buck, Doll, \& Land, 1996; Michael \& Philip, 1999). A focus on naked mole-rat molecular adaptations reveals a reliance on profound metabolic rate depression, along with reductions in physiological and behavioural variables including breathing frequency, heart rate, behavioural activity, and body temperature to tolerate reduced oxygen levels (Pamenter, Dzal, Thompson, \& Milsom, 2019). In the face of this robust downregulation of ATP-consumption, naked mole-rats shift their reliance to carbohydrates when oxygen is limited. Naked mole-rat brains in particular have been shown to switch to anaerobic glycolysis with an emphasis on fructose metabolism as a mechanism to circumvent glycolytic feedback inhibition whilst also accumulating lactate as the final end-product (Park et al., 2017). Pro-survival adaptations include the activation of Hypoxia Inducible Factor 1 Subunit Alpha (HIF-1 $\alpha$ ) and nuclear factor kappalight-chain-enhancer of activated $\mathrm{B}$ cells $(\mathrm{NF \kappa B})$ among other central transcription factors (Hawkins, Hadj-Moussa, Nguyen, Pamenter, \& Storey, 2019; Xiao et al., 2017). But what remains largely unknown are the molecular mechanisms coordinating these sweeping metabolic changes. Our research on other animal models of hypoxia and anoxia tolerance tells us that microRNAs play a role in orchestrating hypoxic protection and metabolic regulation. This study delves into the possible roles of non-coding RNAs in modulating naked mole-rat hypoxic survival.

Non-coding RNA networks have been implicated in both adaptive and maladaptive responses to hypoxia and anoxia exposures. Specifically, microRNA (miRNA), small non-coding RNAs that post-transcriptionally regulate gene expression by either temporarily suppressing mRNA 
translation via transcript storage, or through the permanent degradation of mRNA transcripts (Bartel, 2004). MiRNAs are a class of regulatory molecules that have been shown to target all aspects of biological functions, rendering them excellent candidates for controlling hypometabolism and hypoxia tolerance. Depending on the degree of miRNA complementary binding with the target mRNA, the RNA-induced silencing complex will either direct the transcript to translational repression and storage in cytoplasmic stress granules and processing bodies when binding is imperfect, or degradation when complementation is perfect (O'Brien, Hayder, Zayed, \& Peng, 2018). Five key inherent miRNA properties allow them to orchestrate metabolic reprogramming: [1] they can target all biological processes since a single miRNA can target multiple mRNA transcripts and single mRNA transcripts can be under the regulation of more than one miRNA species, [2] their regulation is reversible, [3] their effects are rapid, [4] they are energetically inexpensive to synthesize, especially when compared to protein regulators, and [5] miRNA sequences are highly conserved among even the most disparate of species (Hadj-Moussa \& Storey, 2020).

The broad targeting nature of miRNAs is illustrated by the fact that diverse animals such as hypoxic squid from the bottom of the ocean (Hadj-Moussa, Logan, Seibel, \& Storey, 2018) all the way to hypoxia-exposed high-altitude birds (X. Chen et al., 2018) rely in part on miRNAs for stress-induced metabolic reorganization. Even humans can coordinate miRNA-mediated responses to the hypoxic insults experienced in high-altitude environments, albeit in a less adaptive manner (Y. Yan et al., 2015). It is interesting to note that a comparative analysis of existing oxygen-responsive miRNAs 'OxymiR' from over 20 evolutionarily distinct animals from diverse environments highlighted the lack of a conserved miRNA response to oxygen-deprivation, and rather the presence of animal and tissue -specific responses (Hadj-Moussa \& Storey, 2020). As for 
naked mole-rats, an initial targeted qPCR-based examination of hypoxia-associated miRNAs in the brains of multiple hypoxia-exposed mole-rat species implicated miRNAs in the modulation of HIF-1 $\alpha$ and other oxygen-sensitive targets discussed herein (Logan et al., 2020).

Building on what we know about naked mole-rat miRNAs, the current study provides the first next-generation sequencing-based post-transcriptional analysis of global miRNA expression in naked mole-rats, where we investigate whole brain responses to normoxic and hypoxic exposures. Previous miRNA work on hypometabolic hypoxia and anoxia tolerant animals guided us to hypothesize that the differentially expressed hypoxic naked mole-rat brain miRNAs will likely be involved in facilitating cytoprotection for successful hypoxic retreats. Our goal was to characterize miRNA mechanisms behind the many adaptive naked mole-rat hypoxia tolerance responses. This was done using a comprehensive bioinformatics platform that included Unix shell applications and custom-built R packages RBioMIR, RBiomirGS, and RBioFS (J. Zhang \& Storey, 2016, 2018), to identify conserved miRNAs in both normoxic and hypoxic naked mole-rat brains. Specifically, we investigated expression profile clustering, differential expression (DE), and gene set (GS) analysis. Understanding the mechanisms responsible for successful hypoxia endurance could prove invaluable at preventing and treating the currently irreversible damage that occurs during stroke, ischemia, and cardiac arrest.

\subsection{Materials and Methods}

\subsection{1 $\quad \underline{\text { Animals }}$}

Eight subordinate and non-breeding naked mole-rat adult males from a group-housed colony at the University of Ottawa (Canada) were used. Refer to Houlahan et al. (2018) for information 
on animal feeding, monitoring, and behaviour. naked mole-rat were randomly divided into two groups $(n=4)$ and placed into two separate experimental chambers with a thin layer of bedding on the floor of each. Chambers were sealed and constantly ventilated with gas mixtures, set to the desired fractional gas composition by calibrated rotameters (Praxair, Mississauga, ON, CA). Inflowing gas was provided at a flow rate of $0.5 \mathrm{~L} / \mathrm{min}$, as assessed by a calibrated mass flow meter (Alicat Scientific, Tucson, AZ, USA). Four normoxic control $\left(20.95 \% \mathrm{O}_{2}, 0.05 \% \mathrm{CO}_{2}\right.$, balance $\mathrm{N}_{2}$ ) animals were euthanized by conscience cervical dislocation, immediately followed by decapitation. Oxygen levels were continuously monitored throughout the experiment using a FC$10 \mathrm{O}_{2}$ analyzer (Sable Systems International, Las Vegas, NV, USA). For hypoxia exposures, incurrent gas was switched to $\left(7 \% \mathrm{O}_{2}, 0.05 \% \mathrm{CO}_{2}\right.$, balance $\left.\mathrm{N}_{2}\right)$ and flow rates were increased to 1 $\mathrm{L} / \mathrm{min}$ for $10 \mathrm{~min}$ to rapidly reduce the $\mathrm{O}_{2}$ content of the chamber to $7 \% \mathrm{O}_{2}$ and then returned to $0.5 \mathrm{~L} / \mathrm{min}$ for the remaining $4 \mathrm{~h}$ of exposure. Hypoxic animals were then euthanized as described for normoxic animals. Whole brains, excluding the brainstem, were rapidly dissected within 30 sec, immediately flash frozen in liquid nitrogen, and stored at $-80{ }^{\circ} \mathrm{C}$ until analysis.

All naked mole-rat experiments complied with the requirements proposed by the Canadian Council on Animal Care and were approved by the University of Ottawa Animal Care Committee. Animal care protocol [\#2535].

\subsection{2 $\underline{\text { RNA isolation }}$}

Total RNA isolation was performed on approximately $50 \mathrm{mg}$ of whole brain tissue, excluding the brainstem ( $n=4$ individual animals) for both normoxic and hypoxic naked mole-rats. RNA was isolated using a Trizol-chloroform (Invitrogen; Cat\#15596-018) extraction as per the manufacturers instruction and as described in Hadj-Moussa et al. (2020). Isolated total RNA pellets were resuspended in $50 \mu \mathrm{L}$ RNase-free water. RNA purity and concentration were assessed using 
a Take3 micro-volume plate and spectrophotometer (BioTek, Winooski, VT, USA) to measure the 260/280 nm ratio. Integrity of the RNA samples was examined by verifying the presence of sharp bands for $28 \mathrm{~S}$ and $18 \mathrm{~S}$ rRNA using gel electrophoresis on a $1 \%$ agarose gel with SYBR Green (Invitrogen; Cat\# S7563).

\subsubsection{Small RNA sequencing}

Small RNA-Sequencing was performed by BC Cancer Genome Sciences Centre (Vancouver, BC, Canada). Prior to library construction, total RNA quality was verified with Qubit and Agilent DNA 1000 Series II systems, only samples with RIN scores $>7$ were used for library construction. Total RNA in $8 \mu \mathrm{L}$ was ligated with $2 \mu \mathrm{L}$ of a $2.5 \mu \mathrm{M}$ 3' DNA adapter (5', NNNNNNTGGAATTCTCGGGTGCCAAGTCG 3', N = randomized sequence) and incubated at $70{ }^{\circ} \mathrm{C}$ for 2 min to denature RNA secondary structure, then snap chilled on ice. Reactions were then combined with $10 \mu \mathrm{L}$ of ligation mix containing truncated T4 RNA ligase $2(200 \mathrm{U} / \mu \mathrm{L}$, New England Biolabs). This was incubated for 1 hour at $22^{\circ} \mathrm{C}$ and purified twice using RNA MagClean DX beads (Aline Biosciences) at room temperature to remove excess adapters. Next, 3' adapterligated RNAs were ligated to heat denatured 5, miRNA adapters (5' UUCCCUACACGACGCUCUUCCGAUCUNNNNNN 3', $\mathrm{N}$ = randomized sequence) using T4 RNA ligase $\left(5 \mathrm{U} / \mu \mathrm{L}\right.$, Ambion). These $20 \mu \mathrm{L}$ reactions were incubated for $60 \mathrm{~min}$ at $37^{\circ} \mathrm{C}$.

For first strand synthesis, the reverse transcriptase (RT) primer (5' CGACTTGGCACCCGAGAATTCCA 3') was incubated with ligated template at $65^{\circ} \mathrm{C}$ for 10 min then snap chilled on ice. Reverse transcription as performed using Maxima H minus RT (200 $\mathrm{U} / \mu \mathrm{L})$ in a $40 \mu \mathrm{L}$ reaction incubated for 60 min at $44^{\circ} \mathrm{C}$. First strand cDNA was purified using an upper and lower bead clean-up (PCRClean DX beads, Aline Biosciences) to remove excess RT primer and reduce non-target products prior to PCR enrichment. Next, 15 cycles of PCR using a 
paired-end

primer

(PE

1.0

5'AATGATACGGCGACCACCGAGATCTACACTCTTTCCCTACACGACGCTCTTCCGAT

$\begin{array}{llll}\text { CT } & \text { 3') } \text { and } & \text { miRNA }\end{array}$

(CAAGCAGAAGACGGCATACGAGATCNNNNNNATCGTACGTCGACTTGGCACCCGA

GAATTCCA; underlined index sequence is unique for up to 96 samples) was performed in a 50

$\mu \mathrm{L}$ reaction volume incorporating Phusion Hot Start high fidelity DNA polymerase

(NEB). Amplified library was loaded onto a 12\% PAGE gel and the region containing the miRNA library ( $\sim 150 \mathrm{bp})$ was manually excised from the gel. The size-selected library was ethanol precipitated and purified. Quality checks of the final library was performed using Qubit and Agilent DNA 1000 Series II assays prior to sequencing on an Illumina NextSeq500 instrument generating single-end 75 base reads.

\subsubsection{Data processing}

The naked mole-rat sequence reads generated have all been deposited to the SRA database where they have been made publicly available using the following accession numbers [SRR12668869 - SRR12668876]. Initial data processing was performed as described in HadjMoussa et al. (2020) using the RBioMIR pipeline developed by (J. Zhang, Hadj-Moussa, \& Storey, 2016) https://github.com/jzhangc/git RBioMIR. Briefly, adapters were trimmed and reads with Phred quality scores $<30$, as determined by FastQC quality reports (http://www.bioinformatics.babraham.ac.uk/projects/fastqc/) were removed using fastq-mcf (Aronesty, 2011). Next, non-miRNA small RNA species (rRNA, tRNA, snRNA, snoRNA, and piRNA) were filtered and removed from the trimmed reads. Bowtie was used to align and filter reads with default settings. A 'mature miRNA positive' reference file was then constructed using all mature miRNA sequences from all species available on miRBase (www.mirbase.org). Filtered 
reads were aligned against miRNA using Bowtie, with seed sequence length set to 20 nucleotides, only perfect seed sequence matches were reported. Mature miRNA read counts were sorted using Samtools software (Li et al., 2009). Read counts per sample were then imported into the R environment (R Core Team, 2017; J. Zhang \& Storey, 2018). The miRNA ortholog with the highest read count was used and only miRNAs with more than four reads were considered for downstream quantification. These filtered read counts were then normalized using the voom method (Law, Chen, Shi, \& Smyth, 2014).

\subsection{5 $\quad$ Unsupervised clustering analysis}

The R package gplots was used to perform unsupervised hierarchical clustering (Warnes et al., 2015), with Ward clustering method [45], https://github.com/jzhangc/git_RBioArray. Clustering was performed on both miRNA and animal samples to generate a heatmap with dendrograms.

Unsupervised principal component analysis (PCA) was used to further characterize miRNA expression profiles for each sample to identify similarities between gene expression patterns and samples (Lenz, Müller, Zenke, \& Schuppert, 2016). PCA analysis works by reducing data dimensionality by linearily transforming data into uncorrelated latent variables (or principal components) that collapse data into a lower dimensional space while retaining maximum data variance (Lenz et al., 2016). Biplots were generated by plotting PC1, PC2, and PC3 (which accounted for the minimum number of PCs that represented the most variance) and superimposing PCA score distributions for miRNAs. 


\subsubsection{Differential expression analysis}

The limma R package was used for miRNA differential expression (DE) analysis. This was conducted by performing statistical tests on miRNA expression through linear model fitting with empirical Bayseian (eBayes) tests (Ritchie et al., 2015). The threshold for statistically significant differentially expressed miRNAs between normoxic and hypoxic animals was set to an FDRadjusted $p<0.01$ and fold change $>1.5$.

\subsubsection{Gene set analysis}

To functionally characterize miRNA expression profiles in hypoxic animals, relative to the normoxic control group, the R package RBiomirGS was used to perform logistic regression-based gene set (GS) analysis (J. Zhang \& Storey, 2018) (https://github.com/jzhangc/git_RBiomirGS). Since naked mole-rat miRNA:mRNA interaction mapping is not available, we used mouse miRNA orthlogos for miRNA:mRNA mapping. The following miRNA:mRNA mapping databases were searched DIANA-microT-CDS, ElMMo, MicroCosm, miRanda, miRDB, PicTar, PITA, and TargetScan (Betel, Wilson, Gabow, Marks, \& Sander, 2007; Friedman, Farh, Burge, \& Bartel, 2008; Gaidatzis, van Nimwegen, Hausser, \& Zavolan, 2007; Garcia et al., 2011; Kertesz, Iovino, Unnerstall, Gaul, \& Segal, 2007; Krek et al., 2005; Lewis, Burge, \& Bartel, 2005; Paraskevopoulou et al., 2013; X. Wang, 2008). Only the top 5\% of predicted miRNA:mRNA interactions were used to ensure high confidence. The identified mRNA targets and miRNA expression information were then converted into potential mRNA regulation scores as described (J. Zhang \& Storey, 2018). The targeted mRNAs and their calculated mRNA scores were then enriched via logistic regression-based gene set enrichment that was performed using the Gene ontology (GO) terms and Kyoto Encyclopedia of Genes and Genomes (KEGG) gene sets (Ashburner et al., 2000; Kanehisa \& Goto, 2000; The Gene Ontology Consortium, 2017). 
Complete miRNA lists were used for GS analysis. The threshold for significantly enriched gene sets was an FDR-adjusted $p$-value $<0.05$ and corresponding coefficients for each KEGG pathway and GO term. KEGG pathways were visualized with miRNA regulatory effects on target genes using R package pathview (Luo \& Brouwer, 2013). Positive model coefficients (green) indicate that negative regulation (inhibition) by microRNA is reduced during hypoxia, relative to control. A negative coefficient (red) indicates that inhibition by miRNAs is increased during hypoxia.

Significantly enriched GO Biological Processes were additionally analyzed using the REVIGO online enrichment tool to summarize GO lists by removing redundant terms (http://revigo.irb.hr/). REVIGO summarized terms were visualized using Cytoscape3.7.2. Nodes are colored by FDR corrected $p$-values, where dark blue corresponds to a smaller $p$-value and colours closer to yellow represent larger, but still significant, FDR corrected $p$-values. The size of nodes corresponds to the number of semantically related GO terms that the node encompasses. The grey connecting edges between nodes represent sematic relationships.

\subsection{8 $\quad$ RNA polyadenylation and cDNA synthesis}

For miRNA analyses, $3 \mu \mathrm{g}$ of total RNA was processed as described by (Biggar, Wu, \& Storey, 2014). Polyadenylation was performed using the Epi-Bio PolyA tailing kit (Epicentre, Madison, WI, USA; Cat\# PAP5104H) as per the manufacturer's instructions. Prior to cDNA synthesis, $10 \mu \mathrm{L}$ polyadenylated RNA samples were combined with $5 \mu \mathrm{L}$ of $250 \mathrm{pM}$ universal stem-loop adapter primer (Supp. Table S1). On a thermocycler, reactions were heated to $95{ }^{\circ} \mathrm{C}$ for $5 \mathrm{~min}$, incubated for $5 \mathrm{~min}$ at $65{ }^{\circ} \mathrm{C}$, then chilled on ice. Reverse transcription was then performed using the M-MLV reverse transcriptase (Invitrogen; Cat\# 28025013) as per the manufacturer's instructions. The following PCR-program was used: $16{ }^{\circ} \mathrm{C}$ for $30 \mathrm{~min}, 42{ }^{\circ} \mathrm{C}$ for $30 \mathrm{~min}$, and $85^{\circ} \mathrm{C}$ for $5 \mathrm{~min}$. 
For mRNA analyses, $1 \mu \mathrm{g}$ of total RNA was combined with $1 \mu \mathrm{L}$ of standard oligo-dT (200 ng/ $\mu \mathrm{L}$ 5'-TTTTTTTTTTTTTTTTTTTTTV-3'; V = A or G or C) in a total volume of $10 \mu \mathrm{L}$ RNasefree water. Reactions were incubated in a thermocycler for $5 \mathrm{~min}$ at $65^{\circ} \mathrm{C}$ then immediately chilled on ice. The same reverse transcription reaction used for miRNA cDNA synthesis was used for mRNA cDNA synthesis. However, the following PCR program was used: $42{ }^{\circ} \mathrm{C}$ for 60 min.

\subsection{9 $\quad$ RT-qPCR amplification of miRNA and $m R N A$}

For miRNA analyses, Forward microRNA-specific primers were designed from the naked mole-rat brain transcriptomic data and the universal reverse primer was designed using the described method (Supp. Table S1) (Biggar et al., 2014). MicroRNA RT-qPCR reactions were prepared as described by (Hoyeck, Hadj-Moussa, \& Storey, 2019) and run on a CFX Connect ${ }^{\mathrm{TM}}$ Real-Time PCR Detection System (BioRad, Cat \#1855201) following MIQE guidelines (Bustin et al., 2009). The following PCR program was used for miRNAs: $3 \mathrm{~min}$ at $95{ }^{\circ} \mathrm{C}$, followed by 60 cycles of $95^{\circ} \mathrm{C}$ for $10 \mathrm{sec}$ and $60^{\circ} \mathrm{C}$ for $30 \mathrm{sec}$.

For mRNA analyses, forward and reverse primers were designed using the NCBI Primer Blast tool on the H. glaber genome (taxid: 10181). Target mRNA and reference gene primers are listed in (Supp. Table S1). Primers generated by Integrated DNA Technologies were designed for the following genes: GLUT5, KHK, LDH, and SREBP2. Primer specificity was verified using a postPCR run melt-curve analysis. For mRNA RT-qPCR, the same reaction mixture described above was used and run with the following program; incubation for 3 min at $95^{\circ} \mathrm{C}$, followed by 49 cycles of $95^{\circ} \mathrm{C}$ for $10 \mathrm{sec}, 57^{\circ} \mathrm{C}$ for $20 \mathrm{sec}$, and $72^{\circ} \mathrm{C}$ for $20 \mathrm{sec}$. 


\subsubsection{Quantification of $R T-q P C R$ and statistics}

Differential gene expression levels for miRNA and mRNA $(n=4)$ were calculated using the $\Delta \Delta \mathrm{Cq}$ comparative method. Cycle threshold $(\mathrm{Cq})$ values were transformed to the $2^{-\mathrm{Cq}}$ form, to allow for the normalization of the miRNA or mRNA of interest to the endogenous controls (Andersen, Jensen, \& Ørntoft, 2004; Peltier \& Latham, 2008; Schmittgen \& Livak, 2008). For miRNA quantification, Snord66 was found to be the most stable endogenous control gene between normoxic and hypoxic conditions (Logan et al., 2020). For mRNA relative quantification, GAPDH was experimentally determined to be a suitable reference gene and was stably expressed in both normoxic and hypoxic naked mole-rat brains. Data was collected and analyzed as mean relative expression (mean \pm SEM, where $n=4$ independent biological replicates from different animals at each sampling point, run in triplicate assays).

Statistical data analysis of miRNA and mRNA relative expression between normoxic and hypoxic conditions was considered significant (indicated by *) when a Student's t-test resulted in $p<0.05$ using the RBioplot statistical and graphing $\mathrm{R}$ package (J. Zhang \& Storey, 2016) (https://www.kenstoreylab.com/research-tools/rbioplot/).

\subsubsection{Total protein isolation}

Total protein extracts were prepared for immunoblotting as previously described (HadjMoussa \& Storey, 2018). Briefly, $\sim 50 \mathrm{mg}$ of brain tissue ( $n=4$ individual animals) was homogenized in 1:2 w:v chilled homogenization buffer using a Polytron PT10 homogenizer. Samples were then centrifuged at $4{ }^{\circ} \mathrm{C}$ for $15 \mathrm{~min}$ at $10,000 \mathrm{~g}$, and supernatants containing soluble proteins were collected. Protein concentrations were measured using the BioRad reagent (Cat\# 5000002; Hercules, CA) as per the manufacturer's instructions. Soluble brain protein concentrations were standardized using homogenization buffer and mixed 1:1 v:v with SDS buffer 
and $10 \% \beta$-mercaptoethanol. Samples were then boiled for $10 \mathrm{~min}$ and subsequently stored at $20{ }^{\circ} \mathrm{C}$ until use.

\subsubsection{Western blotting, protein quantification, and analysis}

Equal amounts of total protein homogenates (15-40 $\mu \mathrm{g}$ depending on target) of each sample were loaded onto 10-12\% polyacrylamide gels and prepared with 5\% upper stacking gels. SDSPAGE and immunoblotting followed the protocol previously described in (Hadj-Moussa \& Storey, 2018). Briefly, proteins were separated in SDS-PAGE running buffer at $180 \mathrm{~V}$ for $60-120$ min at $4{ }^{\circ} \mathrm{C}$ with a BioRad Mini Protean III system. Protein gels were then electroblotted by wet transfer onto a $0.45-\mu \mathrm{m}$ polyvinylidene difluoride membrane in transfer solution at $4{ }^{\circ} \mathrm{C}$ for $1-16 \mathrm{~h}$ at 160 $\mathrm{mA}$. Transferred membranes were air-dried for $15 \mathrm{~min}$, reactivated in methanol for $5 \mathrm{~min}$, and incubated in $2-10 \% \mathrm{v} / \mathrm{v}$ skim milk in TBST, and blocked for $10-45 \mathrm{~min}$ in $2-10 \% \mathrm{w}: \mathrm{v}$ milk in TBST. This was followed by $3 \times 5$ min washes with TBST and overnight incubation with the appropriate primary antibody at 1:1000 v:v dilution in TBST at $4{ }^{\circ} \mathrm{C}$.

The following primary antibodies were used; GLUT5/ SLC2A5 (AbClonal; Cat\# A13650), KHK (AbClonal; Cat\# A7440), LDH (Applied Biological Materials Inc.; Cat\# Y104790), and SREBP2/ SREBF2 (AbClonal; Cat\# A13049). After probing with primary antibody, membranes were washed for $3 \times 5$ min with TBST and incubated with HRP-linked anti-rabbit, mouse, or goat secondary antibody (BioShop; 1:8000 v:v dilution in TBST) for $30 \mathrm{~min}$ at room temperature. Membranes were then washed in TBST and bands were visualized with enhanced chemiluminescence $\left(\mathrm{H}_{2} \mathrm{O}_{2}\right.$ and Luminol) on a ChemiGenius Bio-Imaging System (Syngene, Frederick, MD). Membranes were then stained with Coomassie blue $(0.25 \% \mathrm{w} / \mathrm{v}$ Coomassie brilliant blue, $7.5 \% \mathrm{v}: \mathrm{v}$ acetic acid, and $50 \% \mathrm{v}: \mathrm{v}$ methanol) to visualize total protein levels. 
To quantify relative protein abundance, chemiluminescent bands were quantified by densitometry using GeneTools Software (Syngene, MD, USA). To correct for minor variations in sample loading, the band intensity in each lane was standardized against a group of Coomassie blue-stained protein bands. Immunoblot data are expressed as mean $\pm \mathrm{SEM}$, relative to control values ( $n=4$ from different animals). Data were statistically analyzed using a Student's t-test, $p<$ 0.05 represented statistically significant results. Statistical analyses were performed using the RBioPlot statistics and graphing R package (J. Zhang \& Storey, 2016).

\subsection{Results}

\subsubsection{Small RNA-seq analysis and identification of conserved miRNAs}

A total of 94,131,373 reads were identified in normoxic and 93,648,727 reads were identified in hypoxic samples. Data processing steps (adapter trimming, low quality read removal, short read removal, non-miRNA small RNA removal) reduced normoxic naked mole-rat brain reads to $57,208,480$ reads ( $61 \%$ of total) and hypoxic brains to $55,129,380$ reads ( $59 \%$ of total). Remaining reads were mapped to the miRNA database obtained from miRBase- normoxic samples had $46,862,411$ reads ( $82 \%$ of pre-processed reads) that aligned to mature miRNAs and hypoxic samples had $44,063,710$ reads ( $80 \%$ of pre-processed reads) that mapped to mature miRNAs. Overall, 212 conserved mature miRNAs were identified in naked mole-rat brains.

\subsubsection{MiRNA differential expression analysis}

From the 212 conserved mature miRNAs identified, 18 miRNAs ( $\sim 8 \%$ of conserved miRNAs) were differentially expressed in hypoxic naked mole-rats, as compared to normoxic naked molerat brains (Figure. 4.1). Hypoxic naked mole-rats displayed 6 significantly upregulated, and 12 
significantly downregulated miRNAs (FDR adjusted $p<0.05$ and fold change $>1.5$ ). Significantly upregulated miRNAs include: miR-365a/b-3p, miR-383-5p, miR-935-3p, miR-155-5p, miR-1323p, miR-184 (Fig. 1). Significantly downregulated miRNAs include: miR-376b-3p, miR-889-3p, miR-127-5p, miR-381-3p, miR-379-5p, miR-136-3p, miR-301a-3p, miR-335-5p, miR-181c-5p, miR-1-3p, miR-101-3p, miR-19b-3p (Figure 4.1). Complete miRNA DE analysis results are listed in Supp. Table S2.

\subsection{3 $\quad$ RT-qPCR validation of RNA-seq analysis}

Levels of a subset of four randomly selected DE miRNAs were validated with RT-qPCR. The randomly selected DE miRNAs displayed the same expression trends in both RNA-Seq analysis and RT-qPCR, demonstrating a consistency between RNA-Seq DE and RT-qPCR validations (Figures 4.1 and 4.2). The following three miRNAs (miR-132-3p, miR-184-3p, and miR-383-5p) were measured and found to be significantly upregulated and levels of miR-301a-3p were downregulated during hypoxia (Figure 4.2).

\subsubsection{Cluster analysis}

Unsupervised hierarchical clustering analysis of miRNAs that grouped high, intermediate, and low abundance miRNA expression, displayed an expression pattern difference between normoxic and hypoxic samples (Figure 4.3A). Overall, normoxic samples had more abundant miRNAs, while hypoxic samples had a larger group of less abundant miRNAs. Samples did not group into their experimental conditions, as Normoxic brain 3 was grouped separately from the other miRNAs due to its visibly different pattern of miRNA expression (Figure 4.3A). However, it should be noted that normoxic samples displayed similar miRNA expressions to each other, except for Normoxic 3, while hypoxic samples also displayed a similar global trend. Indeed, when PCA 
results are examined, we see a clear separation between normoxic and hypoxic samples on PC1 and PC3, as well as on PC2 and PC3 (Figure 4.3).

\subsubsection{Gene set analysis}

Mapped miRNA:mRNA interactions predicted on all differentially expressed miRNAs are reported in (Supp. Table S3). In this study, KEGG pathways as well as GO Molecular Function, Cellular Component, and Biological Process terms were assessed for GS analysis. Significantly hypoxia-enriched KEGG pathways included a total of 9 pathways: Cytokine-cytokine receptor interaction lacked miRNA-inhibition while the remaining 8 pathways Ribosome, aminoacyl tRNA biosynthesis, proteasome, valine leucine and isoleucine biosynthesis, glycolysis gluconeogenesis, pyruvate metabolism, viral myocarditis and Parkinson's disease (Figure 4.4A). The complete list for KEGG GS analysis can be viewed in (Supp. Table S4).

Of the 28 hypoxia-enriched GO Molecular Function terms, 13 were miRNA-inhibited (orange) including RNA binding terms, translational processes such as elongation and initiation, microfilament motor activity, and structural constituents of nuclear pores, and ligase activity forming carbon oxygen bonds (Figure 4.4B). The 13 GO Molecular Function terms that lacked miRNA inhibition (blue) included serine and threonine kinase and phosphatase activity, TGF $\beta$ and activin binding, and transcriptional coactivator binding (Figure 4.4B). The complete list for GO Molecular Function terms can be viewed in (Supp. Table S5).

Enriched GO Cellular Component terms that were inhibited by hypoxia-responsive miRNAs (orange) were focused on ribosomal components, mitochondrial parts, and proteasomal machinery

(Figure 4.4C). While RNAi effector complexes and apical cellular regions appeared to lack 
hypoxia-mediated inhibition (blue) (Figure 4.4C). The complete list for GO Cellular Component analysis can be viewed in (Supp. Table S6).

GS analysis identified 200 GO Biological Processes enriched in hypoxia-exposed brains, this included 118 terms that lacked miRNA inhibition (blue) and 82 that were predicted to be significantly miRNA-inhibited (orange) (Figure 4.4D). REVIGO analyzed GO Biological Processes enrichment resulted in five distinct semantically related networks with $\geq 3$ members (Figure 4.5). The first network had 23 members related to RNA metabolism, external responses to stress stimuli, and tissue development (Figure 4.5). The second largest network (22 members) was related to signal transduction and transcription factor pathways, neuronal processes, and regulation of post-transcriptional silencing and post-translational modification contained the most sematic relationships (Figure 4.5B). The third network ( 8 members) was related to small ion and amino acid transport and localization (Figure 4.5C). The fourth network comprised of only 3 members was related to ribosomal biogenesis and histone ubiquitination (Figure 4.5D), and the fifth network, also with 3 members, was related to lipid biosynthesis and remodelling (Figure 4.5E). Complete GS analysis of GO Biological Process terms are listed in (Supp. Tables S7).

\subsubsection{Transcript mRNA levels of downstream targets}

The mRNA targets examined were selected based on previously experimentally validated miRNA:mRNA target predictions of carbohydrate metabolism genes under the regulation of hypoxia-responsive naked mole-rat brain miRNAs (Figures 4.6A and 4.6C). Transcript levels of $k h k$ and srebp 2 were unaffected by hypoxic exposures (Figure 4.6A). Levels of both glut 5 and $l d h$ were significantly elevated during hypoxia with an increase of 3.34 -fold and 1.38 -fold, respectively (Figure 4.6A). 


\subsubsection{Protein expression levels of downstream miRNA targets}

Protein expression levels were assessed using immunoblotting where relative expression levels of KHK and GLUT5 were found to decrease by 0.51 -fold and 0.78 -fold, respectively (Figure 4.6B). LDH protein levels were hypoxia upregulated by 1.41-fold (Figure 4.6B). Protein levels of SREBP2 were downregulated during hypoxia by 0.59 -fold (Figure 4.6B).

\subsection{Discussion}

\subsubsection{Overall miRNA trends during hypoxia}

Few mammals have evolved the unique and unusual adaptions that naked mole-rats posses, and currently, many of the mechanisms behind these adaptations remain a mystery. At first glance, the hypoxia-responsive miRNA species and pathways being targeted in hypoxic naked mole-rat brains appear to promote protection and hypometabolism. The main miRNA-mediated hypoxia tolerance mechanisms we identified in naked mole-rat brains are as follows: [1] the inhibition of non-essential energy-expensive processes including protein translation, [2] the regulation of anaerobic fructose-based glycolysis, [3] a promotion of neuroprotective functions, and [4] the induction of signal transcription pathways to modulate select gene activation.

Global whole brain analysis identified 212 conserved miRNAs from both normoxic and hypoxic naked mole-rats, of which 18 were significantly differentially expressed during hypoxia, approximately $8 \%$ of total identified miRNAs (Figure 4.1). While the unsupervised hierarchical heatmap did not show a clear separation in overall miRNA expression profiles between normoxic and hypoxic naked mole-rat brains (Figure 4.2A), subsequent PCA analysis clearly separated normoxic and hypoxic samples along the PC1 and PC3 axes, collectively accounting for $59.51 \%$ 
of principle components (Figure 4.2B). The PCA plot also shows that hypoxic naked mole-rats are more closely clustered together as compared to normoxic animals, suggesting more of a stressspecific response, rather than random individual animal-specific response (Figure 4.2B). Below we explore some of the miRNA targeted KEGG and GO pathways implicated in hypoxia and begin with the discussion of energy preservation.

\subsubsection{Hypoxia-mediated miRNA inhibition of non-essential energy expensive processes}

\subsubsection{Protein translation}

The inhibition of non-essential energy-expensive processes such as de novo protein synthesis was predicted to be heavily miRNA-suppressed during hypoxia. Hypoxia-responsive miRNAs targeted various KEGG (inhibition of aminoacyl tRNA biosynthesis) and GO CC terms associated with ribosomal structural subunits, ribonucleoprotein complexes, translation preinitiation, cytosolic localization, binding, and functions are miRNA-inhibited during hypoxia (Figures 4.4A and 4.4C). This is in addition to the inhibition of GO MF terms involved in translation factor activity RNA binding, aminoacyl tRNA editing, and tRNA binding (Figure 4.4B). For example, hypoxia-upregulated levels of miR-132 have been shown to suppress transcription of both small and large ribosomal proteins (Hewitson et al., 2019). MiR-132 can bind to conserved binding sites on transcriptional regulators including $\mathrm{p} 300$. This is required for the activity of Sp1, YY1, and CREB and for the transcription of ribosomal genes, and factors that interact with the TATAbinding protein (Hewitson et al., 2019; Lagos et al., 2010). Another direct example of miRNA involvement in ribosomal regulation is the targeting of 5S rRNA ribosomal protein by the hypoxiaupregulated miR-383 (Xinyu Wang et al., 2015). The suppression of protein translation in hypoxic naked mole-rats is supported by a recent multi-tissue study of the Akt/mTOR pathway and key downstream protein translation factors that demonstrated an attenuation of protein synthesis in 
hypoxic naked mole-rat muscle, lung, and brain (Al-attar, Childers, Nguyen, Pamenter, \& Storey, 2020).

In addition to the apparent targeting of protein synthesis, another aspect of protein turnover that was targeted during hypoxia was inhibition of the KEGG 'proteasome' term and the GO CC 'proteasome complex' term (Figure 4.4C). This hypoxia-mediated regulation of proteolytic machinery could provide a mechanism for energy-saving and cellular proteome stabilization during stress. However, novel naked mole-rat cytosolic factors have been found to promote enhanced proteasome activity (Rodriguez et al., 2014). Additionally, naked mole-rats express high cytosolic heat shock protein levels as compared to mice, and also increase ATP-independent heat shock proteins during acute hypoxia (Nguyen, Deck, \& Pamenter, 2019; Rodriguez et al., 2014).

\subsubsection{Cellular proliferation}

Another way that these changing miRNAs appear to be inhibiting anabolic processes and cellular growth is by targeting cellular proliferation factors. Take for example the hypoxiaupregulated miR-132 that has been reported to suppress cell proliferation by inhibiting FOXA1 (D. Wang, Ren, Ren, Fu, \& Yu, 2018), modulating the Notch-Hes1 pathway (D. Chen et al., 2 018), and targeting FOXP2 (Clovis, Enard, Marinaro, Huttner, \& De Pietri Tonelli, 2012). Interestingly, miR-132 is reported to be an evolutionarily conserved stress-responsive miRNA involved in the co-regulation of inflammatory, metabolic, and neuronal growth pathways (Haviv, Oz, \& Soreq, 2018), see below for our discussion of its neuronal functions.

Additionally, the over-expression of miRNA-365 and miR-383 have been experimentallyvalidated to target Paired box protein Pax-6 (PAX6), promoting the inhibition of cellular proliferation (F. Yan, Tu, Duan, Wang, \& Lin, 2018). PAX6 is a transcription factor that targets 
neuronal proliferation, development, and the maintenance of adult brains (Yogarajah et al., 2016). When adult rat brains were exposed to ischemic strokes, the overexpression of miR-365 that suppressed PAX6, contributed to a reduction in neurogenesis, an energetically-expensive process that, in the case of hypoxic naked mole-rats, may be repressed during periods of oxygen limitation and hypometabolism (Mo et al., 2018). Other specific targets of the hypoxia-upregulated miRNAs that could also be contributing to inhibited proliferation include miR-383 targeting of a proliferating-inducing ligand (APRIL), the tripartite motif 27 protein (TRIM27), and Cyclin D1 (Cui, Chen, Yao, Zhang, \& Ding, 2018; J. Jiang, Xie, Liu, Shi, \& Chen, 2019; Zhou Xu et al., 2014). Where the suppression of cyclin D1, a protein required for the progression of the cell cycle from the $\mathrm{G} 1$ phase, is likely contributing to cell cycle $\mathrm{G}_{0} / \mathrm{G}_{1}$ arrest (Zhou Xu et al., 2014).

\subsubsection{Switching fuel sources during hypoxia}

The active regulation of anaerobic metabolism has been characterized in the brains of hypoxiatolerant organisms (Boutilier, 2001). Our study is the first to suggest a miRNA-mediated mechanism supporting this metabolic reorganization in naked mole-rats. Hypoxic naked mole-rat brains have been shown to drastically suppress mitochondrial oxidation, switching from a mixed carbohydrate/lipid energy profile to a strictly carbohydrate-centered state (Pamenter et al., 2019). Investigations on briefly anoxic naked mole-rats demonstrated the activation of a fructose-driven anaerobic glycolytic pathway for energy production during oxygen-deprivation (Park et al., 2017). Other studies have also confirmed the possibility of fructose metabolism in specific brain regions (Oppelt, Zhang, \& Tolan, 2017), which lead to our investigation of the potential involvement of miRNAs in regulating this activation during hypoxia. Our findings show that miRNAs may be contributing to the naked mole-rat metabolic shift via targeting and inhibiting key metabolic KEGG terms including the KEGG glycolysis and gluconeogenesis pathways and the pyruvate 
metabolism pathway (Figure 4.4A). Enriched GO CC terms including 'mitochondrion' and 'mitochondrial protein complex' were predicted to be miRNA-inhibited, supporting a shift away from mitochondria-centered $\beta$-oxidation, a prediction that has been experimentally validated (Figure 4.4C) (Pamenter et al., 2019).

Many of the hypoxia-responsive miRNAs we identified were predicted by TargetScan and KEGG to target genes involved fructose metabolism. However, the seven fructose and glycolysis targeting miRNAs (miR-365a/b-3p, miR-136-3p, miR-301a-3p, miR-155-5p, miR-355-5p, miR935, and miR-383) appeared to be non-directional as their involvement did not indicate an overall activation or inhibition of fructose metabolism (Figure 4.7). Take for example, hypoxiaupregulated miR-155-5p, it indirectly promotes hexokinase 2 (HK2) transcription by activating the signal transducer and activator of transcription 3 (STAT3) that binds to the HK2 promoter thereby inducting its transcription (S. Jiang et al., 2010) (Figure 4.7). Hexokinase catalyzes the first committed step in glycolysis by phosphorylating glucose and increased miR-155-5p suggests its induction during hypoxia. Ketohexokinase (KHK) is another enzyme in the fructose metabolism pathway that was targeted in naked mole-rat brains by hypoxia-upregulated miR-365a/b-3p. KHK is responsible for phosphorylating fructose into fructose-1-phosphate in the first step of fructose glycolytic metabolism. During hypoxia, mRNA levels of KHK were unaffected but protein levels were downregulated, suggesting possible miR-365a/b-3p inhibition and translation suppression of KHK transcripts (Figure 4.6).

Despite a lack of direct targeting by the subset of hypoxia-responsive miRNAs, we examined the effects of hypoxia on mRNA and proteins levels of GLUT5, the glucose transporter that shuttles fructose into the cell. While levels of glut 5 mRNA transcripts were hypoxia-upregulated, GLUT5 relative proteins levels were downregulated in hypoxic brains, suggesting a role for post- 
transcriptional regulation either via miRNAs not identified in this study or other mechanisms (Figure 4.6). Our findings in hypoxic brains contradict findings from the Park et al. 2017 study that reported increased KHK mRNA transcript isoforms in acutely anoxic naked mole-rat brains, as well as increased GLUT5 mRNA and protein levels (Park et al., 2017). This suggests that hypoxic naked mole-rats do not rely as heavily on fructose-based anaerobic glycolysis as anoxic naked mole-rat brains.

We also examined hypoxia-induced miRNA targeting on lactate dehydrogenase (LDH), the enzyme necessary for continued anaerobic glycolysis and replenishing NADH reducing potentials during oxygen limitation, regardless of whether fructose or glucose are used as the initial input carbohydrate (Rogatzki, Ferguson, Goodwin, \& Gladden, 2015). During hypoxia, upregulated levels of miR-383-3p and KEGG identified miR-935 were predicted to target and inhibit LDH translation (Fang et al., 2017); however, both mRNA and protein levels of LDH were elevated during hypoxia (Figure 4.6). The increase of LDH mRNA and protein contradict the upregulation of LDH inhibitory miRNAs (miR-383-3p and miR-935-3p) (Figure 4.6). A look at anoxic naked mole-rat brains also shows increased levels of lactate, relative to normoxia, which suggests that it is unlikely that LDH activity would be inhibited during hypoxia and supports the reliance of naked mole-rat brains on anaerobic glycolysis (Park et al., 2017).

Downregulated miR-101-3p levels during hypoxia have also been found to be involved in modulating glucose metabolism, via the direct targeting of TP53-inducible glycolysis and apoptosis regulator (TIGAR) (Fang et al., 2017). While this requires future validation, the lack of suppression on TIGAR could be promoting the metabolic depression observed in naked mole-rat brains by possibly lowering levels of fructose-2,6-bisphosphate and glucose-6-phosphate dehydrogenase, resulting in a decrease of both glycolytic rate and intracellular reactive oxygen 
species (Zhou et al., 2019). Another function that increased TIGAR levels could be promoting is an enhanced capacity for DNA repair by the induction of the pentose phosphate pathway and promotion of anti-apoptotic effects (Bensaad et al., 2006). All of which would be beneficial for hypoxic naked mole-rat brains.

Furthermore, when we compare the hypoxia-induced miRNAs identified in this study with miRNAs upregulated in response to a high-fructose diet, we find that two of these upregulated miRNAs, miR-19b-3p and miR-101-3p, are downregulated in hypoxic naked mole-rat brains (Sud et al., 2017). While the function this imparts on naked mole-rats warrants future research, we can see that SREBP2, a regulator of mainly cholesterol but also fatty acid synthesis, is a downstream target for these miRNAs (Larson \& Park, 2009). During hypoxia, reducing SREBP2 levels makes metabolic sense as naked mole-rat brains switch to carbohydrate catabolism rather than lipid anabolism in their oxygen-limited state. While mRNA levels of SREBP2 remained constant during hypoxia, SREBP2 protein levels were downregulated in hypoxic naked mole-rats, a reduction possibly facilitated by miRNA inhibition (Figure 4.6).

Collectively, these glycolytic and fructose metabolism-related miRNAs illustrate a complex regulatory landscape of carbohydrate metabolism in hypoxic brains. While many of the miRNAs appear to stimulate glycolysis during hypoxia, others appear to be dampening key steps in the pathway. In doing so, they could possibly be acting to coordinate an overall decrease in glycolysis while also facilitating a shift away from lipid breakdown to focus on the use of carbohydrates when oxygen levels are limited. 


\subsubsection{Neuroprotection or neuro-vulnerability?}

\subsubsection{Neuroprotection or neuro-vulnerability?}

With much of current naked mole-rat research focusing on ageing, longevity, and neurophysiology, it was interesting to see that a subset of the targeted processes were promoting neuroprotective functions. Brain-specific enriched targets observed during hypoxia include the GO MF 'Activin binding' term that is a member of the 'transforming growth factor- $\beta$ binding' (TGF $\beta$ ) pathway, both of which significantly lacked miRNA inhibition. Activin A is a neuronal survival factor that was found to rapidly increase after both transient cerebral ischemia and after stroke where it protected brains from oxidative stress (Brackmann et al., 2013; Florio, Gazzolo, Luisi, \& Petraglia, 2007; Mukerji et al., 2007). Therefore, miRNA targeting to promote Activin activity in hypoxic naked mole-rat brains could serve a protective role. The predicted activation of TGF $\beta$ signalling was also observed in the following GO BP terms that lacked miRNA-inhibition: 'Response to transforming growth factor beta', 'Regulation of transforming growth factor beta production', and 'Pathway restricted Smad protein phosphorylation' (Figure 4.4B). Other members of the TGF $\beta$ signal transduction pathway were also predicted to lack miRNA inhibition, such as the targeting of hypoxia-downregulated miR-376b-3p to TGF $\beta 1$, that was previously shown to be downregulated in hypoxic rat brains (Truettner, Katyshev, Esen-Bilgin, Dietrich, \& Dore-Duffy, 2013).

Additional examples of miRNAs that directly modulate adaptive neuronal defenses during hypoxic exposures include key targets of miR-132 and miR-184. Upregulation of miR-132 is known to play a significant role in synaptic function and plasticity (Remenyi et al., 2013) and it has been implicated in memory (Hansen et al., 2016). Mir-132 overexpression in hypoxic naked mole-rat brains could also act to protect against possible tau aggregation, as its deletion is known 
to induce tau accumulation (Hadar et al., 2018). Furthermore, hypoxia-induced upregulation of miR-132 has been reported in Schwann cells where it promotes migration after sciatic nerve injuries (Yao et al., 2016). The hypoxia-upregulation of miR-184 in naked mole-rat brains could also serve neuroprotective functions, as miR-184 regulates survival factor Akt2 and promotes neurogenesis required for brain stress tolerance (Quinlan \& Jimenez-Mateos, 2016). Although levels of Akt2 have yet to be examined in naked mole-rat brains, phosphorylated levels of $\mathrm{Akt} / \mathrm{mTOR}$ proteins in naked mole-rat brains largely remained constant during hypoxia (Al-attar et al., 2020).

During hypoxia, levels of miR-181c-5p were found to decrease in naked mole-rat brains, which has been associated in other studies with oxygen-glucose deprivation (Ge et al., 2019). However, the downstream effects of this warrant further investigation as some studies have found that downregulated miR-181c levels help mitigate hypoxia/ reoxygenation by mitigating apoptosis (Ge et al., 2019), while others have reported that it is the overexpression of miR-181c, not its downregulation, that mediates protection from neuronal apoptosis (L. Zhang, Dong, Li, Hong, \& Wei, 2012). Interestingly, apoptosis is also central to two other enriched gene sets that were predicted to be inhibited by hypoxia-responsive miRNAs in naked mole-rat brains, the KEGG 'Parkinson's disease' pathway as well as the GO CC 'Myelin sheath' term (Figure 4.4A and 4.4C).

\subsubsection{Neuroinflammation}

Three of the hypoxia-responsive miRNAs were also implicated in neuroinflammatory processes. While discussion above highlights the role of miR-155 upregulation during hypoxia as a positive regulator of metabolic processes, it could also be propagating neuroinflammation (Henry et al., 2019). MiR-155 has been associated with enhanced pro-inflammatory responses to alpha- 
synuclein and propagation of neurodegeneration (Thome, Harms, Volpicelli-Daley, \& Standaert, 2016). The degree to which miR-155 propagates neuroinflammation in naked mole-rats remains unknown but it is likely not detrimental as naked mole-rats are known to use robust antineurodegenerative mechanisms to combat hypoxic stressors (Smith et al., 2015). In contrast, miR377 was found to be associated with protecting against neuroinflammation. Reduced levels of miR377 , as observed in hypoxic naked mole-rats, have been shown to contribute to decreased cerebral inflammation, thereby lessening ischemic brain injury (Fan et al., 2018).

\subsubsection{Signal transduction regulated by hypoxia-induced miRNAs}

\subsubsection{HIF-1 $\alpha$ induction by miRNAs}

The HIF-1 $\alpha$ transcription factor has been well-characterized in hypoxic naked mole-rats. Its increased transcript and protein levels and unique sequence relieve it from constant degradation and allow it to adapt to chronic low oxygen availability (Hawkins et al., 2019; Kim et al., 2011). During normoxia, HIF-1 $\alpha$ is constitutively expressed and degraded, until hypoxia stabilizes and increases HIF-1 $\alpha$ activity, which in turn induces the transcription of over 100 downstream genes involved in cell cycle progression, glucose metabolism, and many other processes required for surviving oxygen limitation (Cimmino et al., 2019; L \& T, 2016; Masoud \& Li, 2015). Indeed, the activation of HIF-1 $\alpha$ appears to be a conserved response that has been reported in other hypometabolic and hypoxia/anoxia-tolerant organisms (English, Hadj-Moussa, \& Storey, 2018; Gorr, 2004; Logan et al., 2020; Maistrovski, Biggar, \& Storey, 2012; Morin, McMullen, \& Storey, 2005).

MiR-155 is known to be upregulated during hypoxia where it acts to inhibit HIF-1 $\alpha$, with HIF$1 \alpha$ being able to positively feedback and induce the transcription of more miR-155 (Bruning et al., 2011; Yang, Wang, Xiao, Zhou, \& Shi, 2016). The measured upregulation of HIF-1 $\alpha$ protein levels 
in naked mole-rat brains along with elevated levels of miR-155 suggest that inhibitory effects of miR-155 on HIF-1 $\alpha$ protein levels are less pronounced in hypoxic naked mole-rat brains (Bruning et al., 2011) (Figure 4.1). Another direct regulator of HIF-1 $\alpha$ is miR-335 that displayed reduced expression in hypoxic naked mole-rat brains, likely contributing to the overexpression of HIF-1 $\alpha$ in hypoxic naked mole-rat brains (Liu et al., 2015) (Figure 4.1). However, it should be noted that while the downregulation of miR-335 is beneficial 24 hours after a stroke, its upregulation is beneficial immediately after stroke induction (Liu et al., 2015). An indirect example of HIF-1 $\alpha$ miRNA regulation is miR-101 and its targeting of VHL, a HIF-1 $\alpha$ negative regulator under normoxic conditions (N. Liu et al., 2016) (Figure 4.1). The hypoxia-induced downregulation of miR-101 in naked mole-rat brains could be due to the fact that VHL is downregulated by other means during hypoxia, and as such it is possible that the cell does not need to expend additional energy in inhibiting VHL and promoting HIF-1 $\alpha$ (Haase, 2006).

\subsubsection{NF $\quad N B$ regulation by miRNAs}

Select central signal transduction pathways such as TGF $\beta$ signalling were predicted to lack miRNA-inhibition in hypoxic naked mole-rat brains. Analysis of a panel of stress-survival proteins performed on naked mole-rat brains found increased protein levels of NFKB and HIF-1 $\alpha$ during hypoxia, suggesting that their involvement in cytoprotective functions (Hawkins et al., 2019). $\mathrm{NF \kappa B}$ is considered a first molecular responder to oxidative stress - where it can rapidly modulate apoptosis, antioxidant defenses, angiogenesis, DNA damage repair, and inflammatory responses (D'Ignazio \& Rocha, 2016; Hayden \& Ghosh, 2012). A possible miRNA-mediated mechanism for the observed upregulation of NFKB in naked mole-rat brains is via the hypoxia-upregulation of miR-365, a known transcriptional activator of NFkB (Figure 4.1) (Zheng Xu et al., 2011). The hypoxia-induced over-expression of miR-155 that targets HIF-1 $\alpha$ in naked mole-rat brains can also 
induce NFאB expression. Once upregulated, NFkB itself can positively feedback to strongly induce the transcription of miR-155 (Figure 4.1) (Mehta \& Baltimore, 2016).

\subsubsection{A comparison of hypoxia-responsive miRNAs}

The findings reported herein corroborate the main conclusions of our recent comprehensive review of oxygen-responsive miRNAs 'OxymiRs' from over 20 diverse species. In that review, we reported that differentially expressed miRNAs seem to be regulating many of the same processes, despite the absence of a single conserved subset of hypoxia-responsive miRNAs being regulated (Hadj-Moussa \& Storey, 2020). Another global observation was that species that are the least hypoxia-tolerant tend to present the most dynamic miRNA response, suggesting that these animals are likely mounting a 'panic' when deprived of oxygen rather than having evolved an organized miRNA mechanism (Riggs et al., 2018).

A study of six South African mole-rat species, including naked mole-rats, used RT-qPCR to measure the expression of 27 previously hypoxia-associated miRNAs in hypoxic and normoxic brains and found these miRNAs to be involved in the inhibition of apoptosis and regulation of neuroinflammation (Logan et al., 2020). It should be noted that not all of the changing miRNAs previously identified in naked mole-rat brains matched the findings of our current study. For example, some of the significantly expressed miRNAs were not identified in our dataset such as hypoxia-upregulated miR-24-5p and miR-592-5p, possibly due to their low abundance (Logan et al., 2020). While miRNAs reported as significantly changing in hypoxic naked mole-rats by Logan and colleagues appeared to share the same general up or down trend, including hypoxiadownregulated miR-26b-5p and miR-126a-5p, but were non-significantly downregulated in our current study (Logan et al., 2020). Possible explanations for this disparity include the individual differences of the animals used in the study that might have been compounded by the relatively 
limiting sample size of four. It was surprising that miR-210-5p, a miRNA that was downregulated in Logan et al.'s study as well as in various other models of oxygen deprivation where it acts to stabilize HIF-1 $\alpha$ (Bavelloni et al., 2017; Logan et al., 2020), was not identified in our RNA-Seq dataset.

A look beyond mole-rats shows similarities between naked mole-rat miRNA expression and the expression of other hypoxia-tolerant organisms including the downregulation of miR-101 that was also observed in hypoxic Tibetan highland pigs (Sus scrofa) that live in chronically hypoxic elevations between 2500-4300 m above sea level (B. Zhang et al., 2015). If we look to hypoxic birds at high-altitudes, we see that miR-19b-3p is downregulated both in the brains of our hypoxic naked mole-rats and in the Great tit (Parus major) (X. Chen et al., 2018). A downstream target of miR-19b-3p is MAPK1, a regulator of the cell cycle that was found to be hypoxia-upregulated at both the mRNA and protein levels (X. Chen et al., 2018). In addition, naked mole-rats shared the same upregulation of miR-184-3p as hypoxia-exposed sea cucumbers (Apostichopus japonicus) where it was proposed to attenuate cellular autophagy (Huo et al., 2017). While the hypoxiadownregulation of miR-1-3p in naked mole-rats contrasts miR-1-3p upregulation in hypoxic sea cucumbers, this opposing directionality is interesting as both the up and downregulation of miR-1 can be beneficial for hypoxic adaptations, depending on the microenvironment present (Huo et al., 2017). 


\subsection{Conclusion}

With research on the uniqueness of naked mole-rat adaptations rapidly growing, our findings highlight the existence of a miRNA-mediated mechanism for facilitating survival as well as hypometabolic regulation in this champion hypoxia-tolerant mammal. Of the 212 conserved miRNAs identified, only 18 were differentially expressed in hypoxic brains where they targeted specific pathways involved in coordinating metabolic fuels during hypoxia with a reliance on anaerobic metabolism and suppressing fructose usage during hypoxia. Other significant miRNA subsets promoted energy-saving during hypometabolism via the suppression of protein translation and cellular proliferation, and activation of neuroprotective signalling processes. Our understanding of miRNA coordination during oxygen-limitation is growing along with our understanding of the molecular adaptations used to facilitate this complex response. 


\subsection{References}

Al-attar, R., Childers, C. L., Nguyen, V. C., Pamenter, M. E., \& Storey, K. B. (2020). Differential protein phosphorylation is responsible for hypoxia-induced regulation of the Akt/mTOR pathway in naked mole-rats. Comparative Biochemistry and Physiology Part A: Molecular \& Integrative Physiology, 242, 110653. https://doi.org/10.1016/J.CBPA.2020.110653

Andersen, C. L., Jensen, J. L., \& Ørntoft, T. F. (2004). Normalization of real-time quantitative reverse transcription-PCR data: A model-based variance estimation approach to identify genes suited for normalization, applied to bladder and colon cancer data sets. Cancer Research, 64(15), 5245-5250.

Aronesty, E. (2011). ea-utils: Command-line tools for processing biological sequencing data. Retrieved from https:/github.com/ExpressionAnalysis/ea-utils

Ashburner, M., Ball, C. A., Blake, J. A., Botstein, D., Butler, H., Cherry, J. M., ... Sherlock, G. (2000). Gene Ontology: tool for the unification of biology. Nature Genetics, 25(1), 25-29.

Bartel, D. (2004). MicroRNAs genomics, biogenesis, mechanism, and function. Cell, 116(2), 281297. Retrieved from http://www.cell.com/article/S0092867404000455/fulltext

Bavelloni, A., Ramazzotti, G., Poli, A., Piazzi, M., Focaccia, E., Blalock, W., \& Faenza, I. (2017). MiRNA-210: A current overview. Anticancer Research, 37(12), 6511-6521. Retrieved from http://www.ncbi.nlm.nih.gov/pubmed/29187425

Bensaad, K., Tsuruta, A., Selak, M. A., Vidal, M. N. C., Nakano, K., Bartrons, R., ... Vousden, K. H. (2006). TIGAR, a p53-inducible regulator of glycolysis and apoptosis. Cell, 126(1), 107-120. https://doi.org/10.1016/j.cell.2006.05.036

Betel, D., Wilson, M., Gabow, A., Marks, D. S., \& Sander, C. (2007). The microRNA.org resource: targets and expression. Nucleic Acids Research, 36(Database), D149-D153.

Biggar, K. K., Wu, C.-W., \& Storey, K. B. (2014). High-throughput amplification of mature microRNAs in uncharacterized animal models using polyadenylated RNA and stem-loop reverse transcription polymerase chain reaction. In Analytical Biochemistry (Vol. 462).

Boutilier, R. G. (2001). Mechanisms of cell survival in hypoxia and hypothermia. Journal of Experimental Biology, 204(18), 3171-3181. Retrieved from https://pubmed.ncbi.nlm.nih.gov/11581331/

Brackmann, F. A., Link, A. S., Jung, S., Richter, M., Zoglauer, D., Walkinshaw, G., ... Trollmann, R. (2013). Activin A regulation under global hypoxia in developing mouse brain. Brain Research, 1531, 65-74. https://doi.org/10.1016/j.brainres.2013.07.039

Bruning, U., Cerone, L., Neufeld, Z., Fitzpatrick, S. F., Cheong, A., Scholz, C. C., ... Taylor, C. T. (2011). MicroRNA-155 promotes resolution of hypoxia-inducible factor 1alpha activity during prolonged hypoxia. Molecular and Cellular Biology, 31(19), 4087-4096. https://doi.org/10.1128/MCB.01276-10 
Bustin, S. A., Benes, V., Garson, J. A., Hellemans, J., Huggett, J., Kubista, M., ... Wittwer, C. T. (2009). The MIQE guidelines: minimum information for publication of quantitative real-time PCR experiments. Clinical Chemistry, 55(4), 611-622.

Chen, D., Hu, S., Wu, Z., Liu, J., \& Li, S. (2018). The role of miR-132 in regulating neural stem cell proliferation, differentiation and neuronal maturation. Cellular Physiology and Biochemistry, 47(6), 2319-2330. https://doi.org/10.1159/000491543

Chen, X., Qu, Y., Cheng, Y., Wang, J., Lei, X., Song, G., ... Lei, F. (2018). MiR-19b-3p regulates MAPK1 expression in embryonic fibroblasts from the Great tit (Parus major) under hypoxic conditions. Cellular Physiology and Biochemistry, 46(2), 546-560. Retrieved from http://www.ncbi.nlm.nih.gov/pubmed/29617681

Chung, D., Dzal, Y. A., Seow, A., Milsom, W. K., \& Pamenter, M. E. (2016). Naked mole-rats exhibit metabolic but not ventilatory plasticity following chronic sustained hypoxia. Proceedings of the Royal Society B: Biological Sciences, 283(1827), 20160216. https://doi.org/10.1098/rspb.2016.0216

Cimmino, F., Avitabile, M., Lasorsa, V. A., Montella, A., Pezone, L., Cantalupo, S., ... Capasso, M. (2019). HIF-1 transcription activity: HIF1A driven response in normoxia and in hypoxia. BMC Medical Genetics, 20(1), 37. https://doi.org/10.1186/s12881-019-0767-1

Clovis, Y. M., Enard, W., Marinaro, F., Huttner, W. B., \& De Pietri Tonelli, D. (2012). Convergent repression of Foxp2 3'UTR by miR-9 and miR-132 in embryonic mouse neocortex: implications for radial migration of neurons. Development, 139(18), 3332-3342. https://doi.org/10.1242/dev.078063

Cui, Y., Chen, L.-G., Yao, H.-B., Zhang, J., \& Ding, K.-F. (2018). Upregulation of microRNA383 inhibits the proliferation, migration and invasion of colon cancer cells. Oncology Letters, 15(1), 1184-1190. https://doi.org/10.3892/ol.2017.7409

D’Ignazio, L., \& Rocha, S. (2016). Hypoxia induced NF-кB. Cells, 5(1). https://doi.org/10.3390/cells5010010

Edrey, Y. H., Hanes, M., Pinto, M., Mele, J., \& Buffenstein, R. (2011). Successful aging and sustained good health in the naked mole-rat: a long-lived mammalian model for biogerontology and biomedical research. ILAR Journal, 52(1), 41-53. https://doi.org/10.1093/ilar.52.1.41

English, S. G., Hadj-Moussa, H., \& Storey, K. B. (2018). MicroRNAs regulate survival in oxygendeprived environments. The Journal of Experimental Biology, 221(23), jeb190579.

Fan, Y., Ding, S., Sun, Y., Zhao, B., Pan, Y., \& Wan, J. (2018). MiR-377 regulates inflammation and angiogenesis in rats after cerebral ischemic injury. Journal of Cellular Biochemistry, 119(1), 327-337. https://doi.org/10.1002/jcb.26181

Fang, Z., He, L., Jia, H., Huang, Q., Chen, D., \& Zhang, Z. (2017). The miR-383-LDHA axis regulates cell proliferation, invasion and glycolysis in hepatocellular cancer. Iranian Journal 
of Basic Medical Sciences, 20(2), 187-192. https://doi.org/10.22038/ijbms.2017.8246

Florio, P., Gazzolo, D., Luisi, S., \& Petraglia, F. (2007). Activin A in brain injury. Advances in Clinical Chemistry, 43, 117-130. https://doi.org/10.1016/s0065-2423(06)43004-3

Friedman, R. C., Farh, K. K.-H., Burge, C. B., \& Bartel, D. P. (2008). Most mammalian mRNAs are conserved targets of microRNAs. Genome Research, 19(1), 92-105.

Gaidatzis, D., van Nimwegen, E., Hausser, J., \& Zavolan, M. (2007). Inference of miRNA targets using evolutionary conservation and pathway analysis. BMC Bioinformatics, 8(1), 69.

Garcia, D. M., Baek, D., Shin, C., Bell, G. W., Grimson, A., \& Bartel, D. P. (2011). Weak seedpairing stability and high target-site abundance decrease the proficiency of lsy- 6 and other microRNAs. Nature Structural \& Molecular Biology, 18(10), 1139-1146.

Ge, L., Cai, Y., Ying, F., Liu, H., Zhang, D., He, Y., ... Xia, Z. (2019). miR-181c-5p exacerbates hypoxia reoxygenation-induced cardiomyocyte apoptosis via targeting PTPN4. Oxidative Medicine and Cellular Longevity, 2019, 1-15. https://doi.org/10.1155/2019/1957920

Gorr, T. A. (2004). Daphnia and Drosophila: two invertebrate models for O2 responsive and HIFmediated regulation of genes and genomes. International Congress Series, 1275, 55-62. https://doi.org/10.1016/J.ICS.2004.08.068

Haase, V. H. (2006). The VHL/HIF oxygen-sensing pathway and its relevance to kidney disease. Kidney International, 69(8), 1302-1307. https://doi.org/10.1038/SJ.KI.5000221

Hadar, A., Milanesi, E., Walczak, M., Puzianowska-Kuźnicka, M., Kuźnicki, J., Squassina, A., ... Gurwitz, D. (2018). SIRT1, miR-132 and miR-212 link human longevity to Alzheimer's Disease. Scientific Reports, 8(1), 8465. https://doi.org/10.1038/s41598-018-26547-6

Hadj-Moussa, H., Logan, S. M., Seibel, B. A., \& Storey, K. B. (2018). Potential role for microRNA in regulating hypoxia-induced metabolic suppression in jumbo squids. Biochimica et Biophysica Acta - Gene Regulatory Mechanisms, 1861(6), 586-593. Retrieved from https://doi.org/10.1016/j.bbagrm.2018.04.007

Hadj-Moussa, H., \& Storey, K. B. (2018). Micromanaging freeze tolerance: the biogenesis and regulation of neuroprotective microRNAs in frozen brains. Cellular and Molecular Life Sciences, 75(19), 3635-3647. Retrieved from https://doi.org/10.1007/s00018-018-2821-0

Hadj-Moussa, H., \& Storey, K. B. (2020). The OxymiR response to oxygen limitation: a comparative microRNA perspective. The Journal of Experimental Biology, 223(Pt 10). https://doi.org/10.1242/jeb.204594

Hadj-Moussa, H., Zhang, J., Pifferi, F., Perret, M., \& Storey, K. B. (2020). Profiling torporresponsive microRNAs in muscles of the hibernating primate Microcebus murinus. Biochimica et Biophysica Acta (BBA) - Gene Regulatory Mechanisms, 1863(1), 194473. https://doi.org/10.1016/j.bbagrm.2019.194473 
Hansen, K. F., Sakamoto, K., Aten, S., Snider, K. H., Loeser, J., Hesse, A. M., .. Obrietan, K. (2016). Targeted deletion of miR-132/-212 impairs memory and alters the hippocampal transcriptome. Learning \& Memory (Cold Spring Harbor, N.Y.), 23(2), 61-71. https://doi.org/10.1101/lm.039578.115

Haviv, R., Oz, E., \& Soreq, H. (2018). The stress-responding miR-132-3p shows evolutionarily conserved pathway interactions. Cellular and Molecular Neurobiology, 38(1), 141-153. https://doi.org/10.1007/s10571-017-0515-z

Hawkins, L. J., Hadj-Moussa, H., Nguyen, V. C., Pamenter, M. E., \& Storey, K. B. (2019). Naked mole-rats activate neuroprotective proteins during hypoxia. Journal of Experimental Zoology Part A: Ecological and Integrative Physiology, 331(10), 571-576. https://doi.org/10.1002/jez.2321

Hayden, M. S., \& Ghosh, S. (2012). NF- B, the first quarter-century: remarkable progress and outstanding questions. Genes \& Development, 26(3), 203-234. Retrieved from http://www.ncbi.nlm.nih.gov/pubmed/22302935

Henry, R. J., Doran, S. J., Barrett, J. P., Meadows, V. E., Sabirzhanov, B., Stoica, B. A., ... Faden, A. I. (2019). Inhibition of miR-155 limits neuroinflammation and improves functional recovery after experimental traumatic brain injury in mice. Neurotherapeutics, 16(1), 216230. https://doi.org/10.1007/s13311-018-0665-9

Hewitson, J. P., Shah, K. M., Brown, N., Grevitt, P., Hain, S., Newling, K., ... Lagos, D. (2019). miR-132 suppresses transcription of ribosomal proteins to promote protective Th1 immunity. EMBO Reports, 20(4). https://doi.org/10.15252/embr.201846620

Hochachka, P. W., Buck, L. T., Doll, C. J., \& Land, S. C. (1996). Unifying theory of hypoxia tolerance: molecular/metabolic defense and rescue mechanisms for surviving oxygen lack. Proceedings of the National Academy of Sciences, 93(18), 9493-9498. https://doi.org/10.1073/PNAS.93.18.9493

Holtze, S., Braude, S., Lemma, A., Koch, R., Morhart, M., Szafranski, K., ... Hildebrandt, T. B. (2018). The microenvironment of naked mole-rat burrows in East Africa. African Journal of Ecology, 56(2), 279-289. https://doi.org/10.1111/aje.12448

Houlahan, C. R., Kirby, A. M., Dzal, Y. A., Fairman, G. D., \& Pamenter, M. E. (2018). Divergent behavioural responses to acute hypoxia between individuals and groups of naked mole-rats. Comparative Biochemistry and Physiology Part B: Biochemistry and Molecular Biology, $224,38-44$.

Hoyeck, M. P., Hadj-Moussa, H., \& Storey, K. B. (2019). Estivation-responsive microRNAs in a hypometabolic terrestrial snail. PeerJ, 7, e6515. Retrieved from https://peerj.com/articles/6515

Huo, D., Sun, L., Li, X., Ru, X., Liu, S., Zhang, L., ... Yang, H. (2017). Differential expression of miRNAs in the respiratory tree of the sea cucumber Apostichopus japonicus under hypoxia stress. G3 (Bethesda, Md.), 7(11), 3681-3692. Retrieved from 
http://www.ncbi.nlm.nih.gov/pubmed/28916650

Jiang, J., Xie, C., Liu, Y., Shi, Q., \& Chen, Y. (2019). Up-regulation of miR-383-5p suppresses proliferation and enhances chemosensitivity in ovarian cancer cells by targeting TRIM27. Biomedicine \& Pharmacotherapy, 109, 595-601. https://doi.org/10.1016/J.BIOPHA.2018.10.148

Jiang, S., Zhang, H. W., Lu, M. H., He, X. H., Li, Y., Gu, H., .. Wang, E. D. (2010). MicroRNA155 functions as an oncomiR in breast cancer by targeting the suppressor of cytokine signaling 1 gene. Cancer Research, 70(8), 3119-3127. https://doi.org/10.1158/00085472.CAN-09-4250

Kanehisa, M., \& Goto, S. (2000). KEGG: kyoto encyclopedia of genes and genomes. Nucleic Acids Research, 28(1), 27-30.

Kertesz, M., Iovino, N., Unnerstall, U., Gaul, U., \& Segal, E. (2007). The role of site accessibility in microRNA target recognition. Nature Genetics, 39(10), 1278-1284.

Kim, E. B., Fang, X., Fushan, A. A., Huang, Z., Lobanov, A. V., Han, L., ... Gladyshev, V. N. (2011). Genome sequencing reveals insights into physiology and longevity of the naked molerat. Nature, 479(7372), 223-227. https://doi.org/10.1038/nature10533

Krek, A., Grün, D., Poy, M. N., Wolf, R., Rosenberg, L., Epstein, E. J., ... Rajewsky, N. (2005). Combinatorial microRNA target predictions. Nature Genetics, 37(5), 495-500.

Slemc, L., \& Kunej, T. (2016). Transcription factor HIF1A: Downstream targets associated pathways, polymorphic Hypoxia Response Element (HRE) sites, and initiative for standardization of reporting in scientific literature. Tumour Biology: The Journal of the International Society for Oncodevelopmental Biology and Medicine, 37(11). https://doi.org/10.1007/S13277-016-5331-4

Lagos, D., Pollara, G., Henderson, S., Gratrix, F., Fabani, M., Milne, R. S. B., ... Boshoff, C. (2010). miR-132 regulates antiviral innate immunity through suppression of the p300 transcriptional co-activator. Nature Cell Biology, 12(5), 513-519. https://doi.org/10.1038/ncb2054

Larson, J., \& Park, T. J. (2009). Extreme hypoxia tolerance of naked mole-rat brain. NeuroReport, 20(18), 1634-1637. https://doi.org/10.1097/WNR.0b013e32833370cf

Law, C. W., Chen, Y., Shi, W., \& Smyth, G. K. (2014). Voom: precision weights unlock linear model analysis tools for RNA-seq read counts. Genome Biology, 15(2), R29.

Lenz, M., Müller, F.-J., Zenke, M., \& Schuppert, A. (2016). Principal components analysis and the reported low intrinsic dimensionality of gene expression microarray data. Scientific Reports, 6(1), 25696. Retrieved from http://www.nature.com/articles/srep25696

Lewis, B. P., Burge, C. B., \& Bartel, D. P. (2005). Conserved seed pairing, often flanked by adenosines, indicates that thousands of human $\mathrm{h}$ genes are microRNA targets. Cell, 120(1), 
$15-20$.

Li, H., Handsaker, B., Wysoker, A., Fennell, T., Ruan, J., Homer, N., ... 1000 Genome Project Data Processing Subgroup. (2009). The sequence alignment/map format and SAMtools. Bioinformatics, 25(16), 2078-2079.

Liu, F. J., Kaur, P., Karolina, D. S., Sepramaniam, S., Armugam, A., Wong, P. T. H., \& Jeyaseelan, K. (2015). MiR-335 regulates Hif-1 $\alpha$ to reduce cell death in both mouse cell line and rat $\begin{array}{lllll}\text { ischemic } \quad \text { models. } & \text { PLOSE, } & \text { 10(6), } 0128432 .\end{array}$ https://doi.org/10.1371/journal.pone.0128432

Liu, N., Xia, W.-Y., Liu, S.-S., Chen, H.-Y., Sun, L., Liu, M.-Y., ... Gao, J.-X. (2016). MicroRNA101 targets von Hippel-Lindau tumor suppressor (VHL) to induce HIF1 $\alpha$ mediated apoptosis and cell cycle arrest in normoxia condition. Scientific Reports, 6(1), 20489. https://doi.org/10.1038/srep20489

Logan, S. M., Szereszewski, K. E., Bennet, N. C., Hart, D. W., van Jaarsveld, B., Pamenter, M. E., \& Storey, K. B. (2020). The brains of six African mole-rat species show divergent responses to hypoxia. Journal of Experimental Biology. https://doi.org/10.1242/jeb.215905

Luo, W., \& Brouwer, C. (2013). Pathview: an R/Bioconductor package for pathway-based data integration and visualization. Bioinformatics, 29(14), 1830-1831.

Maistrovski, Y., Biggar, K., \& Storey, K. (2012). HIF-1 $\alpha$ regulation in mammalian hibernators: Role of non-coding RNA in HIF-1 $\alpha$ control during torpor in ground squirrels and bats. Journal of Comparative Physiology. B, Biochemical, Systemic, and Environmental Physiology, 182(6). https://doi.org/10.1007/S00360-012-0662-Y

Masoud, G. N., \& Li, W. (2015). HIF-1 $\alpha$ pathway: role, regulation and intervention for cancer therapy. Acta Pharmaceutica Sinica. $3, \quad$ B https://doi.org/10.1016/J.APSB.2015.05.007

Mehta, A., \& Baltimore, D. (2016). MicroRNAs as regulatory elements in immune system logic. Nature Reviews Immunology, 16(5), 279-294. https://doi.org/10.1038/nri.2016.40

Michael, G., \& Philip, W. (1999). Metabolic depression in animals: Physiological perspectives and biochemical generalizations. Biological Reviews of the Cambridge Philosophical Society, 74(1). https://doi.org/10.1017/S0006323198005258

Mo, J.-L., Liu, Q., Kou, Z.-W., Wu, K.-W., Yang, P., Chen, X.-H., \& Sun, F.-Y. (2018). MicroRNA-365 modulates astrocyte conversion into neuron in adult rat brain after stroke by targeting Pax6. Glia, 66(7), 1346-1362. https://doi.org/10.1002/glia.23308

Morin, P., McMullen, D. C., \& Storey, K. B. (2005). HIF-1 $\alpha$ involvement in low temperature and anoxia survival by a freeze tolerant insect. Molecular and Cellular Biochemistry, 280(1-2), 99-106. https://doi.org/10.1007/s11010-005-8236-x

Mukerji, S. S., Katsman, E. A., Wilber, C., Haner, N. A., Selman, W. R., \& Hall, A. K. (2007). 
Activin is a neuronal survival factor that is rapidly increased after transient cerebral ischemia and hypoxia in mice. Journal of Cerebral Blood Flow \& Metabolism, 27(6), 1161-1172. https://doi.org/10.1038/sj.jcbfm.9600423

Nguyen, V., Deck, C., \& Pamenter, M. (2019). Naked mole-rats reduce the expression of ATPdependent but not ATP-independent heat shock proteins in acute hypoxia. The Journal of Experimental Biology, 222(Pt 22). https://doi.org/10.1242/JEB.211243

O’Brien, J., Hayder, H., Zayed, Y., \& Peng, C. (2018). Overview of microRNA biogenesis, mechanisms of actions, and circulation. Frontiers in Endocrinology, 9, 402. https://doi.org/10.3389/fendo.2018.00402

Oppelt, S. A., Zhang, W., \& Tolan, D. R. (2017). Specific regions of the brain are capable of fructose metabolism. Brain Research, 1657, 312-322. https://doi.org/10.1016/j.brainres.2016.12.022

Pamenter, M. E., Dzal, Y. A., Thompson, W. A., \& Milsom, W. K. (2019). Do naked mole-rats accumulate a metabolic acidosis or an oxygen debt in severe hypoxia? The Journal of Experimental Biology, 222(Pt 3). https://doi.org/10.1242/jeb.191197

Pamenter, M. E., Lau, G. Y., Richards, J. G., \& Milsom, W. K. (2018). Naked mole-rat brain mitochondria electron transport system flux and $\mathrm{H}+$ leak are reduced during acute hypoxia. Journal of Experimental Biology, 221(4). https://doi.org/10.1242/JEB.171397

Paraskevopoulou, M. D., Georgakilas, G., Kostoulas, N., Vlachos, I. S., Vergoulis, T., Reczko, M., ... Hatzigeorgiou, A. G. (2013). DIANA-microT web server v5.0: service integration into miRNA functional analysis workflows. Nucleic Acids Research, 41(Web Server issue), W169-73. https://doi.org/10.1093/nar/gkt393

Park, T. J., Reznick, J., Peterson, B. L., Blass, G., Omerbašić, D., Bennett, N. C., ... Lewin, G. R. (2017). Fructose-driven glycolysis supports anoxia resistance in the naked mole-rat. Science (New York, N.Y.), 356(6335), 307-311. https://doi.org/10.1126/science.aab3896

Peltier, H. J., \& Latham, G. J. (2008). Normalization of microRNA expression levels in quantitative RT-PCR assays: Identification of suitable reference RNA targets in normal and cancerous human solid tissues. Rna, 14(5), 844-852.

Quinlan, S., \& Jimenez-Mateos, E. (2016). Can we protect the brain via preconditioning? Role of microRNAs in neuroprotection. Neural Regeneration Research, 11(3), 388. https://doi.org/10.4103/1673-5374.179037

R Core Team. (2017). R: A language and environment for statistical computing. Retrieved from https://www.r-project.org/

Remenyi, J., van den Bosch, M. W. M., Palygin, O., Mistry, R. B., McKenzie, C., Macdonald, A., ... Pankratov, Y. (2013). miR-132/212 knockout mice reveal roles for these miRNAs in regulating cortical synaptic transmission and plasticity. PLoS ONE, 8(4), e62509. https://doi.org/10.1371/journal.pone.0062509 
Riggs, C. L., Summers, A., Warren, D. E., Nilsson, G. E., Lefevre, S., Dowd, W. W., ... Podrabsky, J. E. (2018). Small non-coding RNA expression and vertebrate anoxia tolerance. Frontiers in Genetics, 9, 230. Retrieved from http://www.ncbi.nlm.nih.gov/pubmed/30042786

Ritchie, M. E., Phipson, B., Wu, D., Hu, Y., Law, C. W., Shi, W., \& Smyth, G. K. (2015). Limma powers differential expression analyses for RNA-sequencing and microarray studies. Nucleic Acids Research, 43(7), e47-e47.

Rodriguez, K. A., Osmulski, P. A., Pierce, A., Weintraub, S. T., Gaczynska, M., \& Buffenstein, R. (2014). A cytosolic protein factor from the naked mole-rat activates proteasomes of other species and protects these from inhibition. Biochimica et Biophysica Acta (BBA) - Molecular Basis of Disease, 1842(11), 2060-2072. https://doi.org/10.1016/J.BBADIS.2014.07.005

Rogatzki, M. J., Ferguson, B. S., Goodwin, M. L., \& Gladden, L. B. (2015). Lactate is always the end product of glycolysis. Frontiers in Neuroscience, 9, 22. https://doi.org/10.3389/fnins.2015.00022

Schmittgen, T. D., \& Livak, K. J. (2008). Analyzing real-time PCR data by the comparative CT method. Nature Protocols, 3(6), 1101-1108.

Smith, E. S. J., Schuhmacher, L.-N., \& Husson, Z. (2015). The naked mole-rat as an animal model in biomedical research: current perspectives. Open Access Animal Physiology, 7, 137. https://doi.org/10.2147/OAAP.S50376

Sud, N., Zhang, H., Pan, K., Cheng, X., Cui, J., \& Su, Q. (2017). Aberrant expression of microRNA induced by high-fructose diet: implications in the pathogenesis of hyperlipidemia and hepatic insulin resistance. The Journal of Nutritional Biochemistry, 43, 125-131. https://doi.org/10.1016/j.jnutbio.2017.02.003

The Gene Ontology Consortium. (2017). Expansion of the Gene Ontology knowledgebase and resources. Nucleic Acids Research, 45(D1), D331-D338.

Thome, A. D., Harms, A. S., Volpicelli-Daley, L. A., \& Standaert, D. G. (2016). microRNA-155 regulates alpha-synuclein-induced inflammatory responses in models of Parkinson disease. The Journal of Neuroscience, 36(8), 2383-2390. https://doi.org/10.1523/JNEUROSCI.390015.2016

Truettner, J. S., Katyshev, V., Esen-Bilgin, N., Dietrich, W. D., \& Dore-Duffy, P. (2013). Hypoxia alters microRNA expression in rat cortical pericytes. MicroRNA, 2(1), 32-45. https://doi.org/10.2174/2211536611302010005

Wang, D., Ren, J., Ren, H., Fu, J., \& Yu, D. (2018). MicroRNA-132 suppresses cell proliferation in human breast cancer by directly targeting FOXA1. Acta Pharmacologica Sinica, 39(1), 124-131. https://doi.org/10.1038/aps.2017.89

Wang, X. (2008). miRDB: A microRNA target prediction and functional annotation database with a wiki interface. $R N A, 14(6), 1012-1017$. 
Wang, Xinyu, Ren, Y., Wang, Z., Xiong, X., Han, S., Pan, W., .. Yang, M. (2015). Downregulation of 5S rRNA by miR-150 and miR-383 enhances c-Myc-rpL11 interaction and inhibits proliferation of esophageal squamous carcinoma cells. FEBS Letters, 589(24PartB), 3989-3997. https://doi.org/10.1016/j.febslet.2015.11.012

Warnes, G., Bolker, B., Bonebakker, L., Gentleman, R., Liaw, W., Lumley, T., ... Al., E. (2015). gplots: various $R$ programming tools for plotting data. $R$ package.

Xiao, B., Wang, S., Yang, G., Sun, X., Zhao, S., Lin, L., ... Cui, S. (2017). HIF-1a contributes to hypoxia adaptation of the naked mole-rat. Oncotarget, 8(66), 109941-109951. https://doi.org/10.18632/oncotarget.22767

Xu, Zheng, Xiao, S.-B., Xu, P., Xie, Q., Cao, L., Wang, D., ... Fang, L.-R. (2011). miR-365, a novel negative regulator of interleukin-6 gene expression, is cooperatively regulated by Sp1 and NF-kappaB. The Journal of Biological Chemistry, 286(24), 21401-21412. https://doi.org/10.1074/jbc.M110.198630

Xu, Zhou, Zeng, X., Tian, D., Xu, H., Cai, Q., Wang, J., \& Chen, Q. (2014). MicroRNA-383 inhibits anchorage-independent growth and induces cell cycle arrest of glioma cells by targeting CCND1. Biochemical and Biophysical Research Communications, 453(4), 833838. https://doi.org/10.1016/j.bbrc.2014.10.047

Yan, F., Tu, Z., Duan, L., Wang, D., \& Lin, F. (2018). MicroRNA-383 suppresses cell proliferation and invasion in colorectal cancer by directly targeting paired box 6. Molecular Medicine Reports, 17(5), 6893-6901. https://doi.org/10.3892/mmr.2018.8682

Yan, Y., Shi, Y., Wang, C., Guo, P., Wang, J., Zhang, C.-Y., \& Zhang, C. (2015). Influence of a high-altitude hypoxic environment on human plasma microRNA profiles. Scientific Reports, 5(1), 15156. Retrieved from http://www.nature.com/articles/srep15156

Yang, D., Wang, J., Xiao, M., Zhou, T., \& Shi, X. (2016). Role of Mir-155 in controlling HIF-1 $\alpha$ level and promoting endothelial cell maturation. Scientific Reports, 6(1), 35316. https://doi.org/10.1038/srep35316

Yao, C., Shi, X., Zhang, Z., Zhou, S., Qian, T., Wang, Y., ... Yu, B. (2016). Hypoxia-induced upregulation of miR-132 promotes schwann cell migration after sciatic nerve injury by targeting PRKAG3. Molecular Neurobiology, 53(8), 5129-5139. https://doi.org/10.1007/s12035-015-9449-y

Yogarajah, M., Matarin, M., Vollmar, C., Thompson, P. J., Duncan, J. S., Symms, M., ... Sisodiya, S. M. (2016). PAX6, brain structure and function in human adults: advanced MRI in aniridia. Annals of Clinical and Translational Neurology, 3(5), 314-330. https://doi.org/10.1002/acn3.297

Zhang, B., Qiangba, Y., Shang, P., Wang, Z., Ma, J., Wang, L., \& Zhang, H. (2015). A comprehensive microRNA expression profile related to hypoxia adaptation in the Tibetan pig. PLOS ONE, 10(11), e0143260. Retrieved from https://dx.plos.org/10.1371/journal.pone.0143260 
Zhang, J., Hadj-Moussa, H., \& Storey, K. B. (2016). Current progress of high-throughput microRNA differential expression analysis and random forest gene selection for model and non-model systems: an R implementation. Journal of Integrative Bioinformatics, 13(5), 306. Retrieved from http://www.ncbi.nlm.nih.gov/pubmed/28187420

Zhang, J., \& Storey, K. B. (2016). RBioplot: an easy-to-use R pipeline for automated statistical analysis and data visualization in molecular biology and biochemistry. PeerJ, 4, e2436.

Zhang, J., \& Storey, K. B. (2018). RBiomirGS: an all-in-one miRNA gene set analysis solution featuring target mRNA mapping and expression profile integration. PeerJ, 6, e4262.

Zhang, L., Dong, L.-Y., Li, Y.-J., Hong, Z., \& Wei, W.-S. (2012). The microRNA miR-181c controls microglia-mediated neuronal apoptosis by suppressing tumor necrosis factor. Journal of Neuroinflammation, 9(1), 716. https://doi.org/10.1186/1742-2094-9-211

Zhou, W., Yao, Y., Li, J., Wu, D., Zhao, M., Yan, Z., ... Kong, L. (2019). TIGAR attenuates high glucose-induced neuronal apoptosis via an autophagy pathway. Frontiers in Molecular Neuroscience, 12 , 193. https://doi.org/10.3389/fnmol.2019.00193 


\subsection{Figures}

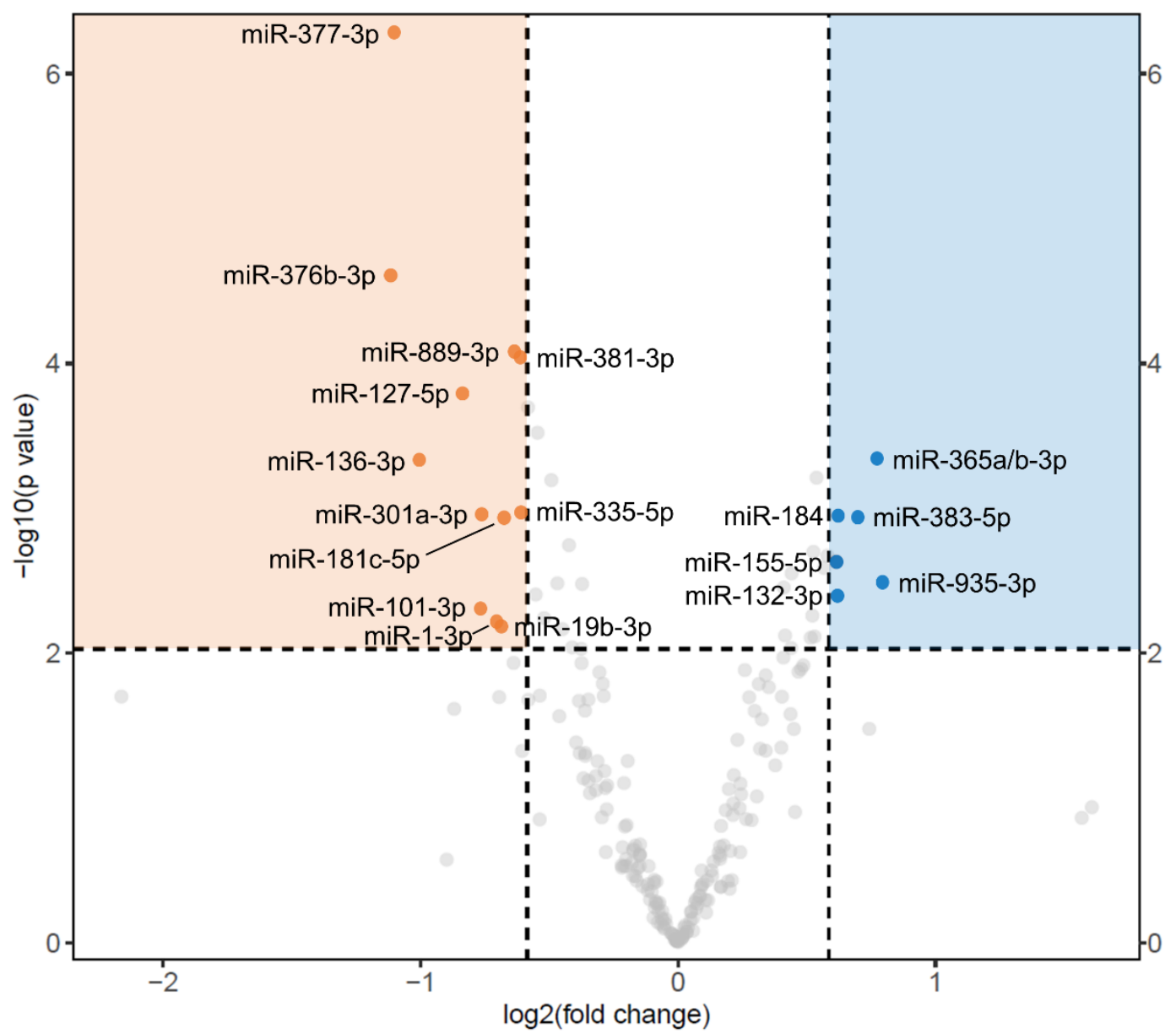

Figure 4.1. Differential expression analysis. Volcano plot for DE results, with $p$-value and fold changes. $\log 2$ transformed fold change and $\log 10 p$-values are plotted. Blue represents statistically significant upregulation greater than 1.5-fold. Orange represents statistically significant downregulations greater than 1.5-fold. Top 18 (based on FDR-corrected $p$ values $<0.05$ ) differentially expressed miRNAs are marked. 


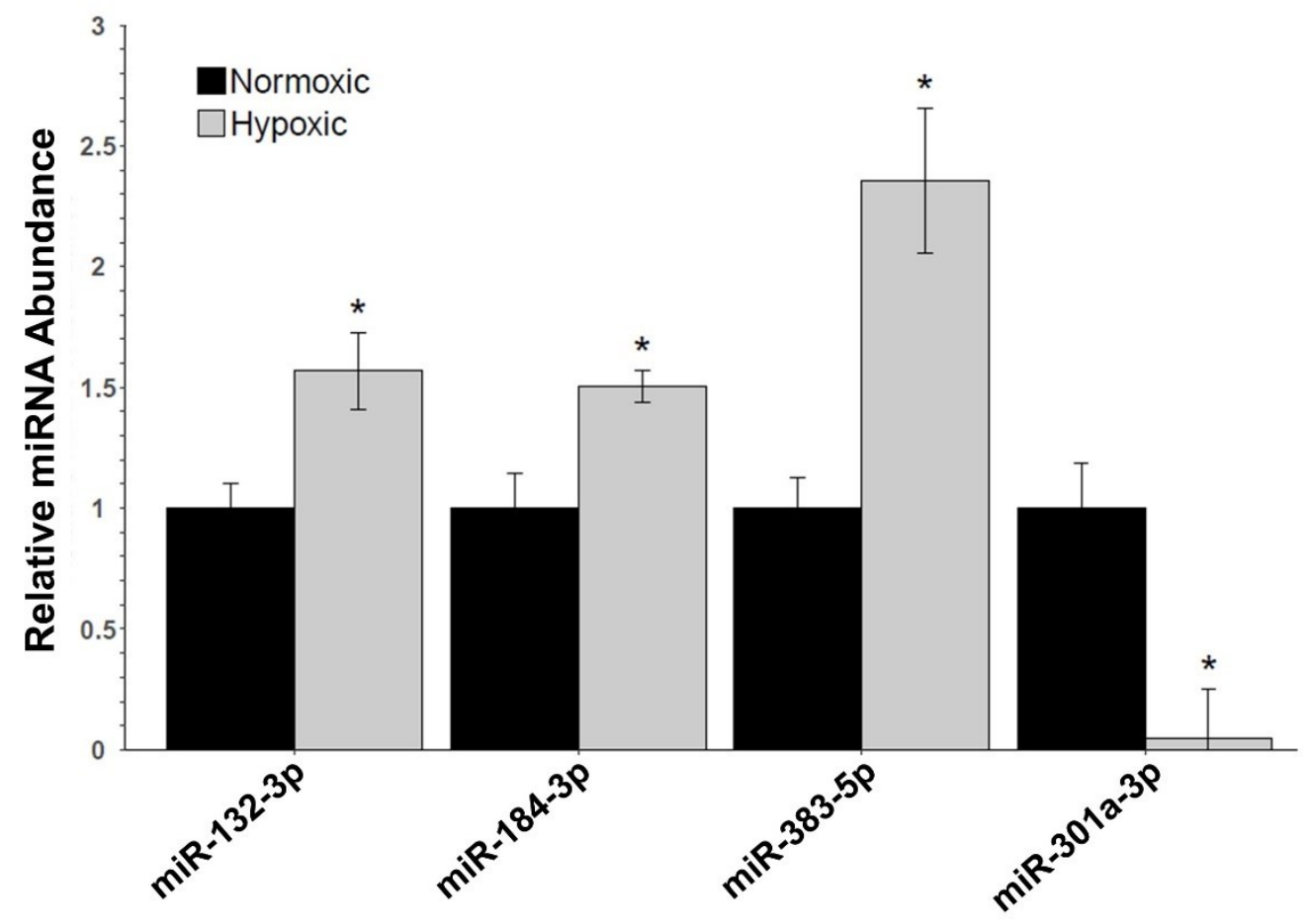

Figure 4.2. RT-qPCR validation of a representative set of significantly differentially expressed miRNAs. Four miRNAs identified to be significantly expressed in miRNA-Seq analysis (miR-132-3p, miR-184-3p, miR-383-5p, and miR-301a-3p) were validate with RT-qPCR. Data were analyzed as means of $n=3-4 \pm$ SEM using a Student's t-test, $* p<$ 0.05 . 
A

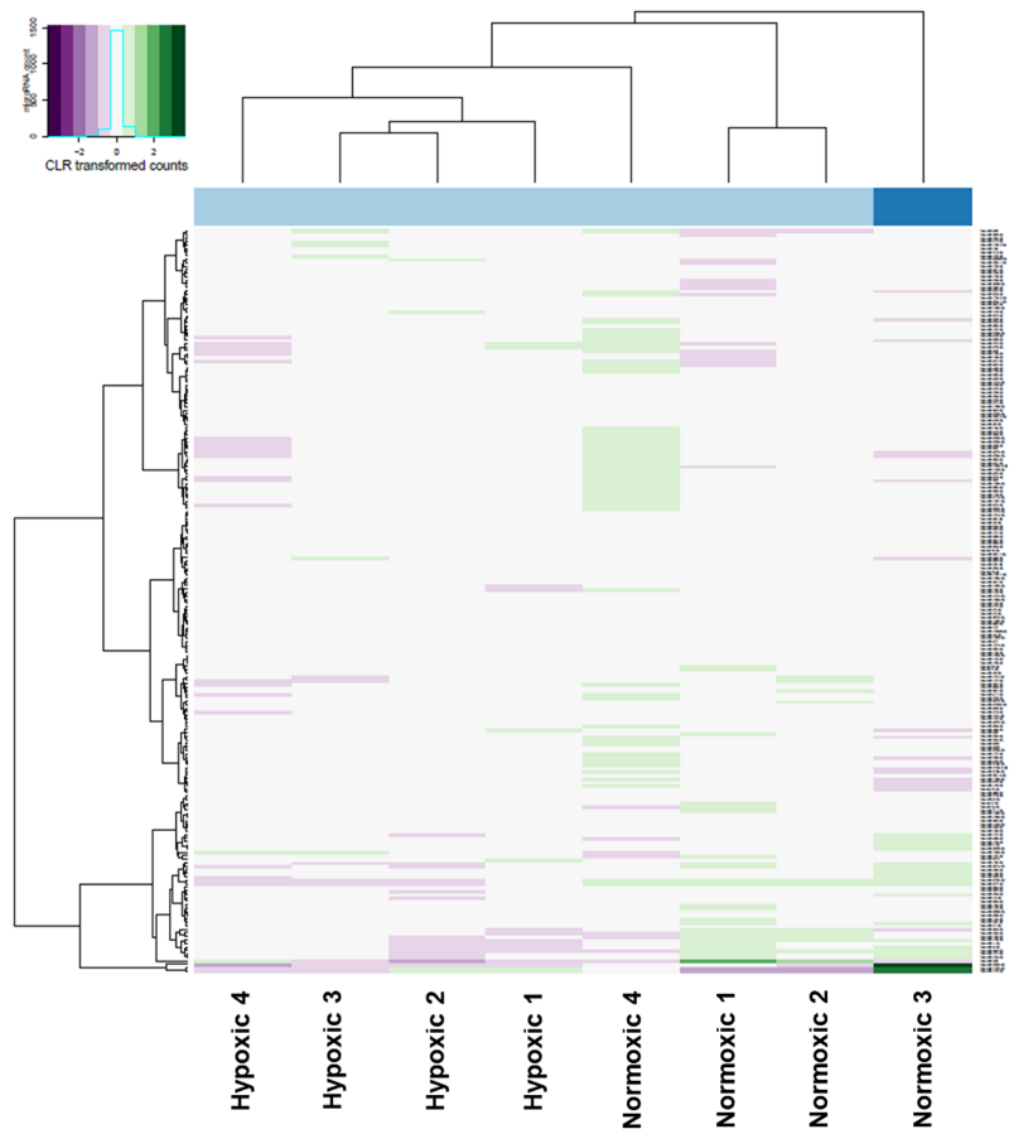

B

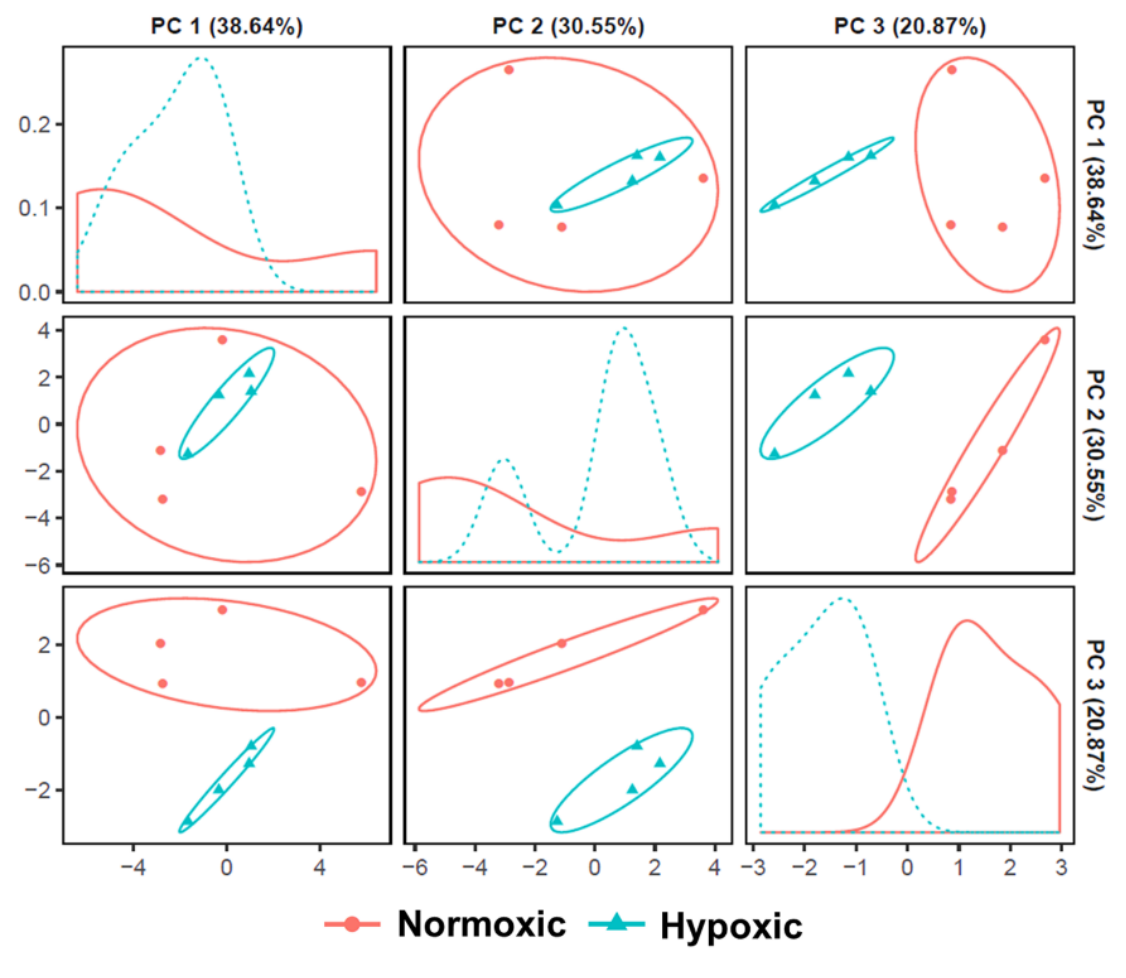

Figure 4.3. Unsupervised clustering analysis of miRNA expression levels. (A) Heatmap for hierarchical clustering, dendrograms for miRNAs and samples. Light and dark blue bars represent the two clusters that samples were divided into based on their global miRNA expression patterns. (B) Principal component analysis (PCA) on PC1, PC2, and PC3, with density plots on the diagonal. 

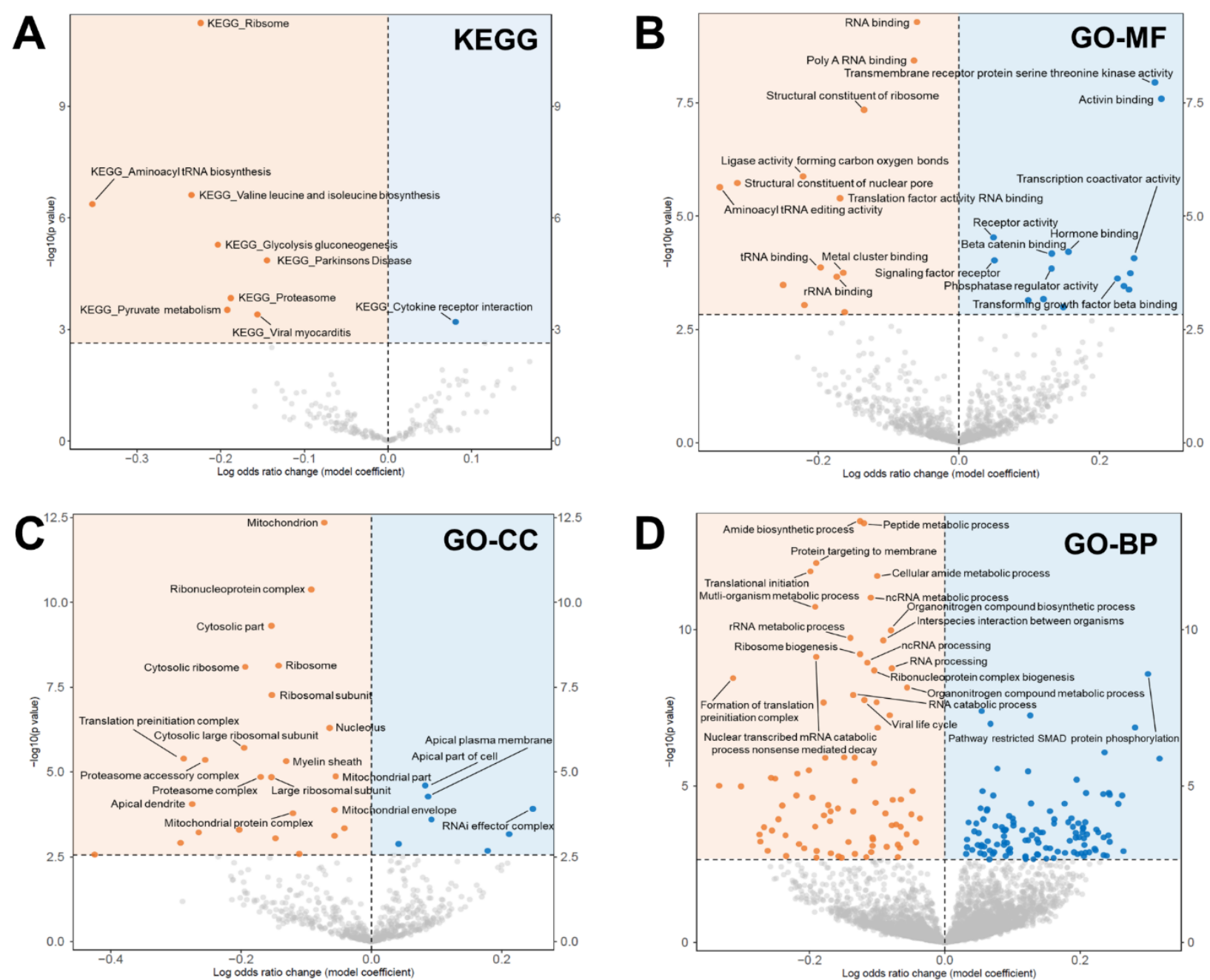

Figure 4.4. Volcano plot for logistic regression based KEGG and GO term gene set enrichment. Blue dots represent significantly enriched gene sets that lack miRNA inhibition during hypoxia based on the miRNA expression profiles. Orange dots represent significantly enriched gene sets that are miRNA-inhibited during hypoxia. Based on FDR corrected $p$-values $<0.05$ and fold change $>1.5$. (A) KEGG Pathway enrichment, (B) GO Term Molecular Function enrichment, (C) GO Term Cellular Component enrichment, and (D) GO Term Biological Process enrichment. The complete results for KEGG and GO GS analysis can be viewed in (Supp. Table S4-S7 found online). 
A

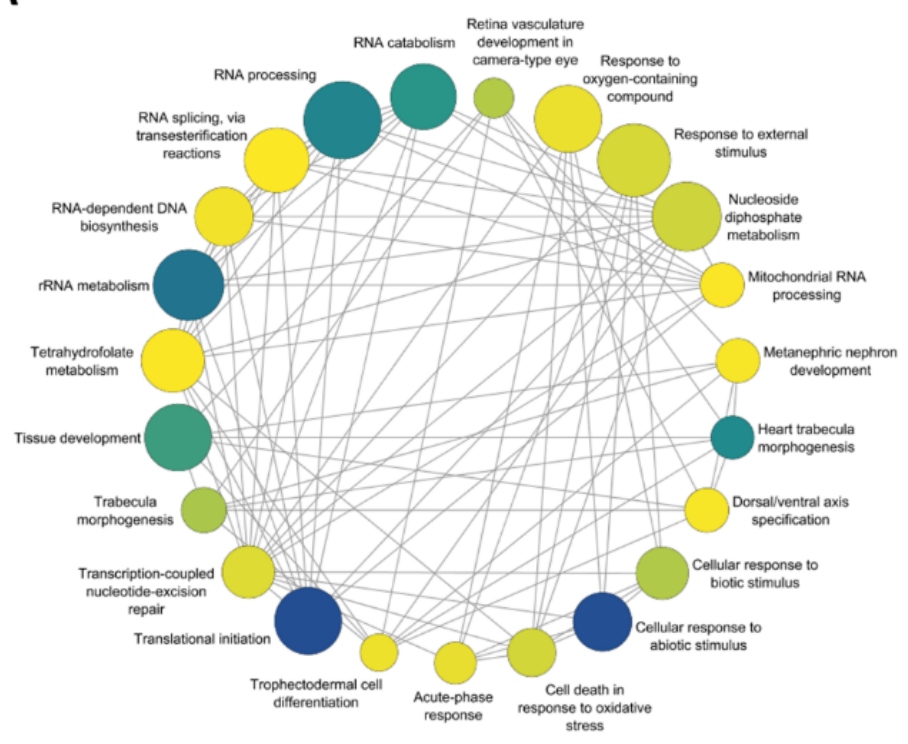

C

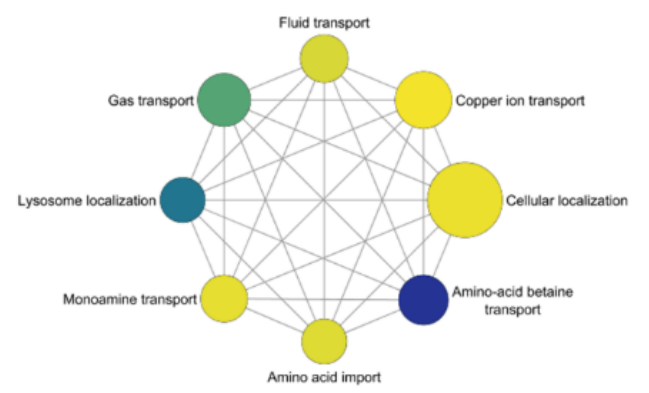

D
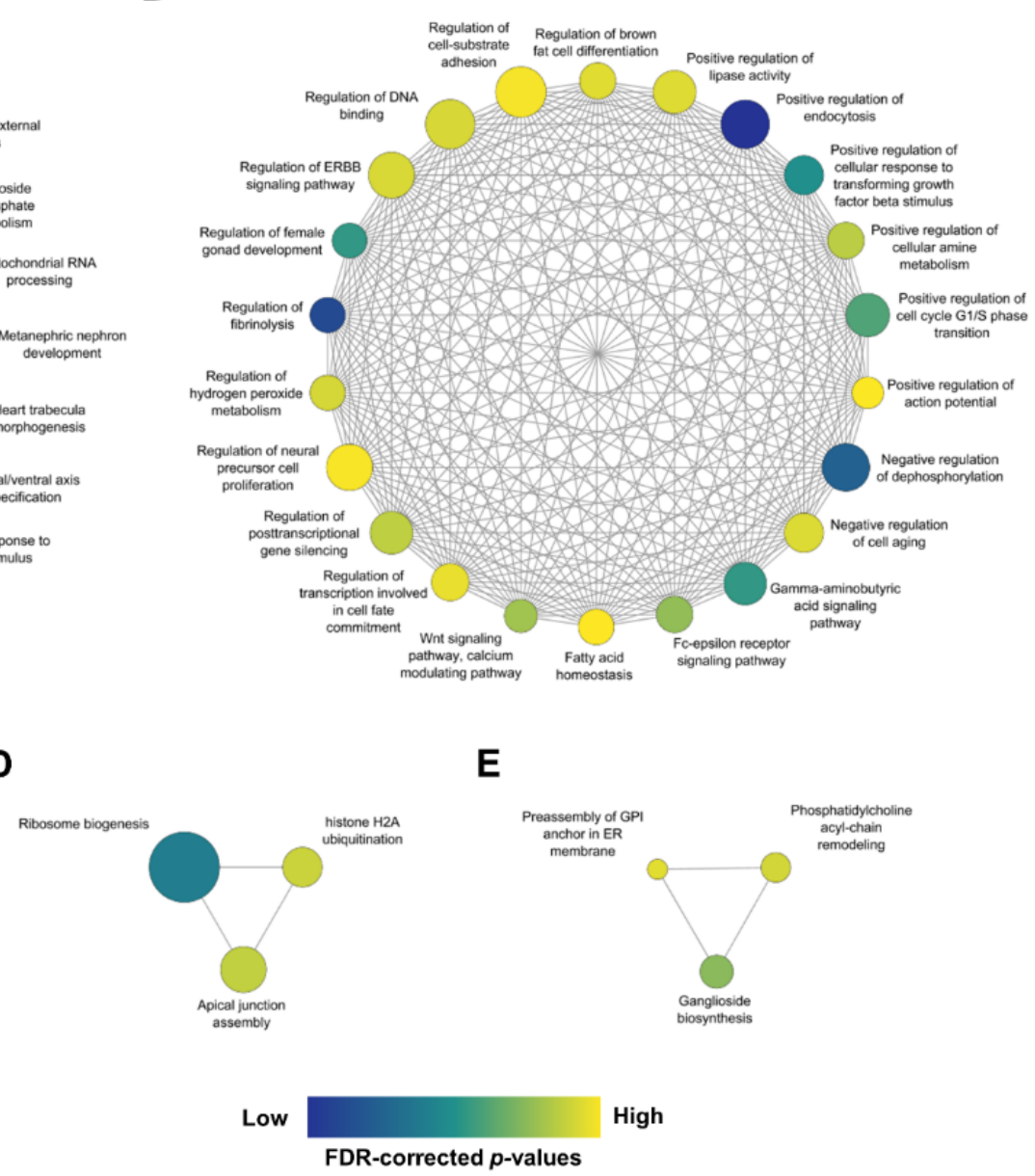

Figure 4.5. Enriched GO Biological Process semantic network in hypoxic naked mole-rat brains. The 200 significantly enriched GO Biological Processes were summarized by REVIGO and the resulting sematic relation network of summary terms was visualized using Cytoscape. (A) The first network was related to RNA metabolism, external responses to stress stimuli, and tissue development. (B) The second largest network was related to signal transduction and transcription factor pathways, neuronal processes, and regulation of post-transcriptional silencing and post-translational modification contained the most sematic relationships. (C) The third network was related to small ion and amino acid transport and localization. (D) The fourth network comprised of only 3 members was related to ribosomal biogenesis and histone ubiquitination, (E) and the fifth network, also with 3 members was related to lipid biosynthesis and remodelling. Node colour represents FDR corrected $p$-values, node size is proportional to the number of genes each term contains, and grey edges represent sematic relationships. The complete results for GO Biological Process analysis can be viewed in (Supp. Table S7 found online). 
A

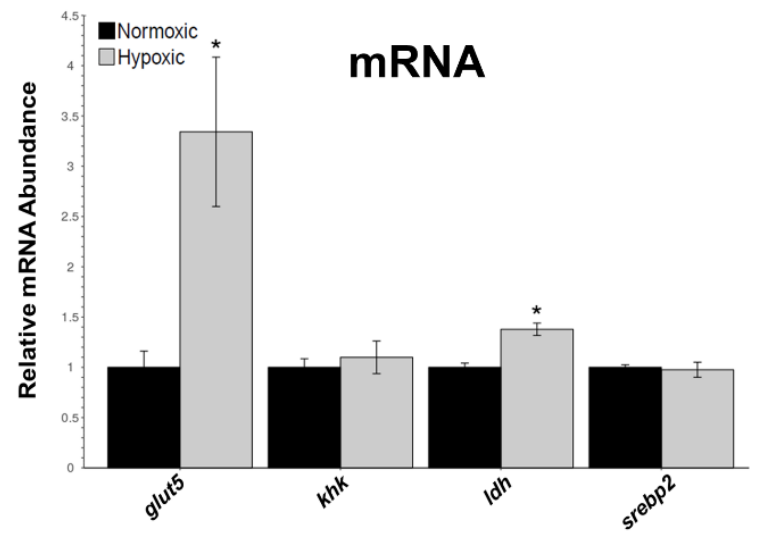

B

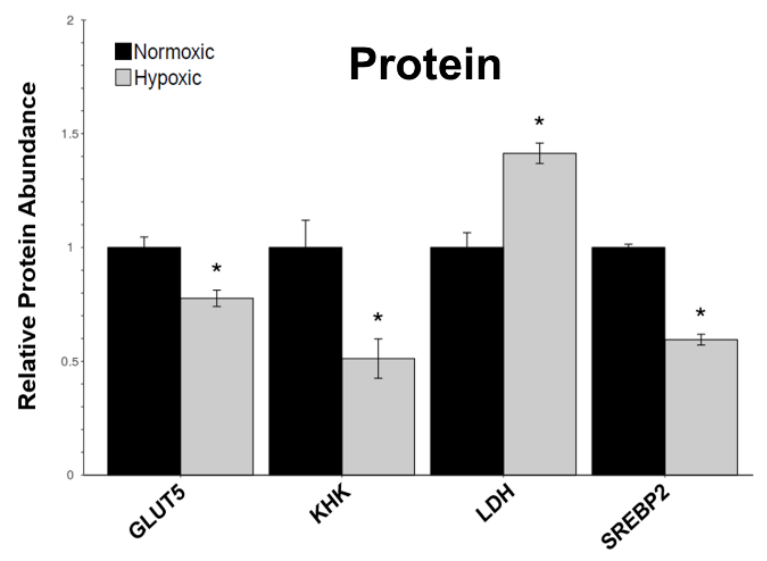

C

Target gene Hypoxia-induced changes in miRNA expression

KHK $\longmapsto \uparrow m i R-365$

LDH $\longmapsto \quad \uparrow m i R-383 \uparrow m i R-935$

SREBP2 $\longmapsto \downarrow m i R-101 a-3 p \downarrow m i R-19 b-3 p$

GLUT5 $\longmapsto$ Not targeted by differentially expressed miRNAs

Figure 4.6. Transcript mRNA and protein levels of miRNA-downstream metabolic glycolytic targets. (A) Relative mRNA abundance levels of glut $5, k h k$, ldh, and srebp2. Data were analyzed as means of $n=3-4 \pm$ SEM using a Student's t-test, ${ }^{*} p<0.05$, relative to normalized normoxic values. (B) Protein levels of GLUT5, KHK, LDH, and SREBP2. Data were analyzed as means of $n=3-4 \pm$ SEM using a Student's t-test, ${ }^{*} p<0.05$, relative to normalized normoxic values. (C) Schematic of hypoxic miRNA targeting on KHK, LDH, SREBP2, and GLUT5. 


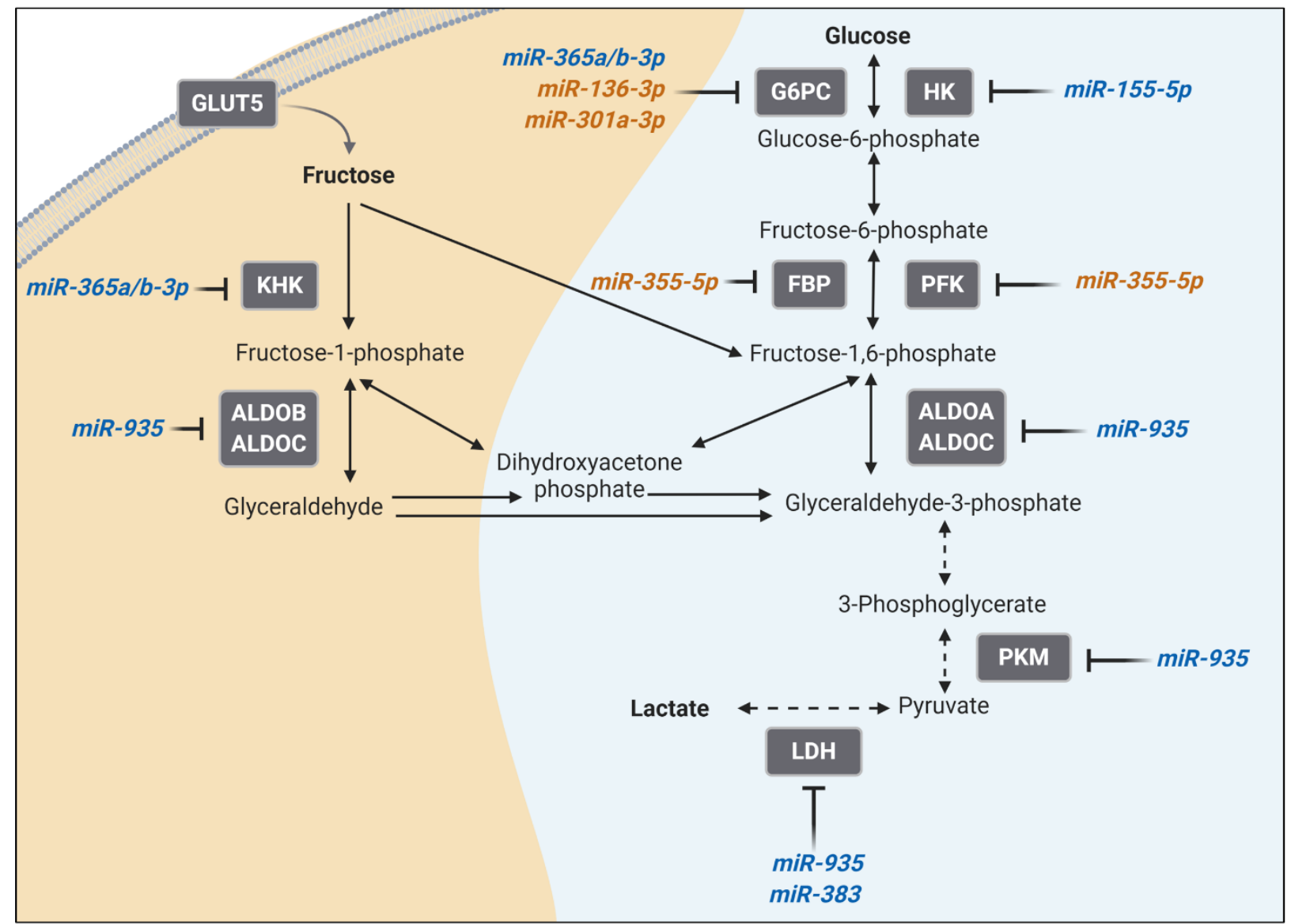

Figure 4.7. Schematic of miRNA-mediated targeting of fructose and glucose anaerobic glycolysis pathways. Blue miRNAs are upregulated during hypoxia while orange miRNAs are downregulated in hypoxic naked mole-rat brains. 


\subsection{Supplementary Materials}

The data supporting this article is present in the figures and has been uploaded in full to Supplementary Table S1-S7. Sequence reads were submitted to the SRA database and can be accessed using the following accession numbers (SRR12668869-SRR12668876). Electronic supplementary material can be found here:

Hadj-Moussa H, Pamenter ME, \& Storey KB. (2021). Hypoxic naked mole-rat brains use microRNA to coordinate hypometabolic fuels and neuroprotective defenses. Journal of Cellular Physiology, 236(7), 5080-5097.

Supplementary Table S1. Primers used for naked mole-rat miRNA and mRNA RT-qPCR analyses.

Supplementary Table S2. Differential miRNA expression table.

Supplementary Table S3. Naked mole-rat miRNA:mRNA target mapping.

Supplementary Table S4. KEGG pathway analysis.

Supplementary Table S5. GO term Molecular Function analysis.

Supplementary Table S6. GO term Cellular Components analysis.

Supplementary Table S7. GO term Biological Process analysis. 


\section{Chapter 5}

AMPK regulation in naked mole-rat muscle 


\title{
MicroRNA-mediated inhibition of AMPK coordinates tissue-specific downregulation of skeletal muscle metabolism in hypoxic naked mole-rats
}

\author{
Hanane Hadj-Moussa ${ }^{1}$, Sarah Chiasson ${ }^{2}$, Hang Cheng ${ }^{2, \dagger}$, Liam Eaton ${ }^{2, \dagger}$, Kenneth B. Storey ${ }^{1, \#}$ \\ and Matthew E. Pamenter ${ }^{2,3, \#}$ \\ ${ }^{1}$ Department of Biology, Carleton University, Ottawa, ON, Canada \\ 2 Biology Department, University of Ottawa, Ottawa, ON, Canada \\ ${ }^{3}$ Brain and Mind Research Institute, University of Ottawa, Ottawa, ON, Canada \\ \# Equal contribution \\ $\dagger$ Equal contribution
}

This study is published in the Journal of Experimental Biology:

Hadj-Moussa H, Chiasson S, Cheng H, Eaton L, Storey KB, \& Pamenter ME. (2021) MicroRNAmediated inhibition of AMPK coordinates tissue-specific downregulation of skeletal muscle metabolism in hypoxic naked mole-rats. Journal of Experimental Biology. 1;224(15): jeb242968. 


\subsection{Abstract}

Naked mole-rats reduce their metabolic requirements to tolerate severe hypoxia. However, the regulatory mechanisms that underpin this metabolic suppression have yet to be elucidated. 5' AMP-activated protein kinase (AMPK) is the cellular "master energy sensor" and we hypothesized that alterations in the AMPK pathway in naked mole-rat skeletal muscle would contribute to metabolic reorganization in hypoxia. To test this hypothesis, we exposed naked mole-rats to 4 hrs of normoxia $\left(21 \% \mathrm{O}_{2}\right)$ or severe hypoxia $\left(3 \% \mathrm{O}_{2}\right)$ while indirectly measuring whole animal metabolic rate and fuel preference. We then isolated skeletal muscle and assessed total protein levels and post-translational modification of AMPK, and downstream changes in key glucose and fatty acid metabolic proteins mediated by AMPK, including Acetyl-CoA carboxylase (ACC1), glycogen synthase (GS), and glucose transporters (GLUTs) 1 and 4 . We found that in hypoxic naked mole-rats (1) metabolic rate decreased $\sim 80 \%$ and fuel use switched to carbohydrate metabolism, and that (2) levels of activated phosphorylated AMPK and GS, and GLUT4 expression were downregulated, while ACC1 was unchanged. To explore the underlying regulatory mechanism of this hypometabolic state, we used RT-qPCR to examine 55 AMPKassociated microRNAs (miRNAs), which are short non-coding RNA post-transcriptional silencers. We identified changes in 10 hypoxia-induced miRNAs (three upregulated and seven downregulated), most of which are implicated in AMPK downregulation. Our results suggest that miRNA and post-translational mechanisms coordinately reduce AMPK activity and downregulate metabolism in naked mole-rat skeletal muscle during severe hypoxia. This novel mechanism may support tissue-specific prioritization of energy for more essential organs in severe hypoxia. 


\subsection{Introduction}

Eusocial East-African naked mole-rats (Heterocephalus glaber) live in crowded underground tunnel systems, wherein they putatively experience intermittent hypoxic conditions in their nesting chambers. Whereas the absolute levels of hypoxia within their underground burrow systems are poorly studied (Holtze et al., 2018), laboratory studies have demonstrated that naked mole-rats are remarkably hypoxia-tolerant (for an adult mammal), and can withstand minutes of anoxia, hours at $3 \% \mathrm{O}_{2}$, and days to weeks at 8-10\% $\mathrm{O}_{2}$ (Chung et al., 2016; Pamenter et al., 2015; Park et al., 2017). Naked mole-rats possess an impressive arsenal of adaptations that allow them to survive oxygen-deprivation. Perhaps most critically, and similar to other champions of hypoxia and anoxia-tolerance (Boutilier, 2001; Buck and Pamenter, 2006; Hochachka et al., 1996), naked molerats reduce their metabolic rate in oxygen-limiting environments (Pamenter et al., 2015), which presumably enables their energy demands to be met by less efficient anaerobic pathways (Pamenter et al., 2019). In support of this, naked mole-rat blood glucose levels are elevated during 4 hrs of progressively deeper hypoxia $\left(9-3 \% \mathrm{O}_{2}\right)$ (Pamenter et al., 2019), and anoxia (Park et al., 2017), tissue [ATP] are largely maintained, and respiratory exchange ratio (RER) calculations indirectly support a whole-animal metabolic fuel switch from primarily lipid-based metabolism in normoxia to carbohydrate-based metabolism in hypoxia (Pamenter et al., 2019). However, the cellular mechanisms that regulate this putative hypoxic metabolic fuel switch remain unknown.

Cells use various processes to regulate metabolism and balance the activation and suppression of energetically-expensive biological processes when ATP availability is limited. One of the primary initiators of such metabolic remodelling is 5'-AMP-activated proteins kinase (AMPK), which is a master cellular energy sensor. AMPK is activated by changes in the intracellular AMP:ATP ratio, such that breakdown of ATP to AMP favours activation of AMPK, which in turn 
modifies cellular energetics and metabolic fuel use (Hardie et al., 2012). For example, AMPK activation during low energy stress, such as occurs during hypoxia or anoxia, leads to a decrease in ATP-consuming anabolic pathways (e.g., fatty acid synthesis, which is gated by acetyl-CoA carboxylase 1 (ACC1)) (Hardie, 2007). Specifically, AMPK-mediated phosphorylation inhibits ACC1 activity, thereby inhibiting lipogenesis, and stimulates fatty acid oxidation to conserve and alter fuel use during metabolic rate suppression and environmental stress (Winder et al., 2003). This is typically associated with a stimulation of glucose uptake via glucose transporter proteins (GLUTs) in muscle, along with the inhibition of glycogen synthase (GS) to favor glycolysis (Kahn et al., 2005; Rider, 2016).

A key mechanism implicated in modulating cellular processes during hypoxia is microRNAs (miRNAs), which are short non-coding RNA transcripts. MiRNAs post-transcriptionally regulate gene expression temporarily through suppression of mRNA translation via transcript storage, or through permanent degradation, depending on the degree of miRNA complementary binding (O'Brien et al., 2018). MiRNAs are of particular interest in natural models of metabolic reorganization because they: (1) are reversible, (2) are rapidly acting, (3) are energetical inexpensive to synthesize, and (4) can target virtually all biological pathways (Hadj-Moussa and Storey, 2020). Studies on other hypoxia and anoxia-tolerant organisms have identified subsets of species- and tissue-specific oxygen-responsive miRNAs known as 'OxymiRs', which are involved in protective roles and energy reprioritization when physiological oxygen levels decline; however, it is also clear that there is not a unified miRNA response to oxygen limitation (Hadj-Moussa and Storey, 2020).

In the present study, we examined a potential role for AMPK in mediating metabolic rate suppression and the hypoxic fuel switch previously identified in freely behaving naked mole-rats 
and explored a potential regulatory role for miRNAs that target AMPK pathways. We hypothesized that naked mole-rat skeletal muscle would exhibit an AMPK-mediated fuel substrate switch to favour carbohydrate metabolism and enhance glycolysis, and predicted that this switch would be mediated by miRNA regulation of AMPK.

\subsection{Materials and Methodology}

\subsubsection{Animals.}

Naked mole-rats, Heterocephalus glaber Rüppell 1842, were group-housed in interconnected multi-cage systems at $30^{\circ} \mathrm{C}$ and $21 \% \mathrm{O}_{2}$ in $50 \%$ humidity with a $12 \mathrm{~h}$ dim light: $12 \mathrm{~h}$ dark cycle. Animals were fed fresh tubers, vegetables, fruit and Pronutro cereal supplement ad libitum. Animals were not fasted prior to experimental trials. All experimental procedures were approved by the University of Ottawa Animal Care Committee in accordance with the Animals for Research Act and by the Canadian Council on Animal Care. All experiments were performed during daylight working hours in the middle of the animals' 12L:12D light cycle. We examined miRNA and protein changes following in vivo hypoxic exposure in non-breeding naked mole-rats that were 12 years old. Non-breeding (subordinate) naked mole-rats do not undergo sexual development or express sexual hormones and thus we did not take sex into consideration when evaluating our results (Holmes et al., 2009).

\subsubsection{Whole Animal Respirometry.}

Naked mole-rats $(n=6)$ were individually placed, unrestrained, into a $450 \mathrm{~mL}$ plexiglass respirometer chamber, which was situated within a larger environmental chamber held at $30^{\circ} \mathrm{C}$. The chamber temperature was recorded every 2 secs using a custom designed thermocouple (range 
$29.8^{\circ} \mathrm{C}-30.2^{\circ} \mathrm{C}$ ). Animals were provided with a thin layer of corn cob bedding and the respirometer was continuously ventilated with gas mixtures set to the desired fractional gas composition by calibrated rotameters (Krohne, Duisburg, Germany). Inflowing gas was set at a flow rate of 100 $\mathrm{mL} / \mathrm{min}$, determined using a calibrated mass flow meter (Alicat Scientific, Tuscon, AZ, USA), and consistent with previous measurements in this species (Chung et al., 2016; Dzal et al., 2019). The excurrent gas was passed through a desiccant (Drierite, W.A. Hammond Drierite Co. Ltd., Xenia, $\mathrm{OH}$, USA) before entering the cells of the $\mathrm{O}_{2}$ and $\mathrm{CO}_{2}$ analyzers (FC-10 Oxygen Analyzer and CA-10 Carbon Dioxide Analyzer, Sable Systems), which were used to determine the gas concentrations of inspired and expired air. Before each trial the $\mathrm{O}_{2}$ and $\mathrm{CO}_{2}$ analyzers were calibrated using $100 \% \mathrm{~N}_{2}$, compressed air $\left(20.95 \% \mathrm{O}_{2}\right)$, and a span gas $\left(1.5 \% \mathrm{CO}_{2}\right.$; balance $\left.\mathrm{N} 2\right)$.

Prior to experimentation, animals were placed in the respirometry chamber for $1 \mathrm{hr}$ to familiarize them with their new surroundings. Oxygen consumption $\left(\mathrm{V}_{2}\right)$ and carbon dioxide production $\left(\dot{\mathrm{V} C O}_{2}\right)$ were then recorded for the next hour (normoxic control period). The incurrent gas was then switched to $3 \% \mathrm{O}_{2}\left(0.05 \% \mathrm{CO}_{2}\right.$, balance $\left.\mathrm{N}_{2}\right)$ and flow rates was increased to $1 \mathrm{~L} / \mathrm{min}$ for 10 mins to rapidly reduce the $\mathrm{O}_{2}$ content of the chamber to $3 \% \mathrm{O}_{2}$. The flow rate was then returned to $0.1 \mathrm{~L} / \mathrm{min}$ for the remaining $4 \mathrm{hrs}$ of hypoxic exposure. $\dot{\mathrm{V}} \mathrm{O}_{2}$ and $\dot{\mathrm{V}} \mathrm{CO}_{2}$ were measured during the last 30 mins (in three 10-min intervals) of each hour and these values were averaged for each animal. For 5 mins at the end of each hour, incurrent gas concentrations were measured by bypassing the experimental chamber and diverting air flow directly to the $\mathrm{O}_{2}$ and $\mathrm{CO}_{2}$ analyzers. Oxygen consumption and $\mathrm{CO}_{2}$ production rates were calculated using equations 10.6 and 10.7 in Lighton (2008), respectively:

$$
\dot{V} \mathrm{O}_{2}=\dot{V} \mathrm{i}\left[\left(\mathrm{FiO}_{2}-\mathrm{FeO}_{2}\right)-\mathrm{FeO}_{2}\left(\mathrm{FeCO}_{2}-\mathrm{FiCO}_{2}\right)\right] /\left(1-\mathrm{FeO}_{2}\right)
$$




$$
\dot{V} \mathrm{CO}_{2}=\dot{V} \mathrm{i}\left[\left(\mathrm{FeCO}_{2}-\mathrm{FiCO}_{2}\right)-\mathrm{FeCO}_{2}\left(\mathrm{FiO}_{2}-\mathrm{FeO}_{2}\right)\right] /\left(1-\mathrm{FeCO}_{2}\right)
$$

Where $\dot{\mathrm{V}} \mathrm{O}_{2}$ is $\mathrm{O}_{2}$ consumption rate $\left(\mathrm{ml} \mathrm{min}^{-1}\right)$ and $\dot{V} \mathrm{CO}_{2}$ is $\mathrm{CO}_{2}$ production rate $\left(\mathrm{ml} \mathrm{min}{ }^{-1}\right), \dot{V} \mathrm{i}$ is incurrent flow rate $\left(\mathrm{ml} \mathrm{min}^{-1}\right), \mathrm{FiO}_{2}$ and $\mathrm{FiCO}_{2}$ are fractional concentrations of incurrent $\mathrm{O}_{2}$ and $\mathrm{CO}_{2}$ of dry gas, respectively, and $\mathrm{FeO}_{2}$ and $\mathrm{FeCO}_{2}$ are fractional concentrations of excurrent $\mathrm{O}_{2}$ and $\mathrm{CO}_{2}$ of dry gas, respectively (Lighton, 2008). The respiratory exchange ratio (RER) was calculated by dividing $\dot{\mathrm{V}}_{\mathrm{CO} 2}$ by $\dot{\mathrm{V}}_{\mathrm{O} 2}$. All metabolic variables are reported at standard temperature, pressure, dry (STPD).

\subsubsection{Experimental Design for Tissue Collection.}

In other experiments, naked mole-rats were exposed to one of two treatment conditions: (1) normoxia ( 4 hrs in $21 \% \mathrm{O}_{2}$, balance $\mathrm{N}_{2} ; n=6$ ), or (2) acute hypoxia ( 4 hrs in $3 \% \mathrm{O}_{2}$, balance $\mathrm{N}_{2}$; $n=6$ ). Immediately following treatment, animals were killed by cervical dislocation followed by immediate decapitation. Temporalis muscles were rapidly dissected within 30 secs of euthanasia, and immediately flash frozen in liquid nitrogen and then stored at $-80{ }^{\circ} \mathrm{C}$ until analysis.

\subsubsection{Total RNA Isolation.}

Total RNA was isolated from normoxic and hypoxic naked mole-rat temporalis muscles (50 $\mathrm{mg} ; n=4$ each). RNA was isolated using Trizol-chloroform (Invitrogen; Cat\# 15596-018), as per the manufacturers instruction and as described in Hadj-Moussa et al., (2020). Precipitated RNA pellets were then resuspended in $50 \mu \mathrm{L}$ RNase-free water. RNA purity was determined by examining the $A_{260 / 280}$ ratio and concentration were assessed using a Take3 micro-volume plate and spectrophotometer (BioTek). RNA integrity was verified by running RNA samples on a $1 \%$ agarose gel with SYBR Green (Invitrogen; Cat\# S7563) and examining the presence of sharp bands for $28 \mathrm{~S}$ and $18 \mathrm{~S}$ rRNA. 


\subsubsection{MiRNA Quantification and Data Analysis.}

MiRNA sample preparation used $3 \mu \mathrm{g}$ of total RNA. An Epi-Bio PolyA tailing kit (Cat\# PAP5104H; Epicenter) was used for polyadenylation, as per the manufacturer's instructions. The polyadenylated RNA sample $(10 \mu \mathrm{L})$ was combined with $5 \mu \mathrm{L}$ of $250 \mathrm{pM}$ universal stem-loop adapter primer (Table S1). Stem-loop adapter ligation occurred on a thermocycler, heated to $95^{\circ} \mathrm{C}$ for $5 \mathrm{~min}$, incubated for $5 \mathrm{~min}$ at $65^{\circ} \mathrm{C}$, then chilled on ice. Reverse transcription was performed as per the manufacturer's instructions using the M-MLV reverse transcriptase (Cat\# 28025013; Invitrogen) and this PCR-program: $16^{\circ} \mathrm{C}$ for $30 \mathrm{~min}, 42^{\circ} \mathrm{C}$ for $30 \mathrm{~min}$, and $85^{\circ} \mathrm{C}$ for $5 \mathrm{~min}$.

Forward miRNA-specific primers were designed using naked mole-rat transcriptomic data and the universal reverse primer was designed using the described method (Table S1; (Biggar et al., 2014; Hadj-Moussa et al., 2021). MiRNA quantitative PCR (RT-qPCR) reactions were prepared as described by (Hadj-Moussa and Storey, 2020) and performed on a CFX Connect ${ }^{\mathrm{TM}}$ Real-Time PCR Detections System (Cat\# 1855201; BioRad) following MIQE guidelines (Bustin et al., 2009). The miRNA PCR program was as follows: an initial $3 \mathrm{~min}$ at $95^{\circ} \mathrm{C}$ and then 60 cycles of $95^{\circ} \mathrm{C}$ for $10 \mathrm{sec}$, and $60^{\circ} \mathrm{C}$ for $30 \mathrm{sec}$.

Differential miRNA expression levels $(n=3-4)$ were calculated using the $\Delta \Delta \mathrm{Cq}$ comparative method. Cycle threshold $(\mathrm{Cq})$ values were transformed to the $2^{-\mathrm{Cq}}$ form, to allow for the normalization of the miRNA of interest to the endogenous controls (Peltier and Latham, 2008; Schmittgen et al., 2008). The mean of Snord58a and Snord96a expression was found to be the most stable endogenous control between normoxic and hypoxic conditions. Data were collected and analyzed as mean relative expression (mean \pm SEM, where $n=3-4$ independent biological replicates from different animals at each sampling point). 


\subsubsection{Soluble Protein Isolation.}

Protein extraction was performed as per the manufacturer's instructions (EMD Millipore; 48630MAG). Briefly, $75 \mathrm{mg}$ of frozen tissue from normoxic and hypoxic naked mole-rats ( $n=6$ each) was homogenized in 1:5 (w/v) in 1X lysis buffer (EMD Millipore, 43-045). Lysis buffer was pre-chilled and combined with $1 \mathrm{mM} \mathrm{Na} \mathrm{VO}_{4}, 10 \mathrm{mM} \mathrm{NaF}, 10 \mathrm{mM} \beta$-Glycerophosphate, and 10 $\mu \mathrm{L} / \mathrm{mL}$ of protease inhibitor cocktail containing $104 \mathrm{mM}$ AEBSF, $80 \mu \mathrm{M}$ aprotinin, $4 \mathrm{mM}$ bestatin, 1.4 mM E-64, 2 mM leupeptin, and 1.5 mM pepstatin A (Bioshop, PIC001.1). Homogenates were placed on ice for $30 \mathrm{~min}$ and vortexed every $10 \mathrm{~min}$. Samples were then centrifuged at $14,000 \mathrm{x} \mathrm{g}$ for $20 \mathrm{~min}$ at $4{ }^{\circ} \mathrm{C}$. Soluble protein containing supernatants was collected, aliquoted, and stored at $-80{ }^{\circ} \mathrm{C}$ until later use.

Protein concentration were measured using the BioRad Protein Assay Kit II (Cat\# 5000002) as per the manufacturer's instructions. Protein concentrations were standardized using homogenization lysis buffer. Samples were then mixed 1:1 (v/v) with SDS buffer and 10\% $\beta$ mercaptoethanol. Samples were then boiled for $10 \mathrm{~min}$ and stored at $-20^{\circ} \mathrm{C}$ until use.

\subsubsection{Western Blotting and Data Analysis.}

Total protein homogenates $(25 \mu \mathrm{g})$ of each sample were loaded onto $6 \%-12 \%$ polyacrylamide gels and prepared with 5\% upper staking gels. For additional information on the SDS-PAGE and immunoblotting protocol, refer to (Hadj-Moussa and Storey, 2018). The following primary antibodies were used AMPK- $\alpha 1$ (Cat\# AF3197; R\&D Systems), AMPK- $\alpha 2$ (Cat\# AF2850; R\&D Systems), AMPK- $\beta$ (Cat\# GTX134594; GeneTex), phospho-AMPK (Thr172; p-AMPK) (Cat\# P01420-2; Boster), GLUT1 (Cat\# A6982; AbClonal), GLUT4 (Cat\# sc-7938; Santa Cruz), ACC (Cat\# GTX132081; GeneTex), phospho-ACC (Ser79; p-ACC) (Cat\# GTX133974; GeneTex), Glycogen synthase (Cat\# ab227270; AbCam), and phospho-Glycogen synthase (Ser641/645; p- 
GS) (Cat\# 07-817; Millipore-Sigma). After incubation membranes were incubated overnight in the target primary antibody, membranes were washed with TBST and incubated with the appropriate HRP-linked anti-rabbit, anti-mouse, or anti-goat secondary antibody (BioShop; 1:8000 $\mathrm{vv} / \mathrm{v}$ dilution in TBST) for $30 \mathrm{~min}$ at room temperature. Membranes were then washed in TBST and protein bands were visualized with enhanced chemiluminescence $\left(\mathrm{H}_{2} \mathrm{O}_{2}\right.$ and Luminol) on a ChemiGenius Bio-Imaging System (Syngene). Membranes were then stained with Coomassie blue $(0.25 \% \mathrm{w} / \mathrm{v}$ Coomassie Brilliant Blue, $7.5 \% \mathrm{v} / \mathrm{v}$ acetic acid, and $50 \% \mathrm{v} / \mathrm{v}$ methanol) to visualize total protein levels.

Relative protein abundance of chemiluminescent bands was quantified by densitometry using Gene Tools Software (Syngene). Minor variations in sample loading were corrected by standardizing the target protein band with the band intensity of a group of Coomassie Brilliant Blue-stained protein bands from each lane.

\subsubsection{Statistical Analysis.}

Respirometry data were analyzed using a 1-way repeated measures ANOVA with Tukey post hoc test. MiRNA relative expression between normoxic and hypoxic conditions was considered significant when an unpaired Student's $t$ test resulted in $p<0.05$ using the RBioplot statistical and graphing R package (Zhang and Storey, 2016). Western blot data were analyzed with an unpaired Student's t-test. Statistical analyses of whole-animal and immunoblot data were performed using commercial software (Prism v. 8.4.2, GraphPad Software Inc., CA, USA). All values are presented as mean $\pm \operatorname{SEM}$, where $p<0.05$ was the threshold for significance. 


\subsection{Results}

5.4.1 Naked mole-rats exhibit robust metabolic rate suppression and a fuel switch from mixed lipids to carbohydrates in acute hypoxia.

Relative to the normoxia baseline, naked mole-rats exhibited significant decreases of $63-80 \%$ in $\dot{\mathrm{V}}_{\mathrm{O} 2}$ and $\dot{\mathrm{V}}_{\mathrm{CO} 2}$ during $4 \mathrm{hrs}$ of acute and severe hypoxia exposure $\left(3 \% \mathrm{O}_{2}\right.$ inhaled; Figures 5.1A and 5.1B; $F_{1,5}=60.7, p<0.0005$ for $\dot{\mathrm{V}}_{\mathrm{O} 2}, F_{1,5}=121.2, p<0.0001$ for $\dot{\mathrm{V}}_{\mathrm{CO} 2} ; n=6$ for each). In addition, the RER was 0.67 in normoxia (Figure 5.1C, white circle), indicating that naked molerats predominately used lipid substrates as a metabolic fuel source. With the onset of severe hypoxia, naked mole-rats immediately exhibited a shift towards near total reliance on carbohydrates (RER of $\sim 1.02$ and 1.05 at 3 and 4 h of hypoxia, respectively; Fig. 5.1C, black squares; $\left.\mathrm{F}_{1,5}=9.6, p<0.0045 ; n=6\right)$.

\subsubsection{AMPK enzyme subunit expression and phosphorylation are altered during acute}

\section{hypoxia.}

Total and post-translational modification relative protein expression levels of regulatory AMPK subunits were examined using immunoblotting in normoxic and hypoxic naked mole-rat temporalis muscle. Protein levels of the AMPK $\alpha 1$ and $\beta$ subunits remained constant during hypoxia (Figure 5.2A; $p=0.46$ and 0.37 , respectively; $n=6$ for each). While levels of the AMPK $\alpha 2$ subunit was downregulated by $59 \%(t(10)=3.66, p=0.0044)$, and p-AMPK (Thr172) was downregulated by $58 \%(t(9)=2.9, p=0.017 ; n=6$ for each $)$. 


\subsubsection{Carbohydrate and lipid catabolic enzymes are reduced during hypoxia.}

The capacity for glucose uptake was examined by measuring the levels of GLUT1, which remained constant (Figure 5.3; $p=0.812 ; n=6$ for each), and GLUT4, which was downregulated by $45 \%$ in hypoxic naked mole-rat muscles $(t(10)=3.02, p=0.013 ; n=6$ for each). To examine if hypoxic muscles were mobilizing glucose reserves for synthesis into glycogen, levels of GS and its activated phosphorylated form p-GS (Ser641/645) were also examined. While levels of GS remained constant ( $p=0.44 ; n=6$ for each), the activated phosphorylated form was significantly downregulated during hypoxia by $35 \%(t(10)=2.26, p=0.047 ; n=6$ for each). Finally, the effect of AMPK on lipogenesis was investigated by examining levels of ACC1 and its activated phosphorylated form p-ACC1 (Ser79), both of which were unchanged by hypoxic conditions ( $p=$ 0.59 and 0.466 , respectively; $n=6$ for each).

A subset of AMPK-associated microRNAs were differentially regulated during hypoxia. Of the 55 AMPK-associated miRNAs that were assessed using RT-qPCR, ten were found to be significantly differently regulated during hypoxia as compared to normoxic values (Figure 5.4A). The three hypoxia upregulated microRNAs included miR-124-3p. miR-101a-3p, and miR-199a5p (Figure 5.4B). The seven hypoxia-downregulated microRNAs were: miR-137-3p, miR-200c3p, miR-214-3p. miR-223-3p. miR-296-5p, miR-325-3p, and miR-503-3p (Figure 5.4B). For a complete list of miRNAs quantified (mean relative expression $\pm \mathrm{SEM}$, and $\mathrm{p}$-values), please refer to Table S2.

\subsection{Discussion}

Severe hypoxia imposes significant restraints on aerobic energy production and most hypoxiatolerant species employ strategies to reduce systemic metabolic demands when oxygen is limited 
(Boutilier, 2001; Buck and Pamenter, 2006; Hochachka et al., 1996). Naked mole-rats likely experience intermittent and severe hypoxia in their natural habitat and are capable of robust metabolic depression in hypoxia, but avoid torpor and maintain physical activity, albeit at a reduced degree (Houlahan et al., 2018; Ilacqua et al., 2017; Kirby et al., 2018). A long-standing theory in the field posits that hypoxia-tolerant organisms prioritize energy to support the function of essential organs in hypoxia, such as the brain and heart, while reducing the energy demand of less hypoxia-sensitive tissues, such as skeletal muscle. However, a mechanism underlying tissuespecific metabolic reorganization has not been described. Here, we used a multi-pronged approach to examine naked mole-rat energy reprioritization in skeletal muscle during severe hypoxia. We report three salient findings: (1) metabolic rate decreases $>80 \%$ during 4 hrs of severe hypoxia and whole-animal fuel consumption switches from lipid-based to carbohydrate-based metabolism, consistent with previous studies in hypoxic naked mole-rats (Chung et al., 2016; Dzal et al., 2019; Ilacqua et al., 2017; Kirby et al., 2018; Pamenter et al., 2015; Pamenter et al., 2018; Pamenter et al., 2019; Vandewint et al., 2019). Concomitantly (2) key regulatory components of AMPK, and of downstream AMPK-regulated mediators of carbohydrate metabolism are downregulated, while (3) AMPK-regulating miRNAs are modified in a manner that is expected to reduce the overall activity of the AMPK pathway. Our findings imply a shift to an energy conserving state, with reduced capacity for carbohydrate metabolism in naked mole-rat skeletal muscle during severe hypoxia, and offer a mechanism via which tissue-specific hypometabolism may be achieved. 


\subsubsection{Hypoxia downregulates AMPK and downstream regulators of carbohydrate}

metabolism.

AMPK is a heterotrimer composed of a catalytic $\alpha$ subunit and two regulatory $\beta$ and $\gamma$ subunits (Ross et al., 2016). Whereas expression of the $\alpha 1$ - and $\beta$-subunits remain unchanged by hypoxia in naked mole-rat skeletal muscle, the $\alpha 2$ - subunit is downregulated, along with p-AMPK (Thr172), which is a key regulatory site on the $\alpha$ subunit. Phosphorylation at Thr172 can trigger an 100-fold increase in AMPK activity and promote a cellular switch from ATP consumption to ATP production (Suter et al., 2006). AMPK directly modulates components of cellular carbohydrate and fatty acid metabolism and the decrease in p-AMPK reported here suggests that naked mole-rat muscles are not inducing energy-consuming biosynthetic fatty acid or glucose oxidation in hypoxia.

Activation of AMPK, and specifically its phosphorylation at Thr172, stimulates GLUT activity to enhance glucose uptake into cells (Abbud et al., 2000; Zhang et al., 1999). AMPK also modulates cellular glycolytic activity by phosphorylating GS at Ser7 (Ha et al., 2015), with phosphorylation of GS leading to a catalytically inactive enzyme. Similarly, AMPK can alter fatty acid usage through its interaction with $\mathrm{ACC} 1$, which gates the fatty acid synthesis pathway by catalyzing the irreversible carboxylation of acetyl-CoA to produce malonyl-CoA, the building block for fatty acid chains (Hardie et al., 2012). Our findings that GLUT4 expression and GS phosphorylation both decrease in hypoxic skeletal muscle agree with our finding of decreased AMPK phosphorylation and suggest that AMPK mediates a hypoxic downregulation of glycolytic metabolism in this tissue. Conversely, we found no change in ACC1 or p-ACC1. When phosphorylated at Ser79 by AMPK, ACC1 activity is inhibited, which in turn halts fatty acid synthesis (Gusarova et al., 2009). The constant levels of p-ACC1 during hypoxia suggest that 
AMPK is not actively targeting this downstream target or phosphorylation during hypoxia, suggesting no change in fatty acid biosynthetic pathways.

These results differ from previous reports in skeletal muscle of hypoxia-intolerant mice, in which hypoxia activates AMPK, increases GLUT4 expression, and increases carbohydrate uptake into cells, whereas a dominant inhibitory mutant of AMPK prevented these changes in hypoxia (Mu et al., 2001). Indeed, AMPK is broadly activated as a result of energy imbalance during hypoxia, with evidence supporting this relationship in brain, liver, skeletal muscle, cardiomyocytes, alveolar and intestinal epithelial cells, and fibroblasts from various hypoxiaintolerant species (Dengler, 2020), along with downstream activation of glucose uptake into cells and altered fatty-acid metabolism, among other effects. Indeed, to the best of our knowledge, ours is the first report of AMPK being actively downregulated during hypoxia in any tissue of any species.

\subsubsection{AMPK-associated miRNAs are hypoxia-responsive in skeletal muscle.}

A key reason for the divergent response between naked mole-rat skeletal muscle and various tissues in hypoxia-intolerant species may be that ATP is not depleted during severe hypoxia in naked mole-rat skeletal muscle (Pamenter et al., 2019), as is generally the case during periods of oxygen scarcity (or anaerobic exercise). Given the absence of a cellular energy imbalance to alter AMPK activity in hypoxic skeletal muscle, and the atypical decrease in AMPK function in this tissue, we examined a potential novel role for miRNAs in negatively regulating AMPK in hypoxia. We found that the expression of most AMPK-associated microRNAs does not change in naked mole-rat skeletal muscle during hypoxia; however, 10 miRNAs that are associated with key regulatory aspects of AMPK and downstream AMPK-signalling were altered in our experiments. Of the three miRNAs that were upregulated in hypoxia, miR-124 is implicated in AMPK 
phosphorylation, as suppression of this miRNA increases p-AMPK protein levels (Gong et al., 2016; He et al., 2020), while overexpression of miR-124 is linked to a reduction in cell proliferation via G1-phase cell cycle arrest, which is an energy-expensive cellular process that can be halted during hypoxic energy-conserving states (Lang and Ling, 2012). In addition, miR-124 over-expression also decreases both cell growth and glucose consumption in cancer cells (Zhao et al., 2017), consistent with a downregulation of AMPK. Similarly, upregulated levels of miR-101a and miR-199a are associated with reductions in AMPK signalling (Li et al., 2020), and miR-101a can both directly and indirectly inactivate AMPK (Li et al., 2018; Liu et al., 2016). The seven microRNAs that were downregulated during hypoxia are also implicated in AMPK signalling, primarily downstream of direct regulation of the kinase, and play important roles in alleviating oxidative stress, autophagy, and apoptosis during hypoxia, or following anoxia/reoxygenation (da Cruz et al., 2018; Li et al., 2016; Sun et al., 2018; Tran et al., 2017; Zhao et al., 2020; Zhu et al., 2018). Intriguingly, downregulation of miR-223 is also associated with downregulation of GLUT4 protein expression (Lu et al., 2010), like our findings.

\subsubsection{A mechanism for tissue-specific energy prioritization in hypoxia?}

Importantly, naked mole-rats mobilize glucose from the liver to the blood during severe hypoxia (Pamenter et al., 2019). If muscle is not utilizing this carbohydrate fuel, then where is it going? One promising explanation is that this fuel is being prioritized for use by the brain, which is highly sensitive to energy depletion in hypoxia; by reducing glucose uptake and use by muscle cells, circulating glucose would remain available to more hypoxia-sensitive tissues. In support of this, we have previously reported that brain [ATP] falls in severe hypoxia (Pamenter et al., 2019), which is a canonical activator of AMPK. Furthermore, in a previous study we assayed changes in $200+$ miRNAs in naked mole-rat brain following acute in vivo hypoxia $\left(4 \mathrm{hrs}\right.$ at $\left.7 \% \mathrm{O}_{2}\right)$ (Hadj- 
Moussa et al., 2021). These miRNAs include 19 of the 55 AMPK-related transcripts that were assayed in the present study. Of these 19, six were significantly downregulated in hypoxic brain and none were upregulated $(p<0.10)$, including miR-124-3p $(p=0.084$; and which is upregulated in muscle), miR-140-3P $(p=0.069), \operatorname{miR}-140-5 p(p=0.033)$, miR-19b-3p $(p=0.046)$, and miR$301 \mathrm{a}-3 \mathrm{p}(p=0.016)$, all of which are unchanged in muscle. Notably, the downregulation of each of these 5 miRNAs in the brain correlates with increased AMPK activity (Bhardwaj et al., 2018; Gong et al., 2016; He et al., 2020; Sun et al., 2019; Yeon et al., 2019). In addition, miR-199a-5p and miR-223-3p, which were upregulated and downregulated in muscle, respectively (see above), are unchanged in brain (Hadj-Moussa et al., 2021). Finally, miR-325-3p is downregulated in both tissues, but the impact of this miRNA on AMPK function is unclear.

Taken together, these data support the hypothesis that AMPK is upregulated in naked molerat brain during hypoxia, in direct contrast to the changes observed in skeletal muscle. This relationship hints at a potential mechanism via which miRNAs may mediate tissue-specific metabolic fuel switches in hypoxic naked mole-rats, and support prioritization of limited energetic resources for more vulnerable and essential organs during severe hypoxia. Further experiments in the brain are warranted to explore hypoxia-mediated changes in AMPK phosphorylation and downstream changes in GLUTs and both glycolytic and fatty acid oxidation enzymes.

Interestingly, AMPK may similarly regulate tissue-specific metabolism in other hypoxiatolerant species, but the scarce information available presents a variable pattern. For example, AMPK is activated in goldfish (Carassius auratus) liver during 12 hrs of severe hypoxia, but is not altered in muscle, brain, heart, or gill (Jibb and Richards, 2008). Similarly, in anoxia-tolerant crucian carp (Carassius carassius) AMPK phosphorylation is slightly increased in the brain and heart following 10 days of hypoxia but is markedly increased following 10 days of anoxia 
(Stenslokken et al., 2008). In anoxia-tolerant red-eared slider turtles (Trachemys scripta elegans), AMPK is phosphorylated in white muscle and heart, but is unchanged in liver and red muscle following $20 \mathrm{hrs}$ of anoxia (Rider et al., 2009). While these examples demonstrate tissue-specific increases in AMPK-mediated metabolic function in other hypoxia-tolerant species, our finding of coordinated downregulation of AMPK-mediated energetics in skeletal muscle appears unique and also provides a potential regulatory mechanism.

\subsection{Conclusions}

Aerobic energy production is significantly impaired during periods of low-oxygen stress and anaerobic metabolism is less efficient and leads to the build-up of potentially deleterious endproducts, which must be catabolized via energetically expensive processes upon reoxygenation. For animals that live in intermittent hypoxia, metabolic downregulation is a more efficient and common strategy then upregulation of anaerobic pathways (Boutilier, 2001; Buck and Pamenter, 2006; Hochachka et al., 1996). However, across organ systems, oxygen availability and metabolic capacity must often be prioritized for key tissues, particularly the heart and brain, which must remain functional in hypoxia. Conversely, skeletal muscle, which often functions via anaerobic metabolism during intense exercise, is more tolerant of localized hypoxia and thus shutting down metabolism in this tissue offers a means to conserve and redirect energy towards more vital organs in severely hypoxic conditions. Although others have suggested that hypoxic species prioritize the energy demands of the heart and brain over those of the muscle, no previous study has demonstrated an inhibition of AMPK signalling during hypoxia in any tissue. Our results in naked mole-rat skeletal muscle, and comparison to previous analysis in hypoxic naked mole-rat brain, suggest that miRNA may offer such a mechanism and coordinate the AMPK-signalling axis to 
locally regulate metabolic demand at the tissue level, thereby driving metabolic reorganization to prioritize key organs during periods of severe hypoxia. 


\subsection{References}

Abbud W., Habinowski S., Zhang J.Z., Kendrew J., Elkairi F.S., Kemp B.E., Witters L.A., IsmailBeigi F. 2000 Stimulation of AMP-activated protein kinase (AMPK) is associated with enhancement of Glut1-mediated glucose transport. Arch Biochem Biophys 380(2), 347352.

Biggar K.K., Wu C.W., Storey K.B. 2014 High-throughput amplification of mature microRNAs in uncharacterized animal models using polyadenylated RNA and stem-loop reverse transcription polymerase chain reaction. Anal Biochem 462, 32-34.

Bhardwaj A., Singh H., Trinidad C.M., Albarracin C.T., Hunt K.K., Bedrosian I. 2018 The isomiR140-3p-regulated mevalonic acid pathway as a potential target for prevention of triple negative breast cancer. Breast Cancer Res 20(1), 150.

Boutilier R.G. 2001 Mechanisms of cell survival in hypoxia and hypothermia. The Journal of experimental biology 204, 3171-3181.

Buck L.T., Pamenter M.E. 2006 Adaptive responses of vertebrate neurons to anoxia--matching supply to demand. Respir Physiol Neurobiol 154(1-2), 226-240.

Bustin S.A., Benes V., Garson J.A., Hellemans J., Huggett J., Kubista M., Mueller R., Nolan T., Pfaffl M.W., Shipley G.L., et al. 2009 The MIQE guidelines: minimum information for publication of quantitative real-time PCR experiments. Clin Chem 55(4), 611-622.

Chung D., Dzal Y.A., Seow A., Milsom W.K., Pamenter M.E. 2016 Naked mole-rats exhibit metabolic but not ventilatory plasticity following chronic sustained hypoxia. Proc Biol Sci 283(1827).

da Cruz R.S., Carney E.J., Clarke J., Cao H., Cruz M.I., Benitez C., Jin L., Fu Y., Cheng Z., Wang Y., et al. 2018 Paternal malnutrition programs breast cancer risk and tumor metabolism in offspring. Breast Cancer Res 20(1), 99.

Dengler F. 2020 Activation of AMPK under Hypoxia: Many Roads Leading to Rome. Int J Mol Sci 21(7).

Dzal Y.A., Seow A., Borecky L.G., Chung D., Gill S.K.G., Milsom W.K., Pamenter M.E. 2019 Glutamatergic Receptors Modulate Normoxic but Not Hypoxic Ventilation and Metabolism in Naked Mole-rats. Front Physiol 10, 106.

Gong X., Wang H., Ye Y., Shu Y., Deng Y., He X., Lu G., Zhang S. 2016 miR-124 regulates cell apoptosis and autophagy in dopaminergic neurons and protects them by regulating AMPK/mTOR pathway in Parkinson's disease. Am J Transl Res 8(5), 2127-2137.

Gusarova G.A., Dada L.A., Kelly A.M., Brodie C., Witters L.A., Chandel N.S., Sznajder J.I. 2009 Alpha1-AMP-activated protein kinase regulates hypoxia-induced Na,K-ATPase endocytosis via direct phosphorylation of protein kinase C zeta. Mol Cell Biol 29(13), 3455-3464. 
Ha J., Guan K.L., Kim J. 2015 AMPK and autophagy in glucose/glycogen metabolism. Mol Aspects Med 46, 46-62.

Hadj-Moussa H., Storey K.B. 2018 Micromanaging freeze tolerance: the biogenesis and regulation of neuroprotective microRNAs in frozen brains. Cell Mol Life Sci 75(19), 3635-3647.

Hadj-Moussa H., Storey K.B. 2020 The OxymiR response to oxygen limitation: a comparative microRNA perspective. J Exp Biol 223(Pt 10).

Hadj-Moussa H., Pamenter M.E., Storey K.B. 2021 Hypoxic naked mole-rat brains use microRNA to coordinate hypometabolic fuels and neuroprotective defenses. J Cell Physiol 236(7), 5080-5097.

Hardie D.G. 2007 AMP-activated/SNF1 protein kinases: conserved guardians of cellular energy. Nat Rev Mol Cell Biol 8(10), 774-785.

Hardie D.G., Ross F.A., Hawley S.A. 2012 AMPK: a nutrient and energy sensor that maintains energy homeostasis. Nat Rev Mol Cell Biol 13(4), 251-262.

He Y., Yang Y., Liao Y., Xu J., Liu L., Li C., Xiong X. 2020 miR-140-3p Inhibits Cutaneous Melanoma Progression by Disrupting AKT/p70S6K and JNK Pathways through ABHD2. Mol Ther Oncolytics 17, 83-93.

Hochachka P.W., Buck L.T., Doll C.J., Land S.C. 1996 Unifying theory of hypoxia tolerance: molecular/metabolic defense and rescue mechanisms for surviving oxygen lack. Proc Natl Acad Sci U S A 93(18), 9493-9498.

Holmes M.M., Goldman B.D., Goldman S.L., Seney M.L., Forger N.G. 2009 Neuroendocrinology and sexual differentiation in eusocial mammals. Front Neuroendocrinol 30(4), 519-533.

Holtze S., Braude S., Lemma A., Koch R., Morhart M., Szafranski K., Platzer M., Alemayehu A., Goeritz F., Hildebrandt T.B. 2018 The microenvironment of naked mole-rat burrows in East Africa. African Journal of Ecology 56(2), 279-289.

Houlahan C.R., Kirby A.M., Dzal Y.A., Fairman G.D., Pamenter M.E. 2018 Divergent behavioural responses to acute hypoxia between individuals and groups of naked molerats. Comp Biochem Physiol B Biochem Mol Biol.

Ilacqua A.N., Kirby A.M., Pamenter M.E. 2017 Behavioural responses of naked mole-rats to acute hypoxia and anoxia. Biology letters 13(12).

Jibb L.A., Richards J.G. 2008 AMP-activated protein kinase activity during metabolic rate depression in the hypoxic goldfish, Carassius auratus. J Exp Biol 211(Pt 19), 3111-3122.

Kahn B.B., Alquier T., Carling D., Hardie D.G. 2005 AMP-activated protein kinase: ancient energy gauge provides clues to modern understanding of metabolism. Cell Metab 1(1), 1525. 
Kirby A.M., Fairman G.D., Pamenter M.E. 2018 Atypical behavioural, metabolic and thermoregulatory responses to hypoxia in the naked mole-rat (Heterocephalus glaber). Journal of Zoology 305(2), 106-115.

Lang Q., Ling C. 2012 MiR-124 suppresses cell proliferation in hepatocellular carcinoma by targeting PIK3CA. Biochem Biophys Res Commun 426(2), 247-252.

Li J., Li J., Wei T., Li J. 2016 Down-Regulation of MicroRNA-137 Improves High GlucoseInduced Oxidative Stress Injury in Human Umbilical Vein Endothelial Cells by UpRegulation of AMPKalpha1. Cell Physiol Biochem 39(3), 847-859.

Li Y., Wang S., Gao X., Zhao Y., Li Y., Yang B., Zhang N., Ma L. 2018 Octreotide Alleviates Autophagy by Up-Regulation of MicroRNA-101 in Intestinal Epithelial Cell Line Caco-2. Cell Physiol Biochem 49(4), 1352-1363.

Li Y., Luan Y., Li J., Song H., Li Y., Qi H., Sun B., Zhang P., Wu X., Liu X., et al. 2020 Exosomal miR-199a-5p promotes hepatic lipid accumulation by modulating MST1 expression and fatty acid metabolism. Hepatol Int 14(6), 1057-1074.

Lighton J. 2008 Measuring metabolic rates: a manual for scientists. Oxford, Oxford University Press.

Liu P., Ye F., Xie X., Li X., Tang H., Li S., Huang X., Song C., Wei W., Xie X. 2016 mir-101-3p is a key regulator of tumor metabolism in triple negative breast cancer targeting AMPK. Oncotarget 7(23), 35188-35198.

Lu H., Buchan R.J., Cook S.A. 2010 MicroRNA-223 regulates Glut4 expression and cardiomyocyte glucose metabolism. Cardiovasc Res 86(3), 410-420.

Mu J., Brozinick J.T., Jr., Valladares O., Bucan M., Birnbaum M.J. 2001 A role for AMP-activated protein kinase in contraction- and hypoxia-regulated glucose transport in skeletal muscle. Mol Cell 7(5), 1085-1094.

O'Brien J., Hayder H., Zayed Y., Peng C. 2018 Overview of MicroRNA Biogenesis, Mechanisms of Actions, and Circulation. Front Endocrinol (Lausanne) 9, 402.

Pamenter M.E., Dzal Y.A., Milsom W.K. 2015 Adenosine receptors mediate the hypoxic ventilatory response but not the hypoxic metabolic response in the naked mole-rat during acute hypoxia. Proc Biol Sci 282(1800), 20141722.

Pamenter M.E., Lau G.Y., Richards J.G., Milsom W.K. 2018 Naked mole-rat brain mitochondria electron transport system flux and $\mathrm{H}(+)$ leak are reduced during acute hypoxia. J Exp Biol 221(Pt 4).

Pamenter M.E., Dzal Y.A., Thompson W.A., Milsom W.K. 2019 Do naked mole-rats accumulate a metabolic acidosis or an oxygen debt in severe hypoxia? J Exp Biol 222(3). 
Park T.J., Reznick J., Peterson B.L., Blass G., Omerbasic D., Bennett N.C., Kuich P.H.J.L., Zasada C., Browe B.M., Hamann W., et al. 2017 Fructose-driven glycolysis supports anoxia resistance in the naked mole-rat. Science 356(6335), 305-308.

Peltier H.J., Latham G.J. 2008 Normalization of microRNA expression levels in quantitative RTPCR assays: identification of suitable reference RNA targets in normal and cancerous human solid tissues. RNA 14(5), 844-852.

Rider M.H., Hussain N., Dilworth S.M., Storey K.B. 2009 Phosphorylation of translation factors in response to anoxia in turtles, Trachemys scripta elegans: role of the AMP-activated protein kinase and target of rapamycin signalling pathways. Mol Cell Biochem 332(1-2), 207-213.

Rider M.H. 2016 Role of AMP-activated protein kinase in metabolic depression in animals. J Comp Physiol B 186(1), 1-16.

Ross F.A., MacKintosh C., Hardie D.G. 2016 AMP-activated protein kinase: a cellular energy sensor that comes in 12 flavours. FEBS J 283(16), 2987-3001. (doi:10.1111/febs.13698).

Schmittgen T.D., Livak K.J. 2008 Analyzing real-time PCR data by the comparative C(T) method. Nat Protoc 3(6), 1101-1108.

Stenslokken K.O., Ellefsen S., Stecyk J.A., Dahl M.B., Nilsson G.E., Vaage J. 2008 Differential regulation of AMP-activated kinase and AKT kinase in response to oxygen availability in crucian carp (Carassius carassius). Am J Physiol Regul Integr Comp Physiol 295(6), R1803-1814.

Sun T., Li M.Y., Li P.F., Cao J.M. 2018 MicroRNAs in Cardiac Autophagy: Small Molecules and Big Role. Cells 7(8).

Sun Y., Song Y., Liu C., Geng J. 2019 LncRNA NEAT1-MicroRNA-140 axis exacerbates nonalcoholic fatty liver through interrupting AMPK/SREBP-1 signaling. Biochem Biophys Res Commun 516(2), 584-590.

Suter M., Riek U., Tuerk R., Schlattner U., Wallimann T., Neumann D. 2006 Dissecting the role of 5'-AMP for allosteric stimulation, activation, and deactivation of AMP-activated protein kinase. J Biol Chem 281(43), 32207-32216.

Tran M., Lee S.M., Shin D.J., Wang L. 2017 Loss of miR-141/200c ameliorates hepatic steatosis and inflammation by reprogramming multiple signaling pathways in NASH. JCI Insight 2(21).

Vandewint A.L., Zhu-Pawlowsky A.J., Kirby A., Tattersall G.J., Pamenter M.E. 2019 Evaporative cooling and vasodilation mediate thermoregulation in naked mole-rats during normoxia but not hypoxia. J Therm Biol 84, 228-235. 
Winder W.W., Hardie D.G., Mustard K.J., Greenwood L.J., Paxton B.E., Park S.H., Rubink D.S., Taylor E.B. 2003 Long-term regulation of AMP-activated protein kinase and acetyl-CoA carboxylase in skeletal muscle. Biochem Soc Trans 31(Pt 1), 182-185.

Yeon M., Lee S., Lee J.E., Jung H.S., Kim Y., Jeoung D. 2019 CAGE-miR-140-5p-Wnt1 Axis Regulates Autophagic Flux, Tumorigenic Potential of Mouse Colon Cancer Cells and Cellular Interactions Mediated by Exosomes. Frontiers in oncology 9, 1240.

Zhang J., Storey K.B. 2016 RBioplot: an easy-to-use R pipeline for automated statistical analysis and data visualization in molecular biology and biochemistry. PeerJ 4, e2436.

Zhang J.Z., Behrooz A., Ismail-Beigi F. 1999 Regulation of glucose transport by hypoxia. American journal of kidney diseases : the official journal of the National Kidney Foundation 34(1), 189-202.

Zhao T., Qiu Z., Gao Y. 2020 MiR-137-3p exacerbates the ischemia-reperfusion injured cardiomyocyte apoptosis by targeting KLF15. Naunyn Schmiedebergs Arch Pharmacol 393(6), 1013-1024.

Zhao X., Lu C., Chu W., Zhang B., Zhen Q., Wang R., Zhang Y., Li Z., Lv B., Li H., et al. 2017 MicroRNA-124 suppresses proliferation and glycolysis in non-small cell lung cancer cells by targeting AKT-GLUT1/HKII. Tumour Biol 39(5), 1010428317706215.

Zhu W., Lei J., Bai X., Wang R., Ye Y., Bao J. 2018 MicroRNA-503 regulates hypoxia-induced cardiomyocytes apoptosis through PI3K/Akt pathway by targeting IGF-1R. Biochem Biophys Res Commun 506(4), 1026-1031. 

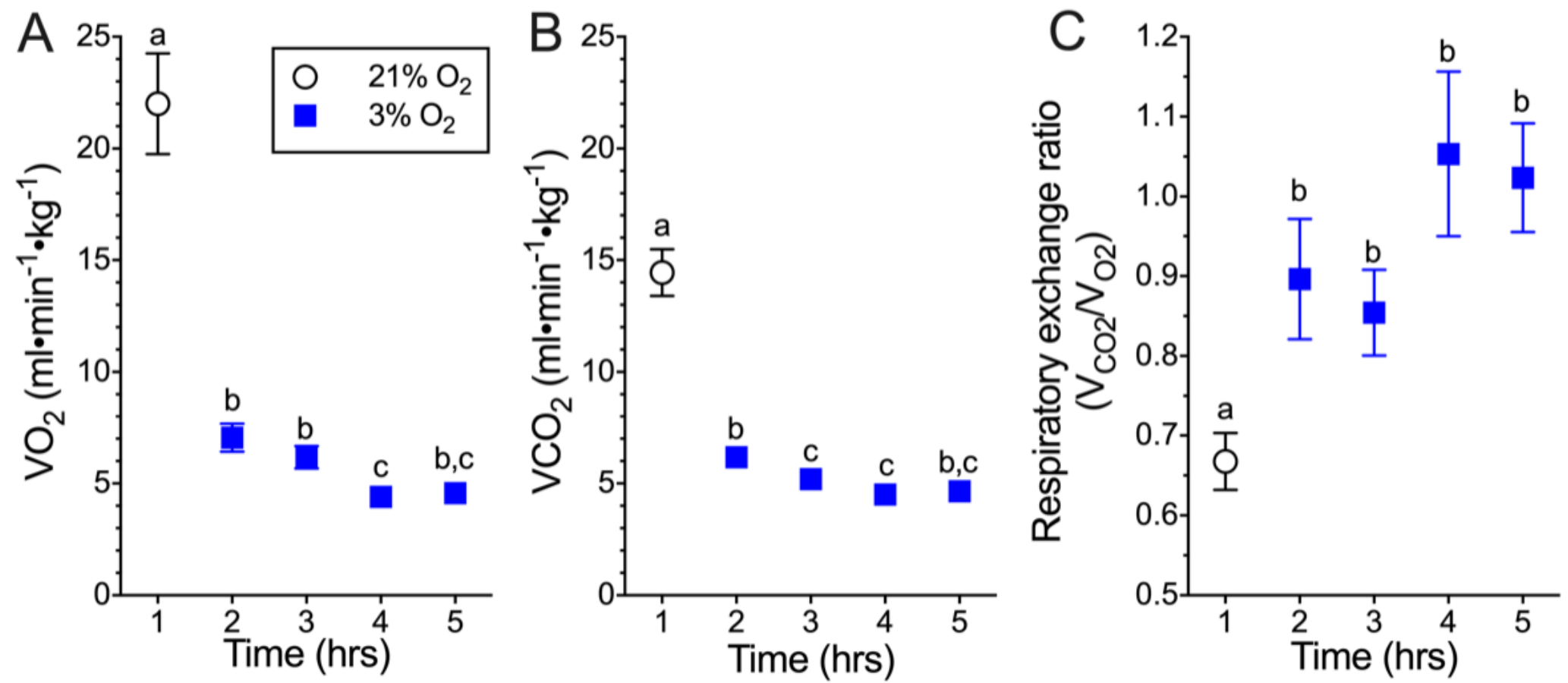

Figure 5.1. Naked mole-rats exhibit metabolic rate suppression and a hypoxic fuel shift from lipids to carbohydrates in acute hypoxia. Summaries of oxygen consumption rate $\left(\dot{\mathbf{V}}_{\mathrm{O} 2} ; \mathbf{A}\right)$, carbon dioxide production rate $\left(\dot{\mathbf{V}}_{\mathrm{CO} 2} ; \mathbf{B}\right)$, and respiratory exchange ratios (RER; $\left.\dot{\mathbf{V}}_{\mathrm{CO} 2} / \dot{\mathbf{V}}_{\mathrm{O} 2} ; \mathbf{C}\right)$ from 6 naked mole-rats exposed to $1 \mathrm{hr}$ of normoxia $\left(21 \% \mathrm{O}_{2}\right.$; white circles) and then 4 hrs of hypoxia $\left(3 \% \mathrm{O}_{2}\right.$; black squares). Data are presented as mean \pm SEM. Significant differences are indicated by different letters. $p<0.05,1-$ way ANOVA with Tukey's post-test. 


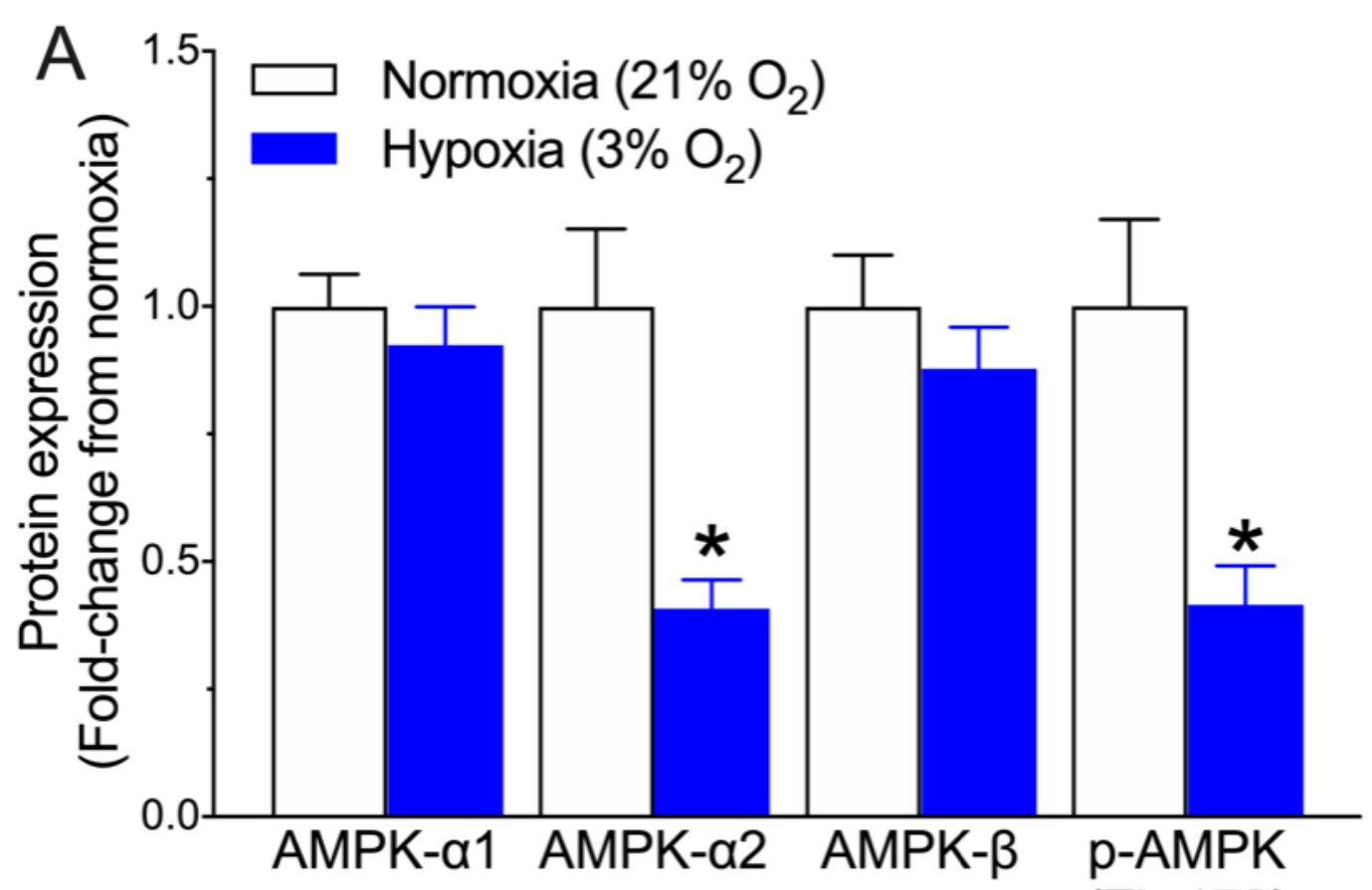

B

(Thr172)
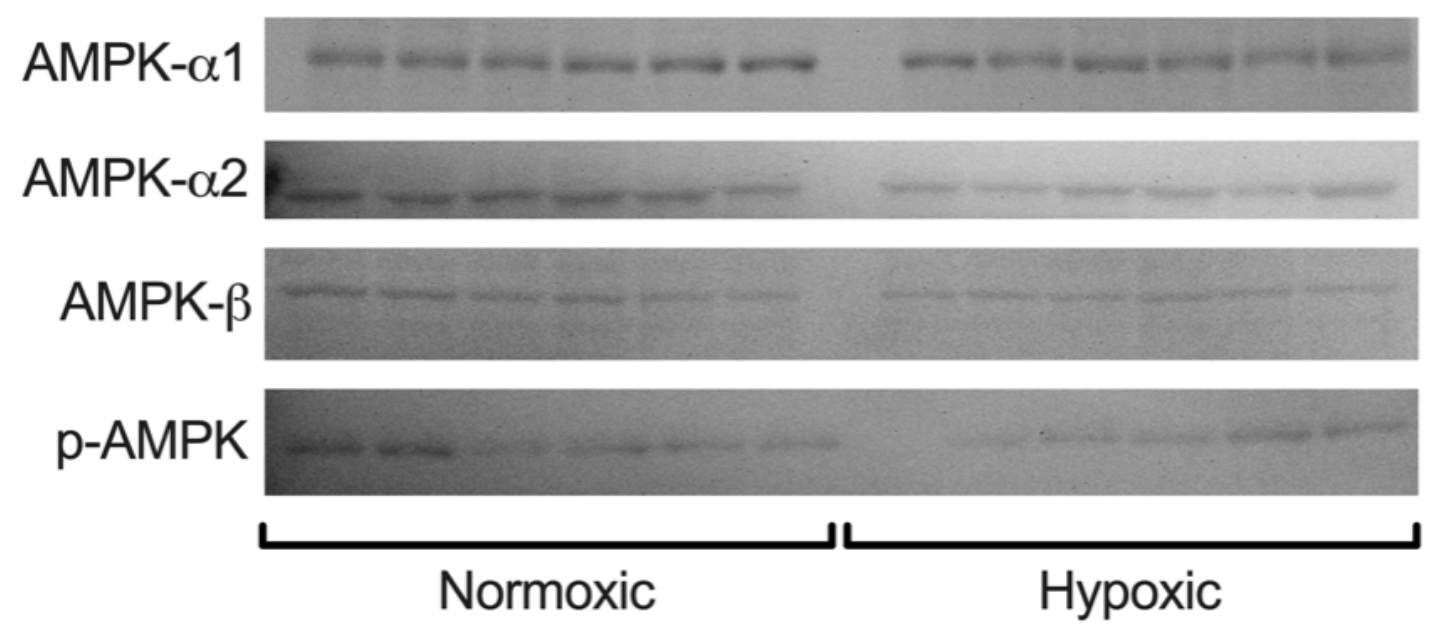

Figure 5.2. 5'-AMP-Kinase (AMPK) function is downregulated in skeletal muscle after acute hypoxia. Relative AMPK protein expression levels in normoxic and hypoxia naked molerat temporalis muscle. (A) Summary of AMPK subunits $(\alpha 1, \alpha 2, \beta)$ and phosphorylated form of p-AMPK (Thr172) protein expression. (B) Images of immunoblot protein expression from (A). Data are presented as mean \pm SEM from $n=5-6$ independent biological replicates for each condition. Significant differences from normoxic controls are indicated by asterisks $(*), p<0.05$, unpaired student's t-test. 

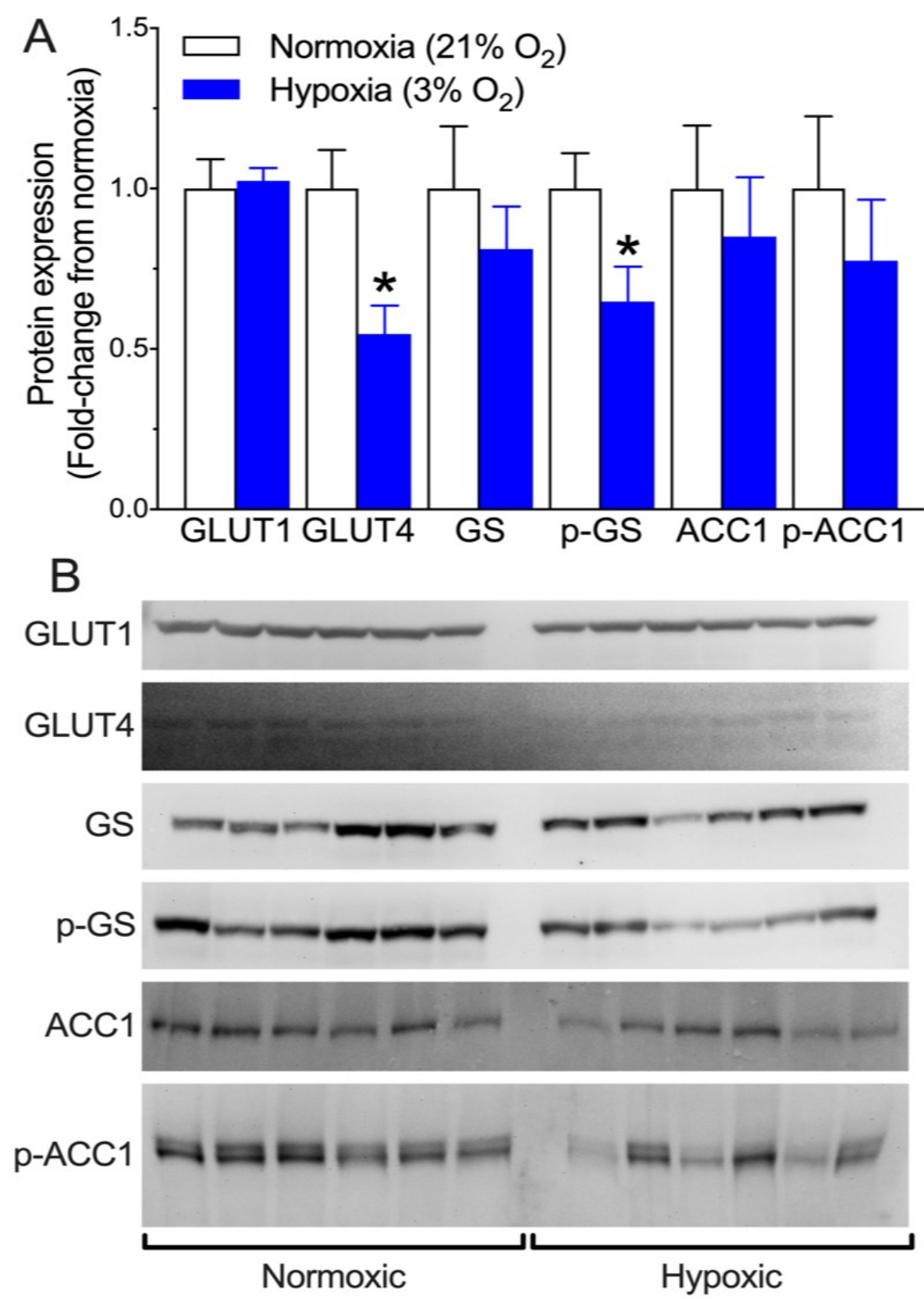

Figure 5.3. Glucose metabolism is downregulated in skeletal muscle after acute hypoxia. Relative protein expression levels in normoxic and hypoxic naked mole-rat temporalis muscle of glucose transporters (GLUT1 and GLUT4), glycogen synthase (GS) and its phosphorylated form p-GS (Ser641/645), and acetyl-CoA carboxylase1 (ACC1) and its phosphorylated form p-ACC1 (Ser79). (A) Summary of protein expression. (B) Images of immunoblot protein expression from (A). Data are presented as mean \pm SEM from $n=6$ independent biological replicates for each condition. Significant differences from normoxic controls are indicated by asterisks $(*) . p<0.05$, unpaired student's t-test. 

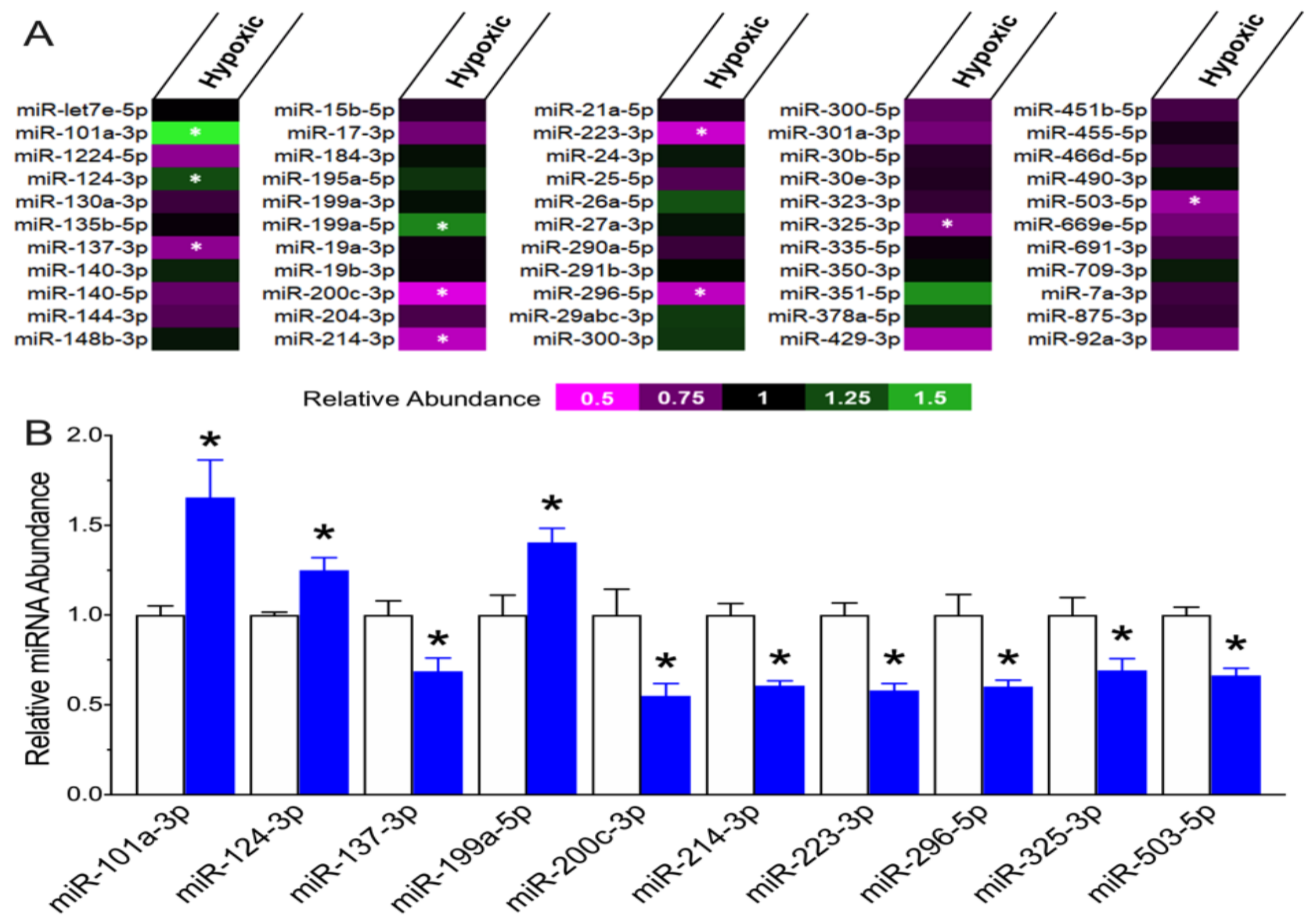

Figure 5.4. 5'-AMP-Kinase (AMPK)-regulating miRNAs are altered in acute hypoxia. (A) Heatmap of the relative expression levels of 55 AMPK-associated miRNAs in naked mole-rat temporalis muscle tissue. MiRNA expression was measured in 4 hr hypoxia relative to normoxic conditions using quantitative PCR (qPCR). (B) MiRNA expression of significantly changed hypoxia-induced miRNAs in temporalis muscles of naked mole-rats $(p<0.05)$. MiRNA expression was measured in 4 hrs hypoxia relative to normoxic conditions using quantitative PCR (qPCR). Data are means \pm SEM from $n=$ of $3-4$ independent biological replicates for each condition. Significant differences from normoxic controls are indicated by asterisks $(*) . p<0.05$, unpaired student's t-test. Refer to Table S2 for the relative expression \pm SEM values of all 55 miRNAs. 


\subsection{Supplementary material}

The data supporting this article is present in the figures and additional information is below:

Supplementary Table S1. MicroRNA primers used for naked mole-rat RT-qPCR analyses.

Supplementary Table S2. List of 55 miRNA relative expression levels measured in muscle tissue under hypoxic stress, relative to normoxic conditions using RT-qPCR. Data are means \pm SEM from $n=$ of $3-4$ independent biological replicates for each condition. 
Supplementary Table S1. MicroRNA primers used for naked mole-rat RT-qPCR analyses.

\begin{tabular}{|c|c|}
\hline Target & Primer Sequence $\left(5^{\prime}-3^{\prime}\right)$ \\
\hline $\operatorname{miR}-101 a-3 p$ & ACACTCCAGCTGGGTACAGTACTGTGATA \\
\hline $\operatorname{miR}-1224-5 p$ & ACACTCCAGCTGGGGTGAGGACTGGGGAG \\
\hline $\operatorname{miR}-124-3 p$ & ACACTCCAGCTGGGTAAGGCACGCGGTGA \\
\hline $\operatorname{miR}-130 a-3 p$ & ACACTCCAGCTGGGCAGTGCAATGTTAAA \\
\hline $\operatorname{miR}-135 b-5 p$ & ACACTCCAGCTGGGTATGGCTTTTCATTC \\
\hline miR-137-3p & ACACTCCAGCTGGGTTATTGCTTAAGAAT \\
\hline $\operatorname{miR}-140-3 p$ & ACACTCCAGCTGGGTACCACAGGGTAGAA \\
\hline $\operatorname{miR}-140-5 p$ & ACACTCCAGCTGGGCAGTGGTTTTACCCT \\
\hline miR-144-3p & ACACTCCAGCTGGGTACAGTATAGATGAT \\
\hline $\operatorname{miR}-148 b-3 p$ & ACACTCCAGCTGGGTCAGTGCATCACAGA \\
\hline $\operatorname{miR}-15 b-5 p$ & ACACTCCAGCTGGGTAGCAGCACATCATG \\
\hline miR-17-3p & ACACTCCAGCTGGGACTGCAGTGAGGGCA \\
\hline miR-184-3p & ACACTCCAGCTGGGTGGACGGAGAACTGA \\
\hline $\operatorname{miR}-195 a-5 p$ & ACACTCCAGCTGGGTAGCAGCACAGAAAT \\
\hline $\operatorname{miR}-199 a-3 p$ & ACACTCCAGCTGGGACAGTAGTCTGCACA \\
\hline $\operatorname{miR}-199 a-5 p$ & ACACTCCAGCTGGGCCCAGTGTTCAGACT \\
\hline miR-19a-3p & ACACTCCAGCTGGGTGTGCAAATCTATGC \\
\hline miR-19b-3p & ACACTCCAGCTGGGTGTGCAAATCCATGC \\
\hline $\operatorname{miR}-200 c-3 p$ & ACACTCCAGCTGGGTAATACTGCCGGGTA \\
\hline miR-204-3p & ACACTCCAGCTGGGGCTGGGAAGGCAAAG \\
\hline miR-214-3p & ACACTCCAGCTGGGACAGCAGGCACAGAC \\
\hline miR-21a-5p & ACACTCCAGCTGGGTAGCTTATCAGACTG \\
\hline miR-223-3p & ACACTCCAGCTGGGTGTCAGTTTGTCAAA \\
\hline miR-24-3p & ACACTCCAGCTGGGTGGCTCAGTTCAGCA \\
\hline miR-25-5p & ACACTCCAGCTGGGAGGCGGAGACTTGGG \\
\hline miR-26a-5p & ACACTCCAGCTGGGTTCAAGTAATCCAGG \\
\hline $\operatorname{miR}-27 a-3 p$ & ACACTCCAGCTGGGTTCACAGTGGCTAAG \\
\hline miR-290a-5p & ACACTCCAGCTGGGACTCAAACTATGGGG \\
\hline miR-291b-3p & ACACTCCAGCTGGGAAAGTGCATCCATTT \\
\hline miR-296-5p & ACACTCCAGCTGGGAGGGCCCCCCCTCAA \\
\hline miR-29abc-3p & ACACTCCAGCTGGGTAGCACCATCTGAAA \\
\hline miR-300-3p & ACACTCCAGCTGGGTATGCAAGGGCAAGC \\
\hline miR-300-5p & ACACTCCAGCTGGGTTGAAGAGAGGTTAT \\
\hline miR-301a-3p & ACACTCCAGCTGGGCAGTGCAATAGTATT \\
\hline miR-30b-5p & ACACTCCAGCTGGGTGTAAACATCCTACA \\
\hline miR-30e-3p & ACACTCCAGCTGGGCTTTCAGTCGGATGT \\
\hline miR-323-3p & ACACTCCAGCTGGGCACATTACACGGTCG \\
\hline miR-325-3p & ACACTCCAGCTGGGTTTATTGAGCACCTC \\
\hline miR-335-5p & ACACTCCAGCTGGGTCAAGAGCAATAACG \\
\hline miR-350-3p & ACACTCCAGCTGGGTTCACAAAGCCCATA \\
\hline miR-351-5p & ACACTCCAGCTGGGTCCCTGAGGAGCCCT \\
\hline
\end{tabular}




\begin{tabular}{|ll|}
\hline Target & Primer Sequence (5' - 3') \\
\hline miR-378a-5p & ACACTCCAGCTGGGCTCCTGACTCCAGGT \\
miR-429-3p & ACACTCCAGCTGGGTATACTGTCTGGTA \\
miR-451b-5p & ACACTCCAGCTGGGTGGGAGCAGCAAGAG \\
miR-455-5p & ACACTCCAGCTGGGTATGTGCCTTTGGAC \\
miR-466d-5p & ACACTCCAGCTGGGTGTGTGTGCGTACAT \\
miR-490-3p & ACACTCCAGCTGGGCAACCTGGAGGACTC \\
miR-503-5p & ACACTCCAGCTGGGTAGCAGCGGGAACAG \\
miR-669e-5p & ACACTCCAGCTGGGTGTCTTGTGTGTGCA \\
miR-691-3p & ACACTCCAGCTGGGATTCCTGAAGAGAGG \\
miR-709-3p & ACACTCCAGCTGGGGGAGGCAGAGGCAGG \\
miR-7a-3p & ACACTCCAGCTGGGCAACAAATCACAGTC \\
miR-875-3p & ACACTCCAGCTGGGCCTGAAAATACTGAG \\
miR-92a-3p & ACACTCCAGCTGGGTATTGCACTTGTCCC \\
miR-let7e-5p & ACACTCCAGCTGGGTGAGGTAGGAGGTTG \\
\hline snord58a & ACACTCCAGCTGGGTCTTAGGACACCTTT \\
snord96a & ACACTCCAGCTGGGATCCTAGTGATGACAAG \\
Universal Primer (reverse) & ACCGGTTCTCGTCCGATCACCGAAGT \\
miRNA Stem-loop Adapter & CTCACAGTACGTTGGTATCCTTGTGATGTTCGATGCCATATTGTA \\
\hline
\end{tabular}


Supplementary Table S2. List of 55 miRNA relative expression levels measured in muscle tissue under hypoxic stress, relative to normoxic conditions using RT-qPCR. Data are means $\pm \mathrm{SEM}$ from $n=$ of $3-4$ independent biological replicates for each condition.

\begin{tabular}{|c|c|c|c|}
\hline \multirow{2}{*}{ microRNA } & \multicolumn{3}{|c|}{ Hypoxia } \\
\hline & Relative expression & SEM & $p$-value \\
\hline miR-101a-3p & 1.655 & 0.2089 & $* 0.0473$ \\
\hline miR-1224-5p & 0.685 & 0.0784 & 0.0948 \\
\hline miR-124-3p & 1.251 & 0.0706 & *0.0344 \\
\hline miR-130a-3p & 0.851 & 0.0967 & 0.2474 \\
\hline miR-135b-5p & 0.985 & 0.2235 & 0.9521 \\
\hline miR-137-3p & 0.687 & 0.0737 & $* 0.0282$ \\
\hline miR-140-3p & 1.123 & 0.1055 & 0.3485 \\
\hline miR-140-5p & 0.763 & 0.1084 & 0.1137 \\
\hline miR-144-3p & 0.799 & 0.0294 & 0.4087 \\
\hline $\operatorname{miR}-148 b-3 p$ & 1.074 & 0.1122 & 0.5762 \\
\hline miR-15b-5p & 0.910 & 0.0789 & 0.6499 \\
\hline $\operatorname{miR}-17-3 p$ & 0.738 & 0.0510 & 0.0950 \\
\hline miR-184-3p & 1.050 & 0.2413 & 0.8537 \\
\hline $\operatorname{miR}-195 a-5 p$ & 1.180 & 0.1203 & 0.3693 \\
\hline miR-199a-3p & 1.049 & 0.0710 & 0.7276 \\
\hline miR-199a-5p & 1.405 & 0.0793 & ${ }^{*} 0.0276$ \\
\hline miR-19a-3p & 0.965 & 0.0949 & 0.8478 \\
\hline miR-19b-3p & 0.970 & 0.0573 & 0.7828 \\
\hline $\operatorname{miR}-200 c-3 p$ & 0.550 & 0.0694 & $* 0.0437$ \\
\hline miR-204-3p & 0.820 & 0.0899 & 0.2857 \\
\hline miR-214-3p & 0.607 & 0.0266 & $* 0.0046$ \\
\hline miR-21a-5p & 0.934 & 0.0673 & 0.5478 \\
\hline miR-223-3p & 0.581 & 0.0381 & $* 0.0181$ \\
\hline miR-24-3p & 1.081 & 0.0679 & 0.4440 \\
\hline miR-25-5p & 0.804 & 0.0940 & 0.1977 \\
\hline miR-26a-5p & 1.271 & 0.1190 & 0.1037 \\
\hline miR-27a-3p & 1.066 & 0.1086 & 0.6301 \\
\hline miR-290a-5p & 0.856 & 0.1327 & 0.6280 \\
\hline miR-291b-3p & 1.021 & 0.0867 & 0.8914 \\
\hline miR-296-5p & 0.603 & 0.0353 & $* 0.0346$ \\
\hline $\operatorname{miR}-29 a / b / c-3 p$ & 1.199 & 0.2115 & 0.4920 \\
\hline miR-300-3p & 1.186 & 0.1842 & 0.3951 \\
\hline miR-300-5p & 0.779 & 0.0923 & 0.0969 \\
\hline miR-301a-3p & 0.732 & 0.1116 & 0.1950 \\
\hline miR-30b-5p & 0.897 & 0.0539 & 0.2828 \\
\hline
\end{tabular}




\begin{tabular}{|lccc|}
\hline \multirow{2}{*}{ microRNA } & \multicolumn{3}{c|}{ Hypoxia } \\
\cline { 2 - 4 } & Relative expression & SEM & -value \\
\hline miR-30e-3p & 0.917 & 0.1219 & 0.7077 \\
miR-323-3p & 0.870 & 0.0912 & 0.4357 \\
miR-325-3p & 0.692 & 0.0658 & $* 0.0459$ \\
miR-335-5p & 0.972 & 0.1153 & 0.8325 \\
miR-350-3p & 1.046 & 0.0683 & 0.8154 \\
miR-351-5p & 1.431 & 0.1632 & 0.0721 \\
miR-378a-5p & 1.114 & 0.1120 & 0.4729 \\
miR-429-3p & 0.637 & 0.1259 & 0.0958 \\
miR-451b-5p & 0.831 & 0.0988 & 0.2450 \\
miR-455-5p & 0.934 & 0.2353 & 0.8101 \\
miR-466d-5p & 0.857 & 0.0536 & 0.3410 \\
miR-490-3p & 1.056 & 0.1966 & 0.8188 \\
miR-503-5p & 0.664 & 0.0398 & $* 0.0015$ \\
miR-669e-5p & 0.736 & 0.0707 & 0.0772 \\
miR-691-3p & 0.828 & 0.0786 & 0.2403 \\
miR-709-3p & 1.097 & 0.1151 & 0.8053 \\
miR-7a-3p & 0.841 & 0.0901 & 0.3821 \\
miR-875-3p & 0.865 & 0.0624 & 0.4943 \\
miR-92a-3p & 0.711 & 0.0638 & 0.0720 \\
miR-let7e-5p & 0.997 & 0.0901 & 0.9755 \\
\hline
\end{tabular}




\section{Chapter 6}

\section{General Discussion}




\subsection{Naked mole-rats and hypoxia tolerance}

Prolonged exposure to limited oxygen can be lethal. Investigating the biological consequences of oxygen-deprivation in a hypoxia tolerant mammalian model can provide us with a better understanding and novel insights that could be applied to alleviate the pathogenesis of ischemic insults, such as experienced during stroke, or to better tolerate the hypoxia of high-altitude, and boost recovery. Adaptations for hypoxia and anoxia tolerance have been, and continue to be, characterized in insects, fish, amphibians, and reptiles (Fago and Jensen, 2015; Krivoruchko and Storey, 2015; Storey, 2004; Xiao, 2015). Naked mole-rats represent nature's solution to the problem of both acute and chronic oxygen limitation among mammals, solutions that have developed over evolutionary time. This thesis explored the ability of naked mole-rats to withstand periods of oxygen deprivation or limitation, a trait unique among terrestrial mammals.

For those interested in animal hypoxia tolerance, the naked mole-rat is the natural choice. Naked mole-rats can survive prolonged hypoxia and brief periods of anoxia, and as mammals, they are the most closely related hypoxia-tolerant animal to humans. To date, the physiological and metabolic adaptations displayed by naked mole-rats have mainly been studied in the context of ageing (Buffenstein et al., 2020), neurodegeneration (Orr et al., 2015), pain tolerance (Browe et al., 2020), and cancer resistance (Shepard and Kissil, 2020). More recently, the physiological and molecular mechanisms that allow naked mole-rats to survive hypoxia are being more thoroughly investigated. For example, naked mole-rats were found to depress metabolic rate during hypoxia (Farhat et al., 2020; Pamenter et al., 2019a) and tightly regulate gene transcripts, proteins, posttranslational modifications (Al-attar et al., 2020; Hawkins et al., 2019; Xiao et al., 2017a), signalling pathways (Xiao et al., 2017b), and protein chaperones (Nguyen et al., 2019). Each of 
these studies has revealed new information about the mechanisms contributing to the hypoxia tolerance of this animal.

This thesis aimed to better situate naked mole-rats as a model for hypoxia tolerance and to investigate four main questions regarding the regulatory molecular mechanisms that protect and facilitate this extreme metabolic adaptation. Firstly, I profiled the state of hypoxia-induced oxidative damage and activation of neutralization and repair mechanisms across naked mole-rat tissues, to determine the degree of oxidative damage experienced by naked mole-rats (Chapter 2). Secondly, I identified the neuronal pro-survival mechanisms in place to mitigate hypoxia-induced damage in naked mole-rats, and to determine if brains are susceptible to these damages (Chapter 3). Thirdly, I used next-generation sequencing to examine miRNA expression and their effect on whole brain transcriptional regulation in response to hypoxia (Chapter 4). Lastly, I identified a miRNA-mediated AMPK-driven mechanism for metabolic reorganization that supports tissuespecific prioritization of energy use by more essential organs during hypoxia (Chapter 5).

\subsection{Minimal oxidative damage and enhanced protection during hypoxia}

In Chapter 2, I was able to take a 'snapshot' of the oxidative damage state that naked molerats experience during hypoxia. Profiling the levels of irreversibly oxidized macromolecules during both acute and chronic hypoxia exposures in six tissues (brain, heart, liver, skeletal muscle, kidney, and white adipose tissue) revealed that levels of DNA and RNA oxidation were minimal and that no changes were observed in protein carbonylation during hypoxia. By contrast, lipid peroxidation was elevated during hypoxia in all tissues, with liver displaying an approximately 9.5 -fold increase. This was investigated along with total antioxidant capacity and levels of DNA damage repair 
proteins, all of which were found to exhibit tissue-specific differential expression, with heart and brain exhibiting substantially enhanced antioxidant capacity during hypoxia.

One of the main conclusions that was drawn from this study was that naked mole-rats experience minimal hypoxia-induced oxidative damage, possibly due to their high tolerance of oxidative stress or to their use of hypometabolism to mitigate the potential extent of oxidative stress by reducing energy requirements when oxygen supply is limited. Additionally, this study determined that naked mole-rat brain appears to be relatively resilient to hypoxia-induced oxidative damage compared with other mammals. The enhanced antioxidant capacity displayed by naked mole-rats, along with the limited levels of oxidative damage markers found in brain, set the stage for Chapters 3 and 4, where I examined protective, specifically neuroprotective mechanisms, in place to facilitate successful hypoxia tolerance.

In Chapter 3 I focused on well-known cell-stress related proteins to determine if they are responsible for modulating the brain's resiliency to oxidative damage. Hypoxic brains exhibited a HIF1 $\alpha$-centered response that involved the induction of NFKB, carbonic anhydrase 9, and p21. Collectively, these proteins contributed to maintaining neural integrity by facilitating cell-cycle arrest, anti-apoptosis, and energy conservation. Neuroprotection was also characterized in my nontargeted global miRNA-sequencing analysis performed in Chapter 4 that demonstrated a microRNA-mediated mechanism for eliciting neuroprotective defenses. It interesting to note that some of predicted miRNA targets identified, such as HIF1 $\alpha$ and NFKB, were corroborated in Chapter 3, providing a miRNA mechanism for induction of these targets. 


\subsection{MicroRNA drives fuel conservation and reprioritization during hypoxia}

The differential miRNA expression reported in this thesis provides a mechanism for (1) metabolic depression via the suppression of energy expensive processes, and (2) switching from a lipid-based catabolism to carbohydrate-based metabolism. Both were observed in brain (Chapter 4) and skeletal muscle (Chapter 5).

Active regulation of anaerobic metabolism has been characterized in hypoxia-tolerant brains and we know that naked mole-rat brains suppress mitochondrial oxidation during hypoxia when they switch from a mixed carbohydrate/lipid energy profile to a strictly carbohydrate-centered state (Boutilier, 2001; Pamenter et al., 2019a). However, the molecular mechanisms driving this shift are not well-characterized. In Chapter 4 I report the first small RNA sequencing performed on naked mole-rats. In that study, I identified hypoxia-triggered miRNA regulation that supports a shift away from mitochondria-centered fatty acid oxidation and towards glycolytic and fructose metabolism, mediated by differentially regulated miRNAs. These miRNAs were predicted to both stimulate glycolysis while also dampen key steps in the pathway, possibly to coordinate an overall decrease in energy expenditure and a reliance on carbohydrates when oxygen is limited. These findings are in contrast with previous work that showed that during anoxia, naked mole-rats activated a fructose-driven anaerobic glycolytic pathway for energy production. My findings demonstrate that hypoxic brains behave metabolically different from anoxic brains in naked molerats.

A look at Chapter 5 also shows miRNA metabolic regulation during hypoxia, this time in skeletal muscle. In this study we reported an approximate $80 \%$ decrease in metabolic rate, and a targeted RT-qPCR approach identified hypoxia-induced differential miRNAs that were implicated in AMPK downregulation. Immunoblotting revealed the downregulation of activated 
phosphorylated AMPK, GS, and GLUT4 along with unchanging levels of ACC1 during hypoxia. Collectively, these changes can reduce AMPK activity, favour carbohydrate catabolism, and suppress metabolism overall. This possibly supports tissue-specific prioritization of energy for more essential organs during hypoxia.

\subsection{Future directions}

My thesis has broadened our understanding of naked mole-rat hypoxia tolerance, but as with most studies, they tend to generate more questions than answers. My experiments are no exception. As such possible future directions emerge from the limitations of my studies and the information found within. Firstly, profiling oxidative damage markers and neutralization and repair mechanisms in Chapter 2 could be expanded to also include multi-tissue examinations of antioxidant enzyme activities, along with measurements of individual antioxidants. While a few antioxidant enzymes have previously been examined in hypoxic naked mole-rats, these studies focused on the regulation of antioxidant systems in ageing only (Braude et al., 2021; Munro et al., 2019). It would also be interesting to investigate the state of oxidative damage after allowing animals a brief reoxygenation period post-hypoxic exposure. Indeed, while recovery states were not examined in this thesis, these findings could prove insightful.

Possible expansions to Chapter 3 would be to replicate cell-stress protein profiling in other tissues, as brains are not the only organ that must engage protective mechanisms to overcome the challenge of hypoxia-induced hypometabolism. Additionally, the hypoxia-inducible transcription factors, proteins, and enzymes that were found to be implicated in hypoxic brains should be followed up to examine downstream functional outcomes. 
The miRNA-seq analysis conducted in Chapter 4 identified pathways that were predicted to be regulated in response to hypoxia. The complexity of miRNA regulation sets the stage for various types of follow-up experiments that can dissect the potential of these miRNAs. It was not possible to perform downstream investigations of all the predicted miRNA targeted pathways and processes identified in Chapter 4. Therefore, future work can explore these pathways to determine their importance in facilitating naked mole-rat hypoxia tolerance.

Global miRNA sequencing analysis was only performed on brains, whereas the targeted RTqPCR miRNA experiment was conducted on skeletal muscle. The brain is a complex tissue made up of multiple regions and numerous cell types. Examining miRNA expression in specific brain regions, rather than in whole brains, using microdissection tools would help us to better characterize miRNA responses. Studies have also highlighted the functional importance of organelle-specific expression of miRNAs, an example of which are mitochondria-enriched miRNAs (Macgregor-Das and Das, 2018). This can be explored by isolating organelles, extracting organelle-specific RNA populations from each, and then measuring levels of miRNAs of interest using qPCR or next generation sequencing tools. This can be further expanded to examine other tissues, e.g. analysis of heart and liver would be valuable. A look at circulating miRNAs identities and levels in blood and exosomes could also help to identify biomarkers for hypoxia tolerance. This would add to the recent examination of naked mole-rat extracellular vesicles that reported post-translation modifications of key immune and metabolic proteins, as well as the regulation of three key microRNAs involved in hypoxia and inflammation (Pamenter et al., 2019b).

Another interesting avenue to explore would be to mine the small RNA transcriptome to identify and characterize novel naked mole-rat specific miRNAs. Machine learning predictions tools such as miRDeep can be used to evaluate all RNA sequences for the typical miRNA 
characteristics (An et al., 2013; Chen et al., 2019). Predicted miRNAs can be biologically verified using RT-qPCR and downstream targets can be both predicted in silico and experimentally verified using luciferase promoter assays (Jin et al., 2013).

MicroRNA post-transcriptional regulation works by storing mRNA transcripts in subcellular granules or by permanently degrading them, and in hypometabolic states, transcript storage allows animals to safeguard transcripts until translation resumes. Investigating the fate of miRNA-targeted transcripts during hypoxia would help us further delineate the outcomes of the miRNA regulation reported in Chapters 4 and 5. Indeed, hibernating squirrels, another hypometabolic mammal, were found to form large numbers of stress granules and/or P-bodies containing translationally-stalled mRNA transcripts (Tessier et al., 2014). Furthermore, of the non-coding RNAs that have been shown to regulate cellular processes, this thesis only examined the involvement of miRNAs. Future work should also look at long non-coding RNAs and circular RNAs, both of which have been shown to regulate miRNAs themselves, as well as overall gene expression (Wang et al., 2016).

Herein, AMPK was mainly examined for its role in energy usage, however, it is also involved in regulating autophagy and cell growth, via ULK1, FOXO3A, and mTOR (Alers et al., 2012; Chiacchiera and Simone, 2010). Therefore, it would also be instructive to investigate these pathways and processes, especially because we have shown that AMPK is differentially regulated in naked mole-rate muscles during hypoxia. In addition, it would be interesting to replicate the AMPK immunoblotting study in naked mole-rat brain. This will allow us to determine if the AMPK mechanism identified in skeletal muscles is also responsible for energy reprioritization in brains and other naked mole-rat organs. 
Due to the limited biochemical and molecular work that has been performed on naked molerats, there are many questions that require future investigation. A great place to start would be to exploit the non-bias power of 'big data' to understand the global regulatory crosstalk that is underpinning hypoxia tolerance adaptations. This can be done by generating and analyzing transcriptomic, proteomic, phospho-proteomic, metabolomic, as well as lipidomic datasets. However, such a big experiment will be resource expensive and will require experienced bioinformaticians to integrate the findings and interpret them in the appropriate biological context.

A final area that has yet to be explored in hypoxic naked mole-rats is epigenetics. While information in the genome gives instructions as to what is expressed, epigenetic mechanisms such as DNA methylation and histone modification dictate when genes are expressed (Zhang et al., 2020). DNA methylation typically acts to repress gene transcription as it sterically hinders the binding of transcriptional activators (Cheng and Blumenthal, 2008; Watt and Molloy, 1988). Histone modifications can influence chromatin packing and render genes relaxed and transcriptionally permissive or condensed and transcriptionally repressive (Bannister and Kouzarides, 2011). Exploring how these mechanisms are regulated during hypoxia would provide a global view of which genes are getting turned on when. 


\subsection{Final conclusions}

My thesis builds on previous studies that investigated the many unique adaptations that naked mole-rats have evolved to survive their subterranean lifestyles. The data collected in this thesis paints a picture of intricate signalling mechanisms in place to facilitate metabolic reorganization and protection during hypoxia. In Chapter 2 I determined that naked mole-rats are not as vulnerable to hypoxia-induced oxidative damage, as compared to hypoxia intolerant animals, and that brains appear to be the most resilient. The cell-survival proteins I profiled in Chapter $\mathbf{3}$ identified the induction of mechanisms responsible for conserving energy and maintaining neural integrity under low oxygen levels. In Chapter 4 I performed the first microRNA-seq analysis in naked mole-rats, focusing on the hypoxic brain. Hypoxia-induced miRNAs suppressed ATPexpensive processes, activated central signalling pathways, and coordinated a shift to non-fructose based anaerobic glycolysis. Metabolic reorganization was further examined in Chapter 5, where I provide evidence for a mechanism that may support tissue-specific prioritization of energy for more essential organs in severe hypoxia. This chapter reports an $\sim 80 \%$ decrease in metabolic rate along with a miRNA-mediated, AMPK-driven shift to carbohydrate metabolism in hypoxic skeletal muscles. Taken together, these findings advance our understanding of mammalian hypoxia tolerance and highlight the molecular mechanisms and complex layered regulatory controls required to endure frequent hypoxia exposures imposed by a subterranean lifestyle, as well as provide directions for future studies that should be pursued. 


\subsection{References}

Al-attar, R., Childers, C.L., Nguyen, V.C., Pamenter, M.E., Storey, K.B., 2020. Differential protein phosphorylation is responsible for hypoxia-induced regulation of the Akt/mTOR pathway in naked mole-rats. Comp. Biochem. Physiol. Part A Mol. Integr. Physiol. 242, 110653.

Alers, S., Loffler, A.S., Wesselborg, S., Stork, B., 2012. Role of AMPK-mTOR-Ulk1/2 in the Regulation of Autophagy: Cross Talk, Shortcuts, and Feedbacks. Mol. Cell. Biol. 32, 2-11.

An, J., Lai, J., Lehman, M.L., Nelson, C.C., 2013. MiRDeep*: An integrated application tool for miRNA identification from RNA sequencing data. Nucleic Acids Res. 41, 727-737.

Bannister, A.J., Kouzarides, T., 2011. Regulation of chromatin by histone modifications. Cell Res.

Boutilier, R.G., 2001. Mechanisms of cell survival in hypoxia and hypothermia. J. Exp. Biol. 204, 3171-3181.

Braude, S., Holtze, S., Begall, S., Brenmoehl, J., Burda, H., Dammann, P., del Marmol, D., Gorshkova, E., Henning, Y., Hoeflich, A., Höhn, A., Jung, T., Hamo, D., Sahm, A., Shebzukhov, Y., Šumbera, R., Miwa, S., Vyssokikh, M.Y., von Zglinicki, T., Averina, O., Hildebrandt, T.B., 2021. Surprisingly long survival of premature conclusions about naked mole-rat biology. Biol. Rev. 96, 376-393.

Browe, B.M., Vice, E.N., Park, T.J., 2020. Naked Mole-Rats: Blind, Naked, and Feeling No Pain. Anat. Rec. 303, 77-88.

Buffenstein, R., Lewis, K.N., Gibney, P.A., Narayan, V., Grimes, K.M., Smith, M., Lin, T.D., Brown-Borg, H.M., 2020. Probing pedomorphy and prolonged lifespan in naked mole-rats and dwarf mice. Physiology.

Chen, L., Heikkinen, L., Wang, C., Yang, Y., Sun, H., Wong, G., 2019. Trends in the development of miRNA bioinformatics tools. Brief. Bioinform.

Cheng, X., Blumenthal, R.M., 2008. Mammalian DNA Methyltransferases: A Structural Perspective. Structure.

Chiacchiera, F., Simone, C., 2010. Cell Cycle The AMPK-FoxO3A axis as a target for cancer treatment 1091-1096.

Fago, A., Jensen, F.B., 2015. Hypoxia tolerance, nitric oxide, and nitrite: Lessons from extreme animals. Physiology.

Farhat, E., Devereaux, M.E.M., Pamenter, M.E., Weber, J.M., 2020. Naked mole-rats suppress energy metabolism and modulate membrane cholesterol in chronic hypoxia. Am. J. Physiol. - Regul. Integr. Comp. Physiol. 319, R148-R155.

Hawkins, L.J., Hadj-Moussa, H., Nguyen, V.C., Pamenter, M.E., Storey, K.B., 2019. Naked molerats activate neuroprotective proteins during hypoxia. J. Exp. Zool. Part A Ecol. Integr. 
Physiol. 331, 571-576.

Jin, Y., Chen, Z., Liu, X., Zhou, X., 2013. Evaluating the microRNA targeting sites by luciferase reporter gene assay. Methods Mol. Biol. 936, 117-27.

Krivoruchko, A., Storey, K.B., 2015. Turtle anoxia tolerance: Biochemistry and gene regulation. Biochim. Biophys. Acta - Gen. Subj.

Macgregor-Das, A.M., Das, S., 2018. A microRNA's journey to the center of the mitochondria. Am. J. Physiol. - Hear. Circ. Physiol.

Munro, D., Baldy, C., Pamenter, M.E., Treberg, J.R., 2019. The exceptional longevity of the naked mole-rat may be explained by mitochondrial antioxidant defenses. Aging Cell 18, e12916.

Nguyen, V.C., Deck, C.A., Pamenter, M.E., 2019. Naked mole-rats reduce the expression of ATPdependent but not ATP-independent heat shock proteins in acute hypoxia. J. Exp. Biol. 222.

Orr, M.E., Garbarino, V.R., Salinas, A., Buffenstein, R., 2015. Sustained high levels of neuroprotective, high molecular weight, phosphorylated tau in the longest-lived rodent. Neurobiol. Aging 36, 1496-1504.

Pamenter, M.E., Dzal, Y.A., Thompson, W.A., Milsom, W.K., 2019a. Do naked mole-rats accumulate a metabolic acidosis or an oxygen debt in severe hypoxia? J. Exp. Biol. 222.

Pamenter, M.E., Uysal-Onganer, P., Huynh, K.W., Kraev, I., Lange, S., 2019b. Post-translational deimination of immunological and metabolic protein markers in plasma and extracellular vesicles of naked mole-rat (Heterocephalus glaber). Int. J. Mol. Sci. 20.

Shepard, A., Kissil, J.L., 2020. The use of non-traditional models in the study of cancer resistance - the case of the naked mole-rat. Oncogene.

Storey, K.B., 2004. Adventures in oxygen metabolism. Comp. Biochem. Physiol. - B Biochem. Mol. Biol.

Tessier, S.N., Audas, T.E., Wu, C.-W., Lee, S., Storey, K.B., 2014. The involvement of mRNA processing factors TIA-1, TIAR, and PABP-1 during mammalian hibernation. Cell Stress Chaperones 19, 813-25.

Wang, Y., Hou, J., He, D., Sun, M., Zhang, P., Yu, Y., Chen, Y., 2016. The emerging function and mechanism of ceRNAs in cancer. Trends Genet. 32, 211-224.

Watt, F., Molloy, P.L., 1988. Cytosine methylation prevents binding to DNA of a HeLa cell transcription factor required for optimal expression of the adenovirus major late promoter. Genes Dev. 2, 1136-1143.

Xiao, B., Li, L., Xu, C., Zhao, S., Lin, L., Cheng, J., Yang, W., Cong, W., Kan, G., Cui, S., 2017 a. Transcriptome sequencing of the naked mole-rat (Heterocephalus glaber) and identification of hypoxia tolerance genes. Biol. Open 6, 1904-1912. 
Xiao, B., Wang, S., Yang, G., Sun, X., Zhao, S., Lin, L., Cheng, J., Yang, W., Cong, W., Sun, W., Kan, G., Cui, S., 2017b. HIF-1 $\alpha$ contributes to hypoxia adaptation of the naked mole-rat. Oncotarget 8, 109941-109951.

Xiao, W., 2015. The hypoxia signaling pathway and hypoxic adaptation in fishes. Sci. China Life Sci.

Zhang, L., Lu, Q., Chang, C., 2020. Epigenetics in Health and Disease, in: Advances in Experimental Medicine and Biology. Springer, pp. 3-55. 
Appendices 


\section{Appendix A: List of publications}

\section{PUBLISHED MANUSCRIPTS [26]}

Total of 26 published studies and review articles.

\# denotes equal contribution

\section{Review articles: [5]}

26) Hadj-Moussa H, Hawkins LJ, \& Storey KB. Role of MicroRNAs in Extreme Animal Survival Strategies. MiRNomics II: MicroRNA Biology and Computational Analysis. Springer Science. Book Chapter. In Press.

25) Hadj-Moussa H, Storey KB. (2020). The OxymiR response to oxygen limitation: a comparative microRNA perspective. Journal of Experimental Biology. 18;223(Pt 10): jeb204594. REVIEW.

24) Hadj-Moussa H, Storey KB. (2019). Bringing nature back: using hibernation to reboot organ preservation. The FEBS journal. 286(6), 1094-1100. REVIEW.

23) Hadj-Moussa H, Green GR, and Storey KB. (2018). The living dead: Mitochondria and metabolic arrest. IUBMB Life. 70(12), 1260-1266. REVIEW.

22) Zhang J, Hadj-Moussa H, \& Storey KB. (2016). Current progress of high-throughput microRNA differential expression analysis and random forest gene selection for model and non-model systems: An R implementation. Journal of Integrative Bioinformatics. 13, 5: 306. REVIEW.

\section{Journal articles: [21]}

21) Hadj-Moussa H, Chiasson S, Cheng H, Eaton L, Storey KB, \& Pamenter ME. (2021) MicroRNA-mediated inhibition of AMPK coordinates tissue-specific downregulation of skeletal muscle metabolism in hypoxic naked mole-rats. Journal of Experimental Biology. 1;224(15):jeb242968.

20) Taylor D, Germano J, Song Y, Hadj-Moussa H, Marek-Iannucci S, Dhanji R, Sin J, Czer LSC, Storey KB, Gottlieb RA. (2021). Hypothermia promotes mitochondrial elongation in cardiac cells via inhibition of drp1. Cryobiology. 29:S0011-2240(21)00137-1.

19) Gupta A\#, Hadj-Moussa H\#, Al-attar R, Seibel BA, \& Storey KB. (2021). Hypoxic jumbo squid activate neuronal apoptosis but not MAPK or antioxidant enzymes during oxidative stress. Physiological and Biochemical Zoology. May-Jun 2021;94(3):171-179. 
18) Breedon SA, Hadj-Moussa H, and Storey KB. (2021). Nrf2 activates antioxidant enzymes in the anoxia-tolerant red-eared slider turtle, Trachemys scripta elegans. Journal of Experimental Zoology Part A: Ecological and Integrative Physiology.

17) Hadj-Moussa H, Pamenter ME, and Storey KB. (2021). Hypoxic naked mole-rat brains use microRNA to coordinate hypometabolic fuels and neuroprotective defenses. Journal of Cellular Physiology.

16) Hadj-Moussa H, Wade SC, Childers CL, Storey KB. (2021). Mind the GAP: Purification and characterization of a urea resistant glyceraldehyde-3-phosphate (GAPDH) in extreme dehydration. Proteins: Structure, Function, and Bioinformatics. May;89(5):544-557.

15) Zhang J, Hadj-Moussa H, Storey KB. (2020). Marine periwinkle stress-responsive microRNAs: A potential factor to reflect anoxia and freezing survival adaptations. Genomics. 112(6), 4385-4398.

14) Hadj-Moussa H, Wijenayake S, Storey KB. (2020). Multi-tissue profile of NFkB pathway regulation during mammalian hibernation. Comparative Biochemistry and Physiology Part B: Biochemistry and Molecular Biology. 246, 110460.

13) Hadj-Moussa H, Zhang J, Pifferi, F, Perret, M, Storey KB. (2020). MicroRNAs mediate metabolic rate depression in primate skeletal muscles during torpor. Biochimica et Biophysica Acta (BBA)-Gene Regulatory Mechanisms. 1863(1), 194473.

12) Hawkins LJ, Hadj-Moussa H, Nguyen, VC, Pamenter ME, \& Storey KB. (2019). Naked molerats activate neuroprotective proteins during hypoxia. Journal of Experimental Zoology Part A: Ecological and Integrative Physiology.

11) Hadj-Moussa H, Watts AJ, \& Storey KB. (2019). Genes of the undead: hibernation and death display different gene profiles. FEBS letters. 593(5), 527-532.

10) Hoyeck MP, Hadj-Moussa H, \& Storey KB. (2019). Estivation-responsive microRNAs in a hypometabolic terrestrial snail. PeerJ. 7, e6515.

9) English SG, Hadj-Moussa H, \& Storey KB. (2018). MicroRNAs regulate survival in oxygendeprived environments. Journal of Experimental Biology, 221(23), jeb190579.

8) Hadj-Moussa H, Logan SM, Seibel BA, \& Storey KB. (2018). Potential role for microRNA in regulating hypoxia-induced metabolic suppression in jumbo squids. Biochimica et Biophysica Acta (BBA)-Gene Regulatory Mechanisms, 1861(6), 5856-593.

7) Hadj-Moussa H, \& Storey KB. (2018). Micromanaging freeze tolerance: the biogenesis and regulation of neuroprotective microRNAs in frozen brains. Cellular and Molecular Life Sciences, 1-13.

6) Hoyeck MP, Hadj-Moussa H, \& Storey KB. (2017). The role of MEF2 transcription factors in dehydration and anoxia survival in Rana sylvatica skeletal muscle. PeerJ, 5, e4014. 
5) Aguilar OA, Hadj-Moussa H, \& Storey KB. (2017). Freeze-responsive regulation of MEF2 proteins and downstream gene networks in muscles of the wood frog, Rana sylvatica. Journal of thermal biology, 67, 1-8.

4) Hadj-Moussa H, Moggridge JA, Luu BE, Quintero-Galvis JF, Gaitán-Espitia JD, Nespolo RF, \& Storey KB. (2016). The hibernating South American marsupial, Dromiciops gliroides, displays torpor-sensitive microRNA expression patterns. Scientific Reports. 6: 24627.

3) Aguilar OA, Hadj-Moussa H, \& Storey KB. (2016). Regulation of SMAD transcription factors during freezing in the freeze tolerant wood frog, Rana sylvatica. Comparative Biochemistry and Physiology Part B: Biochemistry and Molecular Biology. 201: 64-71.

2) Liu J, Hagberg I, Novitsky L, Hadj-Moussa H, \& Avis TJ. (2014). Interaction of antimicrobial cyclic lipopeptides from Bacillus subtilis influences their effect on spore germination and membrane permeability in fungal plant pathogens. Fungal Biology. 118(2): 855-861.

1) Katzenback BA, Holden HA, Falardeau J, Childers CL, Hadj-Moussa H, Avis TJ, \& Storey KB. (2014). Regulation of the Rana sylvatica brevinin-1SY antimicrobial peptide during development and in dorsal and ventral skin in response to freezing, anoxia, and dehydration. Journal of Experimental Biology. 217(8): 1392-1401.

\section{MANUSCRIPTS IN PROGRESS [9]}

\section{Manuscripts under review: [1]}

9) Williamson SM, Ingelson-Filpula WA, Hadj-Moussa H, \& Storey KB. Epigenetic underpinnings of freeze avoidance in the goldenrod gall moth, Epiblema scudderiana. Journal of Insect Physiology.

\section{Manuscripts in preparation: [8]}

8) Hadj-Moussa H, Pamenter ME, and Storey KB. Naked mole-rats resist the accumulation of hypoxia-induced oxidative damage.

7) Hadj-Moussa H, Lamontagne J, \& Storey KB. MicroRNAs facilitate anoxic survival in turtle brains.

6) Wade SC, Hadj-Moussa H, \& Storey KB. RNA m6A methylation in frozen and anoxic vertebrate brains.

5) Hawkins LJ, Hadj-Moussa H, \& Storey KB. Naturally freeze tolerant oocytes rely on cryoprotectant glycerol accumulation driven by microRNA expression. 
4) Hadj-Moussa H, \& Storey KB. Biogenesis and regulation of the freeze-thaw responsive microRNA fingerprint in wood frog liver.

3) Kyle T, Hadj-Moussa H, \& Storey KB. Multi-stress profiling of miRNA biogenesis and expression in wood frog kidneys.

2) Rehman S, Hawkins LJ, Hadj-Moussa H, \& Storey KB. Regulation of wood frog FOXO protein expression during freezing.

1) Hadj-Moussa H, Makia Y, Storey KB. Regulation of histone methylation in hypoxic Jumbo squid. 


\section{Appendix B: Communications at scientific meetings}

\section{Conferences: [25]}

* denotes presenting author.

25) Hadj-Moussa $H^{*}$ and Storey KB. The Answer to All of Your Questions is: MicroRNA. Institute of Engineering and Medicine BioSciTech Symposium, University of Minnesota. Apr 12, 2021. Oral Presentation.

24) Ingelson-Filpula $\mathrm{AW}^{*}$, Hadj-Moussa H, Storey KB. The Biogenesis and MicroRNA Transcriptomics of Freeze-Tolerant Grey Tree Frogs (Hyla versicolor). Apr 6, 2021. Poster Presentation.

23) Hadj-Moussa $\mathbf{H}^{*}$. MicroRNAs: Can we use natural gene silencers to solve human problems? Carleton University Virtual Science Café. Feb 10, 2021. Oral Presentation. Link to recorded presentation.

22) Storey KB* \& Hadj-Moussa H. Metabolic arrest: Deep Space. The Science of Suspended Animation in Deep Space. Virtual workshop. Translational Research Institute for Space Health (TRISH). Aug 7, 2020. Oral Presentation.

21) Hadj-Moussa $\mathbf{H}^{*}$, \& Storey KB. MicroRNAs facilitate metabolic rate depression in torpid primates: That's cool, now what? 10th International Congress of Comparative Physiology and Biochemistry (ICCPB2019). Ottawa, Canada. Aug 5-9, 2019. Oral presentation.

20) Hadj-Moussa H*, Watts AJ, \& Storey KB. Genes of the undead: hibernation and death display different gene profiles. 10th International Congress of Comparative Physiology and Biochemistry (ICCPB2019). Ottawa, Canada. Aug 5-9, 2019. Oral presentation.

19) Taylor D*, Germano J, Marek-Iannucci S, Hadj-Moussa H, Storey K, Gottlieb R. Hypothermic regulation of cardiac mitochondrial dynamics. 56th Annual Meeting of the Society for Cryobiology (CRYO2019), San Diego, California, United States. July 21-25, 2019. Oral Presentation.

18) Hadj-Moussa $\mathbf{H}^{*}$, \& Storey KB. Micromanaging freeze tolerance: The biogenesis and regulation of cryoprotective microRNAs in frozen brains and livers. Toronto RNA Enthusiasts Day 2019 (TREnD2019). SickKids Peter Gilgan Centre for Research and Learning, Toronto, Ontario, Canada. July 30, 2019. Oral presentation. *Winner of best talk.

17) Hadj-Moussa $H^{*}$, Watts AJ, \& Storey KB. Genes of the undead: Hibernation and death display different gene profiles. 16th Annual Ottawa-Carleton Institute of Biology Graduate Student Symposium (OCIB2019), Carleton Univ., May. 2, 2019. Oral presentation.

16) Hadj-Moussa H*, Watts AJ, \& Storey KB. Genes of the undead: Hibernation and death display different gene profiles. 21st Annual Chemistry \& Biochemistry Graduate Research Conference (CBGRC2018), Concordia Univ., Nov. 9, 2018. Oral presentation. 
15) Hadj-Moussa H, Moggridge JA, Luu BE, Quintero-Galvis JF, Gaitán-Espitia JD, Nespolo $\mathrm{RF}$, \& Storey KB*. The hibernating South American marsupial, Dromiciops gliroides, displays torpor-sensitive microRNA expression patterns. Fourth International congress of the Serbian society for mitochondrial and free radical physiology. University of Belgrade, Serbia. September 28-30, 2018. Poster. International conference.

14) English SG, Hadj-Moussa H, \& Storey KB*. MicroRNAs regulate survival in oxygendeprived environments. 13th annual meeting of the International Conference on Genomics (ICG-13). Oct. 24-28, 2018 in Shenzhen, China. Poster. International conference.

13) Hoyeck MP, Hadj-Moussa H, \& Storey KB*. Regulation of MEF2 proteins and downstream targets in muscles of dehydrates and anoxic wood frogs. 13th Annual meeting of the International Conference on Genomics (ICG-13). Oct. 24-28, 2018 in Shenzhen, China. Poster. International conference.

12) Hadj-Moussa $\mathbf{H}^{*}, \&$ Storey KB. The biogenesis and regulation of microRNAs in a natural model of vertebrate freeze tolerance. Toronto RNA enthusiasts Day (TReND). August 2, 2017. Provincial conference. Poster.

11) Hadj-Moussa $\mathbf{H}^{*}$, \& Storey KB. The biogenesis and regulation of microRNAs in a natural model of vertebrate freeze tolerance. Canadian Society of Molecular Biosciences. Ottawa, ON, May 16-20, 2017. Poster. National conference.

10) Hadj-Moussa H, Moggridge JA, Luu BE, Quintero-Galvis JF, Gaitán-Espitia JD, Nespolo $\mathrm{RF}$, \& Storey KB*. The hibernating South American marsupial, Dromiciops gliroides, displays torpor-sensitive microRNA expression patterns. Canadian Society of Zoologists, Satellite Symposium: 50 years of comparative biochemistry - the legacy of Peter Hochachka. University of Manitoba, Winnipeg, MB, May 13-14, 2017. Poster. National conference.

9) Hadj-Moussa $H^{*}$ \& Storey KB. (2016). Micromanaging freeze tolerance: The role of microRNAs in regulating brain cryoprotection. 53rd Annual Meeting of the Society for Cryobiology (CRYO2016), Ottawa, Canada. Invited Speaker - Oral Presentation.

8) Hadj-Moussa H*, Moggridge JA, Luu BE, Quintero-Galvis JF, Gaitán-Espitia JD, Nespolo RF, \& Storey KB. (2016). The hibernating South American marsupial, Dromiciops gliroides, displays torpor-sensitive microRNA expression patterns. Ottawa-Carleton Institute for Biology Symposium (OCIB2016). May 5-6, 2016. Poster.

7) Hadj-Moussa H*, Moggridge JA, Luu BE, Quintero-Galvis JF, Gaitán-Espitia JD, Nespolo RF, \& Storey KB. (2015). The hibernating South American marsupial, Dromiciops gliroides, displays torpor-sensitive microRNA expression patterns. 18th Annual Chemistry \& Biochemistry Graduate Research Conference (CBGRC2015), Concordia Univ., Nov. 20, 2015. Poster.

6) Hadj-Moussa $\mathbf{H}^{*}$ \& Storey KB. (2015). Regulation of the central metabolic switch, PGC-1 $\alpha$, and interacting transcription factors in frozen and anoxic Rana sylvatica. 28th Annual 
Ontario Biology Day Conference. Poster. * Winner of the Cellular and Molecular Biology Poster Prize.

5) Hadj-Moussa H* \& Storey KB. (2015). Regulation of the central metabolic switch, PGC-1 $\alpha$, and interacting transcription factors in frozen and anoxic Rana sylvatica. Ottawa-Carleton Institute for Biology Symposium (OCIB2015). Poster.

4) Hadj-Moussa H* \& Storey KB. (2015). Regulation of the central metabolic switch, PGC-1 $\alpha$, and interacting transcription factors in frozen and anoxic Rana sylvatica. Carleton University Undergrad Research Day. Poster.

3) Katzenback BA*, Holden HA, Falardeau J, Childers CL, Hadj-Moussa H, Avis TJ, \& Storey KB. (2014). Rana sylvatica brevinin-1SY: regulation of an antimicrobial peptide in response to environmental stress. Montreal, Quebec. Canadian Society of Zoologists (CSZ14), 2014-05-25. Poster.

2) Katzenback BA*, Holden HA, Falardeau J, Childers CL, Hadj-Moussa H, Avis TJ, \& Storey KB. (2013). Regulation of the antimicrobial peptide brevinin-1SY in the skin of Rana sylvatica in response to environmental stress. Cryobiology: International Journal of Low Temperature Biology and Medicine. Society for Cryobiology's 50th Annual Meeting (CRYO2013), 2013-07-28. Poster.

1) Katzenback BA, Holden HA, Falardeau J, Childers CL, Hadj-Moussa H, Avis TJ*, \& Storey KB. (2013). Regulation of the antimicrobial peptide brevinin-1SY in the skin of Rana sylvatica in response to environmental stress. Canadian Society of Microbiologists 63rd Annual Conference, 2013-06-17 (84). Poster.

\section{Conference proceedings: [3]}

3) Taylor D*, Germano J, Marek-Iannucci S, Hadj-Moussa H, Storey K, Gottlieb R. Hypothermic regulation of cardiac mitochondrial dynamics. (2020). Abstract. Cryobiology 91:172.

2) Hadj-Moussa H \& Storey K. (2016). Micromanaging freeze tolerance: The role of microRNAs in regulating brain cryoprotection. Abstract. Cryobiology, 73(3): 427.

1) Katzenback BA, Holden HA, Falardeau J, Childers CL, Hadj-Moussa H, Avis TJ, \& Storey KB. (2013). Regulation of the antimicrobial peptide brevinin-1SY in the skin of Rana sylvatica in response to environmental stress. Abstract. Cryobiology, 67(3): 441. 


\section{Appendix C: Representative western blot images}

Displayed below are full western blot images and their corresponding Coomassie-stained membranes for a representative set of protein targets from Chapter $\mathbf{4}$ and Chapter 5.

A

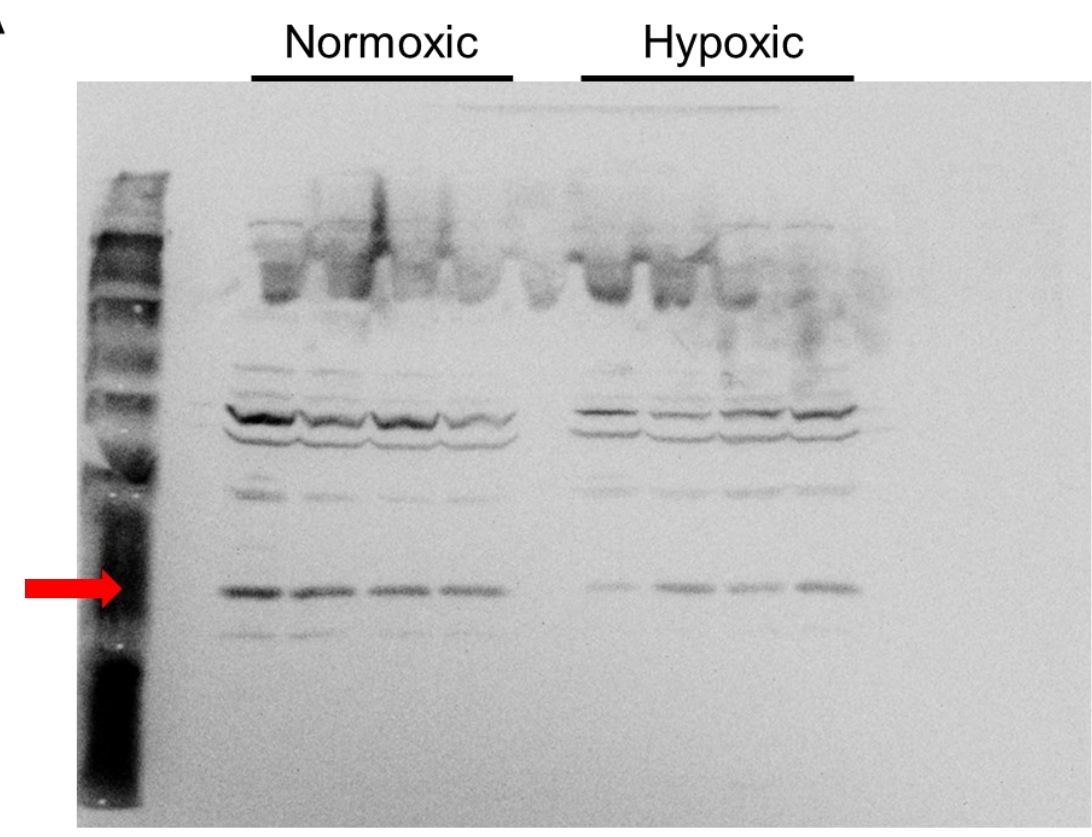

B

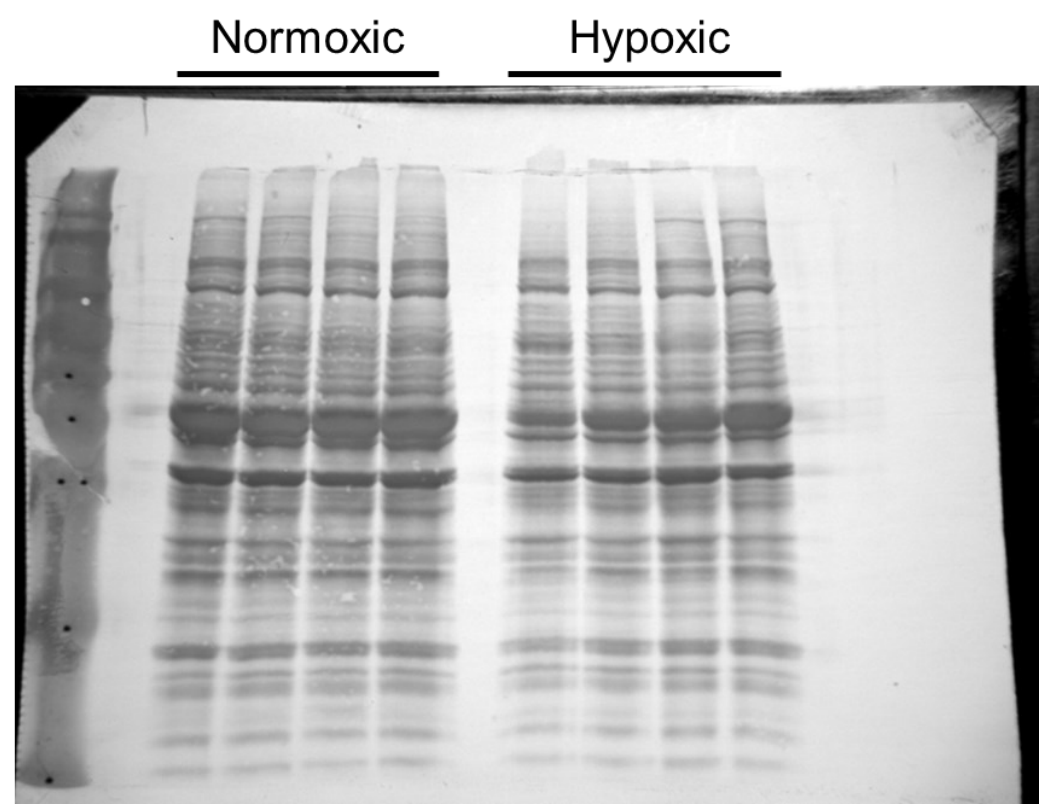

Appendix Figure C1. Full western blot image for ketohexokinase (KHK) in normoxic and hypoxic naked mole-rat brains. [A] Full immunoblot membrane. [B] Full Coomassie-stained membrane. From Chapter 4. 
A

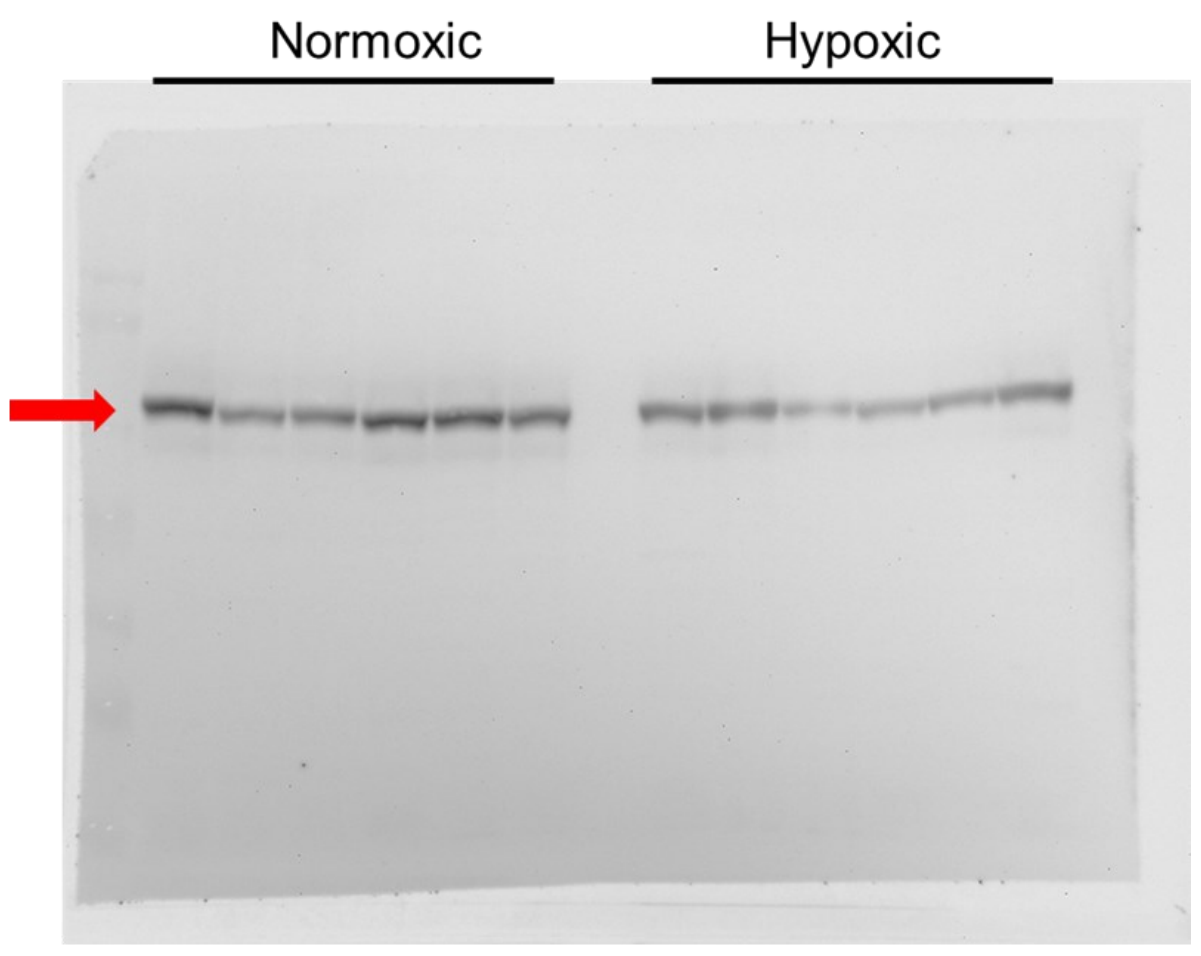

B

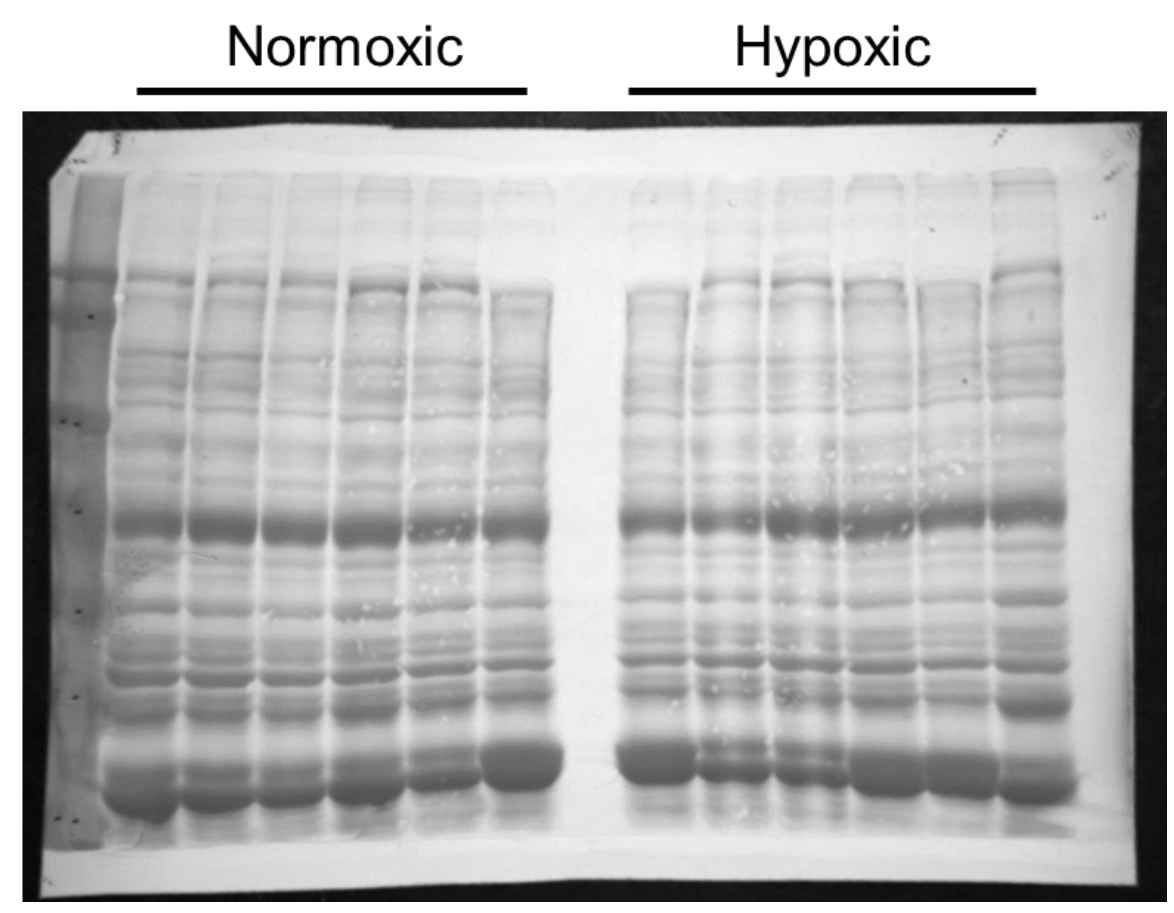

Appendix Figure C2. Full western blot image for phosphorylated glycogen synthase (GS) in normoxic and hypoxic naked mole-rat skeletal muscles. [A] Full immunoblot membrane. [B] Full Coomassiestained membrane. From Chapter 5. 
A
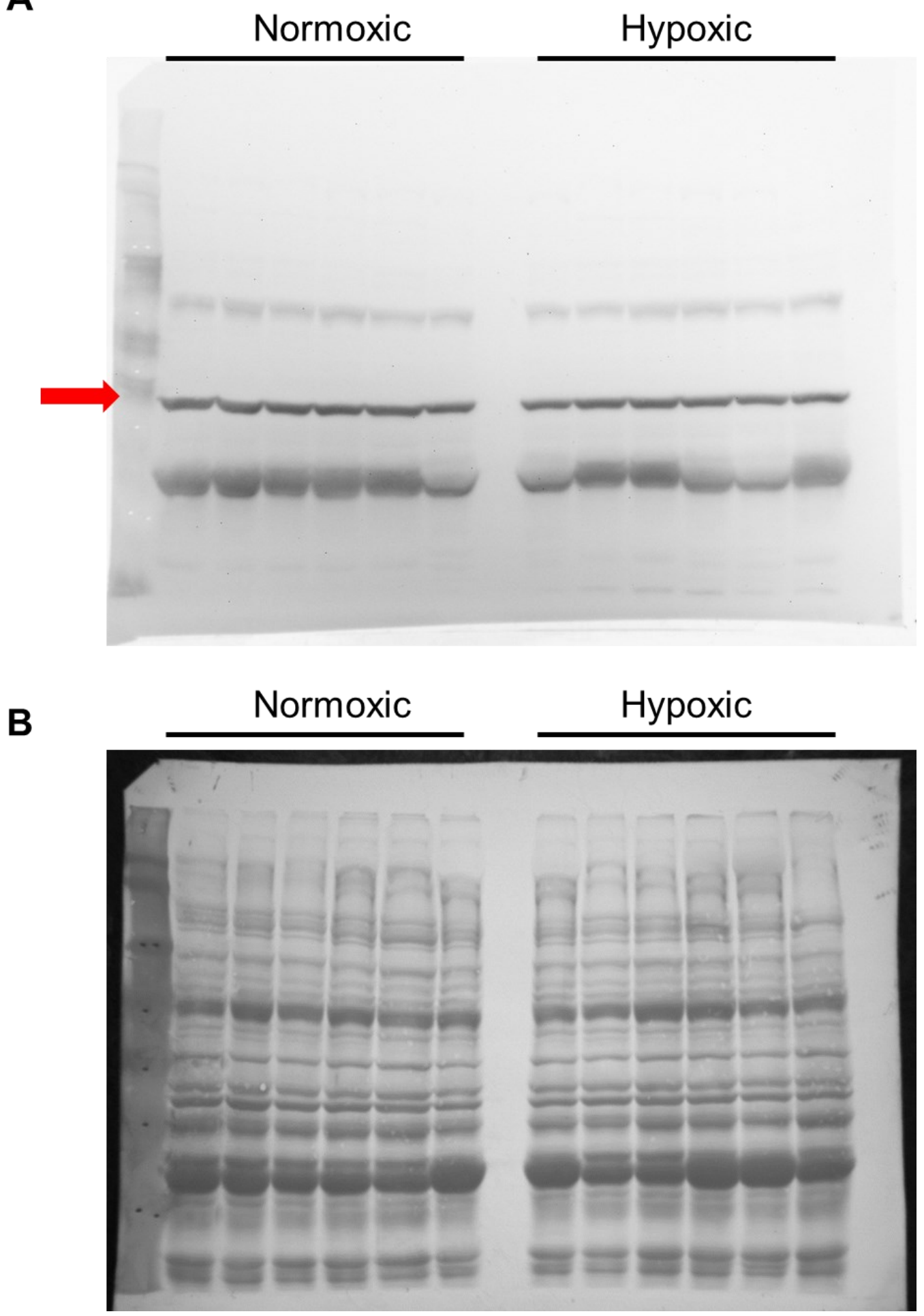

Appendix Figure C3. Full western blot image for glucose transporter 1 (GLUT1) in normoxic and hypoxic naked mole-rat skeletal muscles. [A] Full immunoblot membrane. [B] Full Coomassie-stained membrane. From Chapter 5. 


\section{Appendix D: RT-qPCR quantification}

In this thesis, all microRNA and mRNA RT-qPCR quantifications were performed as follows. Total RNA was extracted from tissues $\left(T_{1}-T_{n}\right)$ from individual animals and cDNA synthesis experiments generated cDNA samples $\left(S_{1}-S_{n}\right)$ (Appendix Figure D1). All cDNA samples were then pooled for each tissue to generate the $S_{\text {pool }}$. This was then used to produce a 2-fold serial dilution standard curve of at least five points $\left(S_{\text {pool }}^{1 / 2}-S_{\text {pool }}^{1 / 32}\right)$ and a no template control $\left(S_{N T C}\right)$.

Next, cDNA samples were diluted to the same dilution corresponding to the middle of the standard curve (1/8 dilution in the case presented in Appendix Figure D1). After amplification, standard curve samples displayed even spacing with an efficiency between $90-110 \%$, with late or no amplification of the NTC sample (Appendix Figure D2A and D2B). When the amplification curves of the diluted samples of interest $\left(S_{l}-S_{n}\right)$ are overlaid, their $C_{q}$ surround their corresponding dilution sample from the standard curve (1/8 in Appendix Figure D2C and D2B). Melt curve analysis showed the amplification of the same single product in all samples (Appendix Figure 2B).

Appendix Figures D1 and D2 are from Hawkins, L.J. (2020). The Molecular Biology of Dehydration Tolerance: Regulation of Gene Expression and Function in Xenopus laevis. $\mathrm{PhD}$ thesis, Carleton University. 


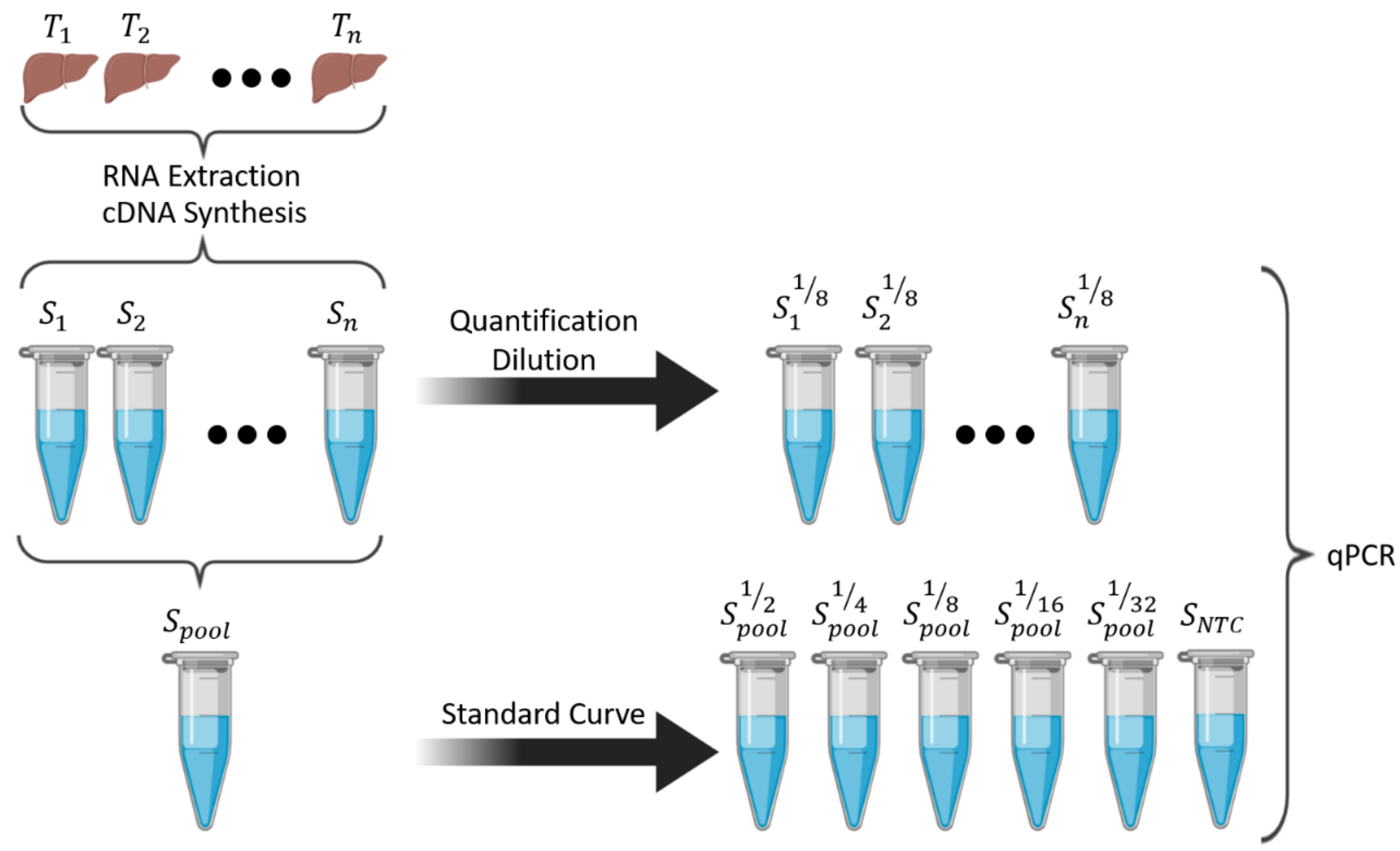

Appendix Figure D1. Sample schema for quantification of mRNAs and microRNAs using RT-qPCR (Hawkins, 2020). 
a

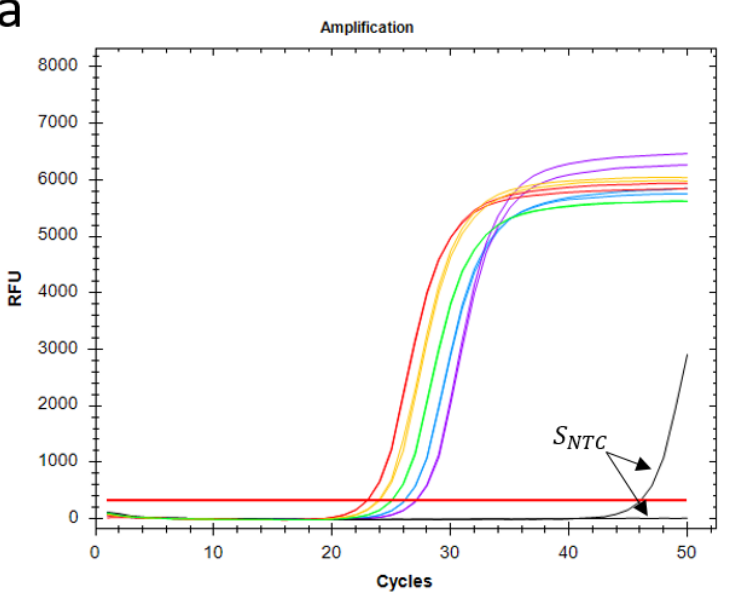

C

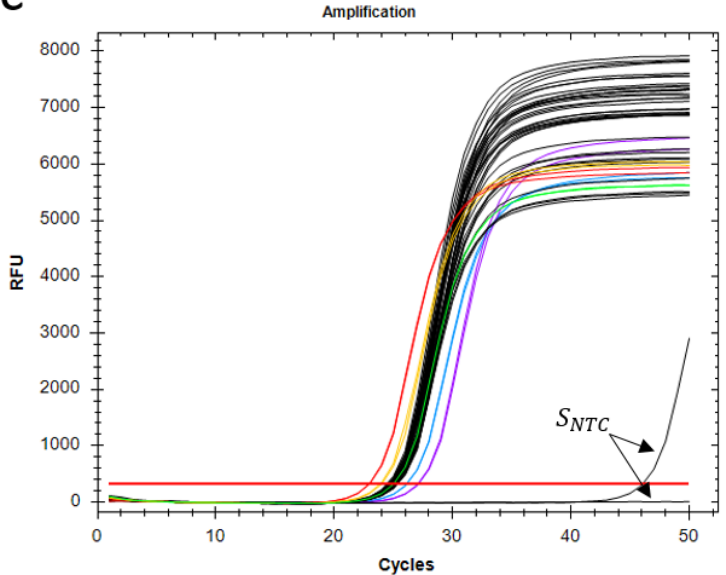

b

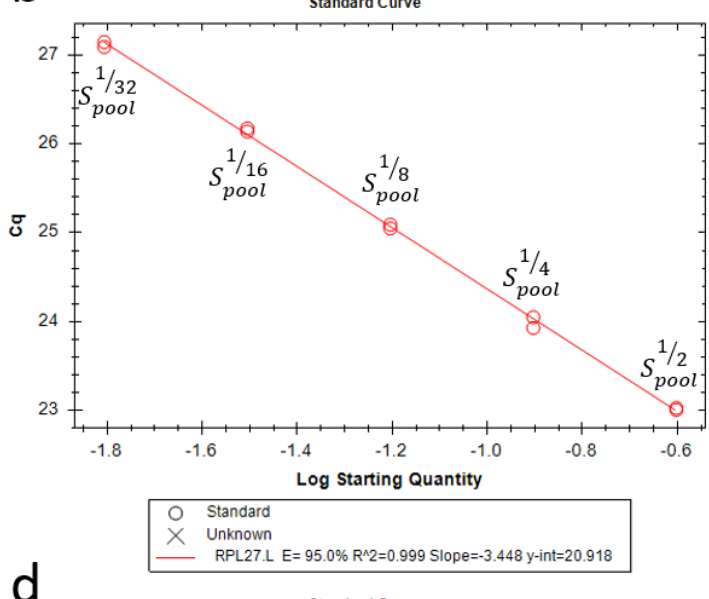

d

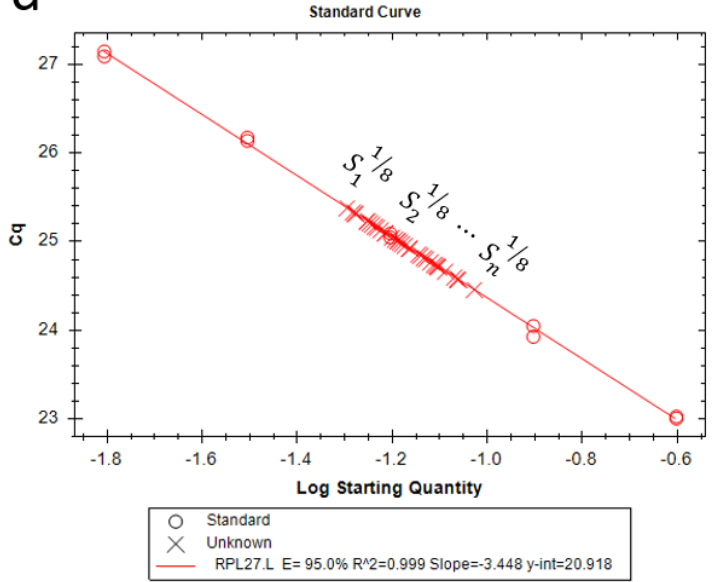

e

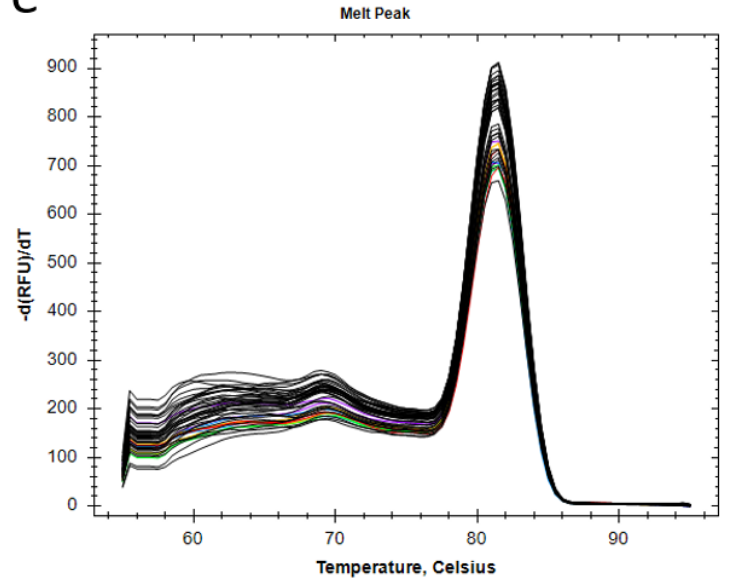

Appendix Figure D2. Example RT-qPCR results. [A] Amplificiation of 2-fold serial dilution standard curve (rainbow curves) shows expected equidistant $C_{q}$ values (red line) and NTC samples amplifying much later than standard curve samples or not at all. [B] Plot of log of the dilution factor versus $C_{q}$ shows reaction efficiency of $95.0 \%$ and $\mathrm{R}^{2}$ of 0.999 . [C] Overlay of amplification of samples of interest. [D] Samples of interest fall near middle of standard curve as expected from dilution. [E] Melt curve analysis indicates a single product was amplified in all samples (Hawkins, 2020). 


\section{Appendix E: RT-qPCR primers used for mRNA and microRNA analysis}

Appendix Table E1. The microRNA and mRNA primers used for RT-qPCR analyses in Chapter 4.

\begin{tabular}{|ll|}
\hline Target & Primer Sequence (5'-3') \\
\hline \hline miRNA & \\
\hline miR-132-3p & ACACTCCAGCTGGGTAACAGTCTACAGCC \\
miR-184-3p & ACACTCCAGCTGGGTGGACGGAGAACTGA \\
miR-383-5p & ACACTCCAGCTGGGAGATCAGAAGGTGAT \\
miR-301a-3p & ACACTCCAGCTGGGCAGTGCAATAGTATT \\
snord66 & ACACTCCAGCTGGGTGCAAACTCGATCA \\
Universal Primer & ACCGGTTCTCGTCCGATCACCGAAGT \\
(reverse) & \\
miRNA Stem-loop & CTCACAGTACGTTGGTATCCTTGTGATGTTCGATGCCATATTGTACTGTGAGT \\
Adapter & TTTTTTTTVN \\
\hline mRNA & \\
\hline GLUT5 (forward) & GGCGTGAACGCGATCTACTA \\
GLUT5 (reverse) & CACGTACTGCACGTCATTGG \\
KHK (forward) & AACAGCACAATGCCAAGCTG \\
KHK (reverse) & ACACCACCTCTCCATAGCCA \\
LDH (forward) & GAAGAATCTGCACCCCGAGT \\
LDH (reverse) & GCCCAGGATGTGTAGCCTTT \\
SREBP2 (forward) & CTGCAAGGTTTTGGACCAC \\
SREBP2 (reverse) & ACCTCTCCATCTCTGCGAGG \\
GAPDH (forward) & TCCCCACAGCTTCTATCCCA \\
GAPDH (reverse) & TGGGTGCAGCAGGCTTTATT \\
\hline
\end{tabular}


Appendix Table E2. The microRNA and mRNA primers used for RT-qPCR analyses in Chapter 5.

\begin{tabular}{|c|c|}
\hline Target & Primer Sequence $\left(5^{\prime}-3^{\prime}\right)$ \\
\hline miR-101a-3p & ACACTCCAGCTGGGTACAGTACTGTGATA \\
\hline $\operatorname{miR}-1224-5 p$ & ACACTCCAGCTGGGGTGAGGACTGGGGAG \\
\hline miR-124-3p & ACACTCCAGCTGGGTAAGGCACGCGGTGA \\
\hline miR-130a-3p & ACACTCCAGCTGGGCAGTGCAATGTTAAA \\
\hline miR-135b-5p & ACACTCCAGCTGGGTATGGCTTTTCATTC \\
\hline miR-137-3p & ACACTCCAGCTGGGTTATTGCTTAAGAAT \\
\hline miR-140-3p & ACACTCCAGCTGGGTACCACAGGGTAGAA \\
\hline miR-140-5p & ACACTCCAGCTGGGCAGTGGTTTTACCCT \\
\hline miR-144-3p & ACACTCCAGCTGGGTACAGTATAGATGAT \\
\hline miR-148b-3p & ACACTCCAGCTGGGTCAGTGCATCACAGA \\
\hline $\operatorname{miR}-15 b-5 p$ & ACACTCCAGCTGGGTAGCAGCACATCATG \\
\hline miR-17-3p & ACACTCCAGCTGGGACTGCAGTGAGGGCA \\
\hline miR-184-3p & ACACTCCAGCTGGGTGGACGGAGAACTGA \\
\hline miR-195a-5p & ACACTCCAGCTGGGTAGCAGCACAGAAAT \\
\hline miR-199a-3p & ACACTCCAGCTGGGACAGTAGTCTGCACA \\
\hline $\operatorname{miR}-199 a-5 p$ & ACACTCCAGCTGGGCCCAGTGTTCAGACT \\
\hline miR-19a-3p & ACACTCCAGCTGGGTGTGCAAATCTATGC \\
\hline $\operatorname{miR}-19 b-3 p$ & ACACTCCAGCTGGGTGTGCAAATCCATGC \\
\hline miR-200c-3p & ACACTCCAGCTGGGTAATACTGCCGGGTA \\
\hline miR-204-3p & ACACTCCAGCTGGGGCTGGGAAGGCAAAG \\
\hline miR-214-3p & ACACTCCAGCTGGGACAGCAGGCACAGAC \\
\hline miR-21a-5p & ACACTCCAGCTGGGTAGCTTATCAGACTG \\
\hline miR-223-3p & ACACTCCAGCTGGGTGTCAGTTTGTCAAA \\
\hline miR-24-3p & ACACTCCAGCTGGGTGGCTCAGTTCAGCA \\
\hline miR-25-5p & ACACTCCAGCTGGGAGGCGGAGACTTGGG \\
\hline miR-26a-5p & ACACTCCAGCTGGGTTCAAGTAATCCAGG \\
\hline miR-27a-3p & ACACTCCAGCTGGGTTCACAGTGGCTAAG \\
\hline miR-290a-5p & ACACTCCAGCTGGGACTCAAACTATGGGG \\
\hline miR-291b-3p & ACACTCCAGCTGGGAAAGTGCATCCATTT \\
\hline miR-296-5p & ACACTCCAGCTGGGAGGGCCCCCCCTCAA \\
\hline miR-29abc-3p & ACACTCCAGCTGGGTAGCACCATCTGAAA \\
\hline miR-300-3p & ACACTCCAGCTGGGTATGCAAGGGCAAGC \\
\hline miR-300-5p & ACACTCCAGCTGGGTTGAAGAGAGGTTAT \\
\hline miR-301a-3p & ACACTCCAGCTGGGCAGTGCAATAGTATT \\
\hline miR-30b-5p & ACACTCCAGCTGGGTGTAAACATCCTACA \\
\hline miR-30e-3p & ACACTCCAGCTGGGCTTTCAGTCGGATGT \\
\hline miR-323-3p & ACACTCCAGCTGGGCACATTACACGGTCG \\
\hline $\operatorname{miR}-325-3 p$ & ACACTCCAGCTGGGTTTATTGAGCACCTC \\
\hline miR-335-5p & ACACTCCAGCTGGGTCAAGAGCAATAACG \\
\hline miR-350-3p & ACACTCCAGCTGGGTTCACAAAGCCCATA \\
\hline miR-351-5p & ACACTCCAGCTGGGTCCCTGAGGAGCCCT \\
\hline
\end{tabular}




\begin{tabular}{|ll|}
\hline Target & Primer Sequence (5' $\mathbf{-} \mathbf{3}^{\prime}$ ) \\
\hline miR-378a-5p & ACACTCCAGCTGGGCTCCTGACTCCAGGT \\
miR-429-3p & ACACTCCAGCTGGGTAATACTGTCTGGTA \\
miR-451b-5p & ACACTCCAGCTGGGTGGGAGCAGCAAGAG \\
miR-455-5p & ACACTCCAGCTGGGTATGTGCCTTTGGAC \\
miR-466d-5p & ACACTCCAGCTGGGTGTGTGTGCGTACAT \\
miR-490-3p & ACACTCCAGCTGGGCAACCTGGAGGACTC \\
miR-503-5p & ACACTCCAGCTGGGTAGCAGCGGGAACAG \\
miR-669e-5p & ACACTCCAGCTGGGTGTCTTGTGTGTGCA \\
miR-691-3p & ACACTCCAGCTGGGATTCCTGAAGAGAGG \\
miR-709-3p & ACACTCCAGCTGGGGGAGGCAGAGGCAGG \\
miR-7a-3p & ACACTCCAGCTGGGCAACAAATCACAGTC \\
miR-875-3p & ACACTCCAGCTGGGCCTGAAAATACTGAG \\
miR-92a-3p & ACACTCCAGCTGGGTATTGCACTTGTCCC \\
miR-let7e-5p & ACACTCCAGCTGGGTGAGGTAGGAGGTTG \\
\hline snord58a & ACACTCCAGCTGGGTCTTAGGACACCTTT \\
snord96a & ACACTCCAGCTGGGATCCTAGTGATGACAAG \\
Universal & \\
Primer & ACCGGTTCTCGTCCGATCACCGAAGT \\
(reverse) & \\
miRNA Stem- & CTCACAGTACGTTGGTATCCTTGTGATGTTCGATGCCATATTGTACTGTGAGTTT \\
loop Adapter & TTTTTVN \\
\hline
\end{tabular}




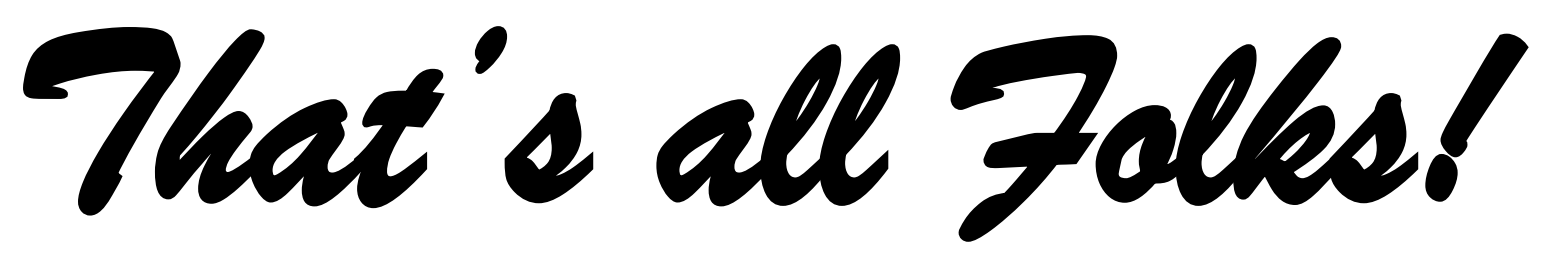

